\title{
Characterization of Cement Paste in Fresh State Using Electrical Resistivity Technique
}

\author{
by
}

Hossein Sallehi

A thesis submitted to the Faculty of Graduate and Postdoctoral Affairs in partial fulfillment of the requirements for the degree of

\section{Master of Applied Science}

in

Civil Engineering

Carleton University

Ottawa, Ontario

Ottawa-Carleton Institute of Civil and Environmental Engineering

(C) 2015, Hossein Sallehi 


\begin{abstract}
The structural and durability performance of concrete depends on its hardened properties such as compressive strength and transport properties. These hardened properties depend on concrete's mixture design and its fresh properties. For a given concrete mixture design, the fresh properties have the most significant influence on hardened and durability properties. In recent years, the electrical resistivity of fresh concrete has gained increasing attention as a performance index because of its practicality and the extent of information it potentially provides on fresh properties. In this research a comprehensive investigation on the relationship between the electrical resistivity of concrete with various mixture design properties was conducted. Impedance spectroscopy technique was used to monitor the electrical resistivity of various cement paste mixtures during first 2 hours after mixing before the setting time. The electrical conductivity and $\mathrm{pH}$ of the pore solutions extracted from fresh cement paste mixtures were also determined. The conductivity of pore solution extracted from some select hardened paste mixtures cured in sealed moisture condition was also obtained. These data were used to investigate the effect of various influential parameters such as (1) the effect of time on the electrical resistivity, (2) the effect of supplementary cementitious materials and chemical admixtures on the electrical resistivity, (3) the effect of w/c ratio on the electrical resistivity, (4) tortuosity and the relationship between electrical resistivity of paste and pore solution and (5) correlation between the $\mathrm{pH}$ and electrical conductivity of pore solution. In addition, a numerical model was proposed to estimate the electrical conductivity of pore solution and its variation with time during the fresh state before setting time based on the properties of mixture design and the chemical composition of the cementitious materials in the mixture.
\end{abstract}




\section{Acknowledgements}

I would like to express my sincere gratitude to my co-supervisors, Dr. Pouria Ghods and Dr. O. Burkan Isgor, for their unlimited advice and guidance throughout this study. Without them, this research would not have been possible. They have been great mentors in every step of my work with their extensive knowledge and experience in this area as well as very friendly and caring supervisors. It has been a great honor for me to work under their supervision. I also am deeply thankful to my nominal supervisor, Dr. Yasser Hassan, for his support during my Master program. He has always been very concerned to help me solving my academic problems.

Words cannot express my deepest appreciation for my family, especially my parents, who have been unwaveringly supporting me since the beginning of my life. They always encouraged me and gave me strength to cope with the difficulties associated with graduate student life. Their unconditional love was always a source of relief especially during hard times. I cannot imagine any of my success without their dedication.

I would like to express my gratitude to Dr. Rahil Khoshnazar for her constructive comments and friendly advice during my entire Master program. Support from the technical team at Giatec Scientific Inc., especially Mustafa Salehi is highly appreciated. Support from National Research Council (NRC) of Canada, especially during the primary steps of my experimental research is highly appreciated.

I am thankful to Dr. Dale P. Bentz from National Institute of Standards and Technology (NIST) for his constructive comments on the numerical modeling of this research. I would like to express my gratitude to the administrative staff of the Department of Civil 
and Environmental Engineering at Carleton University and also to the manager of environmental engineering laboratory, Dr. Marie Jose Tudoret-Chow, for their assistance during my Master program. 


\section{Table of Contents}

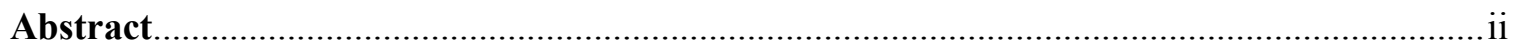

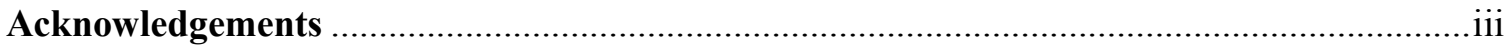

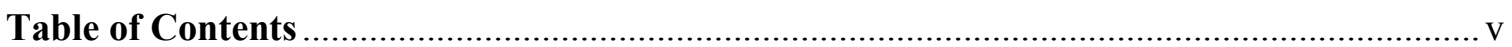

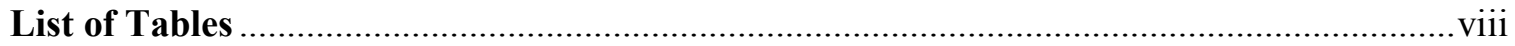

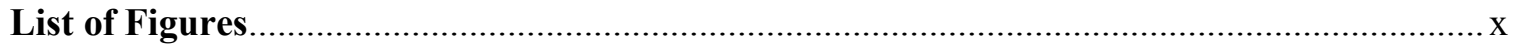

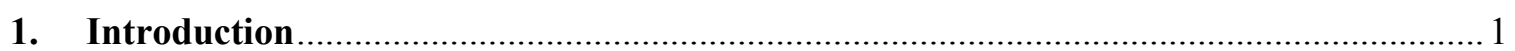

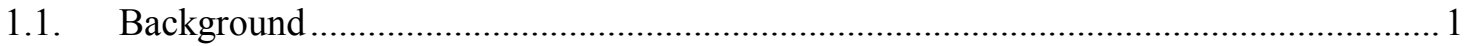

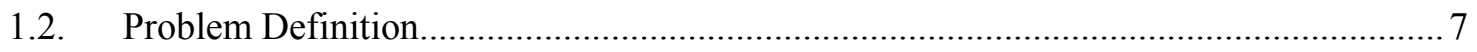

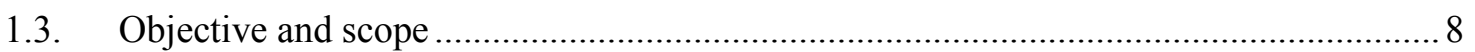

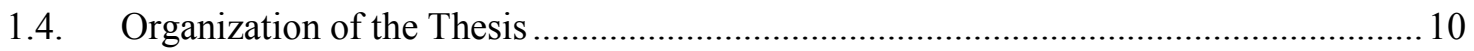

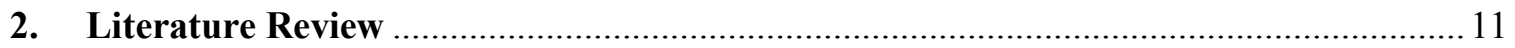

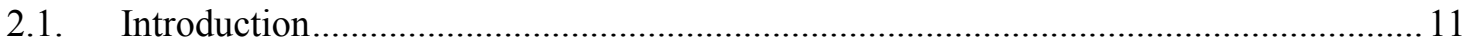

2.2. Application of electrical resistivity for fresh concrete .............................................. 12

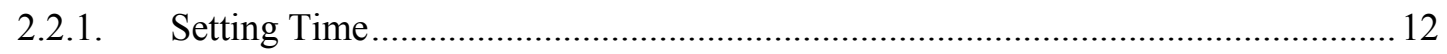

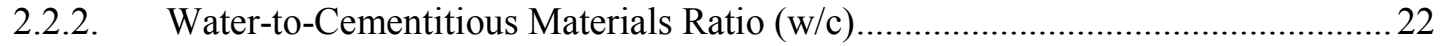

2.3. Electrical resistivity measurement techniques for concrete, mortar and cement paste.. 26

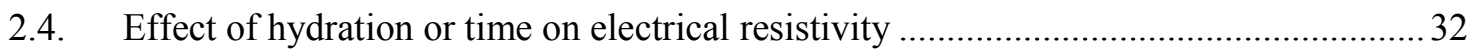

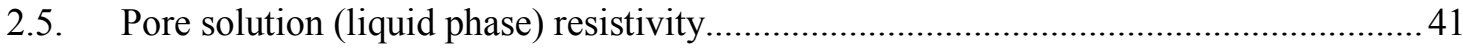

2.6. Temperature effect on electrical resistivity ............................................................ 45

2.7. Effect of supplementary cementitious materials and chemical admixtures on electrical resistivity

2.8. Relationship between bulk electrical resistivity and pore solution resistivity: Archie's Law and Formation Factor F

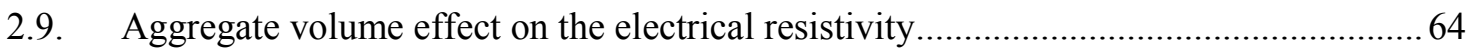

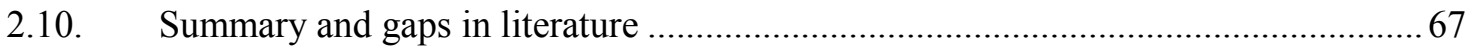

2.10.1. w/c ratio effect on electrical resistivity and applicability of Archie's law in fresh

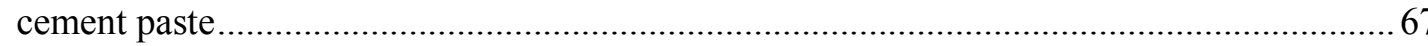

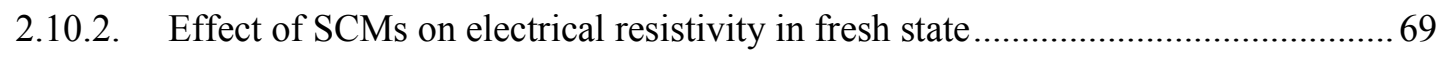


2.10.3. Simple model for the estimation of pore solution conductivity ............................ 70

2.10.4. Conductivity of pore solution versus time: Fresh and hardened state.................... 70

2.10.5. $\mathrm{pH}$ measurement as an alternative to obtain the conductivity of pore solution at

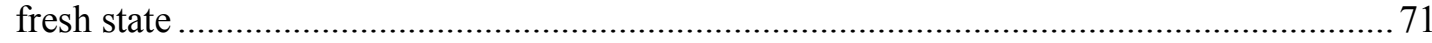

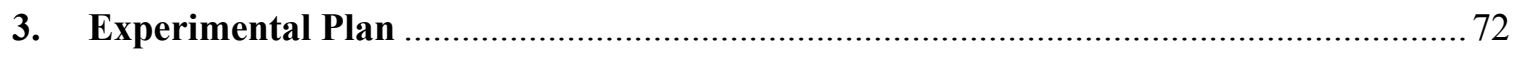

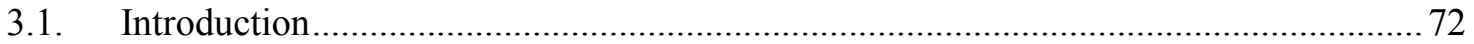

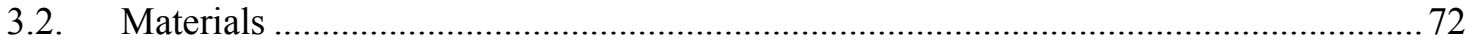

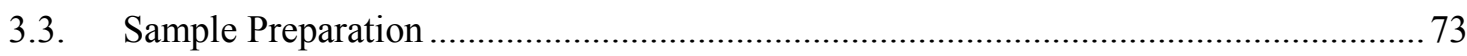

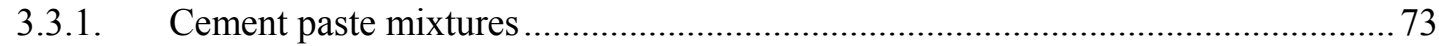

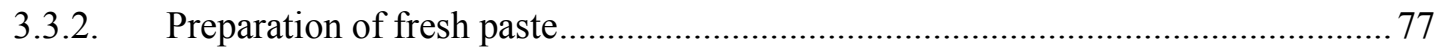

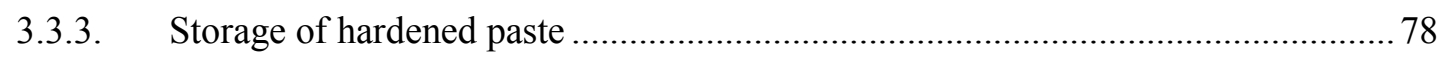

3.3.4. Solution extraction from fresh paste …................................................................ 79

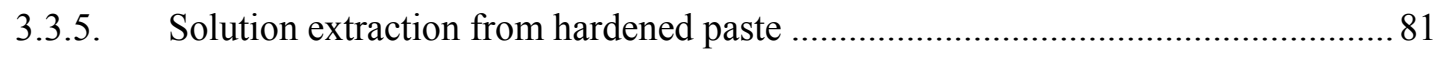

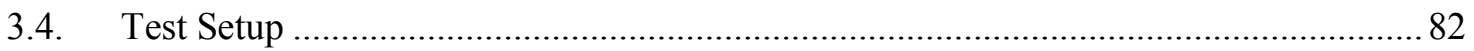

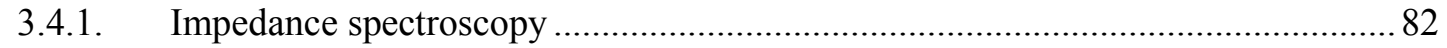

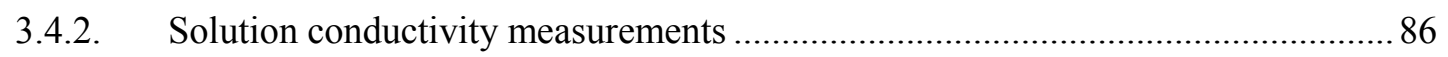

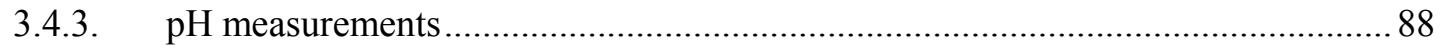

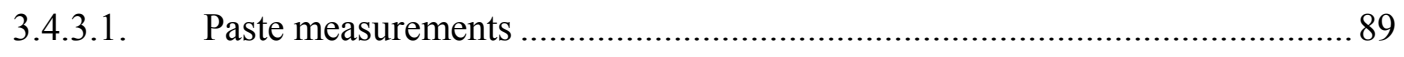

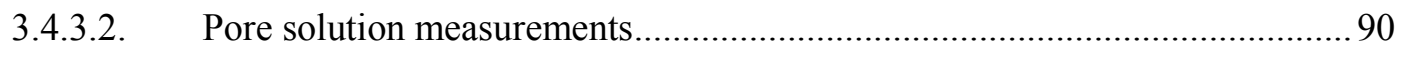

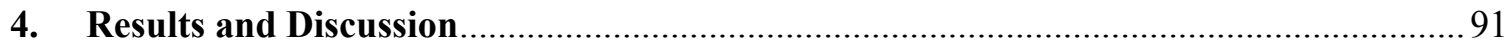

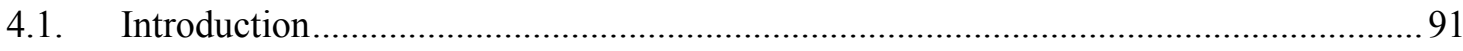

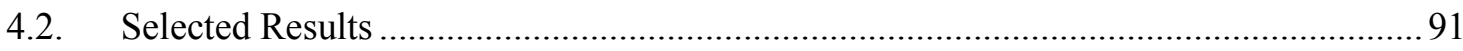

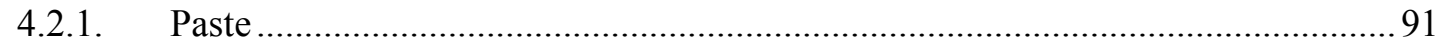

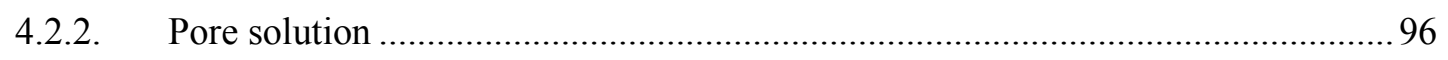

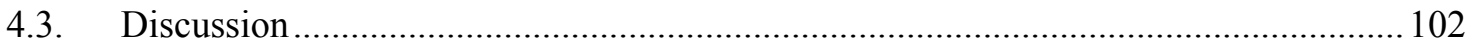

4.3.1. Effect of time on conductivity/resistivity development of fresh paste................ 102

4.3.2. Effect of superplasticizer and SCMs on pore solution conductivity .................... 107

4.3.3. Effect of $\mathrm{w} / \mathrm{c}$ on pore solution conductivity ...................................................... 112 
4.3.4. Relationship between the electrical resistivity of paste and pore solution using Archie's law

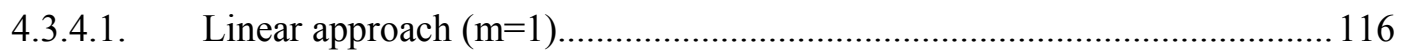

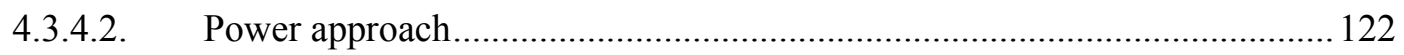

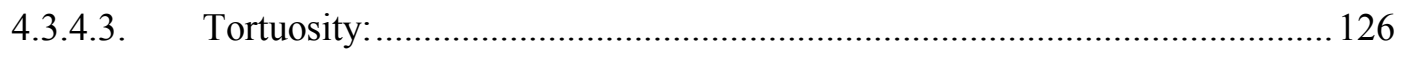

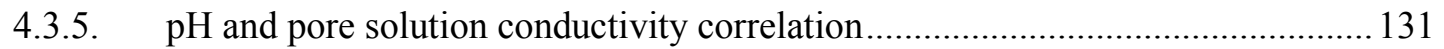

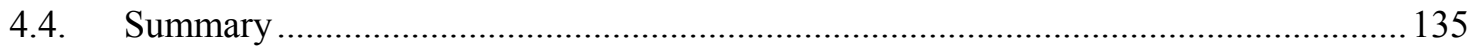

5. Numerical Modeling to estimate the conductivity of pore solution .............................. 138

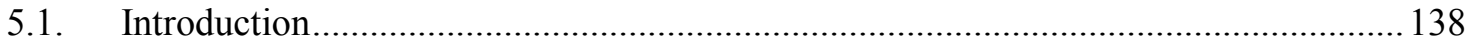

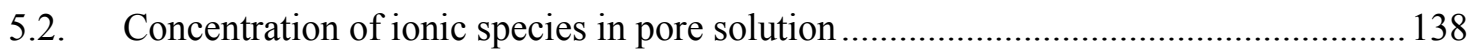

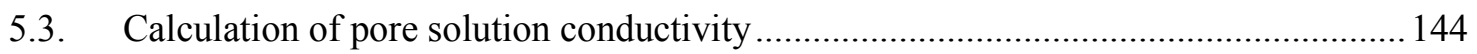

5.4. Proposed model for conductivity of pore solution in fresh state .................................. 146

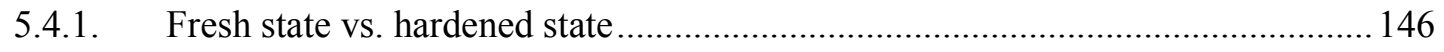

5.4.2. Proposed Model for pore solution conductivity at fresh state.............................. 148

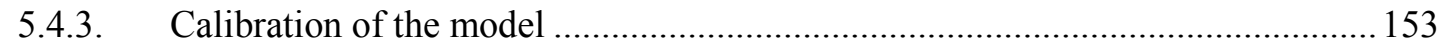

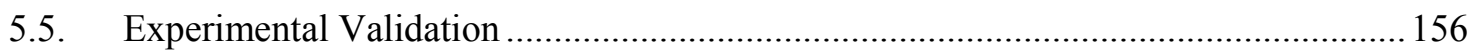

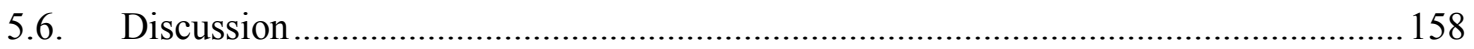

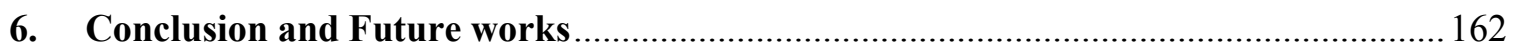

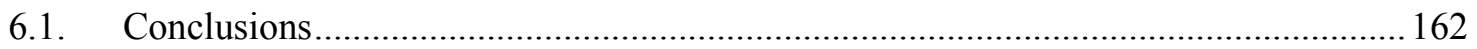

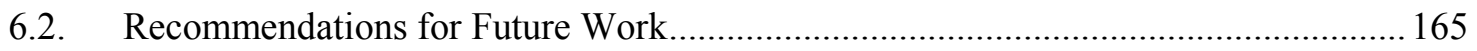

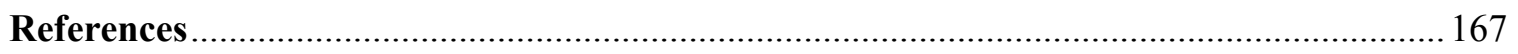

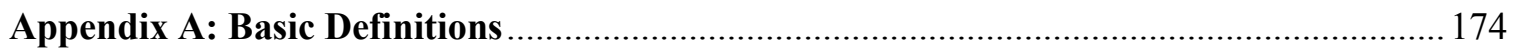

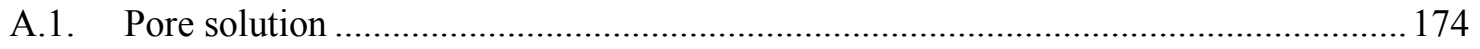

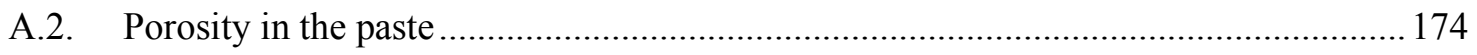

A.3. Chemical and physical effect on paste resistivity .................................................. 175

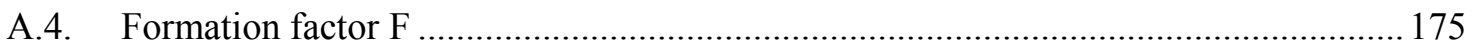

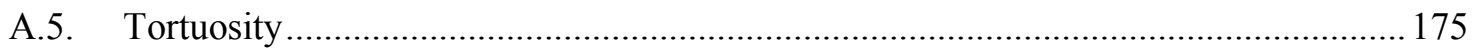

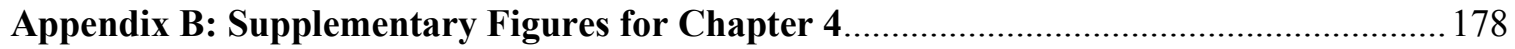




\section{List of Tables}

Table 2.1. Times of occurrence of critical points $t_{m}$ and $t_{t}$ in resistivity response and the setting times of concrete resulted from penetration test [6]. 15

Table 2.2. Physical and chemical properties of OPC and supplementary cementitious materials [13] 19

Table 2.3. Mixture proportions of concrete samples [8]. 26

Table 2.4. Electrical resistivity of concrete samples as a function of time and w/c [8] .. 39

Table 2.5. Electrical resistivity of liquid phase $\rho_{0}\left(\mathrm{t}_{\mathrm{m}}\right)$ at minimum point time of pastes with $w / c$ of $0.3[26]$

Table 2.6. Equations suggested for $E_{a, \rho}$ and $\rho_{21}$ correlation based on the type of concrete mixture [7].

Table 3.1. Chemical and physical properties of cementitious materials 74

Table 3.2. Detailed mixture proportion of the pastes 75

Table 4.1. Paste resistivity and $\mathrm{pH}$ results for OPC plus $30 \%$ fly ash at $30^{\text {th }}$ minute after mixing. 95

Table 4.2. Paste resistivity and $\mathrm{pH}$ results for OPC plus $0.5 \%$ superplasticizer at $30^{\text {th }}$ minute after mixing. 96

Table 4.3. Pore solution conductivity/resistivity and $\mathrm{pH}$ results for OPC plus $30 \%$ fly ash at $30^{\text {th }}$ minute of hydration age.

Table 4.4. Pore solution conductivity/resistivity and $\mathrm{pH}$ results for OPC plus $0.5 \%$ superplasticizer at $30^{\text {th }}$ minute of hydration age. 97

Table 4.5. Average resistivity of fresh paste and pore solution at $30^{\text {th }}, 60^{\text {th }}$ and $90^{\text {th }}$ minute after mixing for paste mixtures with $\mathrm{w} / \mathrm{c}$ of 0.45 . 103 
Table 4.6. Pore solution conductivity of 5 main types of paste mixtures for $30^{\text {th }}, 60^{\text {th }}$ and $90^{\text {th }}$ minute of hydration age at w/c 0.45 . 108

Table 4.7. Particle density of the materials used in the paste mixtures at $25^{\circ} \mathrm{C}$ 115

Table 4.8. Variation of porosity $\varphi$ and connectivity $\beta$ with $w / c$ for ordinary 120

Table 4.9. Inverse of formation factor, $1 / \mathrm{F}$, versus time for pastes with the w/c of 0.45 during the first 2 hours. 121

Table 5.1. Total contents of $\mathrm{Na}_{2} \mathrm{O}$ and $\mathrm{K}_{2} \mathrm{O}$ in four major clinker phases of OPC 141

Table 5.2. Empirical values of constants for OPC hydration at any age in days to be used in Eq. 5.5 [37]. 141

Table 5.3. Modified empirical values of constants for OPC plus fly ash pastes in Eq. $(5.5)$

Table 5.4. Equivalent conductivity at infinite dilution $\lambda^{\circ}$ and conductivity coefficients $G$ at $25^{\circ} \mathrm{C}$ 145

Table 5.5. Total $\mathrm{Na}_{2} \mathrm{O}$ and $\mathrm{K}_{2} \mathrm{O}$ contents in cementitious materials from chemical composition analysis. 153

Table 5.6. Empirical constants obtained from calibration for OPC, fly ash, silica fume and slag in Eq. 5.20. 155 


\section{List of Figures}

Figure 1.1. Relationship between the compressive strength and the electrical resistivity for pastes with different $\mathrm{w} / \mathrm{c}$ ratios and different curing temperatures of 15,20 and $30{ }^{\circ} \mathrm{C}$ [2].

Figure 1.2. Relationship between $w / c$ and electrical resistivity of pastes at early age [14].

Figure 1.3. Conductance-Time curves of OPC plus silica fume paste mixtures. Ia, Ib, Ic and Id represent 10, 20, 30 and 50 percent silica fume replacement, respectively; for w/c of 0.70 at two temperatures: (a) $25^{\circ} \mathrm{C}$; and (b) $45^{\circ} \mathrm{C}$ [9].

Figure 1.4. Pore solution conductivity versus paste specimen age for a paste with $w / \mathrm{c}$ of $0.3[11]$. 5

Figure 1.5. Formation factor of OPC pastes as a function of porosity [5] ........................ 6

Figure 2.1. Electrical resistivity development of concretes to identify: (a) minimum point $\mathrm{P}_{\mathrm{m}}$ on $\rho$-t curves; (b) transition point $\mathrm{P}_{\mathrm{t}}$ on $\rho$-t (log scale) curves [6]. 14

Figure 2.2. Curves of different pastes with w/c of $0.3,0.35$ and 0.4 denoted by P0.3, P0.35 and P0.4; respectively, over 24 hrs: (a) Resistivity development; (b) Rate of resistivity development [14].

Figure 2.3. Resistivity development versus time for paste with different w/c ratios; i.e., P0.3, P0.4 and P0.5 are paste samples with w/c of $0.3,0.4$ and 0.5 , respectively; while P0.4- $\mathrm{KCl}$ is a paste incorporated with $1 \% \mathrm{KCl}$ by mass in cementitous materials: a) during first 1440 minutes; b) during first 200 minutes [5]. 18

Figure 2.4. Correlation of setting time $\left(t_{\text {ini }}, t_{\text {fin }}\right)$ resulted from penetration test and inflection time ratio $K_{t}[25]$. (Note: Squares and diamonds show final and initial setting times, respectively). 
Figure 2.5. Electrical resistivity development of pastes with the w/c of 0.3 and containing $0 \%, 0.1 \%, 0.15 \%$ and $0.2 \%$ retarder denoted by L0.0, L0.1, L0.15 and L0.2, respectively [26]. 21

Figure 2.6. Schematic representation of cement paste structure with time $t$ and corresponding hydration degree $\alpha[5]$. 23

Figure 2.7. Electrical resistivity versus time of hydration for two concrete samples with aggregate volume fraction of $60 \%$ [27]. 24

Figure 2.8. Electrical resistivity versus w/c of concrete: a) No fly ash; b) $25 \%$ fly ash [8].

Figure 2.9. Block diagram of the electrolytic ohmmeter [31]. 27

Figure 2.10. Electrolytic ohmmeter mechanism: A) Applied current; B) Corresponding voltage across concrete [31] 29

Figure 2.11. Schematic of non-contact resistivity measurement of cement paste [5] ..... 29

Figure 2.12. Non-contact electrical resistivity measurement instrument and mold cross section [15] 30

Figure 2.13. Electrical resistivity probe: a) actual probe; b) schematic shape [8]........... 32 Figure 2.14. Bulk electrical resistivity and resistivity rate of fresh concrete with the w/c of 0.40: (a) Electrical resistivity development $\rho(\mathrm{t})-\mathrm{t}$; (b) Rate of resistivity development $\mathrm{d} \rho(\mathrm{t}) / \mathrm{dt}-\mathrm{t}[15]$. 37

Figure 2.15. Degree of hydration with time during first $48 \mathrm{hr}$ for concrete samples [15]. (Solid curves are calculated from Eq. 2.12, whereas the points show the experimental results).

Figure 2.16. Conductance-Time curves of OPC plus silica fume paste mixtures. Ia, Ib, Ic and Id represent 10,20, 30 and 50 percent silica fume replacement, respectively; for w/c of 0.55 at two temperatures: (a) $25^{\circ} \mathrm{C}$; and (b) $45^{\circ} \mathrm{C}$ [9]. 40 
Figure 2.17. Electrical resistivity of pore solution $\left(\rho_{0}\right)$ in cement paste samples with various $\mathrm{w} / \mathrm{c}$ ratios [14]. 45

Figure 2.18. Resistivity variation with temperature for a concrete sample with w/c of 0.4 (labelled by $41 \mathrm{~A})$ in saturated and unsaturated ( $85 \%$ and $92 \%$ relative humidity) curing conditions after 6 years [7]. 48

Figure 2.19. Calculated percentage change in resistivity per ${ }^{\circ} \mathrm{C}$ using: a) Eq. 2.22; b) Eq. $2.23[7]$. 51

Figure 2.20. Electrical resistivity development and inflection point $\left(t_{i}\right)$ identification for control paste $\left(\mathrm{P}_{0}\right)$ with: a) $0.8 \%$ of SP1; and b) $0.25 \%$ of SP2 [25]. 56

Figure 2.21. The average of normalized resistivity $(\overline{N R})$ as a function of aggregate volume fraction $\left(\mathrm{V}_{\mathrm{a}}\right)$ for concrete samples [27]. 63

Figure 2.22. Electrical resistivity development with time during 24 hours for concrete samples with different aggregate volume fraction $\mathrm{V}_{\mathrm{a}}$ : a) $\mathrm{w} / \mathrm{c}=0.4, \mathrm{~b}$ ) $\mathrm{w} / \mathrm{c}=0.5$ [27]. .. 66

Figure 3.1. Fresh paste preparation: a) water addition to cementitious materials; b) homogenous paste after mixing. 78

Figure 3.2. Collection of hardened paste samples in a sealed curing condition. 79

Figure 3.3. Solution extraction process from fresh paste: a) test setup to apply suction through vacuum pump; b) sample collection 80

Figure 3.4. Schematic shape of pore press (source: Benoit Fournier) ........................... 82

Figure 3.5. Section of test setup for electrical resistivity measurement. 85

Figure 3.6. Paste sample resistivity measurement: a) electrodes anchored with spacer to keep them in parallel position; b) test setup for electrical resistivity measurement with

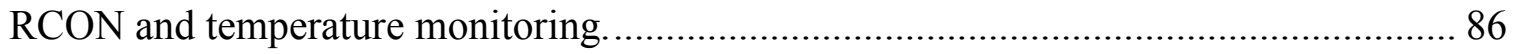

Figure 3.7. Conductivity measurement test for pore solution 87 
Figure 3.8. $\mathrm{pH}$ measurement test for: a) paste; b) pore solution 89

Figure 4.1. Impedance-frequency spectra in one cycle of frequency sweep. Although measurements were taken at different times, for clarity, data from only two sweeps (at 30 minutes and 2 hours) are shown. 92

Figure 4.2. Phase angle-frequency spectra in one cycle of frequency sweep. Although measurements were taken at different times, for clarity, data from only two sweeps (at 30 minutes and 2 hours) are shown 93

Figure 4.3. Paste and pore solution resistivity variations with $\mathrm{w} / \mathrm{c}$ and their ratio for OPC plus $30 \%$ fly ash at $30^{\text {th }} \mathrm{min}$..... 98

Figure 4.4. Paste and pore solution resistivity variations with $\mathrm{w} / \mathrm{c}$ and their ratio for OPC plus $0.5 \%$ superplasticizer at $30^{\text {th }} \mathrm{min}$. 98

Figure 4.5. Resistivity results from reproducibility tests and their average as well as error bars with 1 standard deviation from average: a) OPC plus $0.5 \%$ superplasticizer; b) OPC plus $10 \%$ silica fume; c) OPC plus $30 \%$ fly ash. 100

Figure 4.6. Electrical resistivity development with time during fresh state and error bars with one standard deviation from average for the paste mixtures with w/c of 0.45: a) pore solution; b) paste. 104

Fig. 4.7. Pore solution conductivity of selected paste mixtures at fresh state compared to that of hardened state at around 5 months old. 107

Figure 4.8. Pore solution conductivity ( $\sigma$ pore solution) development with time for five general types of paste mixtures including OPC, OPC plus $0.5 \%$ superplasticizer, OPC plus $30 \%$ fly ash, OPC plus $10 \%$ silica fume and OPC plus $30 \%$ slag at w/c $0.45 \ldots . . .109$

Figure 4.9. Pore solution conductivity versus $\mathrm{w} / \mathrm{c}$ ratio for paste samples at $30^{\text {th }}$ minute of hydration age. 113

Figure 4.10. Paste and pore solution resistivity as well as inverse of formation factor versus w/c at $30^{\text {th }}$ minute of paste age: a) Ordinary Portland Cement; b) OPC plus $0.5 \%$ 
superplasticizer; c) OPC plus 30\% fly ash; d) OPC plus $10 \%$ silica fume; and e) OPC plus $30 \%$ slag.

Figure 4.11. Inverse of formation factor, $1 / \mathrm{F}$, versus time for pastes with the $\mathrm{w} / \mathrm{c}$ of 0.45 during the first 2 hours.

Figure 4.12. Formation factor versus porosity of fresh cement pastes at $30^{\text {th }}$ minute of paste age: a) Ordinary Portland Cement (OPC); b) OPC plus 0.5\% superplasticizer; c) OPC plus 30\% fly ash; d) OPC plus 10\% silica fume; and e) OPC plus 30\% slag....... 123

Figure 4.13. Formation factor versus porosity of fresh cement paste at $30^{\text {th }}$ minute of paste age considering all different paste mixtures. 125

Figure 4.14. Regression analysis to calculate the tortuosity of ordinary portland cement paste when exponent $\mathrm{m}$ is equal to 1.99 . 126

Figure 4.15. Tortuosity values of paste mixtures at fresh state. 127

Figure 4.16. Formation factor versus $\mathrm{w} / \mathrm{c}$ ratio of fresh cement paste at $30^{\text {th }}$ minute of paste age considering all different paste mixtures 130

Figure 4.17. $\mathrm{pH}$ of the pore solution versus $\mathrm{w} / \mathrm{c}$ of the pastes at $30^{\text {th }}$ minute after mixing. 131

Figure 4.18. $\mathrm{pH}$ development in pore solution with time for the pastes having w/c of 0.45 . 132

Figure 4.19. Pore solution conductivity and $\mathrm{pH}$ correlation at fresh cement paste. 133

Figure 4.20. Paste and pore solution $\mathrm{pH}$ relationship in cement paste. 134

Figure 5.1. Calculated and experimental conductivity relationship after calibration of the proposed model for $\mathrm{w} / \mathrm{c}$ of 0.45 . 155

Figure 5.2. Calculated pore solution conductivity from the proposed model versus those from experimental data. 157 
Figure 5.3. Calculated pore solution conductivity from the proposed model versus those from the experimental data. 158

Figure 5.4. Pore solution conductivity development over time calculated by the proposed model for paste mixtures with w/c of 0.45 during first 2 hours. 161

Figure A.1. Schematic representation of cement paste structure in fresh state. 174

Figure A.2. Schematic of the ions transport in the pore solution among the solid particles with the same porosity: a) normal distribution of particles; b) aggregated particles; c) round shape particles; and d) small size particles.

Figure B.1. Paste and pore solution resistivity as well as inverse of formation factor versus w/c at $30^{\text {th }}$ minute of paste age: a) OPC plus $0.2 \%$ superplasticizer; b) OPC plus 1.0\% superplasticizer; c) OPC plus 10\% fly ash; d) OPC plus 50\% fly ash; e) OPC plus $5 \%$ silica fume; f) OPC plus 15\% silica fume; g) OPC plus $10 \%$ slag; h) OPC plus 50\% slag 178

Figure B.2. Formation factor versus porosity of fresh cement pastes at $30^{\text {th }}$ minute of paste age: a) OPC plus $0.2 \%$ superplasticizer; b) OPC plus $1.0 \%$ superplasticizer; c) OPC plus $10 \%$ fly ash; d) OPC plus 50\% fly ash; e) OPC plus 5\% silica fume; f) OPC plus $15 \%$ silica fume; g) OPC plus 10\% slag; h) OPC plus 50\% slag 180

Figure B.3. Formation factor versus w/c ratio of fresh cement pastes at 30th minute of paste age: a) OPC; b) OPC plus $0.2 \%$ superplasticizer; c) OPC plus $0.5 \%$ superplasticizer; d) OPC plus 1.0\% superplasticizer ; e) OPC plus 10\% fly ash; f) OPC plus $30 \%$ fly ash ; g) OPC plus 50\% fly ash; h) OPC plus 5\% silica fume; i) OPC plus 10\% silica fume ; j) OPC plus 15\% silica fume; k) OPC plus 10\% slag; 1) OPC plus 30\% slag; m) OPC plus 50\% slag 182 


\section{Introduction}

\subsection{Background}

The structural and durability performance of concrete depends on its hardened properties such as compressive strength and transport properties. These hardened properties depend on concrete's mixture design (e.g. cementitious material content, water-to-cementitious material ratio or w/c) and fresh properties (e.g. slump, air content). For a given concrete mixture design, the fresh properties have the most significant influence on hardened and durability properties. In recent years, the electrical resistivity of fresh concrete has gained increasing attention as a performance index because of its practicality and the extent of information it potentially provides on fresh properties. However, since electrical resistivity of concrete is affected by a wide range of parameters related to the mixture design, it is not trivial to relate electrical resistivity of fresh concrete to other performance parameters. In general terms, this research provides a comprehensive investigation on the relationship between the electrical resistivity of concrete with various mixture design properties.

Among two main components of fresh concrete, paste and aggregates, the former affects the electrical characteristics of concrete more than the latter, especially in the fresh state. The electrical resistivity of aggregates is much higher than that of the paste [1]; therefore, for practical purposes, they can be considered nonconductive within fresh concrete. Therefore, the focus of the current study is only on the fresh cement paste before setting time. 


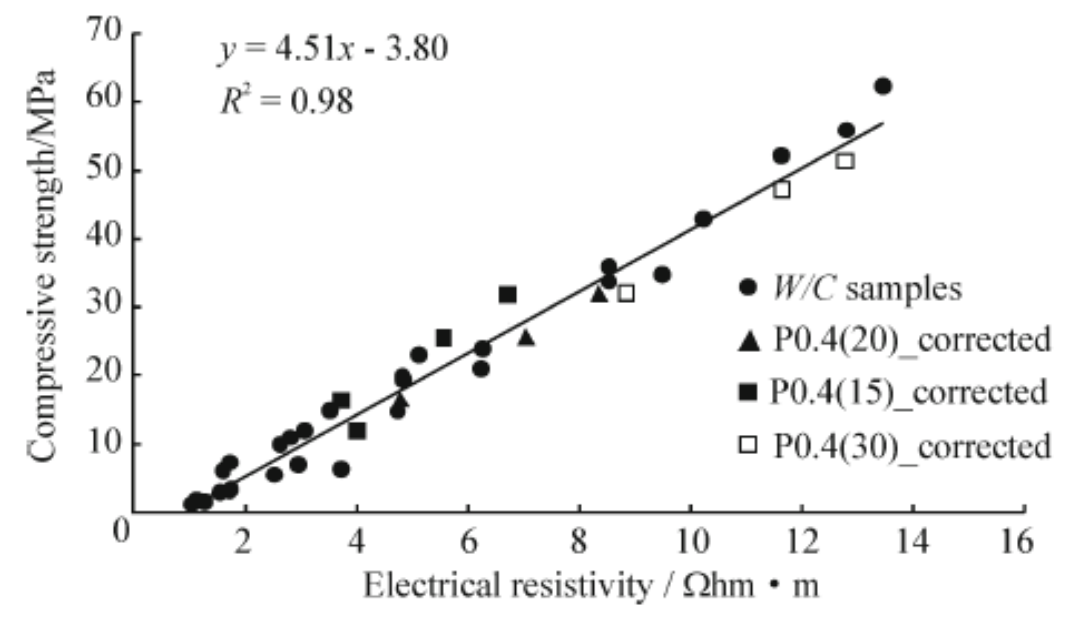

Figure 1.1. Relationship between the compressive strength and the electrical resistivity for pastes with different $\mathrm{w} / \mathrm{c}$ ratios and different curing temperatures of 15,20 and $30{ }^{\circ} \mathrm{C}$ [2].

Electrical resistivity measurements have been known to characterize the concrete behaviour as a non-destructive test since 1950s [3]. In recent years, the number of investigations on the subject has increased dramatically [4-15] For example, Xiao et al. [2] reported that the compressive strength of hardened paste at early age can be estimated accurately from associated electrical resistivity measurements, as shown in Fig. 1.1.

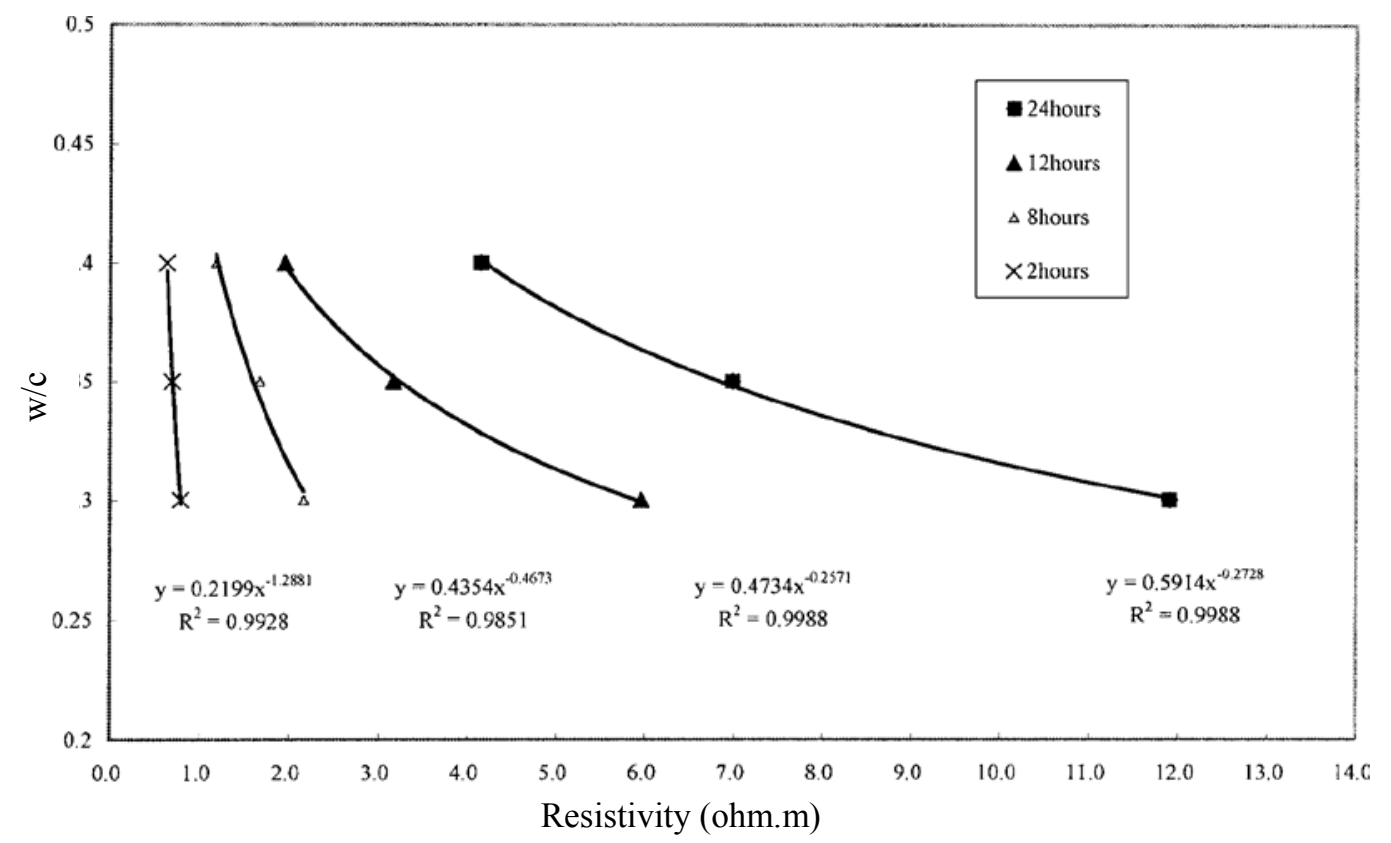

Figure 1.2. Relationship between w/c and electrical resistivity of pastes at early age [14]. 
Similarly, the effect of w/c on electrical resistivity of cement paste has also been investigated by a number of researchers $[5,14,16]$. For example, Li et al. [14] suggested relationships between $\mathrm{w} / \mathrm{c}$ ratio and electrical resistivity of cement paste as illustrated in Fig.1.2, which shows that at a fixed hydration age, a lower w/c ratio corresponds to a higher bulk resistivity of paste. However, the investigation did not consider the fresh state during first two hours and before setting initiates. Similar problem exists in other studies $[7,17]$ which mainly focus on electrical properties of concrete close to setting times and during hardening. Since electrical characteristics of the paste change with time due to ongoing chemical (hydration) reactions and microstructure development, the very fresh stage, during which the paste does not enter setting and subsequently hardening period (mostly during first 2 hours), requires separate broad study which lacks in literature.

The Supplementary Cementitious Materials (SCM) and chemical admixtures (e.g. superplasticizers) are widely used to improve the properties of ordinary portland cement (OPC). Some of the advantages of these materials include improved workability, lowered cost, improved resistance to external attack in aggressive environments, and reduced heat of hydration and thermal shrinkage. Currently, the most commonly used SCMs are fly ash, silica fume and ground granulated blast furnace slag (henceforth, slag). The effect of these SCMs and chemical admixtures on the electrical resistivity of concrete or cement paste has been studied by some researchers [8-10, 15, 18]. For instance, Salem [9] observed that increasing silica fume replacement in OPC pastes decreases the conductivity at the early stage as shown in Fig. 1.3. 


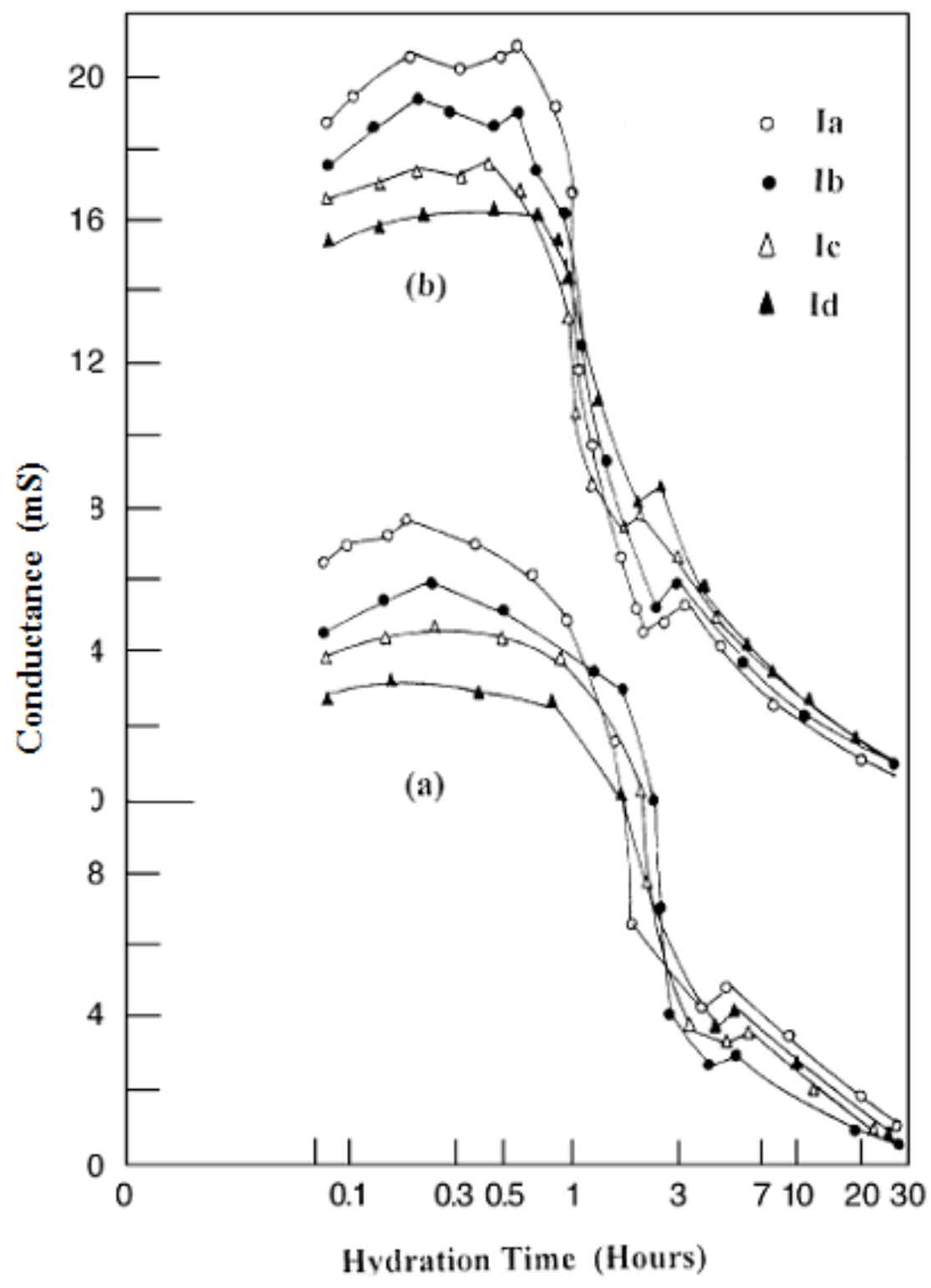

Figure 1.3. Conductance-Time curves of OPC plus silica fume paste mixtures. Ia, Ib, Ic and Id represent 10, 20, 30 and 50 percent silica fume replacement, respectively; for w/c of 0.70 at two temperatures: (a) $25^{\circ} \mathrm{C}$; and (b) $45^{\circ} \mathrm{C}$ [9].

It should be noted that the electrical resistivity of cement paste is mainly determined by its liquid phase (i.e., the pore solution), which is considered to be orders of magnitude 
more conductive than its solid phase (cementitious materials) [16]. Hence, studying the electrical resistivity of cement paste requires the study of pore solution conductivity/resistivity, which is a complex problem due to the laborious nature of the pore solution extraction process from the cement paste. A few studies have been carried out to study the pore solution electrical conductivity along with associated paste types [5, 11, 12]. For example, Sant et al. [11] monitored the pore solution electrical conductivity during first 48 hours of mixing for only OPC paste with fixed w/c of 0.3 as shown in Fig. 1.4. However, the effect of the w/c and the incorporation of the SCMs was not studied. In general, no broad investigation was conducted on pore solution of different types of pastes, with and without SCMs and chemical admixtures, during very early stages after mixing (e.g. first 2 hours). Further research on the subject is required.

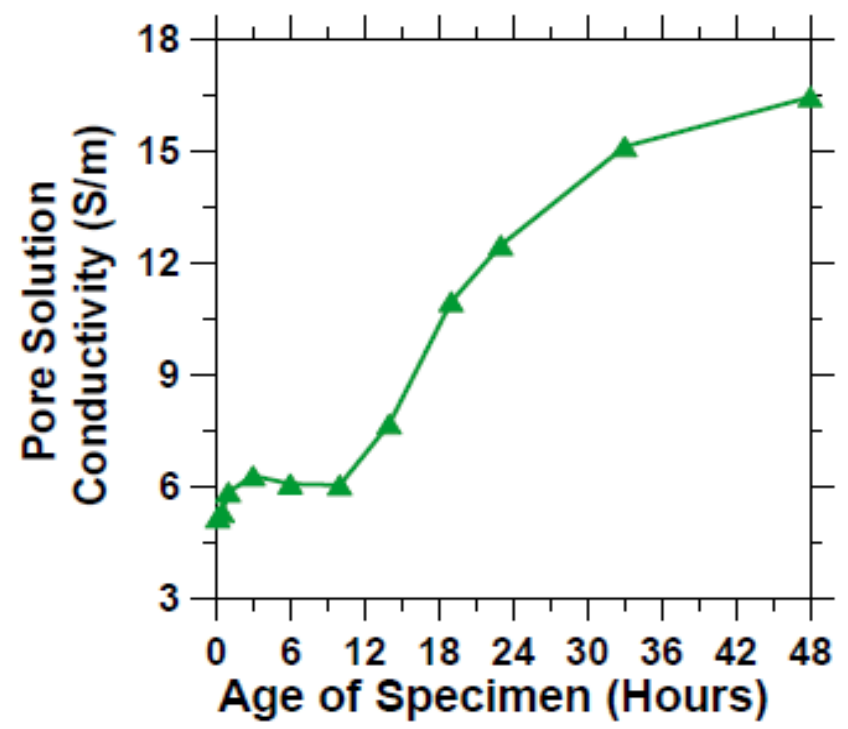

Figure 1.4. Pore solution conductivity versus paste specimen age for a paste with w/c of $0.3[11]$ 
In Archie's law [19] the formation factor is defined as the ratio of electrical resistivity of sand stones $100 \%$ saturated with water to that of water contained in the pores which is a function of volumetric ratio of pores, i.e., porosity. Some researchers investigated applicability of Archie's law in cement paste in order to estimate porosity and subsequently w/c of paste from formation factor . For example, Li et al. [5] extracted the pore solution of three OPC pastes with w/c of $0.3,0.4$ and 0.5 at fresh state to establish the formation factor-porosity relationship, as shown in Fig. 1.5. They showed that the formation factor of cement paste decreased with increasing w/c or porosity. However, the effect of chemical admixtures or SCMs was not studied and only three w/c ratios were considered in their study. Therefore, further investigation on Archie's law in different cement paste mixtures was also included in this thesis.

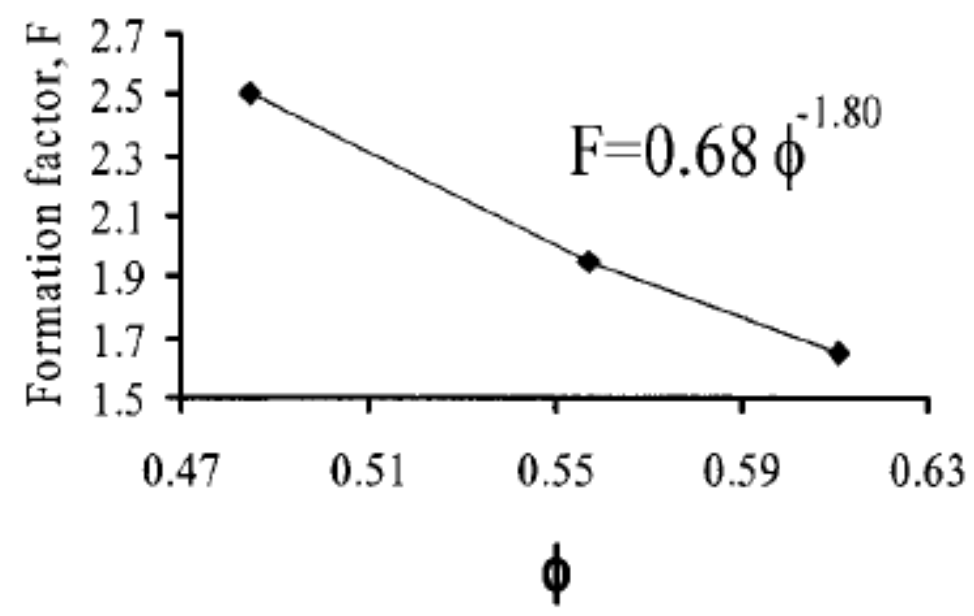

Figure 1.5. Formation factor of OPC pastes as a function of porosity [5]. 


\subsection{Problem Definition}

Electrical resistivity of a cement paste at fresh state which is dominated by electrical properties of its pore solution, is concurrently affected by a number of factors as follows: w/c ratio which is designed in the mixture properties; the time of resistivity measurement which reflects the hydration progress and thus the concentration of ions released in pore solution; the types and associated physical and chemical properties of materials used in the mixture (i.e., OPC, SCMs, water, and chemical admixtures); the dosages of SCMs and chemical admixtures used in the mixture properties; size, shape and distribution of solid particles (cementitious materials) in the pore solution; and the temperature of resistivity measurement. Therefore, even if an investigation focuses on a particular parameter (e.g. w/c ratio) affecting the electrical resistivity, other influential factors should also be taken into account because of simultaneous impact of them. Many researches have been conducted to study these effects as well as correlation of electrical resistivity of paste to its characteristics (e.g. compressive strength, setting times); however, they were not comprehensive enough and some contradictions were found in the literature, specially on the effect of w/c ratio on the cement paste electrical resistivity during fresh state. In addition, the laborious process of pore solution extraction for experimental measurement of electrical resistivity marks the considerable demand for a numerical model to estimate the conductivity of pore solution during fresh state. However, the numerical approach has not been accurate for pastes in the fresh state.

In particular, the following gaps in the literature that define the problem addressed in this thesis are identified: 
- Although the effect of $\mathrm{w} / \mathrm{c}$ on paste resistivity was studied in a limited number of researches, specifically at fresh state, as it will be demonstrated in the literature review, there has been some disagreement with the reported conclusions.

- The pore solution conductivity development with time during fresh state has not been studied systematically, and the researchers focused more on later stages (e.g. during setting and hardening). In addition, no numerical model has been proposed that estimates pore solution conductivity with respect to time before setting initiates.

- There is no study comprehensive enough to consider the effect of type, various dosages, and physical properties (e.g. size and distribution, hydrodynamic viscosity) of the most commonly used SCMs (e.g. fly ash, silica fume and slag) and admixtures (e.g. superplasticizer) on the electrical resistivity of pore solution and paste at fresh state.

- Applicability of the Archie's law in cement paste was studied only by a few researchers; however, they were not broad enough to cover the wide variety of w/c ratios and associated admixtures and SCMs in concrete.

- No research has been conducted on the correlation between the $\mathrm{pH}$ and conductivity of pore solution at fresh state as an alternative measurement method.

\subsection{Objective and scope}

The overall objective of the current research is to investigate the gaps identified in literature regarding electrical resistivity measurements of fresh cement pastes and thus the scope of work conducted in this research is limited to fresh state. Specifically, the 
main objective of this thesis is to evaluate the feasibility of the use of electrical resistivity measurements as an in-situ technique for the determination of $w / c$ ratio of fresh concrete before or during pouring into formworks. Even though the w/c ratio is a critical parameter in quality control of concrete structures and determines the main strength and durability properties of hardened concrete, there is no in-situ method available for its prediction during construction; therefore, there is a considerable demand for such a tool by the construction industry. Eventually, the outcome of this research might result in the development of an affordable and easy-to-use handheld device for field application that correlates the ratio of fresh cement paste electrical resistivity to that of pore solution obtained from proposed model, formation factor $\mathrm{F}$, to its $\mathrm{w} / \mathrm{c}$ ratio. This development will provide engineers with a powerful tool to improve the quality of concrete used in new structures throughout the world.

To achieve the above mentioned objective, a comprehensive experimental plan and a numerical model were developed to estimate the pore solution conductivity at fresh state. The effect of the w/c on pore solution and paste resistivity was explored for a wide range of w/c (i.e., from 0.30 to 0.55 ). The effect of the type and amount of chemical admixtures and SCMs in the paste mixture was extensively investigated; i.e., OPC pastes incorporated with superplasticizer, fly ash, silica fume and slag with three different dosages (low, medium and high) were included in the study. During the first 2 hours of paste age, three different time benchmarks (e.g. $30^{\text {th }}, 60^{\text {th }}$ and $90^{\text {th }}$ minute) were selected to investigate the time effect on the electrical resistivity. Temperature was also monitored to normalize all the measured data such as resistivity and $\mathrm{pH}$ to a reference temperature of $25{ }^{\circ} \mathrm{C}$. In addition, a reliable analytical model was proposed to predict the pore 
solution conductivity with respect to time for different types of paste mixtures at fresh state before the setting time.

\subsection{Organization of the Thesis}

The chapters of this thesis are arranged as following: Chapter 1 provides a background on the research and presents the objectives and scope of the study; Chapter 2 reviews the previous studies on the electrical resistivity evaluation of cement-based materials and inferential parameters as well as the gaps in literature; Chapter 3 describes the experimental procedure conducted in the research; Chapter 4 presents the selected results and discussions on the experimental data; Chapter 5 proposes a numerical model to approximate the pore solution conductivity during fresh state; Chapter 6 presents the conclusions and provides recommendations for future studies; Appendix A defines some technical terms used in the area of electrical resistivity; and Appendix B presents the supplementary results on the experimental data which are not given in Chapter 4 because of brevity. 


\section{Literature Review}

\subsection{Introduction}

Electrical resistivity of concrete provides valuable information for engineers. Because it not only reflects the chemical reactions occurring between cementitous materials and water in concrete, but also indicates the physical properties and microstructure of its components (i.e., cementitious materials, water, and aggregates). For instance, lower electrical resistivity or higher electrical conductivity of concrete shows its higher vulnerability to aggressive ion penetration such as $\mathrm{Cl}^{-}$in an aggressive environment. Therefore, the durability of such concrete decreases which is an important criterion to meet the goals of sustainable development. Also, as discussed in Chapter 1, the mechanical properties of hardened concrete such as compressive strength were shown to have a strong correlation to its electrical resistivity; i.e., the higher the electrical resistivity of hardened concrete, the higher the compressive strength. Hence, the electrical resistivity measurement can be utilized as an in-situ method to determine the characteristics of concrete.

The properties of hardened concrete such as permeability and compressive strength are significantly determined by its features at fresh state such as water-to-cementitious materials ratio, setting times or slump. Therefore, many investigations have been conducted to study the electrical resistivity of concrete at early stage and fresh state. Electrical resistivity of cement-based materials such as concrete, mortar or cement paste is affected by different criteria. Because of the cement hydration progress, the physical and chemical composition of concrete change over time; the temperature changes ions 
mobility in the pore solution; the types of cementitious materials changes the rate of hydration and amount of ions released in the pore solution, the water-to-cementitious materials $(\mathrm{w} / \mathrm{c})$ affects the proportions of the pore solution compared to the solid particles with different orders of electrical resistivities; and the type of measurement technique used to determine the electrical resistivity of concrete, mortar or cement paste can be the source of some inherent errors which can affect the accuracy of the measurements. Hence, the effect of all these factors on the electrical resistivity of fresh concrete should be taken into account to characterize appropriately the properties of fresh concrete.

\subsection{Application of electrical resistivity for fresh concrete}

Cement paste is the most significant component of concrete in the determination of the characteristics of hardened concrete and because of hydration process the cement paste characteristics are time dependent. In a same way the electrical resistivity of the cement paste also varies with time. Thus, electrical resistivity measurement can be used as a strong tool to estimate the characteristics of the cement paste such as its $w / c$ or setting time. These characteristics practically are very important in construction industry, specifically at early age of hydration.

\subsubsection{Setting Time}

The setting time is an important factor in quality control of concrete. A desirable setting time should be long enough to provide the time for mixing, transporting, casting and finishing of fresh concrete for the construction crew. Typically, the initial and final setting times of concrete are measured by the penetration method that has been 
standardized in ASTM C 403 [20]. The test is done on mortar which has been sieved from the fresh concrete. The initial setting time $\left(\mathrm{t}_{\mathrm{i}}\right)$ of concrete corresponds to the ending of plasticity state while the final setting time $\left(t_{f}\right)$ of concrete corresponds to onset of hardening. The initial and final setting time are determined by the times at which the penetration resistance reaches 3.5 and 26.7 $\mathrm{MPa}$, respectively, when a designated needle penetrates $25.4 \mathrm{~mm}(1 \mathrm{in})$ into the mortar. There are some difficulties associated with this test such as extracting the mortar from concrete, time consuming test process and variance of results by different operators. However, the electrical resistivity measurement eliminates all above mentioned practical problems because it is done on concrete itself rather than mortar; it also continuously measures data immediately after mixing.

The setting times of cement were studied using electrical technique as early as 1930s [21]. The retardation effect of superplasticizer incorporated pastes was later investigated $[22,23]$. McCarter et al. [24] also used electrical resistivity measurement technique to monitor setting and hardening times.

Li et al. [6] used a non-contact electrical resistivity measurement [4] to estimate the setting times of concrete as a function of minimum point $\left(\mathrm{P}_{\mathrm{m}}\right)$ and the transition point $\left(\mathrm{P}_{\mathrm{t}}\right)$ on resistivity-time curves as shown in Fig. 2.1. $\mathrm{P}_{\mathrm{m}}$ represents the point on $\rho$-t curve which has minimum resistivity value that correlates to maximum conductivity. $\mathrm{P}_{\mathrm{t}}$ on the other hand is defined on logarithmic scale $\rho$-t curve as the point located at maximum curvature that describes the transition in fresh concrete from setting to hardening and gaining strength. In Fig. 2.1, Mix 1, Mix 10 and Mix 3 represent concrete samples with the w/c of $0.3,0.4$ and 0.3 containing $0.8 \%$ superplasticizer, respectively. 


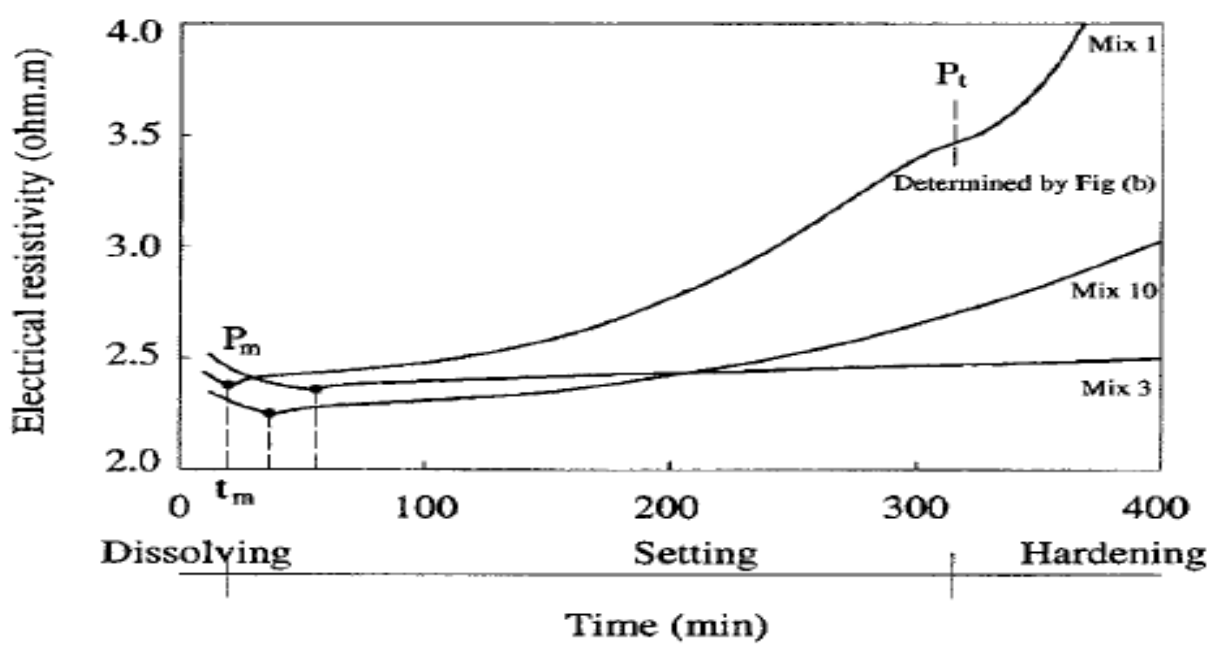

(a)

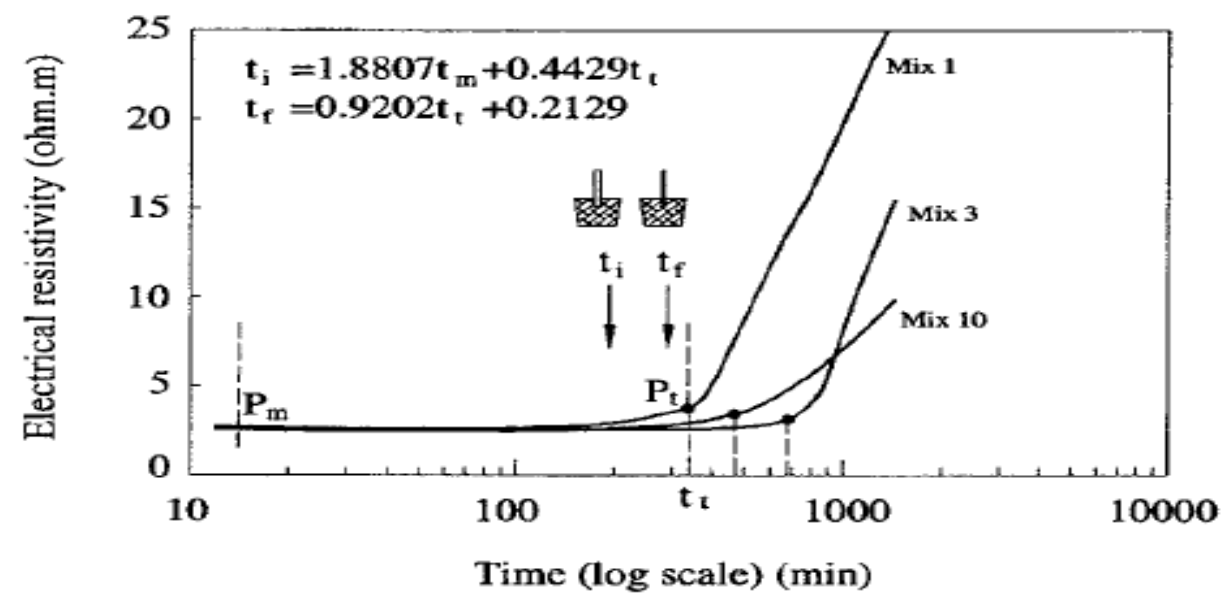

(b)

Figure 2.1. Electrical resistivity development of concretes to identify: (a) minimum point $\mathrm{P}_{\mathrm{m}}$ on $\rho$-t curves; (b) transition point $\mathrm{P}_{\mathrm{t}}$ on $\rho$-t (log scale) curves [6].

Li et al. [6] found the setting times of concrete samples by penetration method [20] along with their minimum time $t_{m}$ and transition time $t_{t}$ from resistivity measurement. These results are presented in Table 2.1. Using the regression analysis, they proposed a relationship between critical points and setting times derived from the penetration test. 
The initial and final setting times were quantified as a function of the time of onset of hydration $\left(\mathrm{t}_{\mathrm{m}}\right)$ and time at which the transition point $\left(\mathrm{t}_{\mathrm{t}}\right)$ occurs as follows:

$t_{i}=1.8807 t_{m}+0.4429 t_{t}, \quad R^{2}=0.8950$

$t_{f}=0.9202 t_{t}+0.2129, \quad R^{2}=0.9895$

where $t_{i}$ and $t_{f}$ are the initial and final setting times, respectively.

Table 2.1. Times of occurrence of critical points $t_{m}$ and $t_{t}$ in resistivity response and the setting times of concrete resulted from penetration test [6].

\begin{tabular}{lcccccc}
\hline & & \multicolumn{2}{c}{ Resistivity response } & \multicolumn{2}{c}{ Setting time (ASTM C 403) } \\
\cline { 3 - 4 } \cline { 6 - 7 } Mix & & $\begin{array}{c}\text { Point } P_{m} \\
\left(t_{m}\right)\end{array}$ & $\begin{array}{c}\text { Point } P_{t} \\
\left(t_{t}\right)\end{array}$ & & $\begin{array}{c}\text { Initial } \\
\left(t_{i}\right)\end{array}$ & $\begin{array}{c}\text { Final } \\
\left(t_{f}\right)\end{array}$ \\
number & Name & $(\mathrm{h})$ & $(\mathrm{h})$ & & $(\mathrm{h})$ & $(\mathrm{h})$ \\
\hline 1 & Control & 0.33 & 5.08 & 3.5 & 4.73 \\
2 & Sp1-0.6 & 0.70 & 8.35 & 5.82 & 8.02 \\
3 & Sp1-0.8 & 0.93 & 11.77 & 8.4 & 11.77 \\
4 & Sp1-1 & 1.62 & 13.45 & 9.42 & 13.12 \\
5 & Sp2-0.15 & 1.02 & 11.58 & 7.22 & 11.4 \\
6 & Sp2-0.25 & 1.27 & 15.92 & 9.38 & 14.2 \\
7 & Sp2-0.35 & 2.07 & 17.95 & 11.83 & 16.8 \\
8 & Accelerator & 0.24 & 3.98 & 2.91 & 3.85 \\
9 & W-C0.5 & 0.90 & 10.90 & 4.58 & 10.23 \\
10 & W-C0.4 & 0.62 & 7.70 & 3.75 & 6.88 \\
11 & W-C0.4 & 0.73 & 8.03 & 3.83 & 7.83 \\
& FA cement & & & & \\
\hline
\end{tabular}


Li et al. [14], using the electrical resistivity development curve and corresponding rate of electrical resistivity shown in Fig. 2.2, suggested that setting period (II) starts at initial setting time, $t_{m}$, in which the resistivity of the paste is minimum and after this point it increases slowly because of the formation of Ettringite, $\mathrm{CH}$ and $\mathrm{CSH}$ up to the point that suddenly the rate of increase in resistivity $(\mathrm{d} \rho / \mathrm{dt})$ considerably grows, $\mathrm{t}_{\mathrm{a}}$, that is indicative of the final setting time and beginning of hardening.

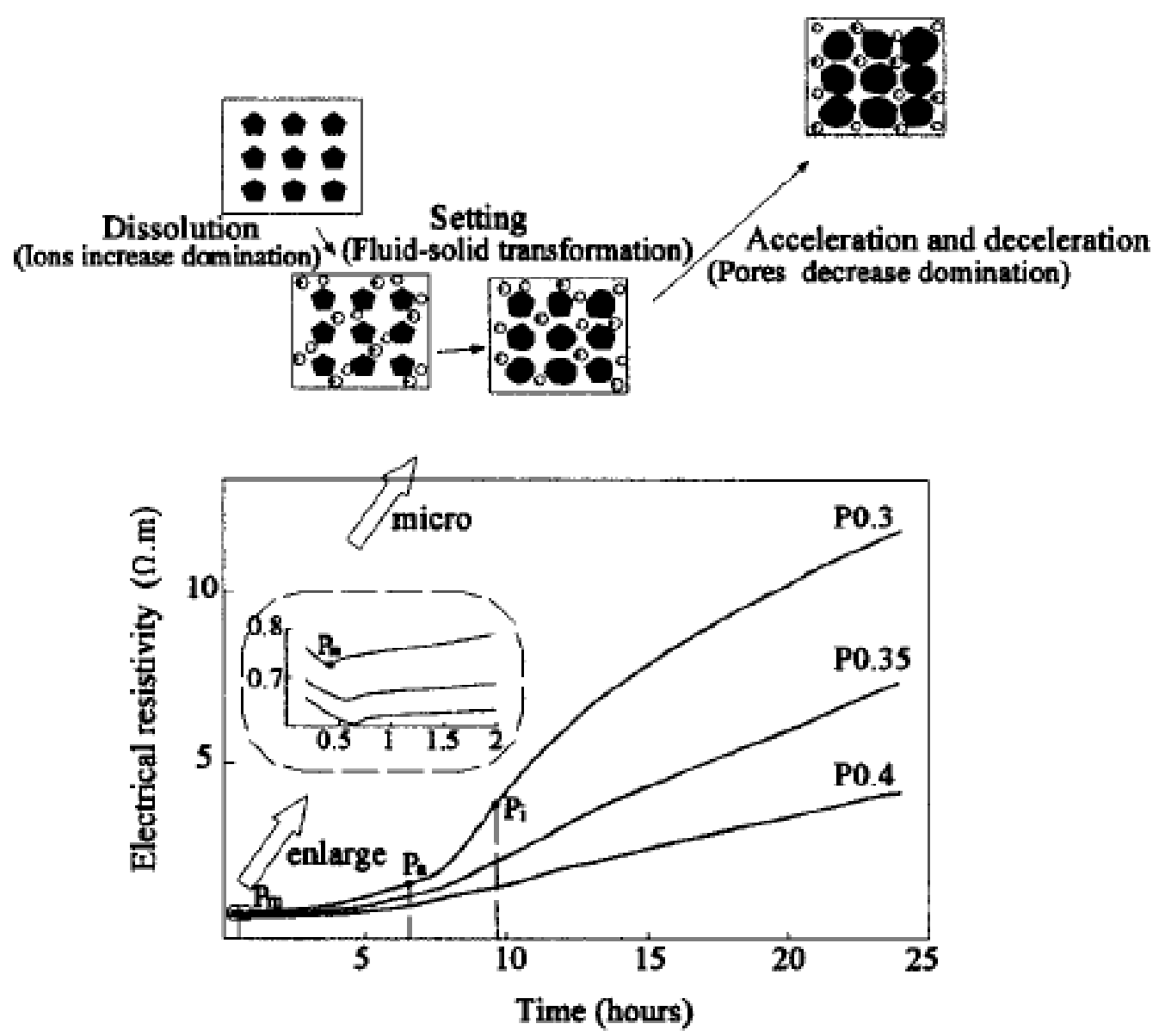

(a) 


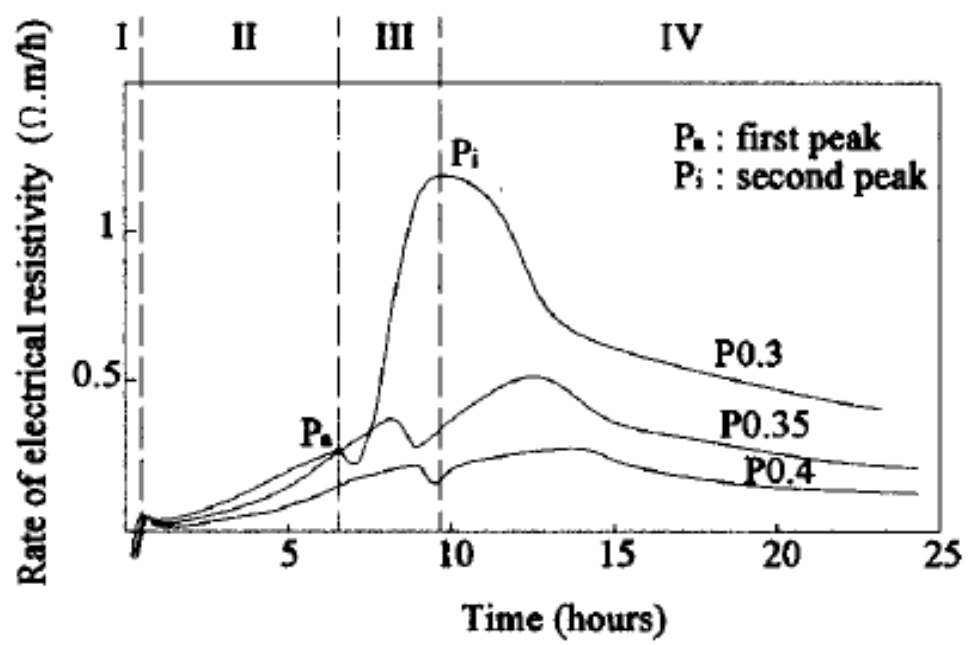

(b)

Figure 2.2. Curves of different pastes with $w / c$ of $0.3,0.35$ and 0.4 denoted by $\mathrm{P} 0.3$, P0.35 and P0.4; respectively, over 24 hrs: (a) Resistivity development; (b) Rate of resistivity development [14].

Furthermore, in another study based on resistivity-time curves for different w/c pastes, Li et al. [5] suggested four stages of hydration shown in Fig. 2.6 as dissolving period (I), a competition period (II), a setting (III) and hardening period (IV). These different stages were defined by indicating specific points as their boundaries on $\rho$-t curves. $M$ $(\mathrm{t}(\mathrm{m}), \rho(\mathrm{m}))$ represents the minimum critical point; $\mathrm{L}(\mathrm{t}(\mathrm{l}), \rho(1))$ indicates the point in time at which almost level (plateau) curve ends and resistivity starts to rise markedly and I $(t(i), \rho(i))$ shows the point of inflection at which concavity changes from upward to downward; i.e., the second derivative of resistivity with respect to time becomes zero. 


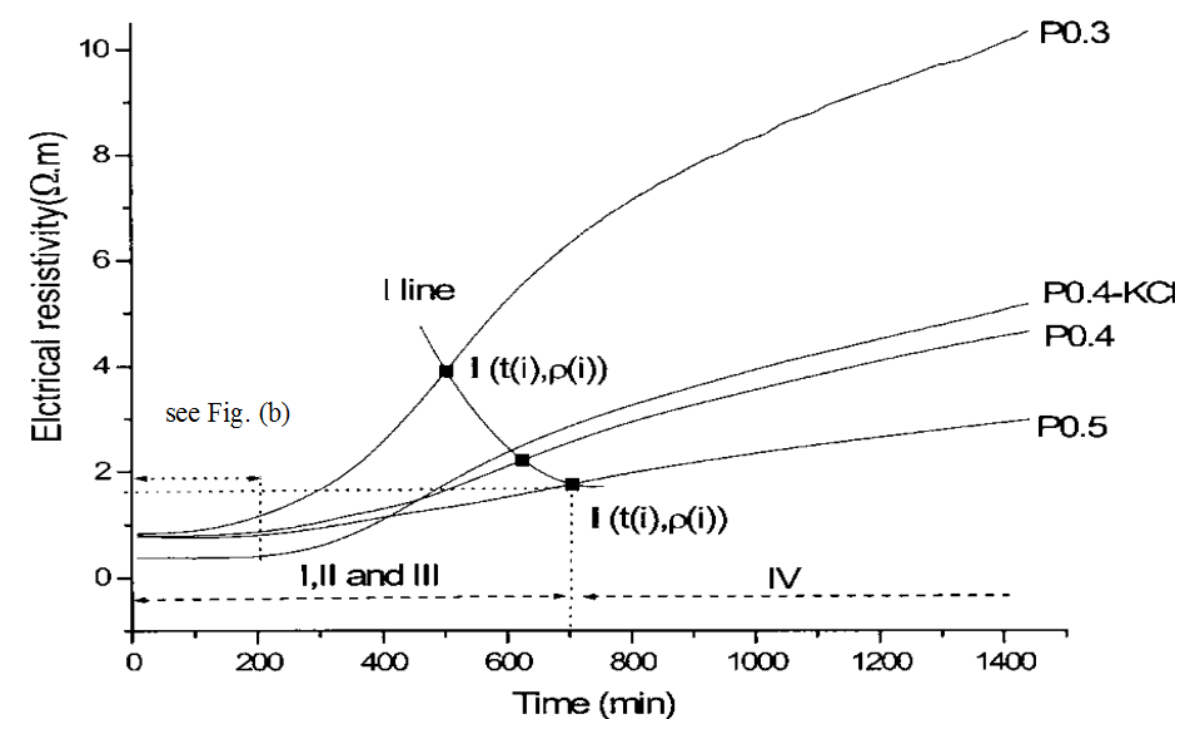

(a)

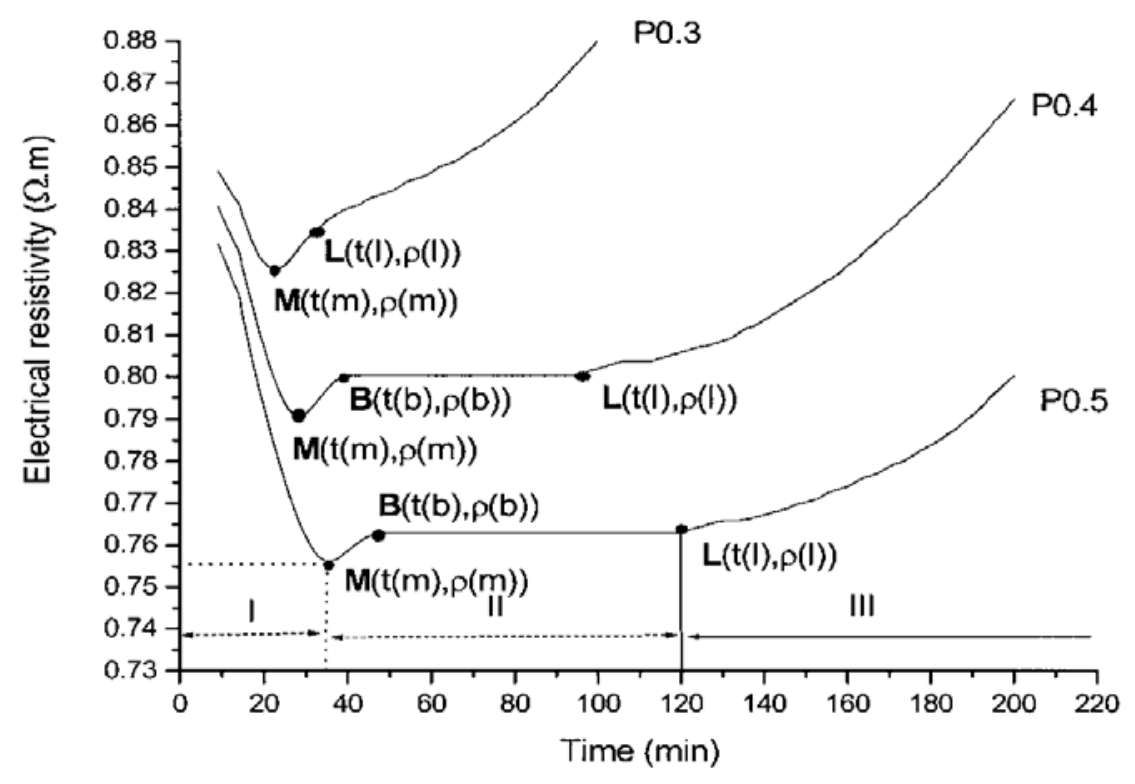

(b)

Figure 2.3. Resistivity development versus time for paste with different w/c ratios; i.e., P0.3, P0.4 and P0.5 are paste samples with w/c of $0.3,0.4$ and 0.5 , respectively; while $\mathrm{P} 0.4-\mathrm{KCl}$ is a paste incorporated with $1 \% \mathrm{KCl}$ by mass in cementitous materials: a) during first 1440 minutes; b) during first 200 minutes [5]. 
Bekir at al. [13] conducted the Vicat needle test to determine the initial and final setting time of pastes with and without SCMs such as fly ash, silica fume and slag. They reported that in general, the final setting time of pastes containing SCMs increased compared to that containing only OPC. This increase was mainly because of reduction in $\mathrm{C}_{3} \mathrm{~A}$ component in the binder which exists in cement and plays a significant role in early hydration; i.e., the less the $\mathrm{C}_{3} \mathrm{~A}$ hydrated products, the longer time it takes for paste to gain minimum strength for resisting to Vicat needle. On the other hand, although the initial setting time in fly ash and slag incorporated pastes showed slightly increase, the silica fume added pastes were observed to have noticeable increase. This different behavior in silica fume-containing pastes was related to the surface area (see Table 2.2) of silica fume (i.e., $14,000 \mathrm{~cm}^{2} / \mathrm{g}$ ) compared to that of OPC, fly ash, and slag (BFS) which were 3312,3126 , and 4982 , respectively. The water left for hydrating the cement decreases in the presence of much larger surface area due to higher absorption and as a result, cement particles hydration is retarded.

Table 2.2. Physical and chemical properties of OPC and supplementary cementitious materials [13].

\begin{tabular}{lllll}
\hline Component (\%) & OPC & BFS & FA & SF \\
\hline $\mathrm{CaO}$ & 63.6 & 39.8 & 6.66 & 1.48 \\
$\mathrm{SiO}_{2}$ & 19.6 & 32.8 & 47.4 & 74.7 \\
$\mathrm{Al}_{2} \mathrm{O}_{3}$ & 4.72 & 11.8 & 19.8 & 0.46 \\
$\mathrm{Fe}_{2} \mathrm{O}_{3}$ & 3.27 & 1.45 & 11.8 & 0.84 \\
$\mathrm{MgO}$ & 1.91 & 4.15 & 4.76 & 3.64 \\
$\mathrm{Na}_{2} \mathrm{O}$ & 0.34 & 0.51 & 0.57 & 0.85 \\
$\mathrm{~K}_{2} \mathrm{O}$ & 1.06 & 0.91 & 2.62 & 5.05 \\
$\mathrm{SO}_{3}$ & 4.72 & 2.06 & 1.86 & 2.48 \\
$\mathrm{Cr}_{2} \mathrm{O}_{3}$ & 0.04 & 0.02 & 0.13 & 2.83 \\
$\mathrm{TiO}_{2}$ & 0.41 & 0.63 & 0.88 & 0.63 \\
$\mathrm{LOI}^{\text {Specific gravity }}$ & 2.69 & 2.2 & 2.76 & 5.97 \\
Fineness $\left(\mathrm{cm}^{2} / \mathrm{g}\right)$ & 3.07 & 2.8 & 1.99 & 2.44 \\
\hline
\end{tabular}




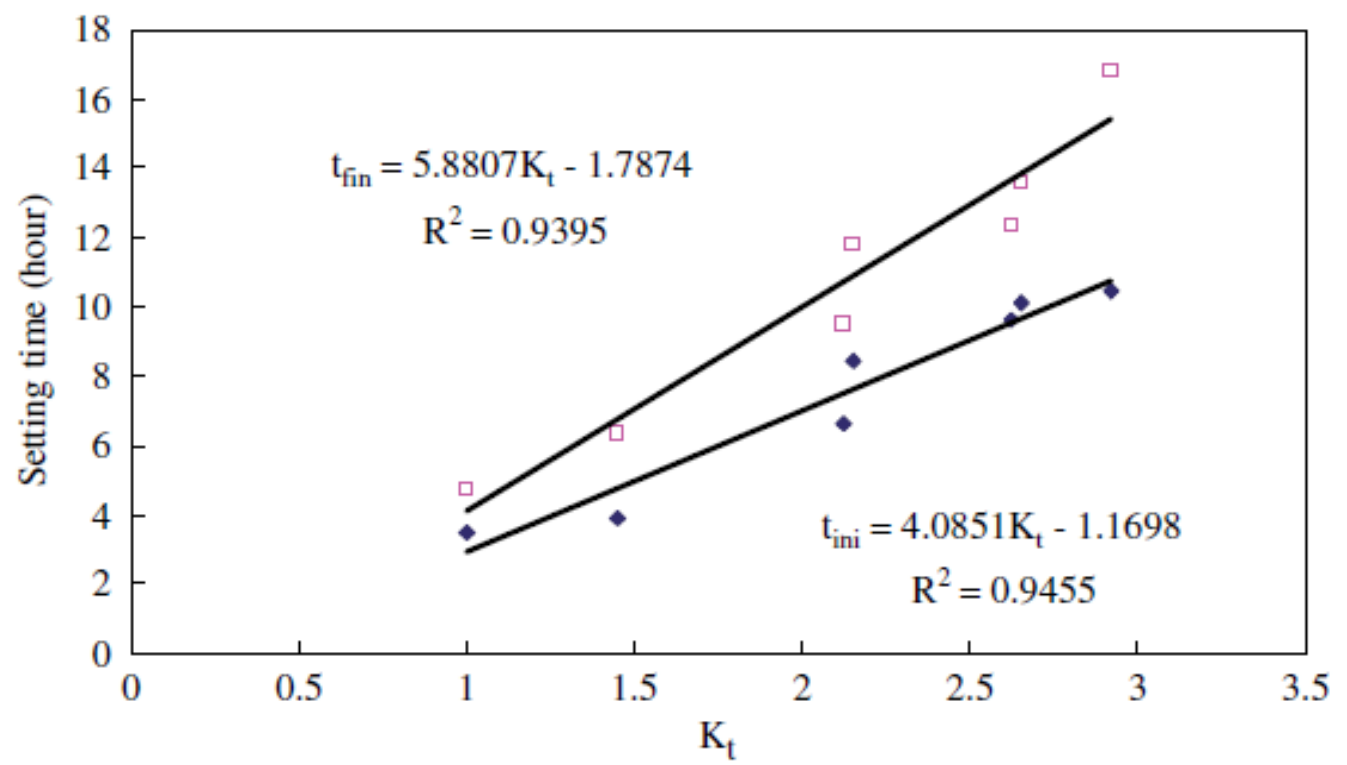

Figure 2.4. Correlation of setting time $\left(t_{\text {ini }}, t_{\text {fin }}\right)$ resulted from penetration test and inflection time ratio $K_{t}[25]$. (Note: Squares and diamonds show final and initial setting times, respectively).

Xiao et al. [25] concluded that the proposed inflection time ratio $\mathrm{k}_{\mathrm{t}}$ strongly correlates to initial and final setting times (defined by penetration test) of superplasticizer incorporated pastes with different dosages. This correlation was observed to be linear as shown in Fig. 2.4, and can be used to estimate setting times of concrete samples containing superplasticizer via:

$t_{\text {ini }}=a K_{t}+b$

$t_{f i n}=c K_{t}+d$

where $t_{\text {ini }}$ and $t_{\text {fin }}$ are the initial and final setting times, respectively; and $a, b, c$, and $d$ are the regression analysis constants. 


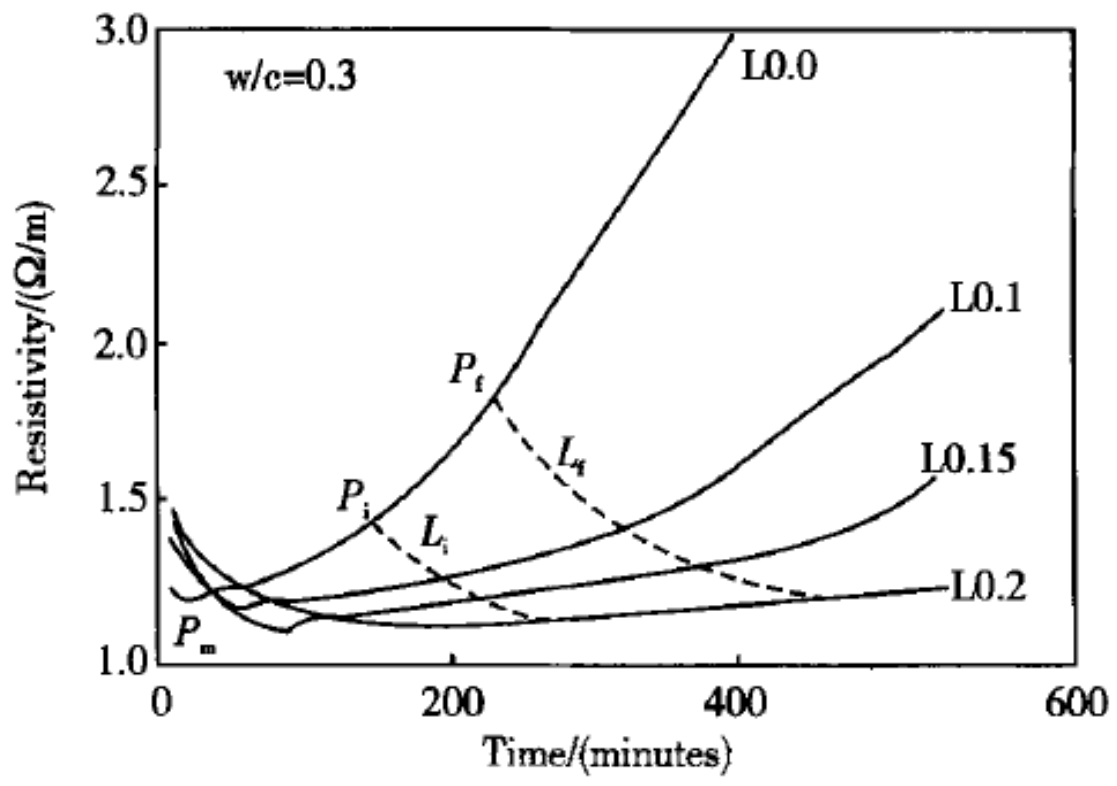

Figure 2.5. Electrical resistivity development of pastes with the w/c of 0.3 and containing $0 \%, 0.1 \%, 0.15 \%$ and $0.2 \%$ retarder denoted by L $0.0, \mathrm{~L} 0.1, \mathrm{~L} 0.15$ and $\mathrm{L} 0.2$, respectively [26].

In addition, $\mathrm{Li}$ et al. [26] monitored the resistivity development of cement pastes incorporated with retarder of different dosages $0 \%, 0.1 \%, 0.15 \%$ and $0.2 \%$ (by weight with respect to the solid content) at the w/c ratio of 0.3 during first 24 hours. They concluded that the retarders delayed the development of electrical resistivity curve and consequently the initial and final setting times increased (Fig. 2.5). 


\subsubsection{Water-to-Cementitious Materials Ratio (w/c)}

The w/c ratio of concrete considerably affects physical and mechanical properties of hardened concrete. Even though the w/c ratio is a critical parameter in quality control of concrete structures and the strength gain and durability properties of hardened concrete, there is no reliable in-situ method available for its prediction during construction; therefore, there is a considerable demand for this in the construction industry.

Li et al. [14] reported that higher w/c ratio can prolong the setting period and also delays the setting times of pastes (see Fig. 2.2). At each specific age of hydration, they suggested that relationship between resistivity of paste and w/c ratio followed a power trend as $\mathrm{Y}=\mathrm{Ax}{ }^{\mathrm{B}}$. The exponent $\mathrm{B}$ was always negative and it illustrated that at a fixed hydration age, a lower w/c ratio corresponded to a higher bulk resistivity (Fig. 2.2). Whittington et al. [16] also reported that increasing w/c ratio in concrete and cement paste resulted in decreasing of electrical resistivity and strength subsequently.

Li et al. [5] concluded that bulk resistivity of the pastes depends on w/c ratio and lower w/c pastes always have higher resistivity than that for higher w/c pastes as shown in Fig. 2.3. This conclusion was valid at any age in hydration process and is schematicly demonstrated in Fig. 2.6.

In 2007, $\mathrm{Li}$ et al. [6] observed that increasing w/c ratio increases both initial and final setting times of concrete mixtures (Table 2.1). 


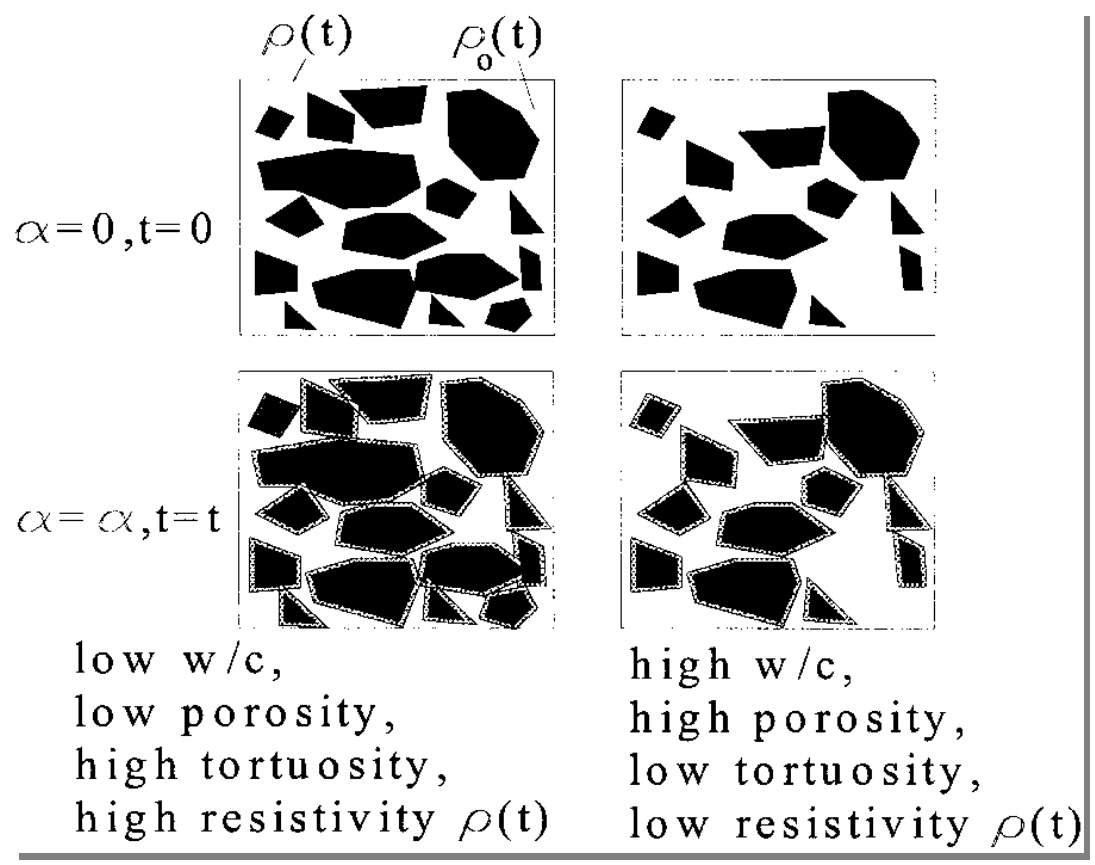

Figure 2.6. Schematic representation of cement paste structure with time $\mathrm{t}$ and corresponding hydration degree $\alpha[5]$.

Bekir at al. [13] found that for all the paste mixtures including paste without SCMs and pastes with SCMs such as fly ash, silica fume and blast furnace slag, increasing the water/binder (OPC plus SCMs) increases the electrical conductivity (decreases the electrical resistivity). Furthermore, rate of decrease in electrical conductivity is more for lower water/binder ratios than that of higher $\mathrm{w} / \mathrm{c}$ ratios.

Wei and Xiao [27] reported that for the fixed aggregate volume fraction $\mathrm{V}_{\mathrm{a}}$, the concrete samples with lower $\mathrm{w} / \mathrm{c}$ ratios have higher electrical resistivity than that of greater $\mathrm{w} / \mathrm{c}$ ratios concrete (see Fig. 2.7). In addition, the rate of electrical resistivity rise with time $(\Omega . \mathrm{m} / \mathrm{h})$ is higher for the lower $\mathrm{w} / \mathrm{c}$ ratio concrete which is attributed to the less free water and more concentration of ions in hydration system to form hydration products compared to higher $\mathrm{w} / \mathrm{c}$ ratio concrete. 


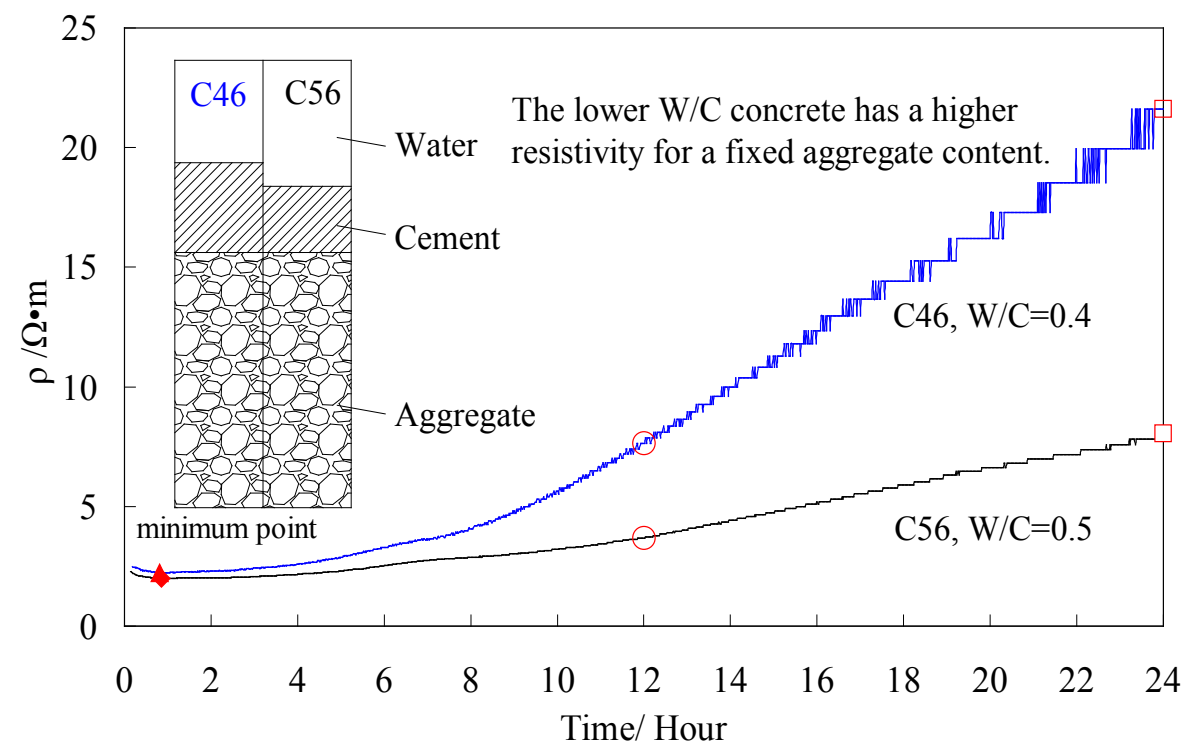

Figure 2.7. Electrical resistivity versus time of hydration for two concrete samples with aggregate volume fraction of $60 \%$ [27].

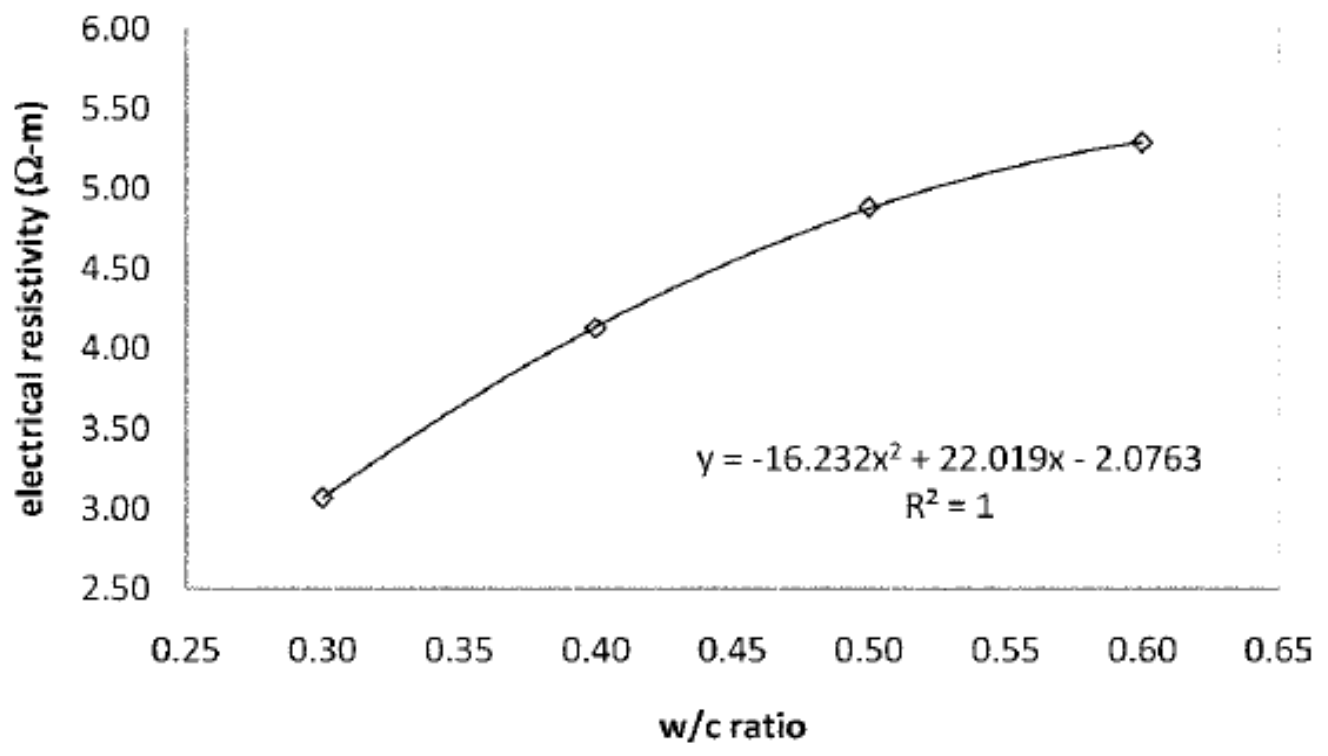

(a) 


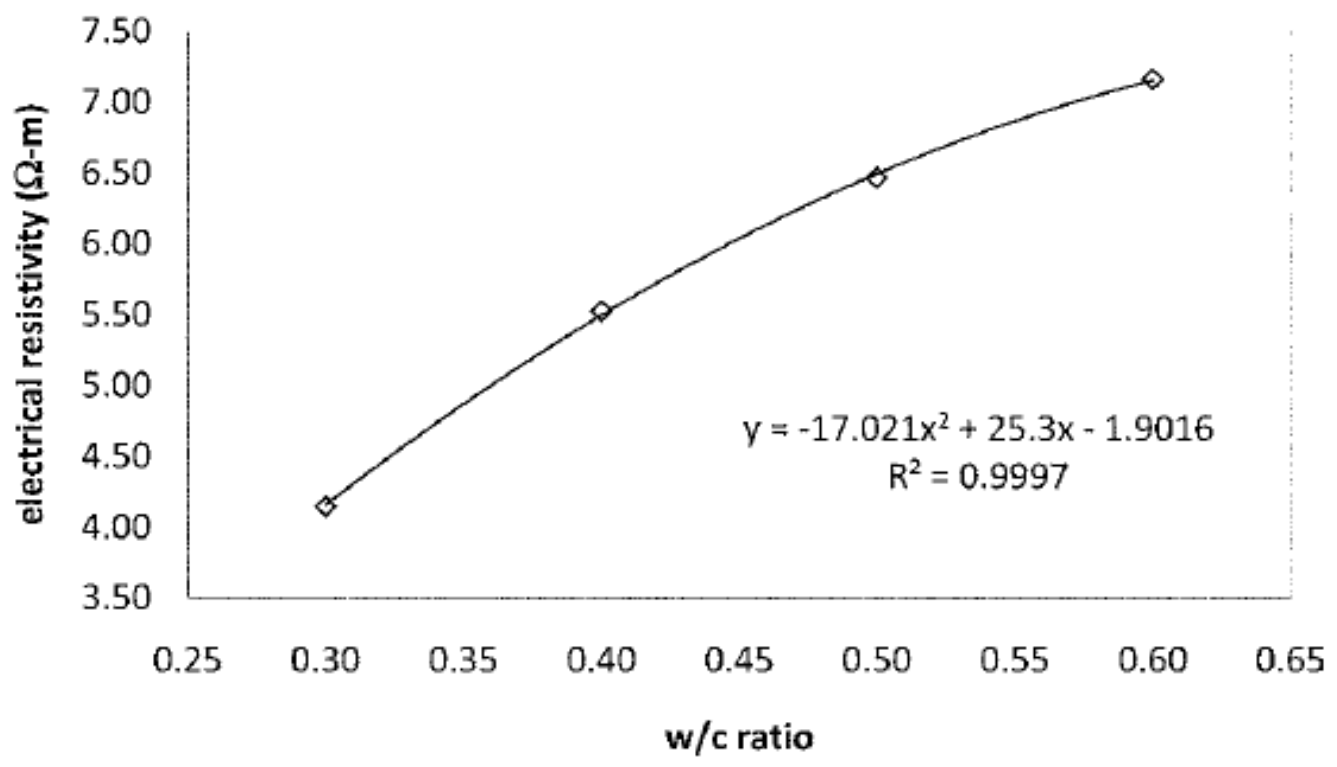

(b)

Figure 2.8. Electrical resistivity versus w/c of concrete: a) No fly ash; b) $25 \%$ fly ash [8].

Mancio et al. [8] conducted the electrical resistivity measurement test on concrete samples with various w/c ratios of $0.3,0.4,0.5$ and 0.6 with $0 \%$ and $25 \%$ of fly ash (Fig. 2.8). They monitored electrical resistivity of fresh concrete during the first 2 hours using the Wenner probe. Based on the results of their experiments, it was reported that the electrical resistivity of fresh concrete increased with increasing $\mathrm{w} / \mathrm{c}$ ratio. They suggested that at lower w/c ratios, the concentration of ions in pore solution was more and conductivity therefore increased which correlated to the lower electrical resistivity. However, this conclusion quite contradicts with data reported in the literature. The mix proportion of these samples presented in Table 2.3, indicated that in their experiments, the aggregate volume fraction also increased along with w/c ratio which most-likely is the governing effect on electrical resistivity rise not increase in $\mathrm{w} / \mathrm{c}$ ratio. 
Table 2.3. Mixture proportions of concrete samples [8].

\begin{tabular}{|c|c|c|c|c|c|c|c|c|}
\hline \multirow{2}{*}{$\begin{array}{c}\text { Mixture } \\
\text { no. }\end{array}$} & \multirow{2}{*}{$\begin{array}{c}\text { w/c } \\
\text { wly ash } \\
\text { replacement } \\
\text { ratio }\end{array}$} & \multicolumn{5}{|c|}{ Amounts $\left(\mathrm{kg} / \mathrm{m}^{3}\right)$} & \begin{tabular}{c} 
Aggregate \\
fraction \\
$\mathbf{V}_{\mathbf{a}}$ \\
\cline { 4 - 7 }
\end{tabular} \\
\cline { 5 - 8 } & & Cement & Fly ash & Water & FA & CA & $(\%)$ \\
\hline 1 & 0.30 & 0 & 722 & 0 & 217 & 557 & 858 & 54 \\
2 & 0.40 & 0 & 541 & 0 & 217 & 710 & 858 & 60 \\
3 & 0.50 & 0 & 433 & 0 & 217 & 802 & 858 & 64 \\
4 & 0.60 & 0 & 361 & 0 & 217 & 863 & 858 & 66 \\
\hline 5 & 0.30 & 0.25 & 541 & 180 & 217 & 557 & 858 & 54 \\
6 & 0.40 & 0.25 & 406 & 135 & 217 & 710 & 858 & 60 \\
7 & 0.50 & 0.25 & 325 & 108 & 217 & 802 & 858 & 63 \\
8 & 0.60 & 0.25 & 271 & 90 & 217 & 863 & 858 & 66 \\
\hline
\end{tabular}

Note: FA and CA are coarse and fine aggregates, respectively; and specific gravity of cement, fly ash, $\mathrm{FA}$, and $\mathrm{CA}$ in $\mathrm{g} / \mathrm{cm}^{3}$ are $3.15,2.60,2.68$, and 2.68 , respectively.

\subsection{Electrical resistivity measurement techniques for concrete, mortar and cement paste}

Conventionally, concrete was regarded as a conductor with resistance $(\Omega)$, and its resistance was measured by placing it in an electrical circuit between two electrodes that passed current from one to the other $[1,3,28,29]$. In this setup the potential drop is measured, and the concrete impedance is defined as the ratio of the potential drop to the applied current. Two main problems which add errors to this conventional measurement technique are polarization of the specimen (if D.C. current is applied) and capacitive reactance effect (if A.C. current is applied). Polarization occurs due to flowing current through an electrolyte that results in the establishment of an opposite potential to the 
applied potential at the electrodes. Concrete acts as a conductive electrolyte [30]; therefore, this phenomenon leads to the deviation of measured resistance from the actual resistance of concrete. On the other hand, because of the double-layer effect between electrode and electrolyte (concrete) [31] which acts as a capacitor, the measured impedance is resulted from both resistance and capacitive reactance of concrete [32]. Therefore, the determination of resistance component (i.e., the parameter of interest) becomes complicated.

In 1985, Hughes et al. developed a new method [31] of measuring the electrical resistivity of the concrete, mortar and cement paste, which eliminated some of the difficulties associated with the conventional D.C. measurement technique. The setup of this method is illustrated in Fig. 2.9. A constant-current generator passes a square wave form alternative current through the concrete, mortar or cement paste specimen and holds the amplitude of the current constant regardless of its resistance.

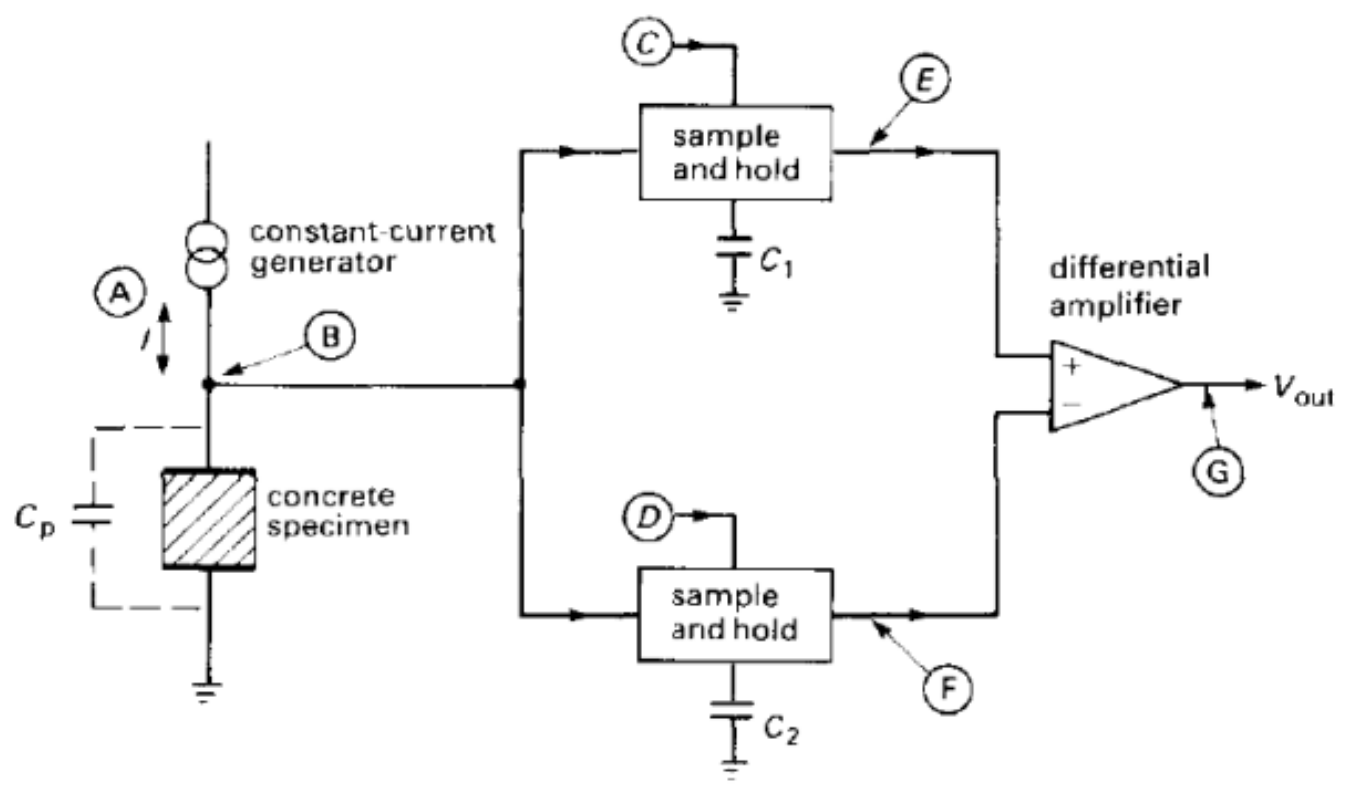

Figure 2.9. Block diagram of the electrolytic ohmmeter [31]. 
The term $\mathrm{C}_{\mathrm{p}}$ in Fig. 2.9 is indicative of the capacitive reactance of the specimen in practice which along with the resistance of concrete composes the voltage across the specimen. Capacitance slows down the rate of change in current when reversed and consequently the peak voltage is less than the peak voltage of pure resistance until it is fully charged again (see Fig. 2.10). Hence, exactly at the moment in which voltage reaches the maximum value, two sample-and-hold circuits $\mathrm{C}$ and $\mathrm{D}$ take samples from positive and negative input analogue voltage at $\mathrm{B}$, respectively. Both voltage values are recorded continuously by a differential amplifier. Furthermore, the A.C. current which is applied by constant-current generator has a very small amplitude of $0.5 \mathrm{~mA}$ and very short pulse time of $10 \mathrm{~ms}$ (constant frequency of $100 \mathrm{~Hz}$ ) which both minimize the electrical field generated by polarization; i.e., the smaller the current amplitude as well as the higher the pulse frequency, the less the polarization effect. After using this method on different samples and comparing them to conventional D.C. and A.C. electrical resistivity measurements, Hughes et al. [31] concluded that the polarization effect increased, whereas the capacitive reactance decreased the measured resistivity of concrete, mortar or cement paste. Furthermore, capacitance increased with time and increasing the w/c ratio. In addition, it was reported [31] that by increasing w/c ratio for constant cement content as well as increasing the cement content for constant $w / c$ ratio, the resistivity of concrete decreased. However, the decrease in resistivity of concrete for more cement content is most likely attributed to the less fraction of aggregates with quite larger resistivity values compared to that of cement paste which was not studied and considered. Also, the effect of magnitude of applied frequency on the capacitance effect was neglected. 


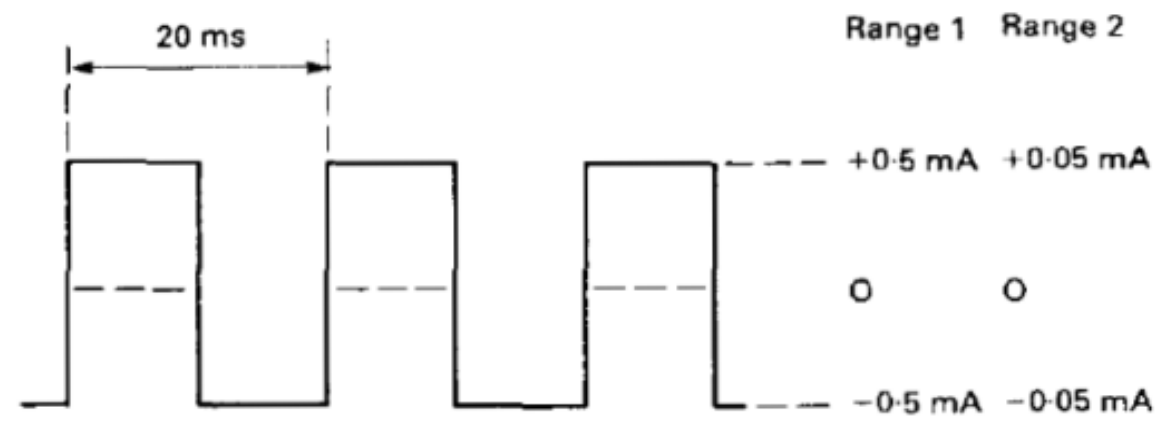

A: CONSTANT-CURRENT WAVE-FORM

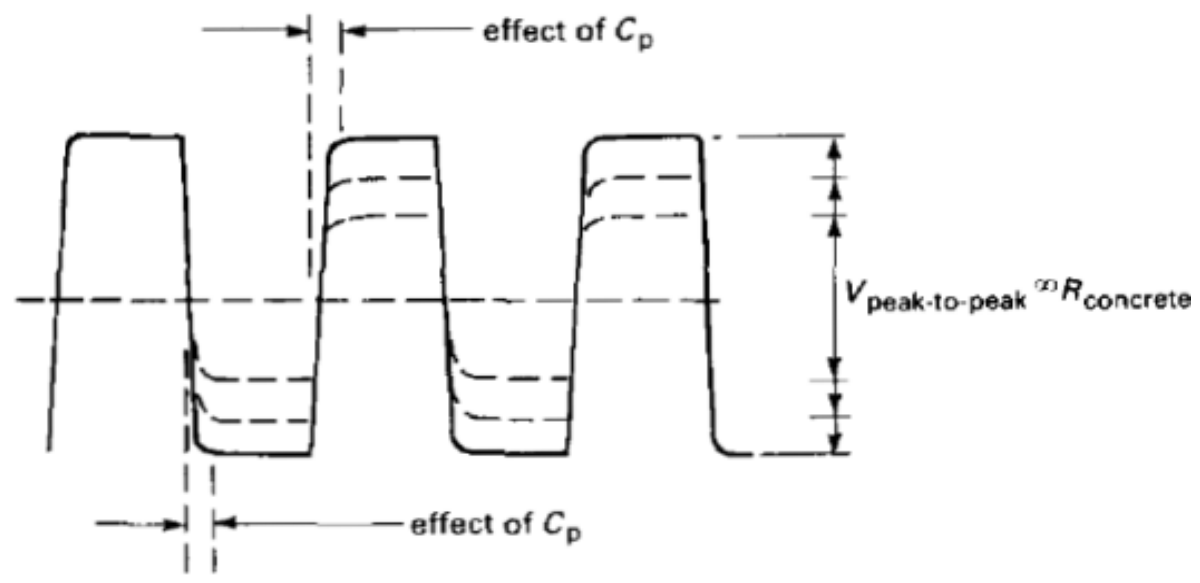

B: VOLTAGE ACROSS CONCRETE

Figure 2.10. Electrolytic ohmmeter mechanism: A) Applied current; B) Corresponding voltage across concrete [31].

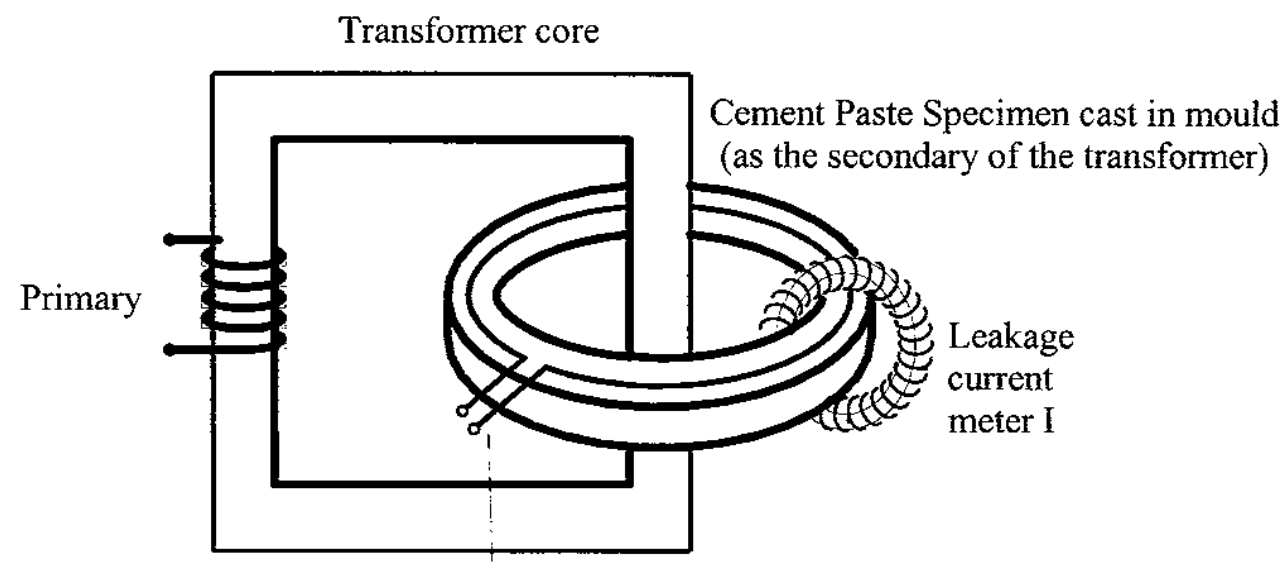

Toroidal volatage measurement $\mathrm{V}$

Figure 2.11. Schematic of non-contact resistivity measurement of cement paste [5]. 
In 2001, Li et al [4] proposed a new technique to measure the electrical resistivity of cement-based materials to eliminate two main errors associated with resistance measurement of concretes and cement pastes using electrodes. The new method eliminated the electrodes used in conventional testing for resistivity, and rather used a transformer as shown in Fig. 2.11. The output or secondary coil in transformer is substituted with a ring and rectangular sectional area of cement paste specimen and this way the current passes though material without any contact of electrodes. In 2008, Li et al. [15] used the same setup for non-contact electrical resistivity measurement; but on concrete samples with trapezoidal cross sections as shown in Fig. 2.12.
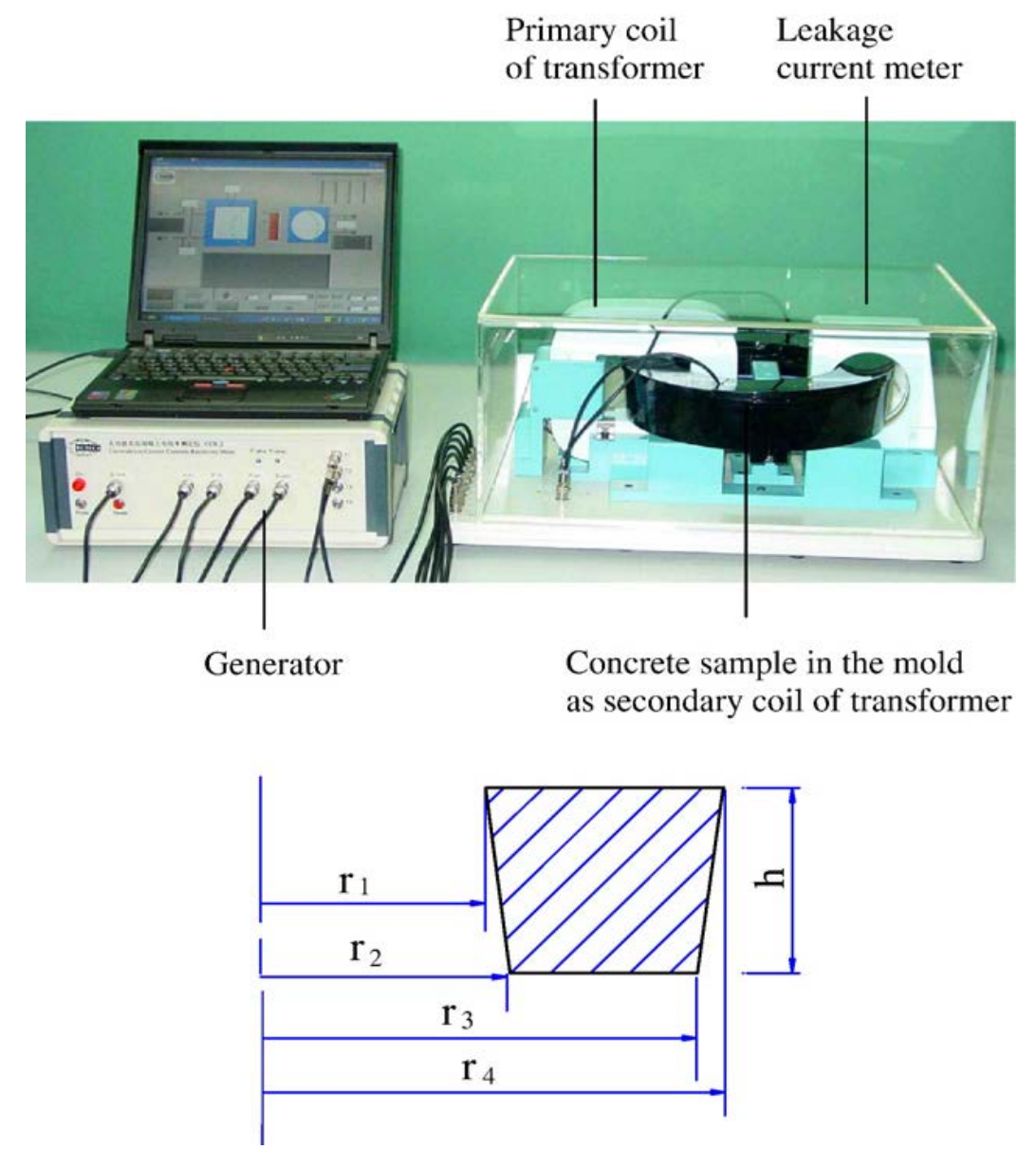

Figure 2.12. Non-contact electrical resistivity measurement instrument and mold cross section [15]. 
In this technique, the toroidal voltage inducted in concrete sample, $\mathrm{V}$, and corresponding toroidal current, I, are measured and the resistance of concrete are obtained based on Ohm's law. In order to derive the resistivity of concrete having the cross sectional area details given in Fig. 2.12, Li et al. used the following equation:

$$
\rho=\frac{h}{2 \pi}\left[-\frac{r_{1}}{r_{2}-r_{1}} \ln \frac{r_{2}}{r_{1}}+\ln \frac{r_{3}}{r_{2}}+\frac{r_{4}}{r_{4}-r_{3}} \ln \frac{r_{4}}{r_{3}}\right] \frac{V}{I}
$$

In 2010, Mancio et al. used a four-electrode Wenner probe for the electrical resistivity measurement of concrete specimens [8] (Fig. 2.13). This way, the instrument applies an AC current with $1 \mathrm{kHz}$ sinusoidal wave through the concrete sample when the probe is immersed in fresh concrete. If the applied current through outer electrodes connected to circuit is denoted by $\mathrm{I}_{0}$ and potential drop through concrete between inner electrodes is denoted by $\mathrm{V}_{\mathrm{c}}$ (Fig. 2.13), the resistance $\mathrm{R}_{\mathrm{c}}$ is given by Ohm's law. The electrical resistivity of concrete $\left(\rho_{c}\right)$ can then be derived by knowing the geometry factor k $(\mathrm{m})$ of the probe setup as:

$$
\rho_{C}=K R_{C}=K \frac{V_{C}}{I_{0}}
$$

where the geometry factor $(k)$ of probe as a function of electrodes spacing (a) is defined by:

$$
K=4 \pi a
$$




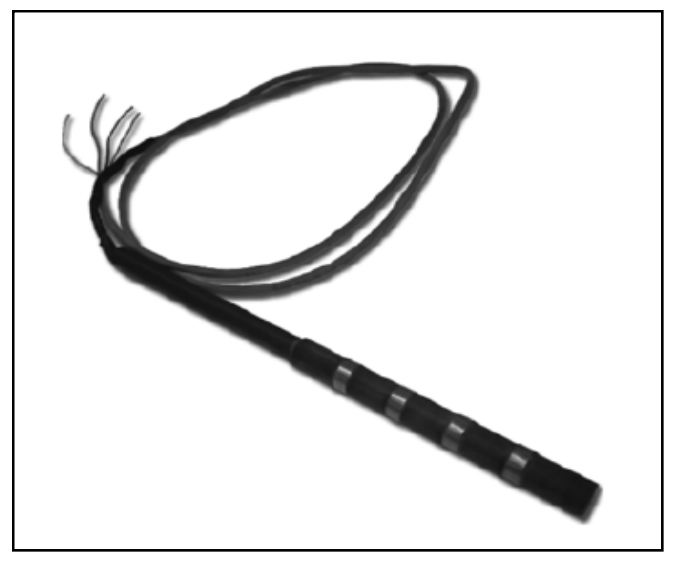

(a)

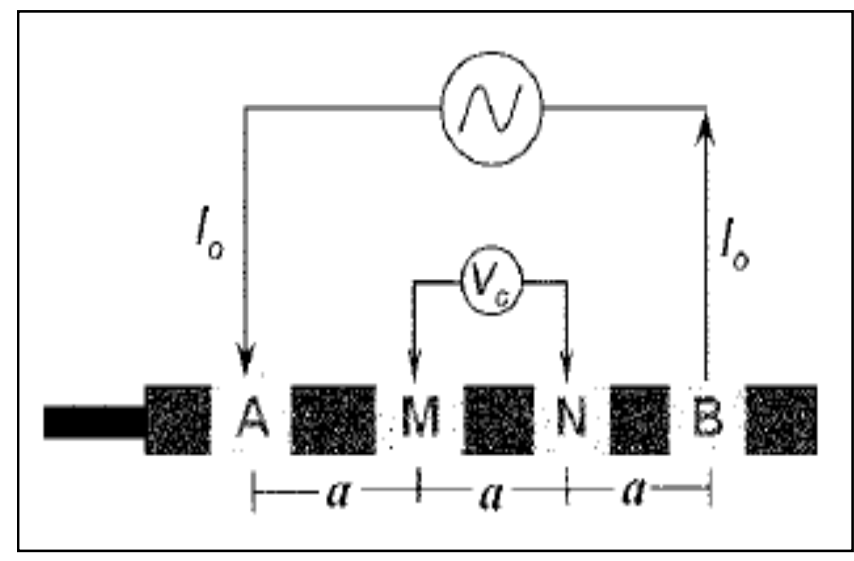

(b)

Figure 2.13. Electrical resistivity probe: a) actual probe; b) schematic shape [8].

\subsection{Effect of hydration or time on electrical resistivity}

The hydration process of cement-based materials has a complex mechanism. In concrete, it depends on various factors such as mixture proportions like w/c, physical and chemical properties of aggregates, chemical composition of cementitious materials and liquid phase, type of admixture, temperature, curing method (e.g. sealed curing, moist-curing). The cement paste is the most significant component in concrete that determines its fresh and hardened properties, and its characteristics are time dependent because of hydration process. As a result, in the study of the electrical resistivity of concrete especially in fresh state the effect of hydration time needs to be considered..

Electrical resistivity of concrete and cement paste increases as the hydration proceeds. This increase with time was reported by Whittington et al. [16] specifically after setting; however, they observed a decrease during first 5 hours (close to final setting time) of cement paste samples. They suggested that the resistivity decrease was either because of 
heat release and temperature increase as a result of chemical reactions, or increase in concentration of ions in pore solution which decreased pore solution resistivity. Thus, resistivity measurement can be used as a potential method to estimate the degree of hydration in concrete.

Based on resistivity-time curves for different w/c pastes, as shown in Fig. 2.3, Li et al. [5] suggested four stages of hydration: dissolving period (I), a competition period (II), a setting (III) and hardening period (IV) . These different stages were defined by indicating specific points as their boundaries on $\rho$-t curves (Fig. 2.3). M ( $(\mathrm{m}), \rho(\mathrm{m})$ ) which represents the minimum critical point; $\mathrm{L}(\mathrm{t}(\mathrm{l}), \rho(1))$ indicating the point in time at which almost level (plateau) curve ends and resistivity starts to rise markedly and I (t(i), $\rho(\mathrm{i})$ ) showing the point of inflection at which concavity changes from upward to downward; i.e., second derivative of resistivity with respect to time equals zero.

In 2006, Li et al., using the electrical resistivity development curve (Fig. 2.2-a) as well as corresponding rate of electrical resistivity (Fig. 2.2-b) showed that four different periods can be described as follows [14]:

1. Dissolution of ions (Period I) in pore solution because of chemical reactions between cement particles and water is dominant and consequently conductivity increases and correlates to decrease in resistivity up to initial setting time $\left(\mathrm{t}_{\mathrm{m}}\right)$.

2. Setting period (II) starts at initial setting time, $\mathrm{t}_{\mathrm{m}}$, in which the resistivity of the paste is minimum and after this point it increases slowly because of the formation of Ettringite, $\mathrm{CH}$ and $\mathrm{CSH}$ up to the point that suddenly the rate of increase in 
resistivity $(\mathrm{d} \rho / \mathrm{dt})$ considerably grows, $\mathrm{t}_{\mathrm{a}}$, that is indicative of the final setting time and beginning of hardening.

3. Acceleration period (III) occurs after $t_{a}$ in which the rate of change in electrical resistivity $(\mathrm{d} \rho / \mathrm{dt})$ is rapidly ascending. At the end of this period the rate reaches its peak, $t_{i}$, which is corresponding to the inflection point on resistivity-time curve.

4. Deceleration period (IV) starts from $t_{i}$ and although the growth in resistivity continues, $(\mathrm{d} \rho / \mathrm{dt})>0$, the rate of change $(\mathrm{d} \rho / \mathrm{dt})$ drops after this point. The chemical reaction is converted to diffusion control reaction at transitional point $\mathrm{t}_{\mathrm{i}}$.

Li et al. [15] proposed a model to estimate the hydration degree $\alpha(t)$ of concrete by measuring the bulk resistivity $\rho(t)$ and extracted pore solution resistivity $\rho_{0}(t)$ during first 48 hours of concrete age. The estimated values from the model were close to the experimental results for porosity using Mercury Intrusion Porosimetry (MIP) and degree of hydration based on Thermo-gravimetric analysis (TGA) conducted on dehydrated samples. The degree of hydration in TGA is defined as the fraction of chemically bonded water content at time $t$ to that of completely hydrated cementitious materials. Considering ignition loss of cement $\left(\mathrm{L}_{\mathrm{C}}\right)$ and fly ash $\left(\mathrm{L}_{\mathrm{FA}}\right)$ and knowing the ratio of fly ash to the entire cemnetitious materials $(\beta)$, the degree of hydration $\alpha(t)$ is expressed as via:

$$
\alpha(t)=\left[\frac{W_{105}}{W_{950}}\left(1-(1-\beta) L_{C}-\beta L_{F A}\right)-1\right] / \frac{W_{n}}{(C+F A)_{\text {comp }}}
$$


where $\alpha(t)$ is the degree of hydration at time $t ; W_{105}$ and $W_{950}$ are sample weights at temperature $105{ }^{\circ} \mathrm{C}$ and $950{ }^{\circ} \mathrm{C}$; and $\frac{W_{n}}{(C+F A)_{c o m p} \text {. }}$ is the chemical bonded water per gram completely hydrated cementitious material. The amount of chemically bound water for completely hydrated cement can be determined based on Bogues's equation [33]. The typical value of 0.23 for OPC was suggested by Taylor [34]. For fly ash incorporated cementitious material, this value was reported to be slightly less because of the dual effects of fly ash particles in the mixture; i.e., pozzolanic reaction and filler function [35].

Li et al. [15] also established the $\rho(t)-t$ and $d \rho(t) / d t-t$ curves for three different concrete mixtures with w/c ratio of 0.4 including $0 \%, 25 \%$ and $50 \%$ of class $\mathrm{F}$ fly ash which are shown by C0.4, C0.4FA25 and C0.4FA50, respectively, in Fig. 2.14. They observed that the similar hydration behavior found for cement pastes [5] is also valid for concrete samples; i.e., five stages of dissolution, competition of dissolution-precipitation, setting, hardening and hardening deceleration periods.

Li et al. [14] proposed a model (Eq. 2.9) to find the porosity $\varphi(t)$ of two-component system using Archie's law [19]:

$$
\begin{aligned}
& \frac{\rho(t)}{\rho_{0}(t)}=\varphi(t)^{-m(t)} \\
& m(t)=m_{1}+\frac{\ln \rho(t)-\ln \rho_{1}}{\ln \rho_{2}-\ln \rho_{1}}\left(m_{2}-m_{1}\right)
\end{aligned}
$$


(a)
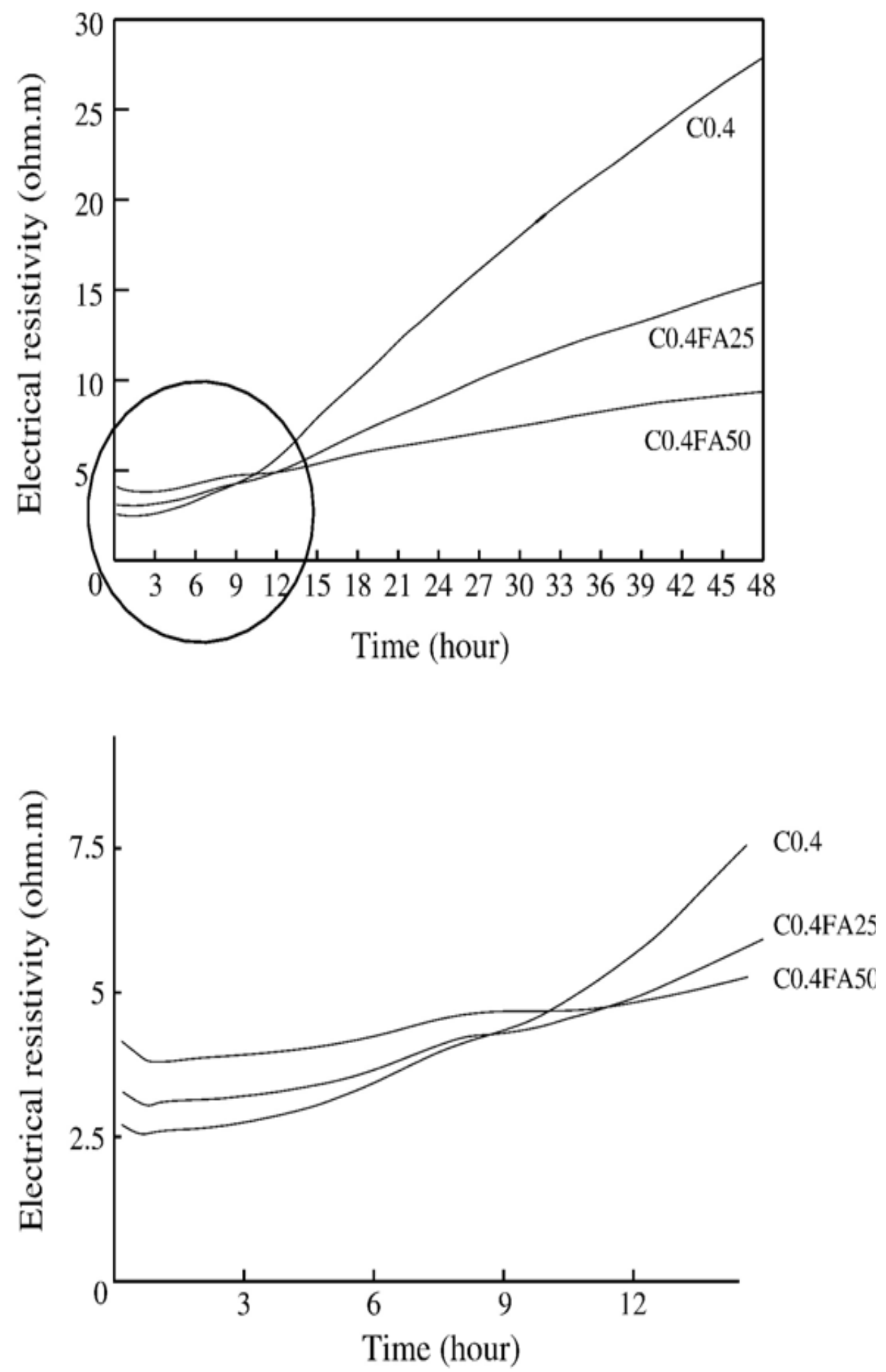
(b)
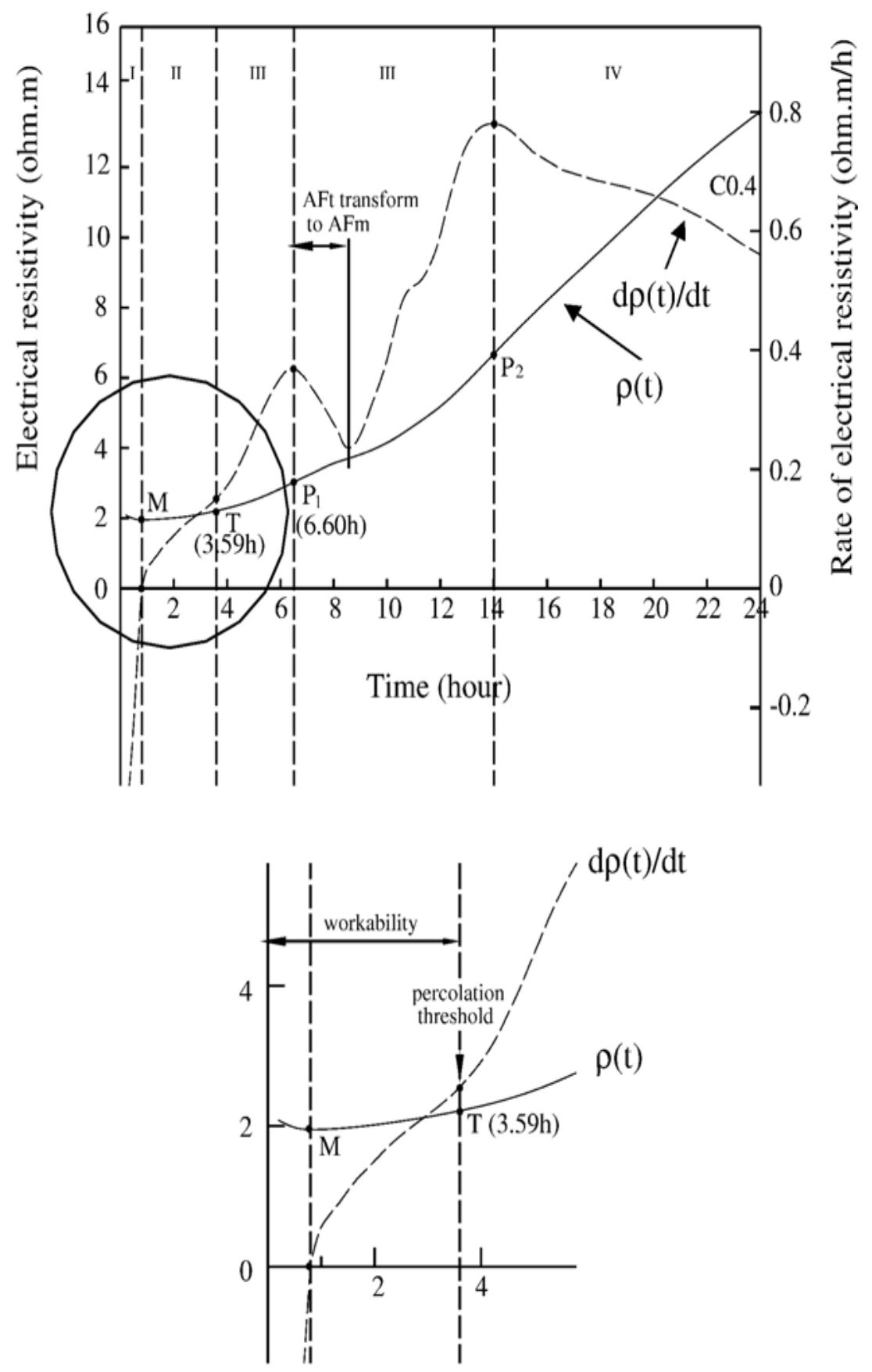

Detail

Figure 2.14. Bulk electrical resistivity and resistivity rate of fresh concrete with the w/c of 0.40: (a) Electrical resistivity development $\rho(t)-t$; (b) Rate of resistivity development $\mathrm{d} \rho(\mathrm{t}) / \mathrm{dt}-\mathrm{t}[15]$. 
where $\rho_{1}$ and $\rho_{2}$ are the bulk resistivity whereas $m_{1}$ and $m_{2}$ are $m$ exponent values at initial recording point and at the age of 24 hours, respectively. Accordingly, calculating m from Eq. 2.10 and substituting it into Eq. 2.9 along with the measured bulk and pore solution resistivity at a specific time, corresponding porosity $\varphi(t)$ at that time can be determined [15].

Powers and Brownyard [33] proposed a model to characterize the hydration process in paste which was assumed to have three components including unhydrated cement, hydration product and capillary pores that were considered as main conductive paths at early age. Li et al. [15] ignoring the hydration of class F fly ash at early age proposed the following model to correlate the degree of hydration to porosity:

$\alpha(t)=\left[\frac{w / c}{D_{w}}-V_{\text {Total }} . \varphi(t)\right] \cdot \frac{D_{c} D_{h}}{D_{c}-D_{h}}$

where $w / c$ is the water to cementitious materials ratio; $V_{\text {Total }}$ is the total volume of each constituent in the concrete; and $\mathrm{D}_{\mathrm{w}}, \mathrm{D}_{\mathrm{c}}$ and $\mathrm{D}_{\mathrm{h}}$ represent the density of pore solution, cement and hydrates, respectively. Assuming $D_{\mathrm{w}}=1.01 \mathrm{~g} / \mathrm{cm}^{3}, D_{\mathrm{c}}=3.15 \mathrm{~g} / \mathrm{cm}^{3}, D_{h}=1.529$ $\mathrm{g} / \mathrm{cm}^{3}$ [33] and $\mathrm{w} / \mathrm{c}=0.4$ for the mixtures studied by Li et al. [15], they simplified the model in Eq. 2.11 to define the degree of hydration $\alpha(t)$ as a function of porosity $\varphi(t)$.

$\alpha(t)=2.971\left[0.4-V_{\text {Total }} . \varphi(t)\right]$

The variation of degree of hydration derived from this model was plotted in Fig. 2.15. The estimated data were reasonably close to the results of dehydration conducted using thermo-gravimetric analysis. 


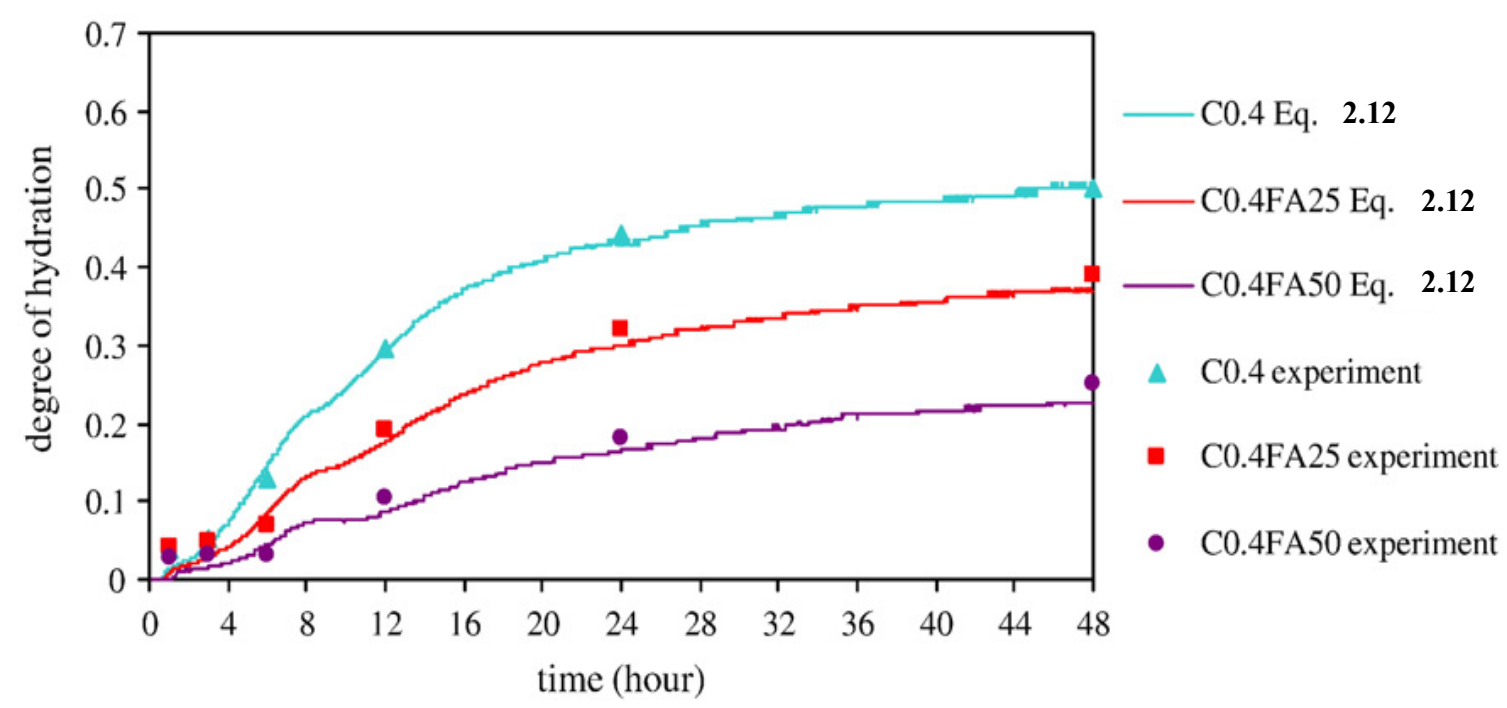

Figure 2.15. Degree of hydration with time during first $48 \mathrm{hr}$ for concrete samples [15]. (Solid curves are calculated from Eq. 2.12, whereas the points show the experimental results).

Mancio et al. [8], based on the statistical analysis of their results presented in Table 2.4, suggested that the effect of time on the electrical resistivity of the concrete samples was insignificant during the first two hours after mixing; i.e., the change in electrical resistivity of fresh concrete during the first 2 hours was negligible.

Table 2.4. Electrical resistivity of concrete samples as a function of time and $\mathrm{w} / \mathrm{c}$ [8].

\begin{tabular}{c|c|c|c|c}
\hline \multirow{2}{*}{ Time, minutes } & \multicolumn{4}{|c}{ Resistivity $(\Omega$-m) for different mixtures } \\
\cline { 2 - 5 } & 0.30 & 0.40 & 0.50 & 0.60 \\
\hline 0 & 2.22 & 2.91 & 3.39 & 3.98 \\
\hline 10 & 2.19 & 3.25 & 3.59 & 4.12 \\
\hline 20 & 2.27 & 3.01 & 3.60 & 4.05 \\
\hline 30 & 2.20 & 3.08 & 3.52 & 4.03 \\
\hline 40 & 2.10 & 2.91 & 3.68 & 3.83 \\
\hline 50 & 2.06 & 2.96 & 3.68 & 3.85 \\
\hline 60 & 2.15 & 2.95 & 3.61 & 3.79 \\
\hline 70 & 2.08 & 2.92 & 3.51 & 3.61 \\
\hline 80 & 2.19 & 2.92 & 3.47 & 3.68 \\
\hline 90 & 2.33 & 2.81 & 3.50 & 3.74 \\
\hline 100 & 2.34 & 2.87 & 3.41 & 3.79 \\
\hline 110 & 2.27 & 2.97 & 3.35 & 3.64 \\
\hline 120 & 2.21 & 2.84 & 3.36 & 3.63 \\
\hline
\end{tabular}


Salem [9] also monitored the conductance $(\mathrm{mS})$ of the OPC pastes containing silica fume during first 24 hours. As shown in Fig. 2.16, the Conductance-Time curves were reported to reveal two peeks, the first one was reported to be attributed to the beginning of OPC hydration and, the second peek on the other hand was attributed to the ettringitemonosulfate transformation.

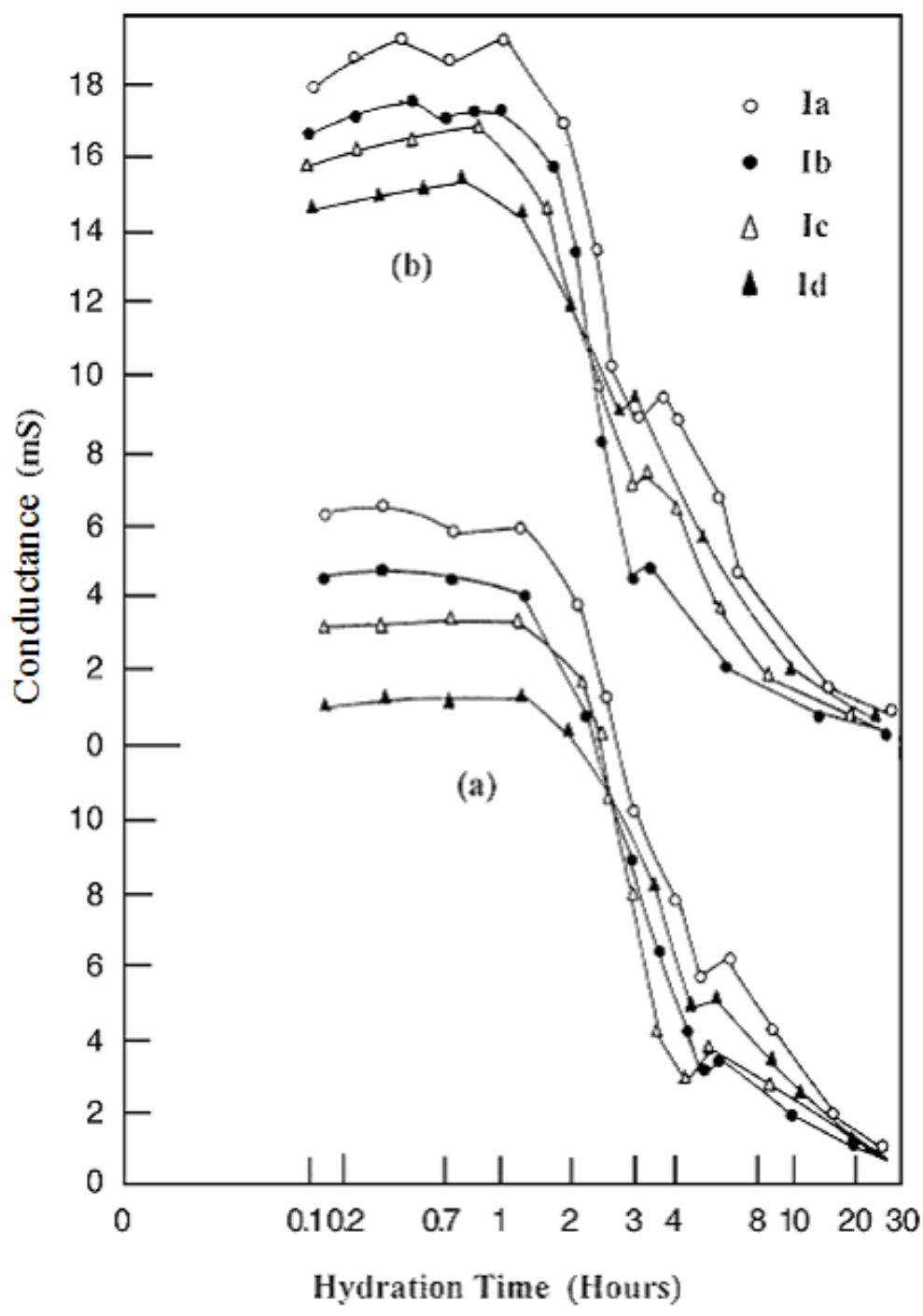

Figure 2.16. Conductance-Time curves of OPC plus silica fume paste mixtures. Ia, Ib, Ic and Id represent 10, 20, 30 and 50 percent silica fume replacement, respectively; for w/c of 0.55 at two temperatures: (a) $25^{\circ} \mathrm{C}$; and (b) $45^{\circ} \mathrm{C}$ [9]. 


\subsection{Pore solution (liquid phase) resistivity}

Cement paste matrix which can be regarded as conductive component of concrete compared to nonconductive aggregates, dominantly determines the electrical properties of the concrete. Nikkannen [30] reported that electrolytic conduction through cement paste controls resistivity of concrete and experimental data obtained by Monfore and Hammond and Robson $[1,3]$ verified this claim and suggested that ionic species such as $\mathrm{OH}^{-}, \mathrm{K}^{+}, \mathrm{Na}^{+}, \mathrm{Ca}^{2+}$ and $\mathrm{SO}_{4}{ }^{2-}$ in pore solution transport the electrical charge through cement paste.

On the other hand, the electrical resistivity of pore solution plays a significant role on the resistivity of paste matrix. Whittington et al. [16] supported this idea that pore solution determines the electrical resistivity of cement paste. However, they found it pretty speculative to sub-divide the cement paste to solution phase (conductive component) and solid phase (cement particles) at any specific hydration age because of constant change in the amount of solution phase and also the change in concentration of ions during the hydration process; i.e., the pore solution amount and associated ionic concentration are both time dependent.

Li et al. [5] reported that from the alkali sulfates and cement particles, $\mathrm{K}^{+}$and $\mathrm{Na}^{+}$and $\mathrm{SO}_{4}{ }^{2-}$ were dissolved into liquid immediately after mixing cement with water. The $\mathrm{Ca}^{2+}$ and $\mathrm{OH}^{-}, \mathrm{Al}$ and $\mathrm{Si}$ ions were released into liquid phase due to dissolution of free lime $(\mathrm{CaO}), \mathrm{C}_{3} \mathrm{~S}$ and $\mathrm{C}_{2} \mathrm{~S}$. However, the concentrations of the $\mathrm{OH}^{-}, \mathrm{K}^{+}, \mathrm{Na}^{+}, \mathrm{Ca}^{2+}$ and $\mathrm{SO}_{4}{ }^{2-}$ were dominant in total pore solution conductivity [36]. 
In another study, using a non-contact electrical resistivity measurement, Li et al. [26] monitored the resistivity development of cement pastes incorporated with a set retarder of different dosages $0 \%, 0.1 \%, 0.15 \%$ and $0.2 \%$ (by weight with respect to the solid content) at the w/c ratio of 0.30 during first 24 hours (see Fig. 2.5). They measured resistivity of the extracted pore solutions $\left(\rho_{0}(t)\right)$ of retarder incorporated paste samples at minimum point on bulk resistivity-time $\left(\mathrm{t}_{\mathrm{m}}\right)$ curve (Table 2.5$)$. They concluded that with increase of retarder dosage, the ion concentration in pore solution increases and corresponding resistivity decreases at fresh state. However, this decrease in resistivity is most likely because of longer time of pore solutions extraction $(16,51,81$ and 178 minutes) and more ions release in pastes with higher portion of retarders, which increases the conductivity, not the effect of greater dosage of retarders.

Table 2.5. Electrical resistivity of liquid phase $\rho_{0}\left(t_{m}\right)$ at minimum point time of pastes with w/c of 0.3 [26].

\begin{tabular}{|ccc|}
\hline Retarder dosage & $\boldsymbol{\rho}_{\mathbf{0}}\left(\mathbf{t}_{\mathbf{m}}\right)$ & $\begin{array}{c}\text { Extracting time at } \mathbf{t}_{\mathbf{m}} \\
(\%)\end{array}$ \\
$(\Omega . \mathrm{m})$ & $(\mathrm{min})$ \\
\hline 0.00 & 0.252 & 16 \\
0.10 & 0.245 & 51 \\
0.15 & 0.235 & 81 \\
0.20 & 0.229 & 178 \\
\hline
\end{tabular}


Although the electrical resistivity of pore solution in a cement paste is dependent on ions concentration such as $\mathrm{OH}^{-}, \mathrm{K}^{+}, \mathrm{Na}^{+}, \mathrm{Ca}^{2+}, \mathrm{Cl}^{-}$and $\mathrm{SO}_{4}{ }^{2-}$, Snyder et al. [12] proposed that by considering only concentrations of $\mathrm{OH}^{-}, \mathrm{K}^{+}$and $\mathrm{Na}^{+}$as governing ionic species [37, 38], pore solution conductivity can be accurately predicted. The contribution of each ion to the total conductivity of pore solution differs from one to the other. Snyder et al. [12] showed that the contribution of each ion is a function of ion molar concentration $\left(\mathrm{C}_{\mathrm{i}}\right)$ and the ionic strength $\left(\mathrm{I}_{\mathrm{M}}\right)$ which also depends on ion concentration.

Equivalent conductivity is defined as the electrical conductivity per ionic concentration and denoted by $\lambda\left(\mathrm{Scm}^{2} / \mathrm{mol}\right)$. The electrical conductivity of pore solution can be expressed as weighted sum of equivalent conductivity for each ionic species such as $\mathrm{OH}^{-}$:

$$
\sigma=\sum z_{i} C_{i} \lambda_{i}
$$

where $\mathrm{z}_{\mathrm{i}}$ and $\mathrm{C}_{\mathrm{i}}$ are the species valence and molar concentration, respectively. Although Eq. 2.13 shows a linear relationship between conductivity and molar concentration initially, since the equivalent conductivity itself is a function of ionic concentration, this relationship would not be linear anymore as discussed in the literature [39].

Snyder et al. [12] suggested that for each ionic species, the equivalent conductivity $\lambda_{\mathrm{i}}$ is a portion of ultimate equivalent conductivity $\lambda_{i}^{\circ}$ corresponding to the infinite dilution (C $\rightarrow 0)$ as follows:

$$
\lambda_{i}=\frac{\lambda^{0}}{1+G_{i} I_{m}{ }^{1 / 2}}
$$


$I_{m}=\frac{1}{2} \sum z_{i}^{2} C_{i}$

where $I_{m}$ is the ionic strength in $\mathrm{mol} / \mathrm{l}$; and $G_{i}$ is the empirical coefficient in $(\mathrm{mol} / \mathrm{l})^{-1 / 2}$ obtained from experiments at specific temperature.

Considering Eqs. 2.13 to 2.15 , it is concluded that the pore solution conductivity is directly proportional to square root of ion concentration (i.e., $\sigma \propto \sqrt{c}$ ). Therefore, we can approximate conductivity of a pore solution before dilution $\left(\sigma_{1}\right)$ if we have the conductivity of pore solution after dilution $\left(\sigma_{2}\right)$ via:

$\frac{\sigma_{1}}{\sigma_{2}}=\sqrt{\frac{C_{1}}{C_{2}}}$

where $\mathrm{C}_{1}$ and $\mathrm{C}_{2}$ are the concentrations of ions in pore solution before and after dilution, respectively.

Li et al. [14] measured electrical resistivity of the pore solution extracted from cement pastes with w/c of $0.30,0.35$ and 0.40 which were denoted by S-P0.3, S-P0.35 and SP0.4, respectively (shown in Fig. 2.17). They concluded that resistivity of pore solution is linearly proportional to w/c ratio (Eq. 2.17); i.e., the lower w/c ratio results in lower pore solution resistivity. The higher pore solution conductivity in lower w/c pastes is most likely attributed to higher amount of ions per unit volume of pore solution. 


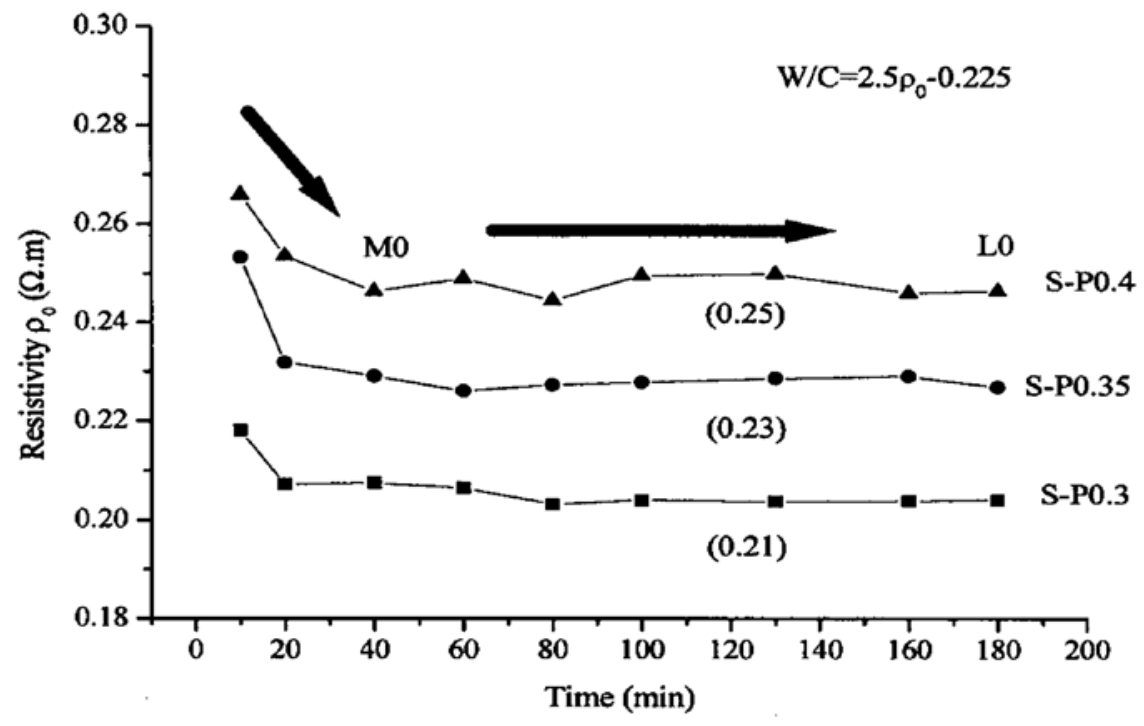

Figure 2.17. Electrical resistivity of pore solution $\left(\rho_{o}\right)$ in cement paste samples with various $\mathrm{w} / \mathrm{c}$ ratios [14].

$\frac{w}{c}=2.5 \rho_{o}-0.225$

\subsection{Temperature effect on electrical resistivity}

When the resistivity measurement is used to describe the characteristics of fresh or hardened concrete such as setting time, compressive strength and durability, the accuracy of such measurement should be verified in order to have valid results to draw any conclusion. Effect of temperature on resistivity and subsequently normalization to a reference temperature have been of a great importance to researchers since either in the lab or field, the temperature at which the resistivity is measured varies due to ambient or curing temperature and seasonal change respectively. Temperature influences concrete resistivity by changing the ion mobility, ion-ion and ion-solid interaction, as well as ion 
concentration in pore solution. Resistivity of concrete was reported to be inversely proportional to temperature $[40,41]$; i.e., it decreases as temperature increases.

A linear relationship in electrolytic solutions was suggested $[42,43]$ to be applicable for determining the resistivity of concrete at reference temperature $\theta$ (Eq. 2.18). However, this equation many years later was claimed to be only applicable over a low range of temperature variation around $\pm 5{ }^{\circ} \mathrm{C}$ to the reference temperature [42].

$\rho_{\theta}=(1+\alpha(T-\theta)) \rho$

where $\rho_{\mathrm{t}}$ and $\rho_{\theta}$ are resistivities of concrete at $\mathrm{T}{ }^{\circ} \mathrm{C}$ and $\theta{ }^{\circ} \mathrm{C}$, respectively; $\alpha\left({ }^{\circ} \mathrm{C}^{-1}\right)$ is the temperature coefficient of resistivity and was observed to be in $10^{-2}$ order (e.g. 0.021 ) $[42,43]$.

Resistivity-Temperature relationship can also be expressed using Hinrichson-Rusch Law which is generally applicable for most refractory materials $[16,44]$ via:

$\rho_{1}=\rho_{2} e^{a\left(\frac{1}{T_{1}}-\frac{1}{T_{2}}\right)}$

where $\rho_{1}$ and $\rho_{2}$ are resistivities of the concrete at temperature $T_{1}$ and $T_{2}$ in ${ }^{\circ} \mathrm{K}$; and a is the experimental constant in ${ }^{\circ} \mathrm{K}$.

Whittington et al. [16], comparing experimental data and calculated data by proposed equations, concluded that the inverse relationship between ambient temperature and resistivity of concrete, mortar or cement paste can be estimated with high precision using either of Eq. 2.18 or 2.19 . 
Arrhenius law which was proposed to relate the activation energy $\mathrm{E}_{\mathrm{a}}$ and temperature $\mathrm{T}$ to the rate of reaction in chemistry [45], has been also used by many researchers [46-48] as a popular method to describe the relationship between resistivity and temperature as follows:

$\rho_{T}=\rho_{0} e^{\left[\frac{E_{a, \rho}}{R}\left(\frac{1}{T}-\frac{1}{T_{0}}\right)\right]}$

where $\rho_{\mathrm{T}}$ and $\rho_{0}$ are resistivity values measured at temperature $\mathrm{T}\left({ }^{\circ} \mathrm{K}\right)$ and reference $\mathrm{T}_{0}$ $\left({ }^{\circ} \mathrm{K}\right)$, respectively; $\mathrm{R}$ is gas constant $\left(8.314 \mathrm{~J} / \mathrm{mol} .{ }^{\circ} \mathrm{K}\right)$; and $\mathrm{E}_{\mathrm{a}, \rho}$ is the activation energy for resistivity $(\mathrm{J} / \mathrm{mol})$.

Activation energy $\mathrm{E}_{\mathrm{a}, \rho}$ here is defined as amount of energy required to promote one mole of the ions such as $\mathrm{OH}^{-}, \mathrm{K}^{+}$and $\mathrm{Na}^{+}$in pore solution of concrete from equilibrium state to activated state to carry current flow under an electric field [49]. Activation energy is a parameter that reflects the temperature sensitivity of concrete resistivity; i.e., the larger the $\mathrm{E}_{\mathrm{a}, \rho}$ is, the more sensitive to temperature the resistivity of the concrete would be.

Chrisp et al. [42] reported that the values of the $E_{a, \rho}$ ranges from 16.9 to $42.8 \mathrm{~kJ} / \mathrm{mol}$ in hardened concrete which are most likely different from fresh concrete. They showed that decreasing concrete's saturation degree increases activation energy. Furthermore, use of pozzolanic materials was demonstrated by McCarter et al. [47] to increase the activation energy compared to mixtures only containing OPC.

Salem et al. [10] conducted the electrical conductivity measurement of the pastes during first 24 hours of hydration age at $30^{\circ} \mathrm{C}$ and $50^{\circ} \mathrm{C}$. They found that electrical conductivity 
of pastes at higher temperatures is always greater than that of lower temperatures which is attributed to acceleration effect of hydration reactions of cementitious materials.

Yanbo et al. [7] did an extensive investigation testing 200 concrete cylinders from 54 mixtures to study temperature effect on resistivity. They conducted a Dynamic Temperature Test (DDT) from $10{ }^{\circ} \mathrm{C}$ to $45^{\circ} \mathrm{C}$ and measured the corresponding resistivity at complete hydration (after 6 years) using a four point (Wenner) method. Some specimens were tested with $85 \%$ and $92 \%$ saturation degree (unsaturated) along with saturated specimens to take into account the effect of moisture content. As shown in Fig. 2.18 for a concrete sample with $\mathrm{w} / \mathrm{c}$ of 0.4 , they observed that resistivity of concrete decreases with increasing temperature. They also found that for samples cured in lower saturation degree, resistivity of concrete is higher which was in agreement with literature $[50,51]$; i.e., the higher the saturation degree is, the lower the electrical resistivity of concrete becomes.

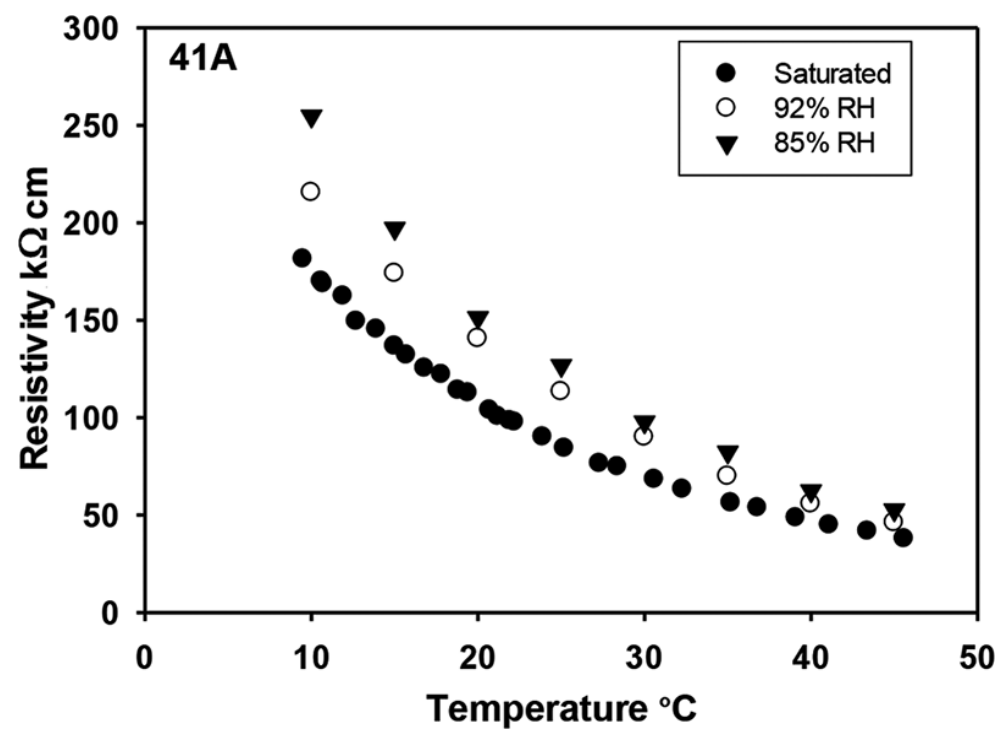

Figure 2.18. Resistivity variation with temperature for a concrete sample with w/c of 0.4 (labelled by $41 \mathrm{~A})$ in saturated and unsaturated ( $85 \%$ and $92 \%$ relative humidity) curing conditions after 6 years [7]. 
Table 2.6. Equations suggested for $\mathrm{E}_{\mathrm{a}, \rho}$ and $\rho_{21}$ correlation based on the type of concrete mixture [7].

\begin{tabular}{|c|c|}
\hline Equation & Concrete Mixture Properties \\
\hline Eq. 2.22 & Concrete with $\geq 20 \%$ fly ash \\
& Concrete with $>50 \%$ slag \\
\hline & Ordinary Portland cement (OPC) concrete \\
Eq. 2.23 & High alkalinity concrete (HA) \\
& Concrete with $<20 \%$ fly ash \\
& Concrete with $\leq 50 \%$ slag \\
\hline
\end{tabular}

Doing the regression analysis on Eq. 2.21 (Arrhenius law), parameters $\mathrm{E}_{\mathrm{a}, \mathrm{\rho}}$ and A were determined for all the concrete mixtures.

$\rho=A e^{\left[\frac{E_{a, \rho}}{R .(T+273.15)}\right]}$

where $\mathrm{A}$ is the resistivity when temperature $\mathrm{T}\left({ }^{\circ} \mathrm{C}\right)$ approaches infinity; i.e., $T \rightarrow \infty$. From the obtained activation energy for each particular mixture, they also concluded that if resistivity at a reference temperature such as $21{ }^{\circ} \mathrm{C}\left(\rho_{21}\right)$ is higher, the associated $\mathrm{E}_{\mathrm{a}, \rho}$ also would be higher than that of a lower resistivity. Accordingly, by regression analysis, Eq. 2.22 and Eq. 2.23 were proposed [7] for two general mixtures whose properties are presented in Table 2.6.

$$
\begin{aligned}
& E_{a, \rho}=3.7738 \ln \left(\rho_{21}\right)+9.7518 \\
& E_{a, \rho}=6.0157 \ln \left(\rho_{21}\right)+4.3121
\end{aligned}
$$

where activation energy is in $(\mathrm{kJ} / \mathrm{mol})$ and $\rho_{21}$ is in $\mathrm{k} \Omega . \mathrm{cm}$. 
In addition, using Eq. 2.22 and 2.23, Yanbo et al. [7] calculated resistivity percentage change per ${ }^{\circ} \mathrm{C}$ for specimens with different $\rho_{21}$. The results illustrated that for all of the concrete mixtures the resistivity change per ${ }^{\circ} \mathrm{C}$ is larger at a lower temperature than at a higher temperature as shown in Fig. 2.19.

Knowing the alkalinity of cement and percentage of supplementary cementitious materials (see Table 2.6), Yanbo et al.'s equations presented above can be used to normalize concrete resistivity to a reference temperature regardless of other mixture properties such as $\mathrm{w} / \mathrm{c}$ ratio and amount of aggregates. The activation energy used in the equations should be first determined based on $\rho_{21}$ which reflects the w/c ratio and aggregates effect itself; i.e., the smaller the $w / c$ ratio, the larger the $\rho_{21}$ and hence, higher $E_{a, \rho}$.

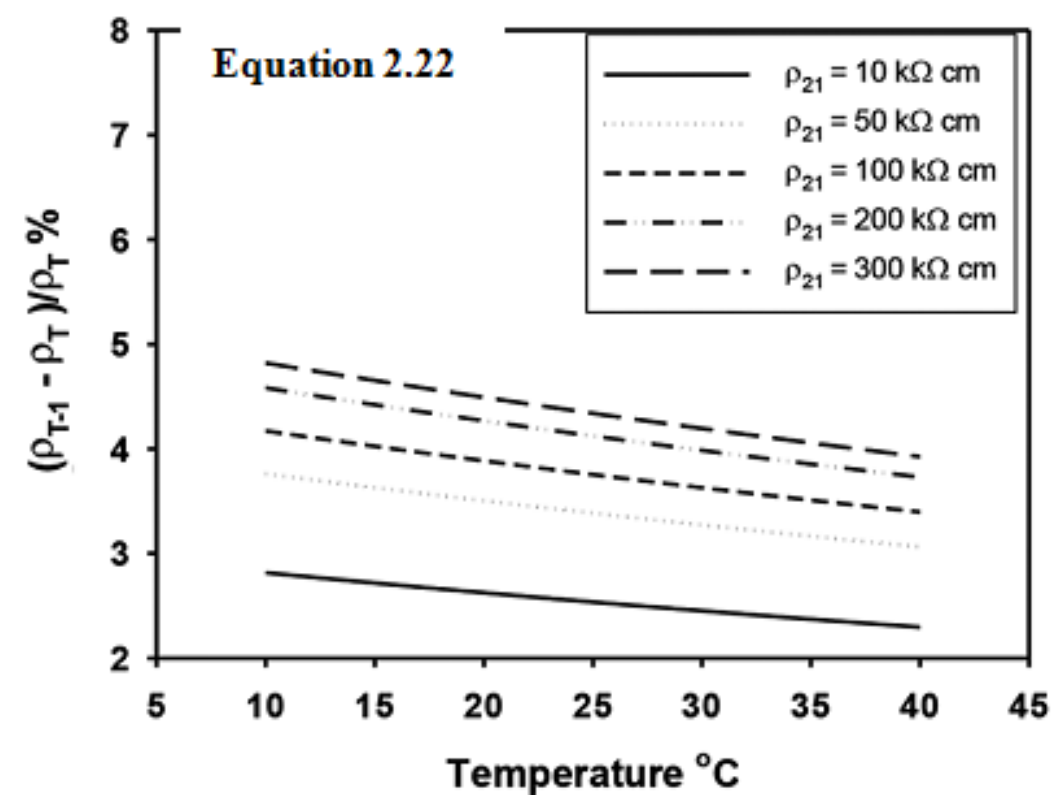

(a) 


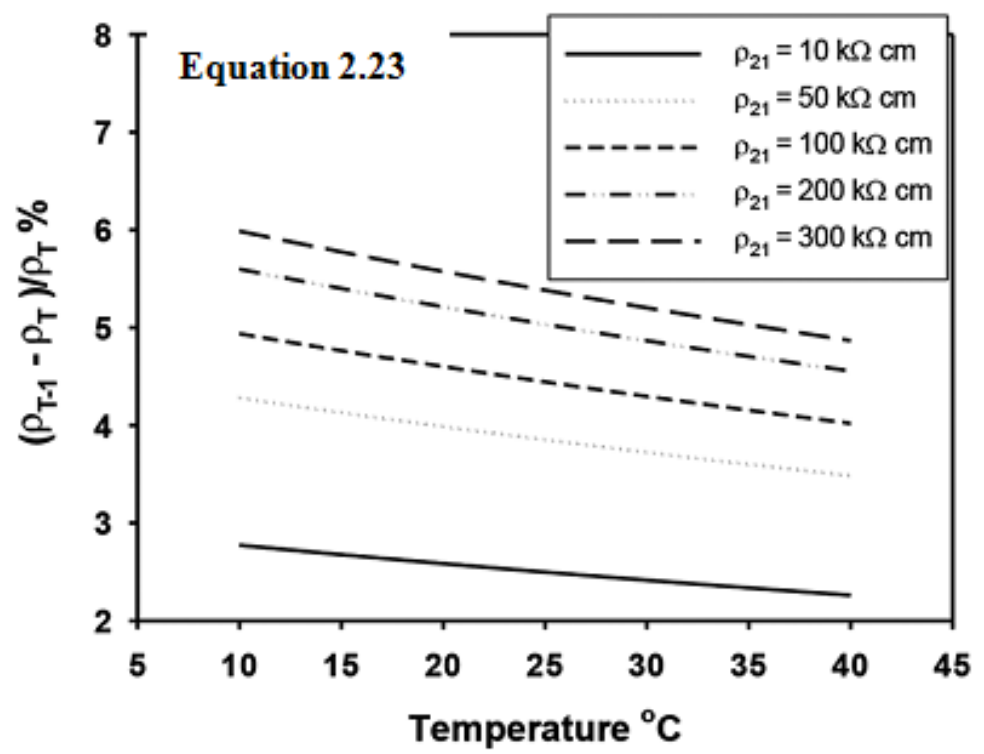

(b)

Figure 2.19. Calculated percentage change in resistivity per ${ }^{\circ} \mathrm{C}$ using: a) Eq. 2.22; b) Eq. 2.23 [7].

\subsection{Effect of supplementary cementitious materials and chemical admixtures on electrical resistivity}

The practice of using SCMs such as silica fume, fly ash and slag has been growing for the past three decades. They are mostly byproducts of other industrial processes. Their judicious use is desirable not only from the sustainable development point of view, but also for the technical benefits they provide to concrete. They are used to improve various characteristics of concrete in fresh or hardened state. Following, studies conducted on the effect of SCMs on the electrical resistivity of concrete in fresh state are presented.

Li et al. [15] observed that fly ash containing concretes have higher electrical resistivity than that of concrete without fly ash until final setting time during which resistivity is governed by pore solution conductivity not the change of porosity (see Fig. 2.14). 
Because fly ash retards and lessens the chemical reaction and hydration; thus, less ionic species are present in pore solution which leads to increase in resistivity. However, after final setting time and during hardening another mechanism occurs. At this stage and afterward because the governing factor in resistivity would be microstructure change due to the formation of hydration products, fly ash containing concrete shows smaller resistivity which indicates greater porosity as a result of less production of hydration products (solid phase). They also observed that higher replacement of fly ash in concrete resulted in lower associated resistivity. Additionally, the hydration degree, as shown in Fig. 2.15, was reported to be reduced [15] by adding fly ash as a replacement of cementitious materials.

Mancio et al. [8] conducted the electrical resistivity measurement test on concrete samples with various w/c ratios of $0.3,0.4,0.5$ and 0.6 with $0 \%$ and $25 \%$ of fly ash. They monitored electrical resistive of fresh concrete during the first 2 hours using the Wenner probe. As shown in Fig. 2.8, they observed that for a fixed w/c ratio and aggregate volume fraction, electrical resistivity of fly ash incorporated fresh concrete (before setting and hardening time) were always higher than that of mixtures without fly ash. They reported that this higher resistivity is attributed to slow rate of pozzolanic reactions of fly ash which leads to a less ionic concentration in the pore solution. As a result, pore solution electrical resistivity increases which is a dominant parameter in determining the bulk resistivity of fresh concrete before hydration products formation.

The silica fume addition leads to the formation of the $\mathrm{CSH}$ phases which are the result of active silica fume reaction with free $\mathrm{Ca}(\mathrm{OH})_{2}$ released as a cement hydration product 
[52,53]. Salem et al. [10] studied electrical conductivity of the cement pastes made of slag and Cement Kiln Dust (SL/CKD ratio of 80/20) with and without silica fume. Conductance of specimens was measured during first 24 hours of hydration age. They observed that the conductance increased at very early age to a peek value and then decreased. It was reported that silica fume replacement from $2 \%$ to $20 \%$ resulted in increasing conductivity of paste, which was attributed to the higher hydraulic reactivity of silica fume compared to slag. In general higher reactivity of the cementitious materials results in more concentration of transporting ions in the pore solution.

Furthermore, Salem in another study [9] observed that increasing silica fume from 10 to 50 percent replacement in OPC pastes decreased the conductivity at the early stage (see Fig. 2.16). This conductivity decrease was attributed to the delayed reactions of silica fume compared to $\mathrm{OPC}$ in hydration process.

Bekir et al. [13] studied the electrical conductivity of cement pastes at early age (1 day). They investigated the effect of supplementary cementitious materials added to OPC such as fly ash, silica fume, and blast furnace slag (BFS). During the initial stage of hydration time (before hardening) the governing parameter for conductivity is the hydrolysis (dissolution) of the OPC components which results in releasing ions such as $\mathrm{Ca}^{2+}, \mathrm{OH}^{-}$, $\mathrm{SO}_{4}{ }^{2-}$, and alkali ions (i.e. $\mathrm{K}^{+}$and $\mathrm{Na}^{+}$) [9]. Bekir et al. [13] reported that at a certain w/c ratio, conductivity of the paste at early age decreases if it contains SCMs such as fly ash, silica fume and slag. They suggested that slower pozzolanic reaction with water and hence release of less ion as well as decreasing volumetric fraction of the liquid phase because of increased volume of solid phase due to less specific gravity of SCMs 
compared to OPC, contributed to this decrease in conductivity. Also, it was reported that the rate of change in electrical conductivity decreased as the replacement ratio of SCM in total cementitious materials increased.

Bekir at al. [13] also found that among the SCMs, the reduction in conductivity at early stage is more significant in fly ash, slag and silica fume, respectively. However, in advanced phase of hydration (i.e., hardening) fly ash and silica fume added pastes had lower electrical conductivity (more resistivity) than slag-blended admixtures because of their faster pozzolanic reactions compared to the pastes with slag [10].

The application of retarders in concrete, as a chemical admixture, is to delay the setting time of mixture so that the workable period for construction team increases. Using a noncontact electrical resistivity measurement [4], Li et al. [26] monitored the resistivity development of cement pastes incorporated with retarder of different dosages of $0 \%$, $0.1 \%, 0.15 \%$ and $0.2 \%$ (by weight with respect to the solid content) at fixed $\mathrm{w} / \mathrm{c}$ ratio of 0.3 during first 24 hours. They reported that the minimum point $\left(\mathrm{P}_{\mathrm{m}}\right)$ resistivity was not significantly affected by adding retarder from which it was concluded that the total number of ions soluble in water at the end of dissolution period was not considerably influenced by retarder incorporation. Whereas, after setting and hardening, the resistivity of pastes with higher dosage of retarder was always lower than that of lower content of retarder (see Fig. 2.5). Because the hydration products were formed slower in pastes containing retarder which led to porous media with less tortuosity and thus corresponding resistivity decreased. 
For practical purposes, one of the key points in designing a concrete mixture is to select the most suitable superplasticizer in terms of type and optimum dosage. The workable period of concrete is desired to be more than mixing, transportation, cast into formworks and finishing time so that there will not be any practical difficulties as a result of setting and hardening of concrete. Therefore, the effect of superplasticizers can significantly favor this objective. Torrents et al. [23] qualitatively studied the effect of superplasticizer addition in OPC pastes. They monitored electrical resistivity of pastes with w/c of 0.33 containing superplasticizer dosages of $0 \%, 0.5 \%, 1 \%$, and $4 \%$ during first 24 hours of hydration age. It was reported that superplasticizer incorporation delays final setting time of OPC pastes and so the accelerated electrical resistivity gain. This was attributed to slower hydration rate of OPC in presence of superplasticizer which resulted in delayed formation of hydration products.

Xiao et al. [25] adopted a similar method to a previous study [52] and developed it to select a suitable superplasticizer based on the results derived from electrical resistivity measurements. For the fixed w/c ratio of 0.3 , using a non-contact electrical resistivity measurement technique, they monitored electrical resistivity development of pastes incorporated with different dosages of two types of superplasticizer SP1 (Naphthalenebased) and SP2 (Polycarboxilate) during the first 24 hours as shown in Fig. 2.20. The saturation dosage (weighted ratio to cement mass) of each superplasticizer was chosen by Marsh cone test which suggested the maximum dosage to be used in cement paste or concrete (e.g. $0.8 \%$ for SP1 and $0.25 \%$ for SP2). 

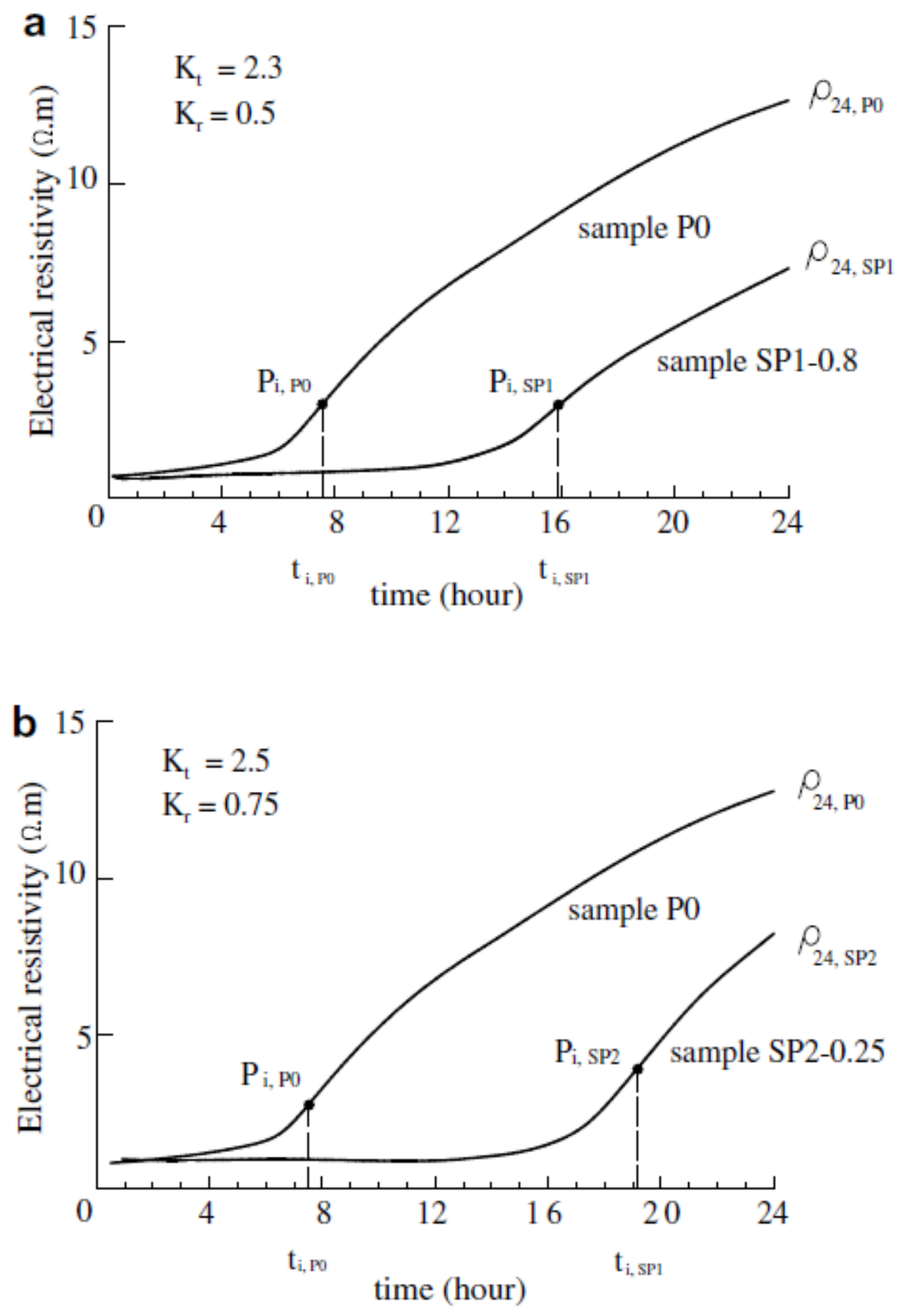

Figure 2.20. Electrical resistivity development and inflection point $\left(\mathrm{t}_{\mathrm{i}}\right)$ identification for control paste ( $\left.\mathrm{P}_{0}\right)$ with: a) $0.8 \%$ of SP1; and b) $0.25 \%$ of SP2 [25]. 
It was reported by Xiao et al. [25] that adding superplasticizer prolongs the setting time and decreases electrical resistivity of the cement paste. Accordingly, the best superplasticizer was claimed to be the one that could delay the setting time more to provide more workable period, as well as having rapid strength gain after hardening which corresponds to greater electrical resistivity at 24 hours of hydration age. Based on these criteria, they proposed inflection time ratio $\mathrm{K}_{\mathrm{t}}$ and resistivity ratio $\mathrm{K}_{\mathrm{r}}$ as follows:

$$
\begin{gathered}
K_{t}=\frac{t_{i, S P}}{t_{i, P 0}} \\
K_{r}=\frac{\rho_{24, S P}}{\rho_{24, P 0}}
\end{gathered}
$$

where $t_{i, S P}$ and $t_{i, P O}$ are inflection times of control paste without superplasticizer and paste incorporated with superplasticizer, respectively; $\rho_{24, \mathrm{SP}}$ and $\rho_{24, \mathrm{P} 0}$ are resistivity of control paste and paste containing superplasticizer, respectively. Xiao et al. [25] suggested that the superplasticizer which results in higher inflection time ratio $\mathrm{K}_{\mathrm{t}}$ and resistivity ratio $\mathrm{K}_{\mathrm{r}}$ would be the better and more cement compatible choice.

Furthermore, Li et al. [6] tested 11 specimens with different mixture proportions. The results of resistivity measurements and corresponding time of critical points $\mathrm{P}_{\mathrm{m}}$ (minimum point on resistivity-time curves) and $\mathrm{P}_{\mathrm{t}}$ (maximum curvature point on log-scale resistivity-time curves) as well as conventional setting times obtained by penetration method are presented in Table 2.1. They concluded that superplasticizer in concrete performed as a retarder that deferred the initial and final setting times. It was also 
observed that fly ash increased the initial and final setting times of concrete because of delay in the formation of hydration products due to delayed pozzolanic reactions.

$\mathrm{Li}$ et al. [6] also observed that in the specimens containing $\mathrm{CaCl}_{2}$ as an accelerator, the initial and final setting times occurred earlier compared to concrete specimen without accelerator. It contributed to faster hydration products formation resulting from more $\mathrm{Ca}^{2+}$ presence in the paste.

In addition, $\mathrm{Li}$ et al. [5] reported that $1 \% \mathrm{KCl}$ by weight of cementitious materials, as an accelerator changed the fresh and hardened resistivity of paste with the same w/c ratio of 0.4. Figure 2.3 illustrates that increasing ions concentration in liquid phase because of $\mathrm{K}^{+}$ and $\mathrm{Cl}^{-}$release at the fresh state resulted in a rise in conductivity and a drop in resistivity. However, after setting initiation time when porosity decreased because of solidification, the resistivity of $\mathrm{KCl}$ containing paste became higher than that of $\mathrm{KCl}$-free paste with the same $\mathrm{w} / \mathrm{c}$ ratio. This demonstrated the dominant role of microstructure change instead of ions concentration during the setting and hardening stages.

\subsection{Relationship between bulk electrical resistivity and pore solution resistivity: Archie's Law and Formation Factor F}

The electrical resistivity of typical aggregates used in concrete ranges from $10^{3}$ to $10^{12}$ $\Omega . \mathrm{m}$ [1], while that of a moist cement paste ranges from 10 to $13 \Omega . \mathrm{m}$ resulting in a range of 25-45 $\Omega . \mathrm{m}$ for concrete electrical resistivity [16]. Since the resistivity of aggregates are several orders higher than paste resistivity, almost all current passes through cement paste with a much lower resistivity. Therefore, the resistivity of composite $\left(\rho_{m}\right)$ such as 
concrete, can be related only to its dominant component, paste matrix $\left(\rho_{\mathrm{p}}\right)$, and its fractional volume $(\varphi)$ as follows [16]:

$\rho_{m}=\frac{\rho_{p}}{\varphi \lambda}$

where $\lambda$ is a reduction factor which is less than 1 and is derived from experiment.

In a composite material with a cement paste matrix and aggregates with a relative conductivity close to zero compared to the cement paste, the formation factor (F) is defined (Eq. 2.27) as the ratio of the composite resistivity $\rho_{x}$ to the cement paste resistivity $\rho_{1}$.

$F=\frac{\rho_{x}}{\rho_{1}}$

Many researchers tried to establish a formula for formation factor $F$ as a function of $\varphi$, fractional volume of cement paste matrix or in general, fractional volume of the conductive component. They are based on the assumptions made on the shape of the particles and their distribution in matrix.

Maxwell [53] proposed the formation factor $\mathrm{F}$ for spherical particles in matrix with large distant compared to their radius as

$F=\frac{3-\varphi}{2 \varphi}$

Slawinski [54] derived an empirical relationship for spheres both in contact and dispersed as follows: 
$F=\frac{(1.3219-0.3219 \varphi)^{2}}{\varphi}$

Fricke [55], studying particular sand as nonconductive particles, developed following F- $\varphi$ relationship to be applicable for spheres, as well as oblate and prolate spheroids:

$F=\frac{(x+1)-\varphi}{x \varphi}$

where $\mathrm{x}$ can be obtained from experiments for a particular type of particle and is equal to 2 for spherical particles. $\mathrm{x}$ is less than 2 for spheroids (e.g. the sand tested by Fricke [55] revealed $\mathrm{x}$ as 1.4).

Although the concrete can be modeled as particles distributed in cement paste matrix, the size and shape of aggregates vary (not single size and shape particle) and therefore, more advanced approach is required.

Archie [19], working on resistivity of consolidated and unconsolidated sandstones proposed Eq. 2.31 for sandstones $100 \%$ saturated with water as follows:

$F=\frac{\rho_{0}}{\rho_{w}}=\varphi^{-m}$

where $\rho_{0}$ is the resistivity of sandstone $100 \%$ saturated with water; $\rho_{\mathrm{w}}$ is the resistivity of water contained in the sandstone; $\varphi$ is the fractional volume of water (conductive component) contained in the rock; and $\mathrm{m}$ is the shape factor.

Pirson [56] suggested that exponent m ranges from 1.3 to 2.2 for slightly and highly cemented rocks. Atkins and Smith [57] generalized Archie's equation (Eq. 2.31) for 
multi-size particles adding constant A which is indicative of the shape and distribution of aggregates:

$F=A \varphi^{-m}$

Whittington et al. [16], applying Archie's equation for continuously moist cure concretes of different mix proportions and $\mathrm{w} / \mathrm{c}$ 's, reported the following relationship:

$F=1.04 \varphi^{-1.20}$

where 1.04 and 1.20 are respectively the values of $\mathrm{A}$ and $\mathrm{m}$ obtained in their study.

Li et al. [5] measured resistivity of the extracted pore solution $\rho_{0}(t)$ of pastes with $0.3,0.4$ and $0.5 \mathrm{w} / \mathrm{c}$ ratios at times of corresponding minimum critical points 21,26 and $33 \mathrm{~min}$, respectively. Using the Archie's law [19], they established relationship between formation factor $\mathrm{F}$ and porosity $\varphi$ of pastes as follows:

$F=0.68 \varphi^{-1.8}$

Li and Wei [14], from the electrical analogy point of view, considered the cement paste as a tow-component parallel materials; i.e., cementitious materials or solid content with a very high resistivity from $10^{3}$ to $10^{12} \Omega . \mathrm{m}$, as reported by Whittington et al. [16], and liquid content whose resistivity ranges from 0.25 to $0.35 \Omega$.m, as reported by Buenfeld and Newman [58]. Hence, if the liquid content, pore solution, is regarded as a conductive component compared to solid phase with conductivity close to zero, cementitious materials can be assumed as nonconductive component with volumetric fraction of $\mathrm{V}_{\mathrm{nc}}$. 
For the $100 \%$ degree of saturation in which the entire volume of pores is filled with water, porosity $(\varphi)$ equals the volumetric ratio of liquid or conductive component $\left(\mathrm{V}_{\mathrm{c}}\right)$. Therefore, using the parallel resistants law $\left(\mathrm{R}_{\mathrm{c}}\right.$ and $\left.\mathrm{R}_{\mathrm{nc}}\right)$ to obtain the equivalent resistivity, cement paste bulk resistivity ( $\rho$ ) can be expressed as follows [14]:

$\frac{1}{R}=\frac{a}{R_{c}}+\frac{b}{R_{n c}}$

$\frac{1}{\rho}=\frac{C_{1} V_{c}}{\rho_{c}}+\frac{C_{2} V_{n c}}{\rho_{n c}} \approx \frac{C_{1} V_{c}}{\rho_{c}}=\frac{V_{c}^{m}}{\rho_{c}}=\frac{\phi^{m}}{\rho_{c}}$

where $R_{c}, R_{n c}$, and $R$ are the resistances of pore solution, cementitious materials, and cement paste in $\Omega$, respectively; $\rho_{\mathrm{c}}$ and $\rho_{\mathrm{nc}}$ are the resistivities of pore solution and cementitious materials in $\Omega . m$, respectively, and a, b, $\mathrm{C}_{1}$ and $\mathrm{C}_{2}$ are the constants representing the geometry of the equivalent parallel model.

Eq. (2.36) shows Archie's Law [19] which was proposed in 1942 to relate the resistivity of sand stones saturated with conductive water to its porosity. Using electrical analogy above, one can see the application of Archie's Law for cement paste. A very significant point to note is that because of the hydration process, all terms in Archie's Law for the cement paste are the function of time ( $\mathrm{t}$ ) and therefore, they need to be determined at specific time; e.g. 30 minutes after mixing. Thus, to take into account the effect of time in cement paste, Eq. 2.37 was proposed [14]. It illustrates that the bulk resistivity of the cement paste increases as the pore solution resistivity increases or porosity decreases.

$$
\rho(t)=\rho_{o}(t) \varphi(t)^{-m(t)}
$$




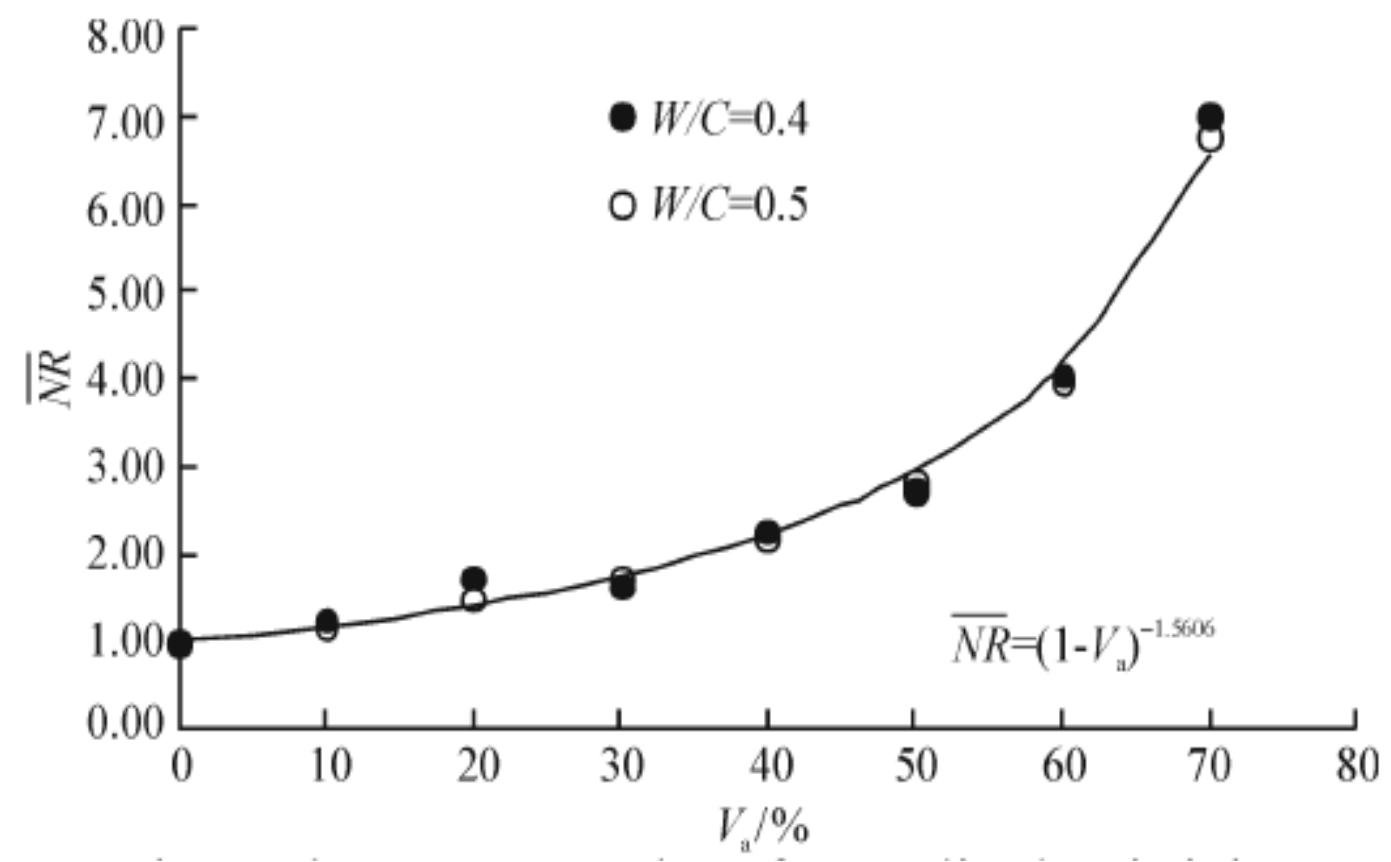

Figure 2.21. The average of normalized resistivity $(\overline{N R})$ as a function of aggregate volume fraction $\left(\mathrm{V}_{\mathrm{a}}\right)$ for concrete samples [27].

Wei and Xiao [27] observed the same trend of cement paste for the electrical resistivity development with time in concrete with various aggregate volume fractions, $V_{a}$; i.e., the curves dropped to a minimum point and then gradually increased with time which was governed by hydration process of its paste matrix. Therefore, they decided to study the relationship between electrical resistivity of concrete $\rho_{\mathrm{C}, \mathrm{t}}$ and paste matrix $\rho_{\mathrm{P}, \mathrm{t}}$ as a two component system; (1) aggregates $\left(\mathrm{V}_{\mathrm{a}}\right)$ and (2) paste matrix $\left(1-\mathrm{V}_{\mathrm{a}}\right)$. They expressed the normalized resistivity (NR) of concrete to its paste matrix as follows:

$$
N R=\frac{\rho_{C, t}}{\rho_{P, t}}
$$


This ratio can be considered as the formation factor defined in Archie's law. Wei and Xiao [2] suggested that the average of normalized resistivity $\overline{N R}$ derived from reproducibility tests is time-independent (constant during first 24 hours) and w/c ratio also does not affect it. It only depends on the aggregate volume fraction $\mathrm{V}_{\mathrm{a}}$ and increases by increasing fraction of aggregates as shown in Fig. 2.21. Therefore, by measuring the electrical resistivity of concrete,$\rho_{\mathrm{C}}$, and knowing the volumetric fraction of aggregates, $\mathrm{V}_{\mathrm{a}}$, electrical resistivity of paste, $\rho_{\mathrm{P}}$, can be determined via:

$\rho_{C}=\rho_{P}\left(1-V_{a}\right)^{-1.5606}$

\subsection{Aggregate volume effect on the electrical resistivity}

The resistivity of typical aggregates in concrete are several orders higher than the paste resistivity [16]. Whittington et al. [16] concluded that the resistivity of concrete is almost entirely dependent on the resistivity of cement paste, because the resistivity of aggregates is infinite compared to that of the cement paste. Hence, the resistivity of the concrete as a composite material is about 3 to 4 times of resistivity of its cement paste. Therefore, the paste fraction in mixture design affects the resultant resistivity of concrete; i.e., increase in percentage of paste (cement plus water) in concrete mixture increases the conductivity and decreases the corresponding resistivity [16].

Although Mancio et al. [8] did not study the effect of aggregate volumetric fraction in concrete, the mixture proportion of their samples presented in Table 2.3 reveals that in the experiments, the aggregate volume fraction $\left(\mathrm{V}_{\mathrm{a}}\right)$ also increased along with w/c ratio. 
Therefore, higher volumetric fraction of aggregates most-likely was the governing effect on the electrical resistivity rise seen in Fig 2.8 , not increase in w/c ratio as reported.

Wei and Xiao [27] investigated the effect of aggregate volume on the electrical resistivity of fresh concrete during first 24 hours. Using a non-contact electrical resistivity measurement [4], they monitored electrical resistivity of sixteen different mixtures with various aggregate volume fraction from $0 \%$ to $70 \%$ at w/c ratios of 0.4 and 0.5 . In Fig. 2.22, the two digit number in samples labels indicates the w/c ratio followed by the volumetric fraction of aggregates; e.g. C41 and C50 represent concrete samples with w/c of $0.4,10 \%$ aggregate volumetric fraction and $\mathrm{w} / \mathrm{c}$ of $0.5,0 \%$ aggregate volumetric fraction, respectively. The types of aggregates were same for all the mixtures (i.e., the coarse and fine aggregates were made of granite and quartz, respectively). Wei and Xiao [27] observed that for each $w / c$ ratio, increasing the aggregate volume fraction $V_{a}$ significantly increased the electrical resistivity; i.e., the higher the aggregate volume fraction, the higher the resistivity of concrete sample (see Fig. 2.22). They also found that the resistivity development trend for the concrete was similar to that for the paste. It dropped to a minimum value and gradually started to rise as cement hydrated which confirmed the dominant role of cement paste matrix in determining the electrical resistivity of concrete. 


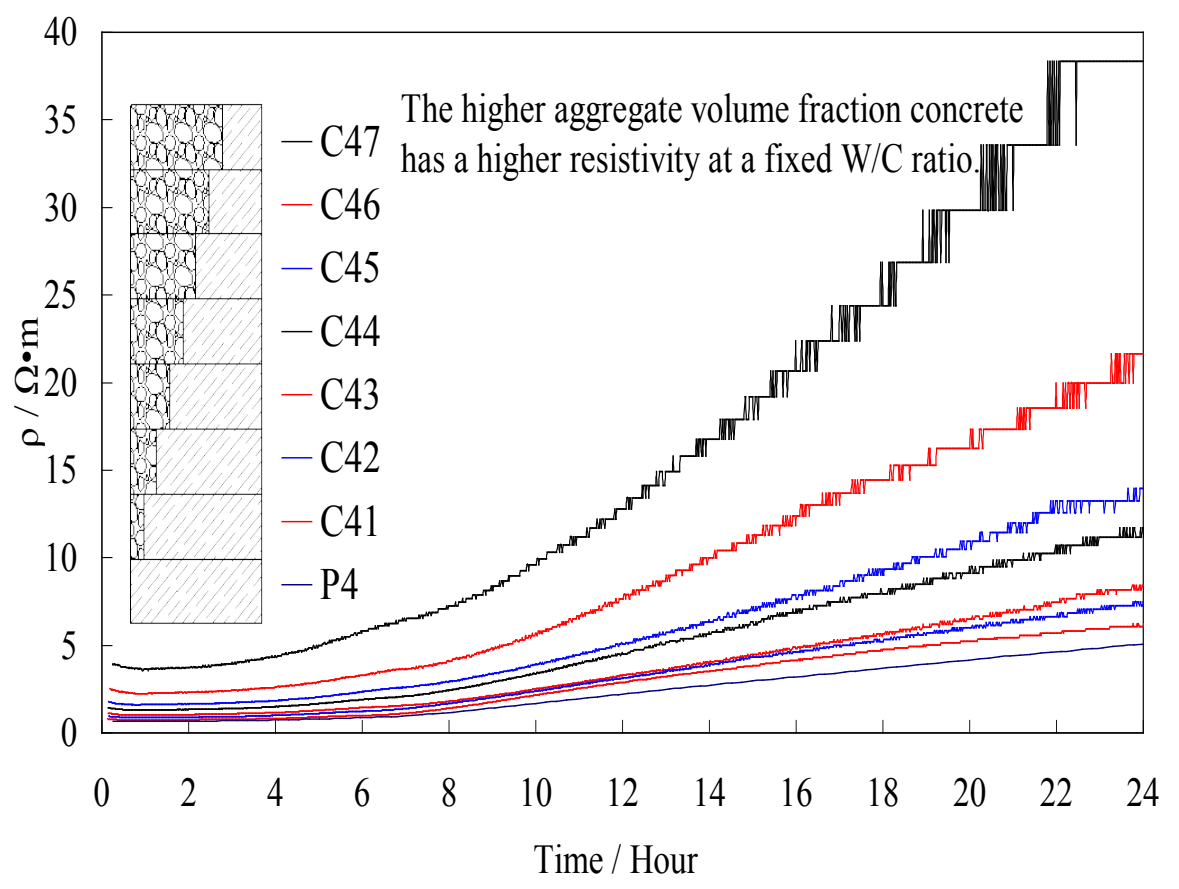

(a)

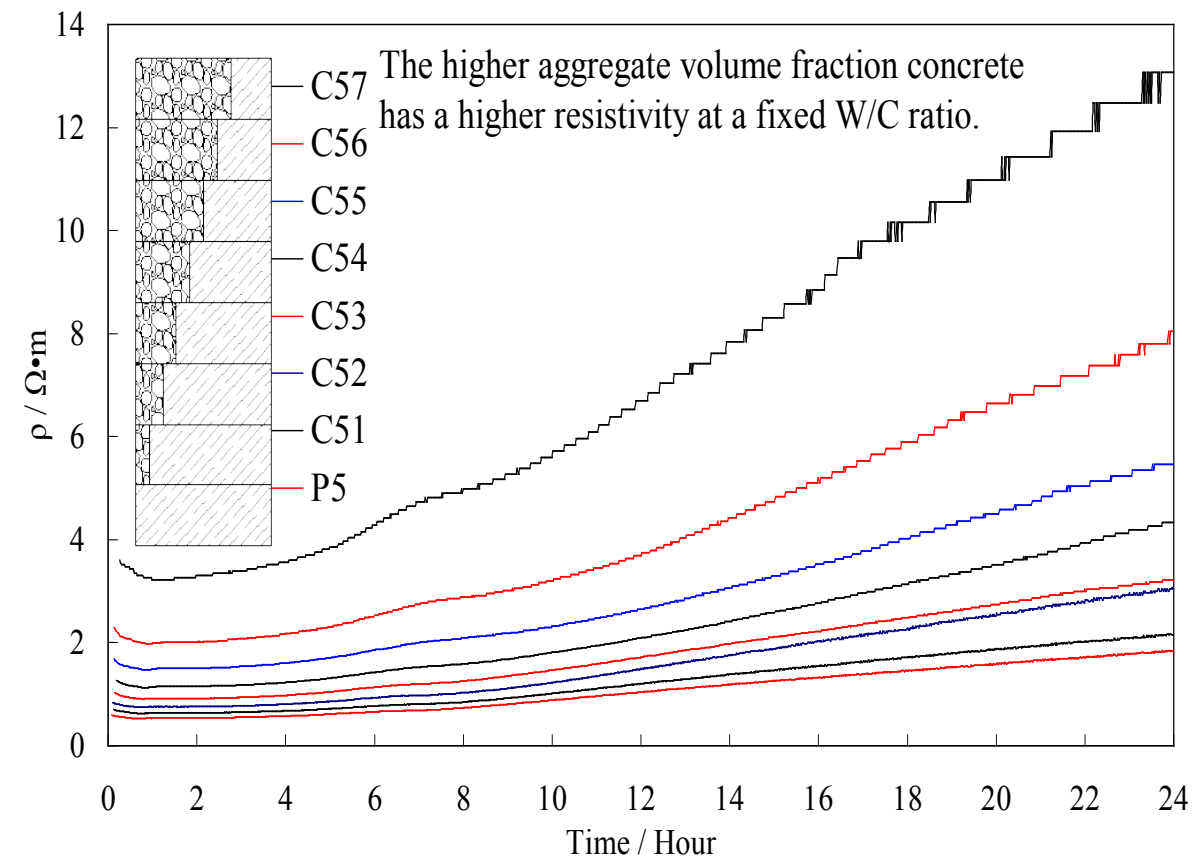

(b)

Figure 2.22. Electrical resistivity development with time during 24 hours for concrete samples with different aggregate volume fraction $\mathrm{V}_{\mathrm{a}}: \mathrm{a}$ ) $\left.\mathrm{w} / \mathrm{c}=0.4, \mathrm{~b}\right) \mathrm{w} / \mathrm{c}=0.5$ [27]. 


\subsection{Summary and gaps in literature}

The electrical resistivity has been used to evaluate behavior of cement based materials such as cement paste, mortar or concrete as early as 1950 s. The governing component and conductive portion in concrete has been observed to be cement paste with very low resistivity compared to aggregates. Therefore, to evaluate the characteristics of concrete using electrical resistivity method, the best approach is to focus on the conductive part of concrete; i.e., cement paste. Based on this literature review, some gaps in the area of concrete electrical resistivity have been identified for further studies in this research as follows:

2.10.1. w/c ratio effect on electrical resistivity and applicability of Archie's law in fresh cement paste

The bulk electrical resistivity of paste which has two components including liquid phase (pore solution) and solid phase (cementitious materials) is the function of pore solution resistivity (conductive component), volumetric fraction of pore solution, porosity, and tortuosity which can be obtained from experiments. This relationship (Eq. 2.31) was proposed and confirmed by Archie [19] for the porous rocks and some researchers later investigated applicability of that for hardened concrete. Electrical resistivity of pore solution and paste are time dependent because of chemical reactions. Accordingly, any conclusion about the effect of a specific parameter such as porosity should be drawn at a specific hydration age to eliminate the effect of hydration progress, release of ions and solidification. Li et al. [5] observed that resistivity of pore solutions extracted from pastes with w/c of $0.3,0.4$ and 0.5 at their times of minimum critical points $(21,26$ and 33 
minutes, respectively), increase by increasing w/c ratio. However, since the time itself affects the pore solution resistivity due to further dissolution of ions into pore solution, measurements should have been taken at a specific time to have more reliable data.

Furthermore, they concluded [26] that with increase of retarder dosage, the ion concentration in pore solution increased and corresponding resistivity decreased (see Table 2.5). This conclusion contradicted with the fact that slower hydration reactions in greater dosages of retarder would result in less concentration of ions in pore solution and accordingly less conductivity which corresponds to more resistivity. This decrease in resistivity was most likely because of longer time of pore solutions extraction in pastes with higher portion of retarders (i.e., 16, 51, 81, and 178 minutes for $0.00 \%, 0.10 \%$, $0.15 \%$, and $0.20 \%$ of retarder dosage, respectively) and thus more ions released as a result of hydration progress, which increased the conductivity, not greater dosage of retarders. Thus, the applicability of Archie's law in fresh cement paste, before setting time, has not been completely studied.

The effect of w/c ratio on electrical resistivity of paste and concrete after setting and hardening was studied by several researchers $[14,17]$. However, very few of them such as Li et al. [5] studied the electrical resistivity at a very early age before setting time. Mancio et al. [8] came up with an contradictory conclusion that increasing w/c ratio in concrete samples increases electrical resistivity of concrete. However, the mixture proportion of these samples (Table 2.3) shows that in the experiments, the aggregate volume fraction also increased along with w/c ratio which most-likely is the governing effect on the electrical resistivity rise (as discussed in Section 2.9) not increase in w/c 
ratio. Furthermore, the best approach to see the effect of w/c ratio would be using formation factor which reflects the ratio of bulk electrical resistivity to pore solution resistivity.

\subsubsection{Effect of SCMs on electrical resistivity in fresh state}

Nowadays, the SCMs and chemical admixtures are inevitable portions of concrete products because of various technical and practical advantages. Their effect on the electrical resistivity of concrete has been investigated by several researchers $[9,10,13$, 25]. However, the results from some researchers do not support the others' and some contradictions were observed in some cases which show the need for further study and work on this topic. On the other hand, the main focus of these studies was not on fresh state; they were more interested in hardening state (i.e., after setting time). For instance, Salem et al. [10] reported that by increasing w/c ratio of cement kiln dust-silica (CKD) fume incorporated pastes which represents their porosity, conductivity increased which correlates to decrease in resistivity. They concluded that this increase in conductivity was attributed to the increase of hydrolysis degree of CKD constituents leading to an increase in the concentration of ions in pore solution. However, this conclusion could be misleading because the concentration of ions per unit volume of paste decreased by increasing $w / c$ ratio and the governing effect most likely is the greater portion of conductive phase (pore solution) compared to solid phase (i.e., physical effect) which eventually increased conductivity of the paste. In addition, Bekir et al. [13] also found that among the SCMs, the reduction in conductivity at early stage is more significant in FA compared to BFS and SF. This observation is not consistent with those reported by 
other people. Therefore, extensive research on the effect of SCMs is still needed to clarify some of the existing inconsistency in the literature.

\subsubsection{Simple model for the estimation of pore solution conductivity}

The cement paste can be regarded as a two-component system: conductive pore solution; and non-conductive cementitous materials $[14,16]$. Thus, the charge transport occurs through the pore solution by the ions released as a result of chemical reaction between cementitous materials and water. Hence, the pore solution conductivity determines the

corresponding resistivity of cement paste. However, the experimental measurement of pore solution conductivity is tiresome and time consuming because of laborious process of solution extraction from paste. Therefore, there is a considerable demand in construction industry for a simple model which can predict pore solution conductivity with time. But, there is no model in the literature that can accurately estimate pore solution conductivity during fresh state. Current study also proposed this model as well as its experimental validation for various paste mixtures including OPC incorporated with commonly used SCMs and superplasticizer.

\subsubsection{Conductivity of pore solution versus time: Fresh and hardened state}

The laborious and time consuming procedure of pore solution extraction from cement paste, has probably been the reason that conductivity of pore solution has not been studied at various times of hydration age; this scenario is even more difficult for lower w/c ratio pastes specially in hardened state. If the variation of conductivity of pore solution with time could be monitored, it would provide researchers with very valuable 
information about the progress of chemical reactions during hydration in cement paste. Therefore, current study also investigated the change of pore solution conductivity with time during fresh state as well as its comparison to hardened state conductivity.

\subsection{5.pH measurement as an alternative to obtain the conductivity of pore}

\section{solution at fresh state}

The results from literature [12] suggested that the concentration of $\mathrm{OH}^{-}$in the pore solution as the most contributing ion to the conductivity, is dominant in total pore solution conductivity. The measurement of conductivity of pore solution is a tedious job because of the extraction process; however, $\mathrm{pH}$ measurement in fresh concrete or cement paste is fast and very easy and it provides the $\mathrm{pH}$ of the pore solution; i.e., the $\mathrm{pH}$ of concrete, cement paste and pore solution are identical. Therefore, development of a relationship between $\mathrm{pH}$ and conductivity of pore solution enables us to indirectly calculate pore solution conductivity from the $\mathrm{pH}$ of fresh cement paste or concrete, and the difficulties associated with the extraction of pore solution (i.e., tiresome and time consuming) are eliminated. 


\section{Experimental Plan}

\subsection{Introduction}

In the current study, the main focus was the electrical behaviour of cement paste because it is the conductive component of concrete and its electrical characteristics play an important role on the electrical properties of fresh concrete. Therefore, all the measurements and monitoring were conducted on fresh paste during the first two hours after mixing before the initial setting time that occurs as a result of cement hydration. During this period the electrical resistance of the fresh concrete is relatively stable. Since the total conductivity/resistivity of the pore solution phase of the cement paste is related to the concentration and mobility of ions, in particular the hydroxide ion $\left(\mathrm{OH}^{-}\right)$[12], the $\mathrm{pH}$ of both cement paste and its pore solution were also measured at specific times to establish a relationship between $\mathrm{pH}$ and conductivity of pore solution.

\subsection{Materials}

Cement paste has solid (cementitious materials) and liquid (water) components. The water used in construction is usually tap water; however, in our tests the distilled water was used to have consistency among all mixtures and eliminate the effect of soluble ions in tap water. The conductivity of distilled water was $42.5 \times 10^{-3} \mathrm{mS} / \mathrm{cm}$, which was quite low indicating that it did not contain any significant source of ions. It had several order of magnitude smaller conductivity than the paste and pore solution.

Superplasticizers are frequently used in the concrete industry to increase the workability and reduce the water content of concrete mixtures. Therefore, it was important that the 
effect of superplasticizer was also included in this research. One of the most common commercial superplasticizer manufactured by BASF (a chemical admixture company), called MasterGlenium 3030, was used in the cement paste mixtures tested in this experimental program. The density of "MasterGlenium 3030" was reported by the manufacturer to be close to that of water (i.e., about $1 \mathrm{~g} / \mathrm{cm}^{3}$ ) and its conductivity was $5.42 \mathrm{mS} / \mathrm{cm}$. Since the superplasticizer was only about $1 \%$ (by mass) of the liquid phase, the corresponding conductivity in water was recorded as $102.7 \times 10^{-3} \mathrm{mS} / \mathrm{cm}$, which was rather low, indicating that ionic concentration in the liquid phase before mixing with cementitious materials was not significant.

The solid part of the paste (i.e., the cementitious materials), includes cement Type I as ordinary portland cement (OPC) according to the ASTM standard and supplementary cementitious materials (SCM). The chemical and physical properties of these materials are shown in Table 3.1. Three most commonly used SCMs were selected in our tests to take into account their effect on the electrical behavior of cement paste system: namely fly Ash, silica fume and slag in this study.

\subsection{Sample Preparation}

\subsubsection{Cement paste mixtures}

Five types of cement paste mixtures were prepared in this study: (1) OPC, (2) OPC plus superplasticizer, (3) OPC plus fly ash, (4) OPC plus silica fume, and (5) OPC plus slag. Altogether, 54 different paste samples were prepared and tested. In addition, 17 repetitive pastes were made to test the reliability of the data obtained from experiments. In Table 
3.2, the detailed proportions of all 54 cement paste mixtures used in this study are presented. Labeling convention and the background on the selection of these mixtures are described below.

Table 3.1. Chemical and physical properties of cementitious materials.

\begin{tabular}{|c|c|c|c|c|}
\hline & $\begin{array}{c}\text { OPC } \\
(\% \text { of mass })\end{array}$ & $\begin{array}{l}\text { Silica Fume } \\
\text { (\% of mass) }\end{array}$ & $\begin{array}{c}\text { Fly Ash } \\
(\% \text { of mass })\end{array}$ & $\begin{array}{c}\text { Slag } \\
(\% \text { of mass })\end{array}$ \\
\hline $\mathrm{Na}_{2} \mathrm{O}$ & 0.08 & 0.08 & 0.94 & 0.30 \\
\hline MgO & 3.23 & 0.47 & 1.28 & 10.09 \\
\hline $\mathrm{Al}_{2} \mathrm{O}_{3}$ & 4.74 & 0.19 & 18.37 & 7.95 \\
\hline $\mathrm{SiO}_{2}$ & 19.74 & 93.50 & 36.56 & 37.84 \\
\hline $\mathbf{P}_{2} \mathbf{O}_{5}$ & 0.06 & 0.11 & 0.13 & 0.01 \\
\hline $\mathrm{SO}_{3}$ & 3.06 & 0.04 & 0.63 & 0.48 \\
\hline $\mathbf{K}_{2} \mathbf{O}$ & 0.56 & 0.92 & 1.78 & 0.42 \\
\hline $\mathrm{CaO}$ & 63.68 & 0.30 & 3.56 & 39.09 \\
\hline $\mathrm{TiO}_{2}$ & 0.26 & 0.02 & 0.80 & 0.79 \\
\hline MnO & 0.04 & 0.11 & 0.23 & 0.47 \\
\hline $\mathrm{Fe}_{2} \mathrm{O}_{3}$ & 1.8 & 1.56 & 32.47 & 0.47 \\
\hline $\begin{array}{l}\text { Particle density } \\
\left(\mathrm{g} / \mathrm{cm}^{3}\right)\end{array}$ & 3.14 & 2.30 & 2.70 & 2.90 \\
\hline $\begin{array}{c}\text { Fineness } \\
\left(\mathrm{cm}^{2} / \mathrm{g}\right)\end{array}$ & 3555 & 195000 & 4500 & 5970 \\
\hline $\begin{array}{c}\text { Average size } \\
(\mu \mathrm{m})\end{array}$ & 10 & 0.1 & 15 & 45 \\
\hline
\end{tabular}


Table 3.2. Detailed mixture proportion of the pastes.

\begin{tabular}{|lccccccccc|}
\hline Paste ID & w/c & OPC & W & SCM/SP & SP & FA & Slag & SF & \\
& & $(\mathrm{g})$ & $(\mathrm{g})$ & $(\%)$ & $(\mathrm{g})$ & $(\mathrm{g})$ & $(\mathrm{g})$ & $(\mathrm{g})$ & \\
\hline P0.35 & 0.35 & 800 & 280 & 0 & 0 & 0 & 0 & 0 & 36 \\
P0.4 & 0.4 & 700 & 280 & 0 & 0 & 0 & 0 & 0 & 37 \\
P0.45 & 0.45 & 600 & 270 & 0 & 0 & 0 & 0 & 0 & 38 \\
P0.5 & 0.5 & 500 & 250 & 0 & 0 & 0 & 0 & 0 & 39 \\
\hline P0.35-SP0.2 & 0.35 & 800 & 278.40 & 0.2 & 1.60 & 0 & 0 & 0 & 40 \\
P0.4-SP0.2 & 0.4 & 700 & 278.60 & 0.2 & 1.40 & 0 & 0 & 0 & 41 \\
P0.45-SP0.2 & 0.45 & 600 & 268.80 & 0.2 & 1.20 & 0 & 0 & 0 & 42 \\
P0.5-SP0.2 & 0.5 & 500 & 249.00 & 0.2 & 1.00 & 0 & 0 & 0 & 43 \\
\hline P0.3-SP0.5 & 0.3 & 800 & 236.00 & 0.5 & 4.00 & 0 & 0 & 0 & 44 \\
P0.35-SP0.5 & 0.35 & 750 & 258.75 & 0.5 & 3.75 & 0 & 0 & 0 & 45 \\
P0.4-SP0.5 & 0.4 & 700 & 276.50 & 0.5 & 3.50 & 0 & 0 & 0 & 46 \\
P0.45-SP0.5 & 0.45 & 600 & 267.00 & 0.5 & 3.00 & 0 & 0 & 0 & 47 \\
\hline P0.3-SP1.0 & 0.3 & 800 & 232.00 & 1 & 8.00 & 0 & 0 & 0 & 48 \\
P0.35-SP1.0 & 0.35 & 750 & 255.00 & 1 & 7.50 & 0 & 0 & 0 & 49 \\
P0.4-SP1.0 & 0.4 & 700 & 273.00 & 1 & 7.00 & 0 & 0 & 0 & 50 \\
P0.45-SP1.0 & 0.45 & 600 & 264.00 & 1 & 6.00 & 0 & 0 & 0 & 51 \\
\hline P0.35-FA10 & 0.35 & 720 & 280 & 10 & 0 & 80 & 0 & 0 & 56 \\
P0.4-FA10 & 0.4 & 675 & 300 & 10 & 0 & 75 & 0 & 0 & 57 \\
P0.45-FA10 & 0.45 & 630 & 315 & 10 & 0 & 70 & 0 & 0 & 58 \\
P0.5-FA10 & 0.5 & 540 & 300 & 10 & 0 & 60 & 0 & 0 & 59 \\
\hline P0.35-FA30 & 0.3 & 560 & 240 & 30 & 0 & 240 & 0 & 0 & 60 \\
P0.35-FA30 & 0.35 & 525 & 262.5 & 30 & 0 & 225 & 0 & 0 & 61 \\
P0.4-FA30 & 0.4 & 490 & 280 & 30 & 0 & 210 & 0 & 0 & 62 \\
P0.45-FA30 & 0.45 & 420 & 270 & 30 & 0 & 180 & 0 & 0 & 63 \\
P0.5-FA30 & 0.5 & 420 & 300 & 30 & 0 & 180 & 0 & 0 & 64 \\
\hline P0.35-FA50 & 0.3 & 400 & 240 & 50 & 0 & 400 & 0 & 0 & 65 \\
P0.35-FA50 & 0.35 & 375 & 262.5 & 50 & 0 & 375 & 0 & 0 & 66 \\
P0.4-FA50 & 0.4 & 350 & 280 & 50 & 0 & 350 & 0 & 0 & 67 \\
P0.45-FA50 & 0.45 & 300 & 270 & 50 & 0 & 300 & 0 & 0 & 68 \\
P0.5-FA50 & 0.5 & 300 & 300 & 50 & 0 & 300 & 0 & 0 & 69 \\
\hline P0.4-SF5 & 0.4 & 760 & 320 & 5 & 0 & 0 & 0 & 40 & 70 \\
P0.45-SF5 & 0.45 & 712.5 & 337.5 & 5 & 0 & 0 & 0 & 37.5 & 71 \\
P0.5-SF5 & 0.5 & 665 & 350 & 5 & 0 & 0 & 0 & 35 & 72 \\
P0.55-SF5 & 0.55 & 570 & 330 & 5 & 0 & 0 & 0 & 30 & 73 \\
\hline
\end{tabular}


Table 3.2 (Continued). Detailed mixture proportion of the pastes.

\begin{tabular}{|lccccccccc|}
\hline P0.4-SF10 & 0.4 & 720 & 320 & 10 & 0 & 0 & 0 & 80 & 74 \\
P0.45-SF10 & 0.45 & 675 & 337.5 & 10 & 0 & 0 & 0 & 75 & 75 \\
P0.5-SF10 & 0.5 & 630 & 350 & 10 & 0 & 0 & 0 & 70 & 76 \\
P0.55-SF10 & 0.55 & 540 & 330 & 10 & 0 & 0 & 0 & 60 & 77 \\
\hline P0.4-SF15 & 0.4 & 680 & 320 & 15 & 0 & 0 & 0 & 120 & 78 \\
P0.45-SF15 & 0.45 & 637.5 & 337.5 & 15 & 0 & 0 & 0 & 112.5 & 79 \\
P0.5-SF15 & 0.5 & 595 & 350 & 15 & 0 & 0 & 0 & 105 & 80 \\
P0.55-SF15 & 0.55 & 510 & 330 & 15 & 0 & 0 & 0 & 90 & 81 \\
\hline P0.35-SL10 & 0.35 & 720 & 280 & 10 & 0 & 0 & 80 & 0 & 82 \\
P0.4-SL10 & 0.4 & 675 & 300 & 10 & 0 & 0 & 75 & 0 & 83 \\
P0.45-SL10 & 0.45 & 630 & 315 & 10 & 0 & 0 & 70 & 0 & 84 \\
P0.5-SL10 & 0.5 & 540 & 300 & 10 & 0 & 0 & 60 & 0 & 85 \\
\hline P0.3-SL30 & 0.3 & 560 & 240 & 30 & 0 & 0 & 240 & 0 & 86 \\
P0.35-SL30 & 0.35 & 525 & 262.5 & 30 & 0 & 0 & 225 & 0 & 87 \\
P0.4-SL30 & 0.4 & 490 & 280 & 30 & 0 & 0 & 210 & 0 & 88 \\
P0.45-SL30 & 0.45 & 420 & 270 & 30 & 0 & 0 & 180 & 0 & 89 \\
\hline P0.3-SL50 & 0.3 & 400 & 240 & 50 & 0 & 0 & 400 & 0 & 90 \\
P0.35-SL50 & 0.35 & 375 & 262.5 & 50 & 0 & 0 & 375 & 0 & 91 \\
P0.4-SL50 & 0.4 & 350 & 280 & 50 & 0 & 0 & 350 & 0 & 92 \\
P0.45-SL50 & 0.45 & 300 & 270 & 50 & 0 & 0 & 300 & 0 & 93 \\
\hline
\end{tabular}

Weighing of cementitious materials was performed by a balance with $0.1 \mathrm{~g}$ precision, whereas the water and superplasticizer were weighed in $0.01 \mathrm{~g}$ precision. The superplasticizer dosages recommended by the supplier range from $0.2 \%$ to $1.1 \%$ of cement mass. In order to cover the entire suggested range of application, addition levels of $0.2 \%, 0.5 \%$ and $1.0 \%$ were selected in this study, corresponding to low, medium and high superplasticizer dosages, respectively. In the specimen labeling convention, the pastes containing superplasticizer were denoted by SP followed by dosage percentage (e.g. SP 0.2 , which stands for paste with $0.2 \%$ (by cement mass) of superplasticizer).

For each SCM, such as fly ash, silica fume and slag, three different dosages were used in the experiments to cover the whole range of application from low, medium and high 
dosages. Their dosage was defined by replacement in the total cementitious materials content; i.e., the percentage of SCM weight to the weight of cement plus SCM (i.e., total cementitious material content). Fly ash and slag were added at $10 \%(11.4 \%$ and $10.7 \%$ by volume for fly ash and slag, respectively), $30 \%$ (33.3\% and $31.7 \%$ by volume for fly ash and slag, respectively) and 50\% (53.8\% and $52.0 \%$ by volume for fly ash and slag, respectively) replacement levels, while silica fume was used at $5 \%$ ( $6.70 \%$ by volume), $10 \%(13.2 \%$ by volume $)$ and $15 \%$ (19.4\% by volume) replacement levels. In the labeling convention used in this study, the paste which is labeled as "FA30" corresponds to a paste containing $30 \%$ of fly ash.

The water-to-cementitious materials ratio $(\mathrm{w} / \mathrm{c})$ of the mixtures ranged from 0.3 to 0.55 with 0.05 increments to cover a wide range of possible w/c ratios used in the construction industry. In the labeling convention, the $\mathrm{w} / \mathrm{c}$ is also designated; for instance, "P0.45SF15" identifies a paste containing $15 \%$ of silica fume ( $85 \%$ of OPC) with the w/c of 0.45 .

\subsubsection{Preparation of fresh paste}

Before mixing the materials, the mold and electrodes, conductive rods, which were in constant contact with the fresh paste during experiments were rinsed with distilled water and dried by delicate task wipers to make sure that no physical or electrical contamination was left on them. The bowl and blender used to batch the materials were made of stainless steel to make sure that no static electricity would be generated. The exact time of adding water, and subsequently mixing time, was recorded. Blending of mixture continued until homogeneous paste was obtained after about 4 minutes (see Fig. 
3.1). The fixed volume of paste was then poured into the mold in a way that the height of fresh paste in the mold was constant for all of the mixtures within $\pm 1.0 \mathrm{~mm}$.

\subsubsection{Storage of hardened paste}

In order to test the pore solution of hardened cement paste, fresh cement paste samples were collected from each mixture after the electrical resistivity test and these samples were stored in sealed plastic bags so that the hardening of the cement paste occurred under sealed curing conditions, as shown in Fig. 3.2. This way, the loss of water content of the cement pastes during setting and hardening of cement was minimized. Fourteen selected hardened samples (i.e., P0.35, P0.5, P0.35-SP1.0, P0.45-SP1.0, P0.35-FA30, P0.5-FA30, P0.45-SF5, P0.55-SF5, P0.45-SF10, P0.55-SF10, P0.35-SL10, P0.45-SL10, P0.35-SL30, and P0.45-SL30) were used for pore solution extraction and chemical analysis of pore solution after setting to cover all five main groups of our paste mixtures, the effect of w/c change, as well as the variation in the SCM dosage.

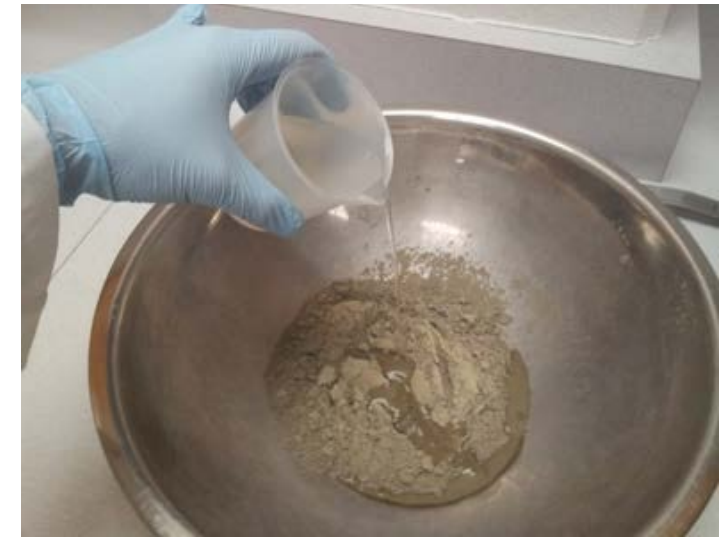

(a)

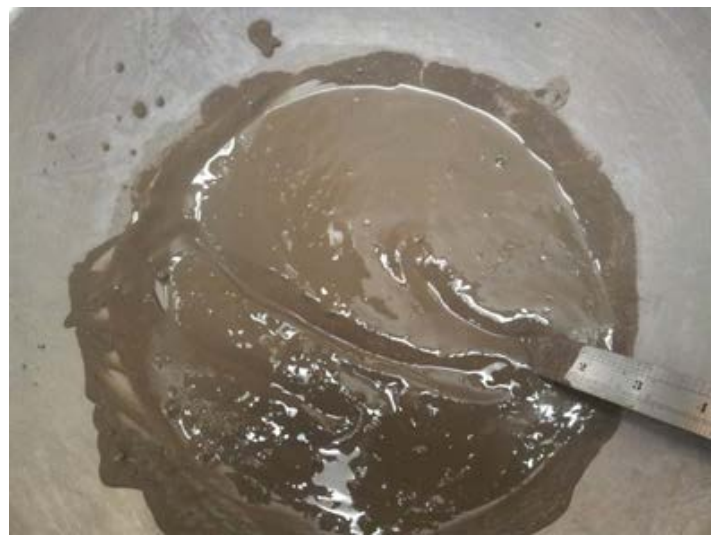

(b)

Figure 3.1. Fresh paste preparation: a) water addition to cementitious materials; b) homogenous paste after mixing. 


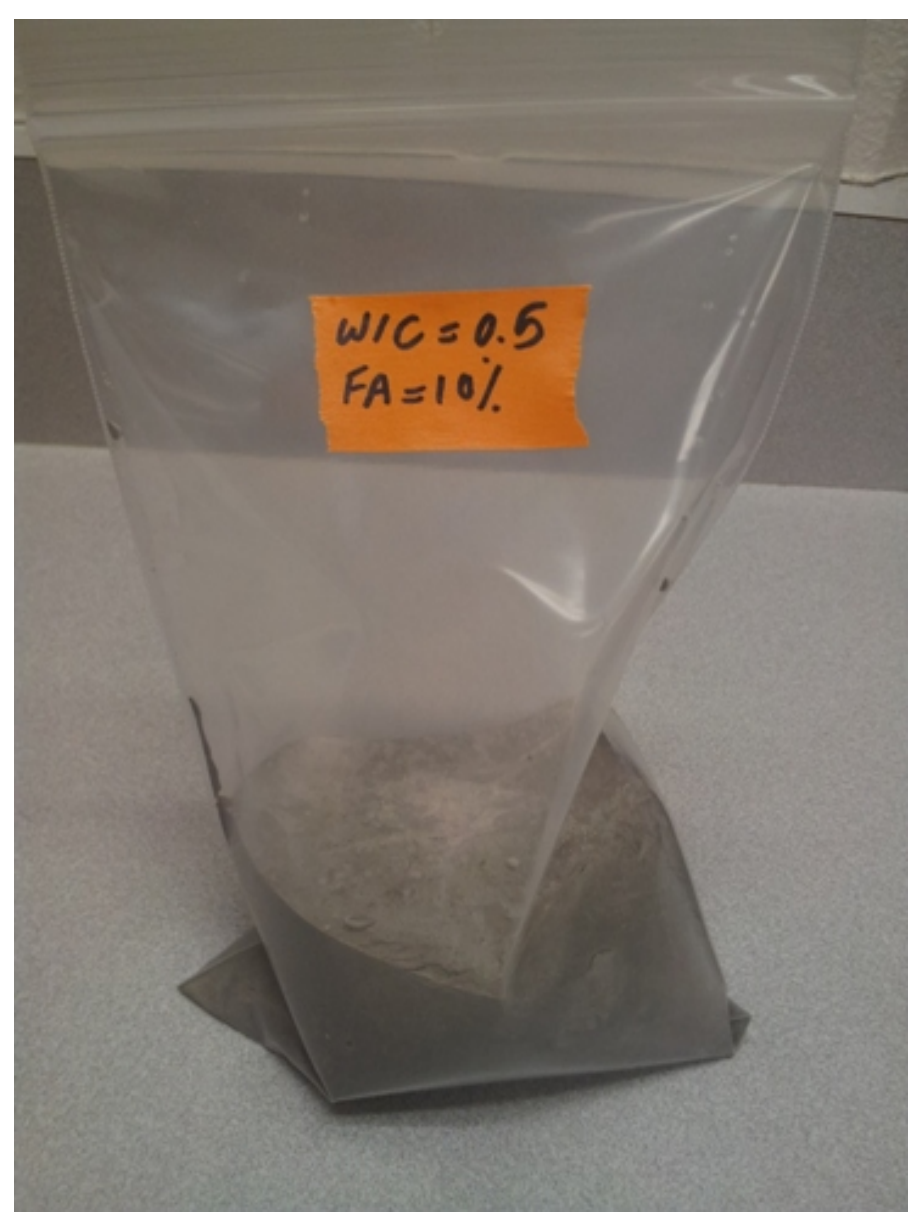

Figure 3.2. Collection of hardened paste samples in a sealed curing condition.

\subsubsection{Solution extraction from fresh paste}

About $400 \mathrm{~g}$ of the fresh paste from each batch was collected for solution extraction. The liquid phase of the fresh paste can be separated out using different methods such as employing a centrifuge or vacuum pump. In this research, a GAST vacuum pump with a maximum vacuum pressure of $760 \mathrm{mmHg}$ was used to apply suction. Also an Erlenmeyer flask, funnel, sealing rubber and 3 layers of Whatman ${ }^{\mathrm{TM}}$ filter papers (No 1001-185, 20$25 \mu \mathrm{m})$ were used in the setup shown in Fig. 3.3 to perform the extraction process. 


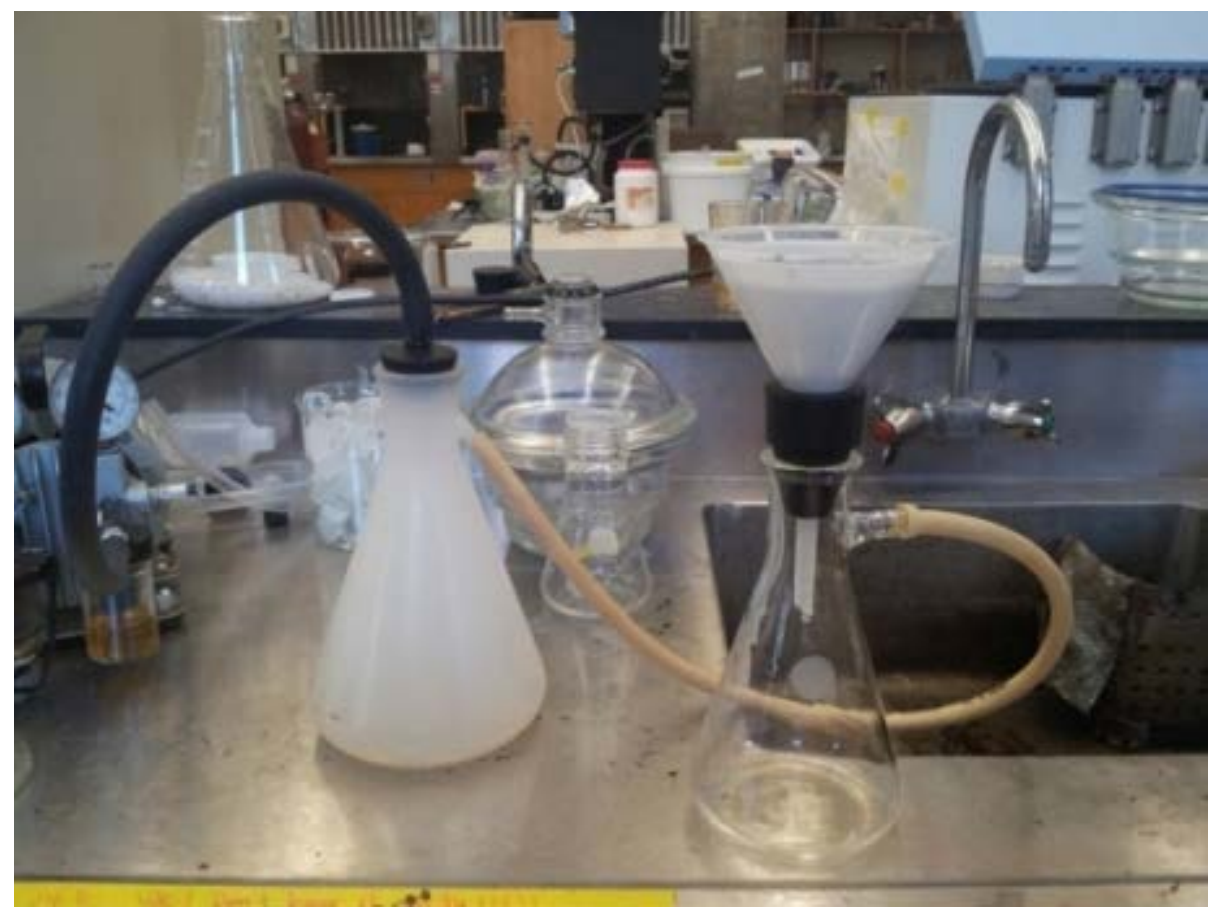

(a)

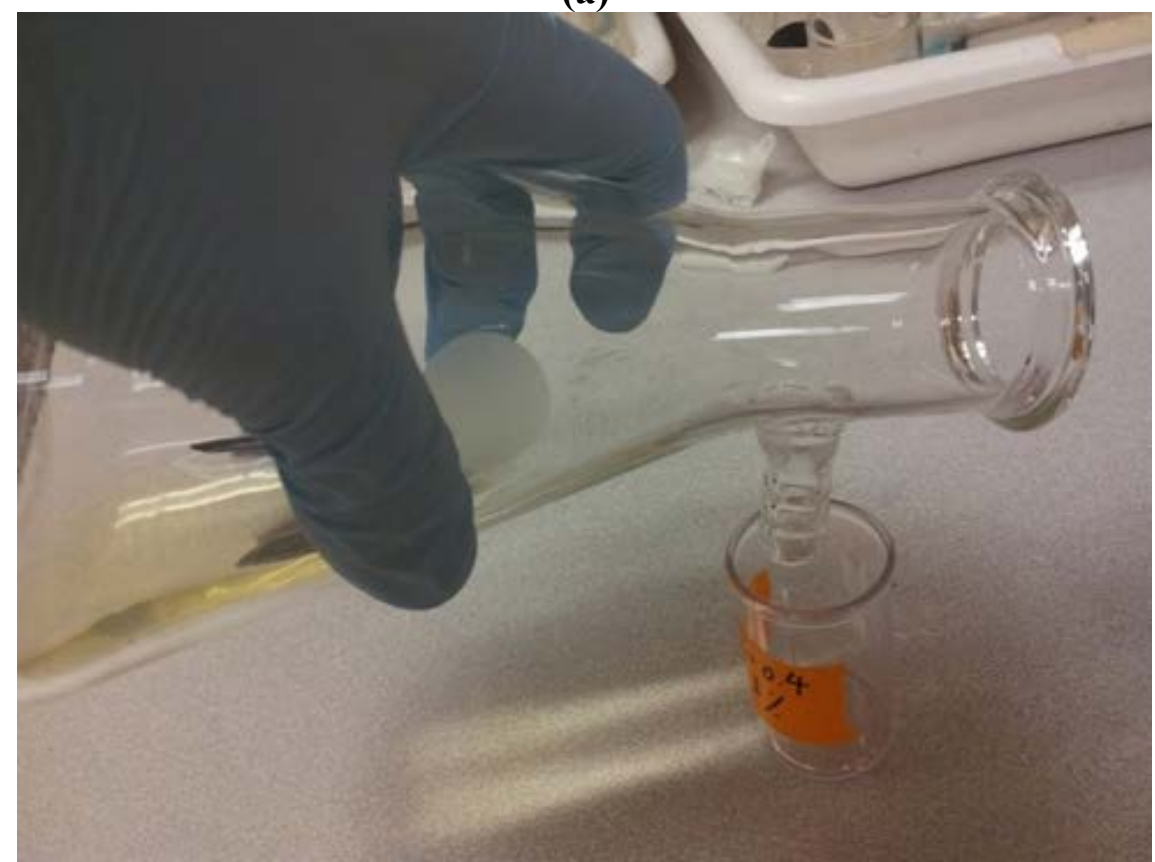

(b)

Figure 3.3. Solution extraction process from fresh paste: a) test setup to apply suction through vacuum pump; b) sample collection. 
Before each extraction, the Erlenmeyer flask and funnel were completely rinsed and dried to make sure that there was no trace of solution or distilled water from the previous extraction. The extraction process was conducted at the $30^{\text {th }}, 60^{\text {th }}$ and $90^{\text {th }}$ minute after mixing the materials, and it took an average of 6 minutes to collect about $20 \mathrm{ml}$ of solution. The 6 minutes was the average time because the extraction time was highly dependent on the $\mathrm{w} / \mathrm{c}$ of the mixture. When the extraction process was completed, the extracted solution was collected in plastic tubes with air-tight lids which had been labeled specifying the type of cement paste mixture (see Fig. 3.3).

\subsubsection{Solution extraction from hardened paste}

The pore solution of the hardened pastes, at about 5 months age, was extracted under high pressure using a pore press, which consists of several specially machined pieces of steel that are placed in an ordinary universal testing machine for purposes of pore solution extraction. Schematic of a typical pore press is shown in Fig. 3.4, which illustrates three main pieces labeled Parts A to C. Each hardened paste sample was crushed and placed in the dashed lined area in the interior of Part A. The applied load for the paste samples were between 100 and 150 kips in order to obtain about $2 \mathrm{ml}$ of pore solution in the designated volume in Part B. Eventually, expressed pore solution was captured by a syringe attached to the Part B and collected in plastic tubes with air-tight lids. 


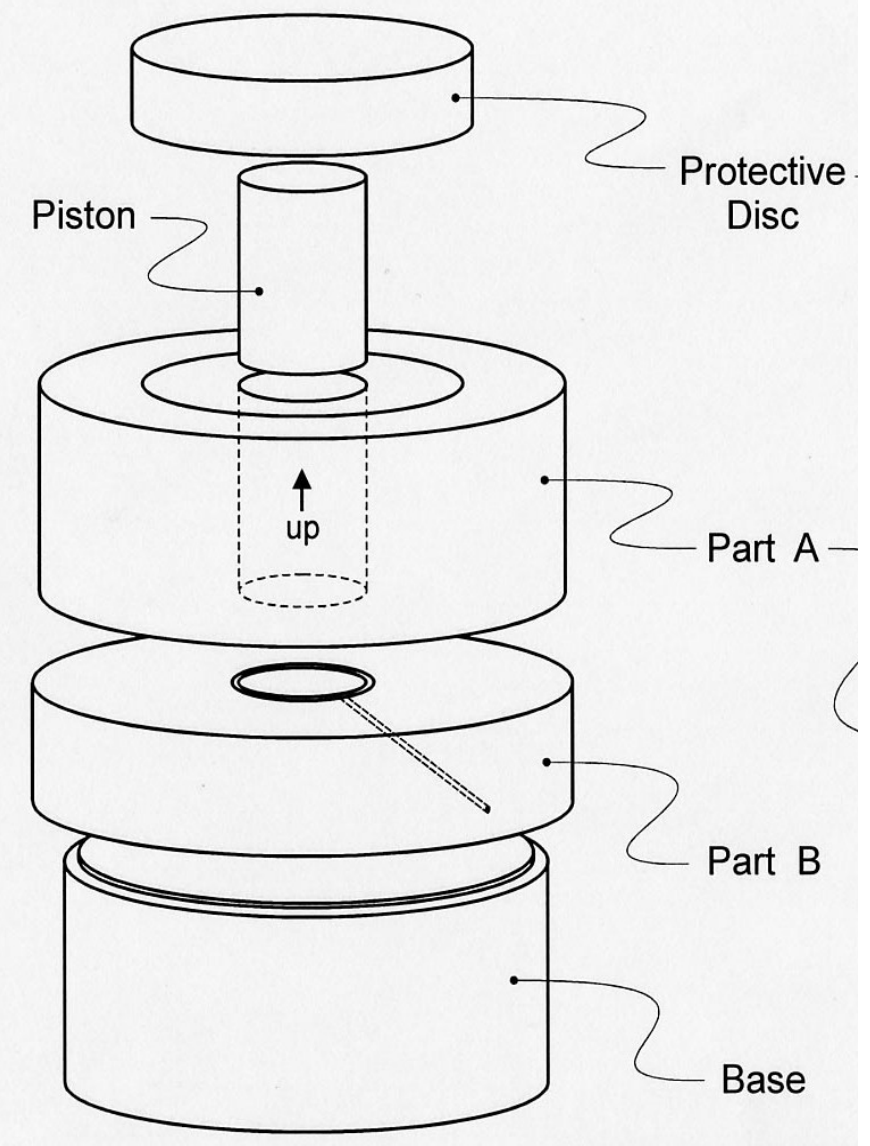

Figure 3.4. Schematic shape of pore press (source: Benoit Fournier).

\subsection{Test Setup}

All the tests conducted on the samples can be classified in three main categories: (1) paste electrical resistivity test, (2) pore solution conductivity test, and (3) $\mathrm{pH}$ measurements, which are described in the following sections.

\subsubsection{Impedance spectroscopy}

The method used to measure the electrical resistivity of fresh paste is called impedance spectroscopy. In this study, impedance spectroscopy test was performed such that a 
concrete impedance measurement instrument was used to apply A.C. currents between two electrodes inside the sample (see Fig. 3.5) with sweeping frequencies from $1 \mathrm{~Hz}$ to $30 \mathrm{kHz}$ with logarithmic increments at 6 second intervals and measures the corresponding phase angle as well as drop in voltage, impedance. The choice of the frequency range was made based on the preliminary experiments, which are presented in Section 5.2.1. This way the resistance of cement paste material which corresponds to minimum phase angle values was obtained. In this study the resistance of cement paste was measured every four minutes after mixing for 2 hours in the room temperature (around $21^{\circ} \mathrm{C}$ ). The internal temperature of paste was being also recorded to compensate the effect of temperature on the electrical resistivity of the paste. The temperature rise in the cement paste was caused by the heat release during the hydration of cement paste materials. This resulted in maximum of $4{ }^{\circ} \mathrm{C}$ increase in temperature after mixing the materials. All the values were normalized to a reference temperature of $25^{\circ} \mathrm{C}$. The normalization process is explained in Chapter 5. In this study, Giatec $\mathrm{RCON}^{\mathrm{TM}}$ [59] device was used for performing impedance measurements.

Fresh paste samples were poured into plastic cylinder molds with $75 \mathrm{~mm}$ diameter. The height of fresh paste in the mold was adjusted to $50 \mathrm{~mm}$. The stainless steel electrodes were inserted into the paste and fixed to the top part of the mold with a plastic space holder to keep them parallel in upright position (Fig. 3.5). The distance between the rods was kept at $50 \mathrm{~mm}$ for all the tests. The electrical current only flowed through the $10 \mathrm{~mm}$ exposed area of the electrodes. As shown in Fig. 3.5, other areas of the electrodes were covered by shrink tube from top and epoxy coating from bottom to limit the passage of current only through the exposed areas. Temperature sensor was placed in the paste 83 
outside the area between the electrodes to minimize the current flow distortion. Two alligator clips were then attached to the top end side of the electrodes and measurements started at around 7 minutes after mixing. Fig. 3.6 shows the test setup explained above while recording the data.

In order to obtain the geometry factor of this test setup, a standard $\mathrm{NaCl}$ solution with a known conductivity of $20.0 \mathrm{mS} / \mathrm{cm}$ (resistivity $=0.5 \Omega . \mathrm{m}$ ) at $25^{\circ} \mathrm{C}$ was used. The selection of the type of the known conductivity solution was based on our initial knowledge on the range of the electrical resistivity of fresh cement pastes. Having the resistivity, $\rho$, of standard solution $(0.5 \Omega$.m) and corresponding resistance $\mathrm{R}(\Omega)$ taken from RCON, the geometry factor of our test setup was calculated via:

$$
G_{F}=\frac{R_{S T}}{\rho_{S T}}
$$

where $\mathrm{G}_{\mathrm{F}}$ is geometry factor in $\mathrm{m}^{-1}$; and $\mathrm{R}_{\mathrm{ST}}$ and $\rho_{\mathrm{ST}}$ are the resistance and resistivity of the standard solution, respectively.

The consistency of the geometry factor was monitored before starting the test to ensure that no electrical leakage occurred from insulating parts of the rods and also the consistency in the geometrical configuration of the test setup such as the distance between the rods, their positions in the mold and the height of the rods. Figure 3.5 schematically shows the details of the electrodes in the test setup. 


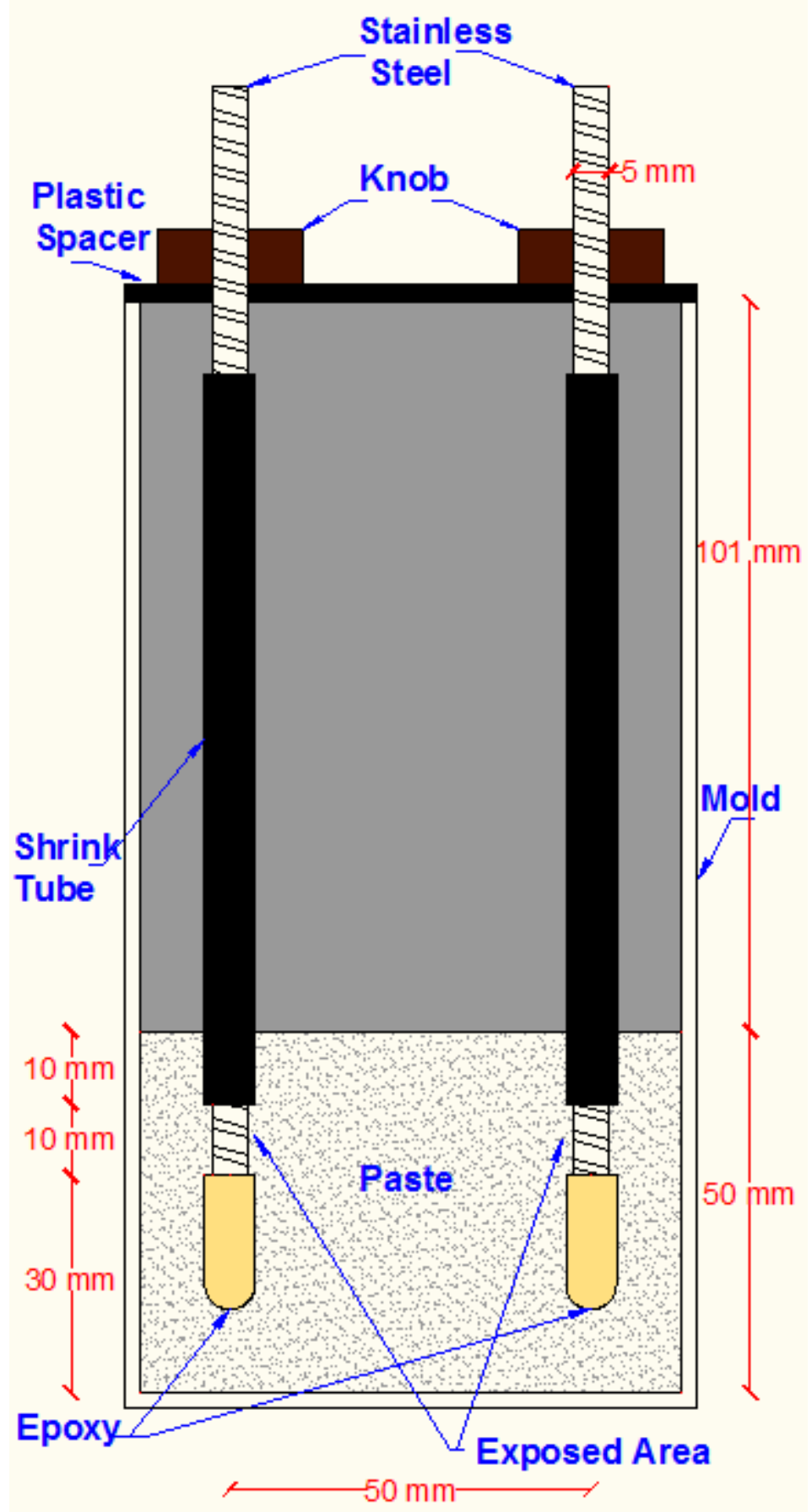

Figure 3.5. Section of test setup for electrical resistivity measurement. 


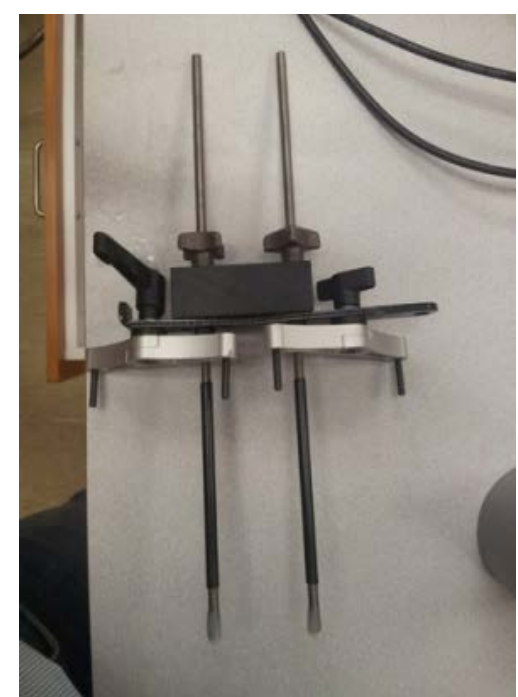

(a)

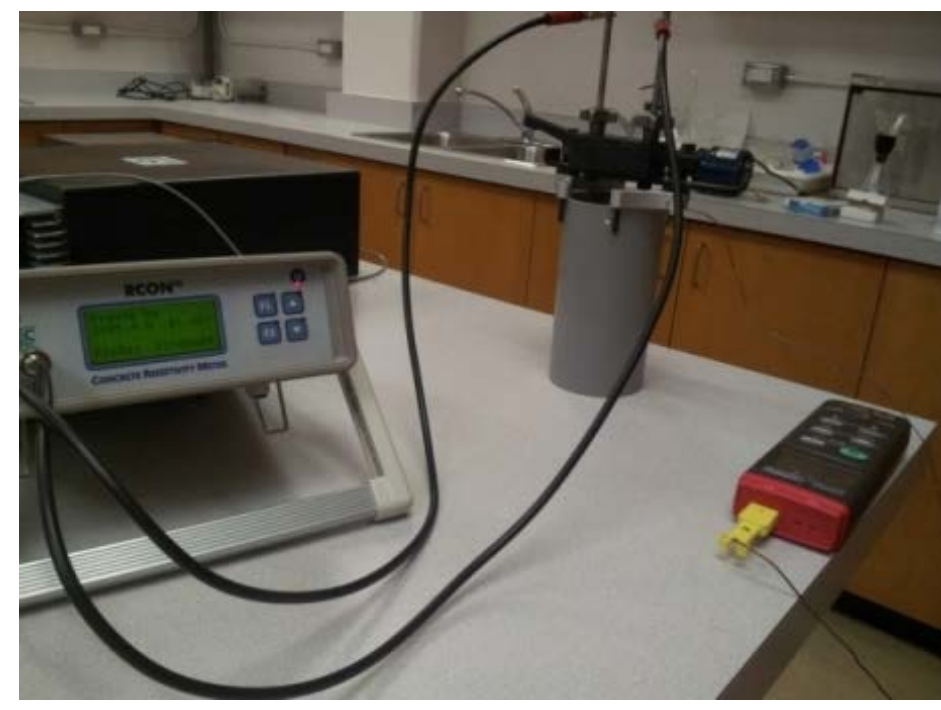

(b)

Figure 3.6. Paste sample resistivity measurement: a) electrodes anchored with spacer to keep them in parallel position; b) test setup for electrical resistivity measurement with RCON and temperature monitoring.

\subsubsection{Solution conductivity measurements}

The conductivity measurement was conducted on solutions extracted from fresh paste at different hydration ages of $30^{\text {th }}, 60^{\text {th }}$ and $90^{\text {th }}$ minute as well as solutions squeezed out from hardened pastes at the hydration age of around 5 months using SymPHony SP90M5 with a temperature sensor for temperature correction. The conductivity probe/cell and the instrument were calibrated to directly give the normalized conductivity of pore solutions at reference temperature of $25^{\circ} \mathrm{C}$, while corresponding temperature also was recorded. In the calibration procedure, standard solution of $\mathrm{NaCl}$ with $20.0 \mathrm{mS} / \mathrm{cm}$ was employed to set cell coefficient of conductivity probe as well as linear temperature compensation $\alpha$. Initially, at $25^{\circ} \mathrm{C}$ the cell coefficient was set in a way that the instrument provided the exact value of $20 \mathrm{mS} / \mathrm{cm}$. Then, $\alpha$ was found by changing the temperature of one solution 
while the instrument gives the constant value for $25^{\circ} \mathrm{C}$ conductivity. Accordingly, the temperature compensation factor of $0.013{ }^{\circ} \mathrm{C}^{-1}$ was obtained.

Each measurement was conducted after rinsing the conductivity cell and drying it to make sure that no contamination had been left from the previous measurement. In order to make sure that measurement was taken conveniently without any movement, a benchtop probe fixture with a holder was used as shown in Fig. 3.7.

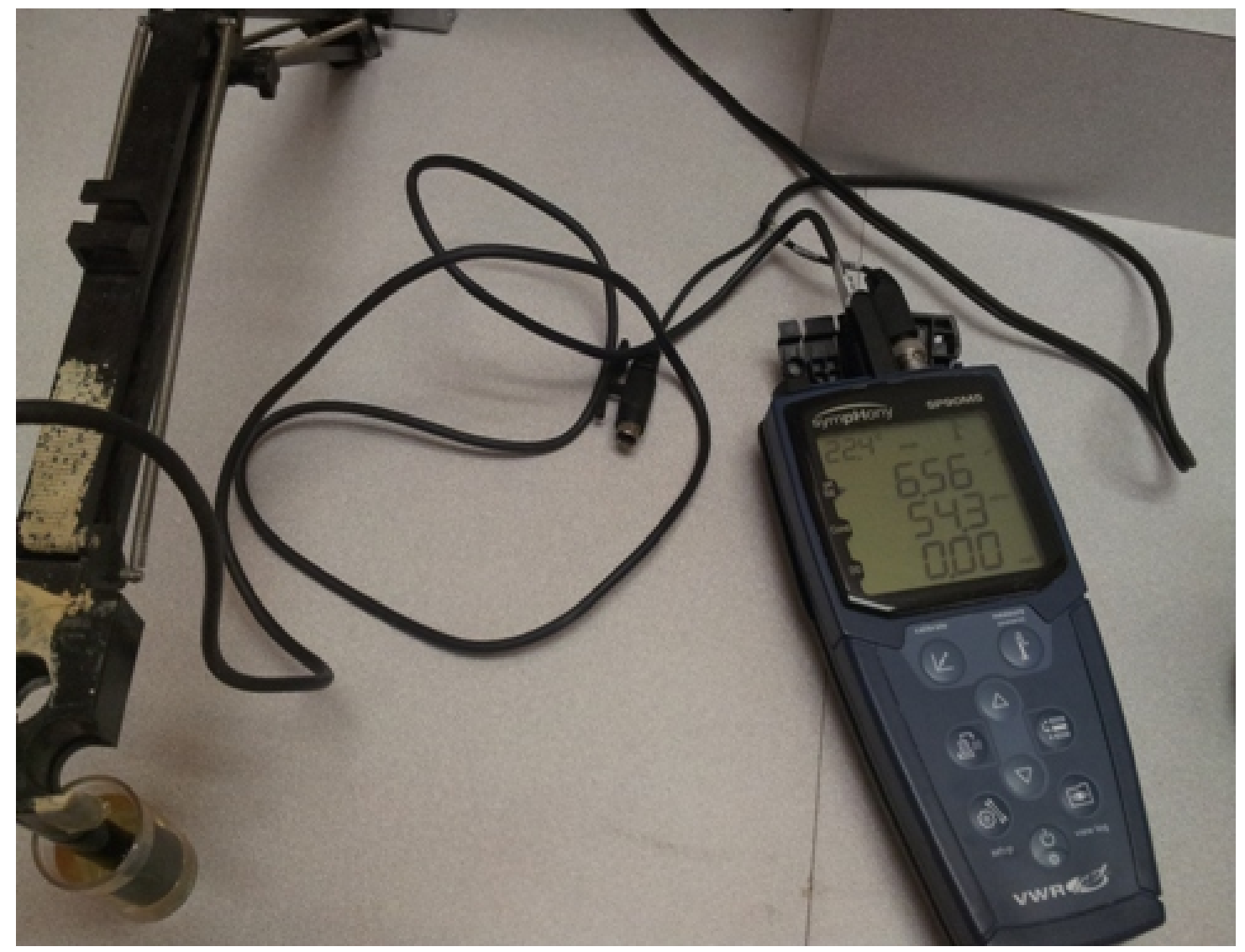

Figure 3.7. Conductivity measurement test for pore solution. 
The pore solution quantities, which were extracted from four out of fourteen hardened pastes (i.e., P0.35, P0.5, P0.35-SP1.0 and P0.45-SP1.0 samples) after about 5 months, were not enough to directly measure their conductivity with the instrument. Because the depth of the pore solution in plastic tube could not cover the entire volume of the conductivity cell, the instrument consistently under measured the conductivity. Thus, pore solutions extracted from hardened pastes were diluted with known volume of distilled water to indirectly calculate their conductivity. In a dilution process in which the number of ions is constant, we can estimate conductivity of a pore solution before dilution $\left(\sigma_{1}\right)$ if the corresponding conductivity of diluted pore solution $\left(\sigma_{2}\right)$ is known using Eq. 3.2. From Eq. 2.8 it is assumed that conductivity is directly proportional with square root of ions concentration via:

$\frac{\sigma_{1}}{\sigma_{2}}=\sqrt{\frac{C_{1}}{C_{2}}}=\sqrt{\frac{V_{2}}{V_{1}}}$

where $\mathrm{C}_{1}$ and $\mathrm{C}_{2}$ are ions concentration ( $\left.\mathrm{mol} / \mathrm{l}\right)$ before and after dilution, respectively; and $\mathrm{V}_{1}$ and $\mathrm{V}_{2}$ are solutions volume (l) before and after dilution, respectively.

\subsection{3. $\mathrm{pH}$ measurements}

The instrument used to conduct the $\mathrm{pH}$ measurements was also symPHony SP90M5, the same instrument employed for conductivity measurements. For the calibration, three buffer solutions of $\mathrm{pH} 10.00,12.00$ and 13.00 were used at $25^{\circ} \mathrm{C}$, because they represent the range of $\mathrm{pH}$ of cement pastes and their pore solution that are investigated in this study. 


\subsubsection{Paste measurements}

Since the $\mathrm{pH}$ of the paste was time dependant as a result of ion dissolutions of various phases of cement, the measurements were conducted at $30^{\text {th }}, 60^{\text {th }}$ and $90^{\text {th }}$ minutes after the completion of mixing the cementitious materials with water. After rinsing and drying to avoid the effect of contamination, the tip of the $\mathrm{pH}$ electrode was inserted into the paste. When the $\mathrm{pH}$ reading on the meter became stable, the $\mathrm{pH}$ value and associated temperature were recorded. The temperatures were used to normalize the $\mathrm{pH}$ readings to the reference temperature of $25{ }^{\circ} \mathrm{C}$ using a temperature compensation factor. Based on the experimental data we collected on the buffer solutions the temperature compensation factor of $0.01{ }^{\circ} \mathrm{C}^{-1}$ for $\mathrm{pH}$ corrections was used (Fig. 3.8).

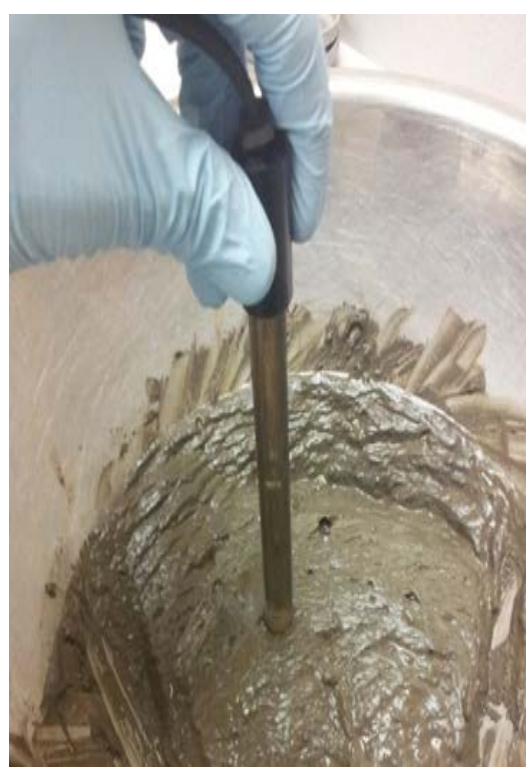

(a)

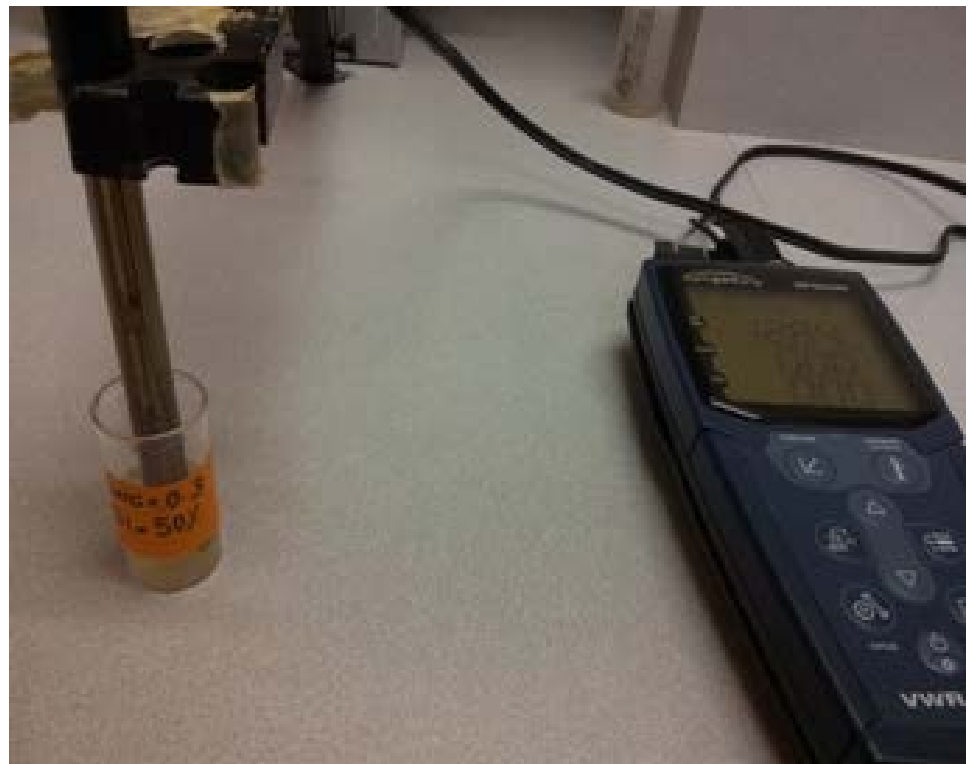

(b)

Figure 3.8. $\mathrm{pH}$ measurement test for: a) paste; b) pore solution. 


\subsubsection{Pore solution measurements}

The $\mathrm{pH}$ of the pore solution samples extracted at specific times of $30^{\text {th }}, 60^{\text {th }}$ and $90^{\text {th }}$ minutes after mixing was measured using the same $\mathrm{pH}$ meter. An electrode holder was also used to hold the $\mathrm{pH}$ probe/electrode in the solution, as shown in Fig. 3.8. After stabilization of the $\mathrm{pH}$ value, the $\mathrm{pH}$ value and temperature were recorded and were adjusted to the reference temperature of $25{ }^{\circ} \mathrm{C}$ using a temperature compensation factor of $0.01{ }^{\circ} \mathrm{C}^{-1}$. The $\mathrm{pH}$ of the pore solution for four hardened pastes, for which the volume was too low to completely cover the electrode, was indirectly calculated using the $\mathrm{pH}$ of the diluted solution as follows:

$p H_{1}=p H_{2}-\log \left(\frac{V_{1}}{V_{2}}\right)$

where $\mathrm{pH}_{1}$ and $\mathrm{pH}_{2}$ are the $\mathrm{pH}$ of pore solution sample and diluted solution, respectively; and $V_{1}$ and $V_{2}$ are the know volume of pore solution sample and diluted solution, respectively. 


\section{Results and Discussion}

\subsection{Introduction}

The fresh paste has two components including conductive pore solution (liquid phase) and nonconductive solid particles (solid phase). The solid phase also consists of cement particles and supplementary cementitious materials. The liquid phase is mainly composed of water, but in some cases, might also include small amounts of superplasticizer or water-reducer admixtures. The relationship between electrical resistivity of the paste (i.e., pore solution plus solid phase) and its pore solution can also be defined using Archie's law [19] through formation factor, F. The possibility of using this equation to obtain the electrical resistivity/conductivity of paste from that of the pore solution was investigated in this chapter. It should be noted that following section only includes the typical representative results (i.e., selected results) and the interpreted results are presented as part of the discussion section (i.e., section 4.3). The supplementary test results can be found in Appendix B.

\subsection{Selected Results}

\subsubsection{Paste}

As mentioned in Section 3.4.1, the instrument that was used to measure the electrical resistivity of the paste, Giatec $\mathrm{RCON}^{\mathrm{TM}}$ [59], measures the impedance (Z) rather than the electrical resistance. In order to convert the impedance values to resistance (R), the capacitance effect should be eliminated, which occurs when the phase angle $(\varphi)$ between the current and voltage is zero, as in the case of a pure resistor. In order to determine the 
resistance of fresh paste, a preliminary study was conducted to determine the best frequency which corresponds to the lowest phase angle. In this study the frequency of the measurements were varied from 1 to $30,000 \mathrm{~Hz}$. Figures 4.1 and 4.2 show the variation of impedance and phase angle, respectively, with frequency. Each sweeping cycle took around 3 minutes in $\mathrm{RCON}^{\mathrm{TM}}$ for a typical paste $(\mathrm{OPC}, \mathrm{w} / \mathrm{c}=0.45)$. The impedance spectra were recorded during the first two hours after mixing the materials. Fig. 4.1 shows that the rate of change in impedance after $1000 \mathrm{kHz}$ is negligible. In addition, as shown in Fig. 4.2, the phase angle decreases with increasing frequency. Therefore, the frequency of $30 \mathrm{kHz}$ was chosen in this study to measure the electrical resistivity of fresh pastes. At this frequency, the phase angle of the measurements was around 4 degrees.

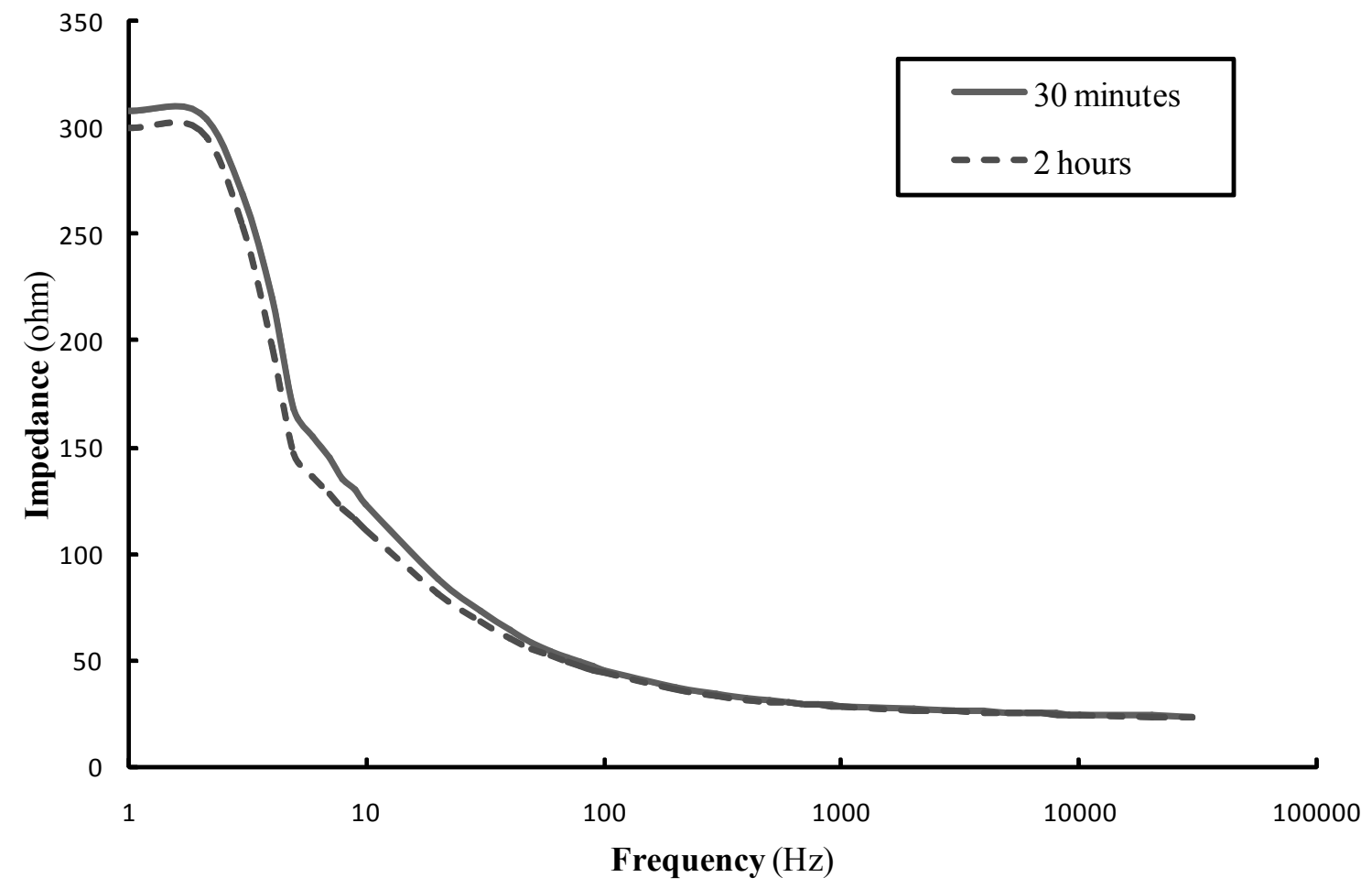

Figure 4.1. Impedance-frequency spectra in one cycle of frequency sweep. Although measurements were taken at different times, for clarity, data from only two sweeps (at 30 minutes and 2 hours) are shown. 


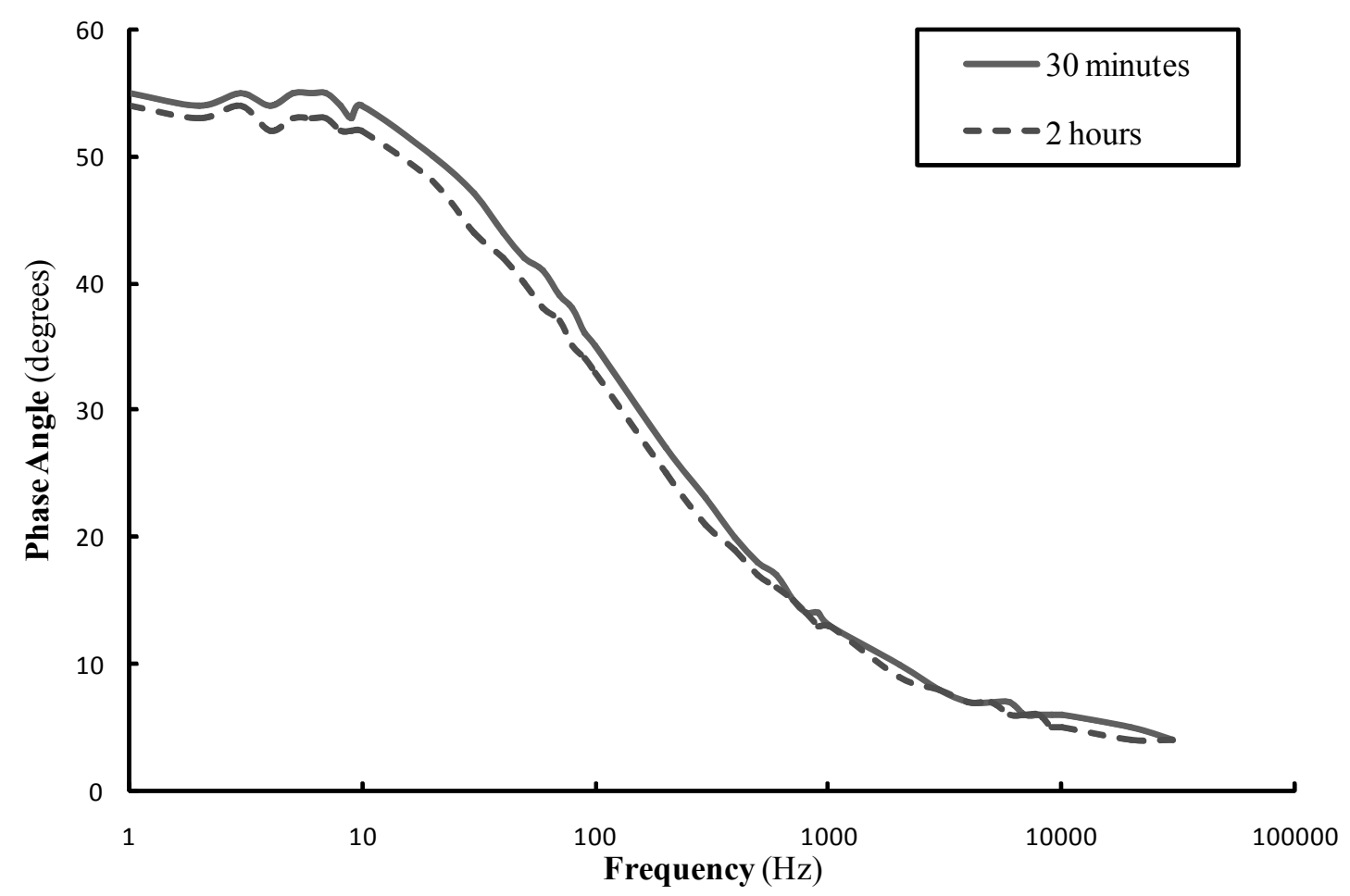

Figure 4.2. Phase angle-frequency spectra in one cycle of frequency sweep. Although measurements were taken at different times, for clarity, data from only two sweeps (at 30 minutes and 2 hours) are shown.

The geometry of the test setup affects the resistance measurement (R), while the electrical resistivity of the fresh paste $(\rho)$ is independent from the geometry and is related to the property of the cement paste. The geometry factor $\left(\mathrm{G}_{\mathrm{F}}\right)$ of our test setup, which is shown in Fig. 3.5, was determined by using the standard $\mathrm{NaCl}$ solution with $20.0 \mathrm{mS} / \mathrm{cm}$ conductivity at $25^{\circ} \mathrm{C}$. The linear compensation factor $\alpha$ was $0.013^{\circ} \mathrm{C}^{-1}$. Temperature of standard solution $(\mathrm{T})$ and corresponding resistance $\left(\mathrm{R}_{\mathrm{ST}}\right)$ were recorded by thermometer and Giatec $\mathrm{RCON}^{\mathrm{TM}}$, respectively. The linear relationship for electrolytic solutions (Eq. 4.1) was then used to find the resistivity of standard solution at temperature $T$ as follows: 
$\sigma_{S T}=\sigma_{\theta}[1+\alpha \cdot(T-\theta)]$

$\rho_{S T}=\frac{1}{\sigma_{S T}}$

where $\sigma_{\mathrm{ST}}$ and $\sigma_{\theta}$ are the electrical conductivity $(\mathrm{S} / \mathrm{m})$ of standard solution at temperature $\mathrm{T}$ and reference temperature $\theta$, respectively; and $\rho_{\mathrm{T}}$ is the electrical resistivity $(\Omega . \mathrm{m})$ of standard solution at temperature $\mathrm{T}$. The geometry factor $\left(\mathrm{G}_{\mathrm{F}}\right)$ in $\mathrm{m}^{-1}$ can then be expressed via Eq. 3.1. The geometry factor was calculated every time before starting the tests to make sure that there was no significant change. When there was a change, the updated value was used to calculate the resistivity from the resistance. The geometry factor of our test setup was $54.6 \pm 0.2 \mathrm{~m}^{-1}$.

Since the internal temperature of paste is variable during the first two hours after mixing, the measured resistivity values were normalized to a reference temperature of $25^{\circ} \mathrm{C}$. Assuming resistivity changes linearly with temperature [42, 43], and assuming the same temperature compensation factor $\alpha$ of $0.013^{\circ} \mathrm{C}^{-1}$ similar to that of pore solutions, the normalization coefficient to reference temperature of $25^{\circ} \mathrm{C}$ from paste internal temperature was calculated through:

$$
\begin{aligned}
& \rho_{25}=C_{25} \times \rho_{T} \\
& C_{25}=1-0.013(25-T)
\end{aligned}
$$

where $C_{25}$ is the normalization coefficient of resistivity to $25{ }^{\circ} \mathrm{C}$; and $\rho_{25}$ and $\rho_{\mathrm{T}}$ are the paste electrical resistivities at $25^{\circ} \mathrm{C}$ and at temperature $\mathrm{T}$, respectively. 
The $\mathrm{pH}$ of the paste and its temperature was measured by a $\mathrm{pH}$ meter, symPHony SP90M5, and normalized to the reference temperature of $25^{\circ} \mathrm{C}$. The range of temperature variation in the cement pastes during the measurements was limited to $4{ }^{\circ} \mathrm{C}$. The variation of $\mathrm{pH}$ with temperature observed experimentally in this range $\left(4{ }^{\circ} \mathrm{C}\right)$ was almost linear. In the $\mathrm{pH}$ range of our pastes (around 13), increasing the temperature by $1{ }^{\circ} \mathrm{C}$ decreased the $\mathrm{pH}$ values by 0.01 . Therefore, the temperature compensation factor $\alpha$ was set to $-0.01 \mathrm{pH} /{ }^{\circ} \mathrm{C}$ to normalize the values given by the $\mathrm{pH}$ meter to the reference temperature of $25{ }^{\circ} \mathrm{C}$, which was in agreement with the provided temperature compensation for buffer solutions.

The typical results of resistivity and $\mathrm{pH}$ tests are presented in Table 4.1 and 4.2 for OPC plus $30 \%$ fly ash and OPC plus $0.5 \%$ superplasticizer, respectively. Since the resistivity and $\mathrm{pH}$ values are time dependent, the measurements were taken at $30^{\text {th }}$ minute of hydration age to have comparable test results and eliminate the effect of time. Further details are discussed in Section 4.3.1.

Table 4.1. Paste resistivity and $\mathrm{pH}$ results for OPC plus $30 \%$ fly ash at $30^{\text {th }}$ minute after mixing.

\begin{tabular}{|lcccccccc|}
\hline \multicolumn{1}{|c}{ Paste ID } & $\mathbf{w} / \mathbf{c}$ & $\begin{array}{c}\mathbf{R} \\
(\Omega)\end{array}$ & $\begin{array}{c}\mathbf{G}_{\mathbf{F}} \\
\left(\mathrm{m}^{-1}\right)\end{array}$ & $\begin{array}{c}\boldsymbol{\rho}_{\mathbf{T}} \text { paste } \\
(\Omega . \mathrm{m})\end{array}$ & $\begin{array}{c}\mathbf{T}_{\text {paste }} \\
\left({ }^{\circ} \mathrm{C}\right)\end{array}$ & $\begin{array}{c}\mathbf{C}_{25} \\
\boldsymbol{\rho}_{25 \text { paste }} \\
(\Omega . \mathrm{m})\end{array}$ & $\begin{array}{c}\mathbf{p H}_{25} \\
\text { paste }\end{array}$ \\
\hline P0.35-FA30 & 0.3 & 31.4 & 54.6 & 0.5751 & 24.9 & 0.9987 & 0.5743 & 13.33 \\
P0.35-FA30 & 0.35 & 29 & 54.6 & 0.5311 & 24.5 & 0.9935 & 0.5277 & 13.33 \\
P0.4-FA30 & 0.4 & 29 & 54.6 & 0.5311 & 24.3 & 0.9909 & 0.5263 & 13.29 \\
P0.45-FA30 & 0.45 & 29.1 & 54.6 & 0.5330 & 24.1 & 0.9883 & 0.5267 & 13.25 \\
P0.5-FA30 & 0.5 & 29.2 & 54.6 & 0.5348 & 23.8 & 0.9844 & 0.5265 & 13.22 \\
\hline
\end{tabular}


Table 4.2. Paste resistivity and $\mathrm{pH}$ results for OPC plus $0.5 \%$ superplasticizer at $30^{\text {th }}$ minute after mixing.

\begin{tabular}{|lcccccccc|}
\hline \multicolumn{1}{|c}{ Paste ID } & $\mathbf{w} / \mathbf{c}$ & $\begin{array}{c}\mathbf{R} \\
(\Omega)\end{array}$ & $\begin{array}{c}\mathbf{G}_{\mathbf{F}} \\
\left(\mathrm{m}^{-1}\right)\end{array}$ & $\begin{array}{c}\boldsymbol{\rho}_{\text {T paste }} \\
(\Omega . \mathrm{m})\end{array}$ & $\begin{array}{c}\mathbf{T}_{\text {paste }} \\
\left({ }^{\circ} \mathrm{C}\right)\end{array}$ & $\mathbf{C}_{\mathbf{2 5}}$ & $\begin{array}{c}\boldsymbol{\rho}_{25} \text { paste } \\
(\Omega . \mathrm{m})\end{array}$ & $\begin{array}{c}\mathbf{p H}_{\mathbf{2 5}} \\
\text { paste }\end{array}$ \\
\hline P0.3-SP0.5 & 0.3 & 27.7 & 54.6 & 0.5073 & 26.1 & 1.0143 & 0.5146 & 13.40 \\
P0.35-SP0.5 & 0.35 & 24.9 & 54.6 & 0.4560 & 25.8 & 1.0104 & 0.4608 & 13.37 \\
P0.4-SP0.5 & 0.4 & 23.9 & 54.6 & 0.4377 & 25.3 & 1.0039 & 0.4394 & 13.33 \\
P0.45-SP0.5 & 0.45 & 23.6 & 54.6 & 0.4322 & 24.9 & 0.9987 & 0.4317 & 13.32 \\
\hline
\end{tabular}

\subsubsection{Pore solution}

The measurements of pore solution conductivity and $\mathrm{pH}$ were taken by the same instrument, symPHony SP90M5, and their associated probes which were able to monitor the temperature to normalize the recorded data to the reference temperature of $25{ }^{\circ} \mathrm{C}$. Calibrating the instrument and conductivity probe with the standard $\mathrm{NaCl}$ solution of $20.00 \mathrm{mS} / \mathrm{cm}$ resulted in linear temperature compensation factor $(\alpha)$ of $0.013{ }^{\circ} \mathrm{C}^{-1}$. Cell coefficient was precisely determined to three significant digits before each set of measurements. The calibration of $\mathrm{pH}$ electrode was conducted with buffer solutions and resulted in linear temperature compensation $\alpha$ of $-0.01 \mathrm{pH} /{ }^{\circ} \mathrm{C}$, which was in good agreement with that provided in the datasheet of the buffer solutions.

The conductivity and $\mathrm{pH}$ measurements of the pore solutions extracted after 30 minutes from mixing the materials for two typical pastes are shown in Tables 4.3 and 4.4. The typical change of paste and pore solution resistivity with water to cement ratio were also plotted in Fig. 4.3 and 4.4 for these two paste samples. 
Table 4.3. Pore solution conductivity/resistivity and $\mathrm{pH}$ results for OPC plus $30 \%$ fly ash at $30^{\text {th }}$ minute of hydration age.

\begin{tabular}{|lcccc|}
\hline \multicolumn{1}{|c}{ Paste ID } & $\mathbf{w} / \mathbf{c}$ & $\begin{array}{c}\boldsymbol{\sigma}_{25} \text { pore solution } \\
(\mathrm{mS} / \mathrm{cm})\end{array}$ & $\begin{array}{c}\boldsymbol{\rho}_{25} \text { pore solution } \\
(\Omega . \mathrm{m})\end{array}$ & $\mathbf{p H}$ 25 pore solution \\
\hline P0.35-FA30 & 0.3 & 52.8 & 0.1894 & 13.33 \\
P0.35-FA30 & 0.35 & 50.7 & 0.1972 & 13.32 \\
P0.4-FA30 & 0.4 & 44.1 & 0.2268 & 13.30 \\
P0.45-FA30 & 0.45 & 41.3 & 0.2421 & 13.28 \\
P0.5-FA30 & 0.5 & 38.9 & 0.2571 & 13.26 \\
\hline
\end{tabular}

Table 4.4. Pore solution conductivity/resistivity and $\mathrm{pH}$ results for OPC plus $0.5 \%$ superplasticizer at $30^{\text {th }}$ minute of hydration age.

\begin{tabular}{|lcccc|}
\hline \multicolumn{1}{|c|}{ Paste ID } & $\mathbf{w} / \mathbf{c}$ & $\begin{array}{c}\boldsymbol{\sigma}_{25} \text { pore solution } \\
(\mathrm{mS} / \mathrm{cm})\end{array}$ & $\begin{array}{c}\boldsymbol{\rho}_{25} \text { pore solution } \\
(\Omega . \mathrm{m})\end{array}$ & $\mathbf{p H}$ 25 pore solution \\
\hline P0.3-SP0.5 & 0.3 & 75.4 & 0.1326 & 13.44 \\
P0.35-SP0.5 & 0.35 & 62.8 & 0.1592 & 13.42 \\
P0.4-SP0.5 & 0.4 & 57.2 & 0.1748 & 13.40 \\
P0.45-SP0.5 & 0.45 & 52.8 & 0.1894 & 13.37 \\
\hline
\end{tabular}




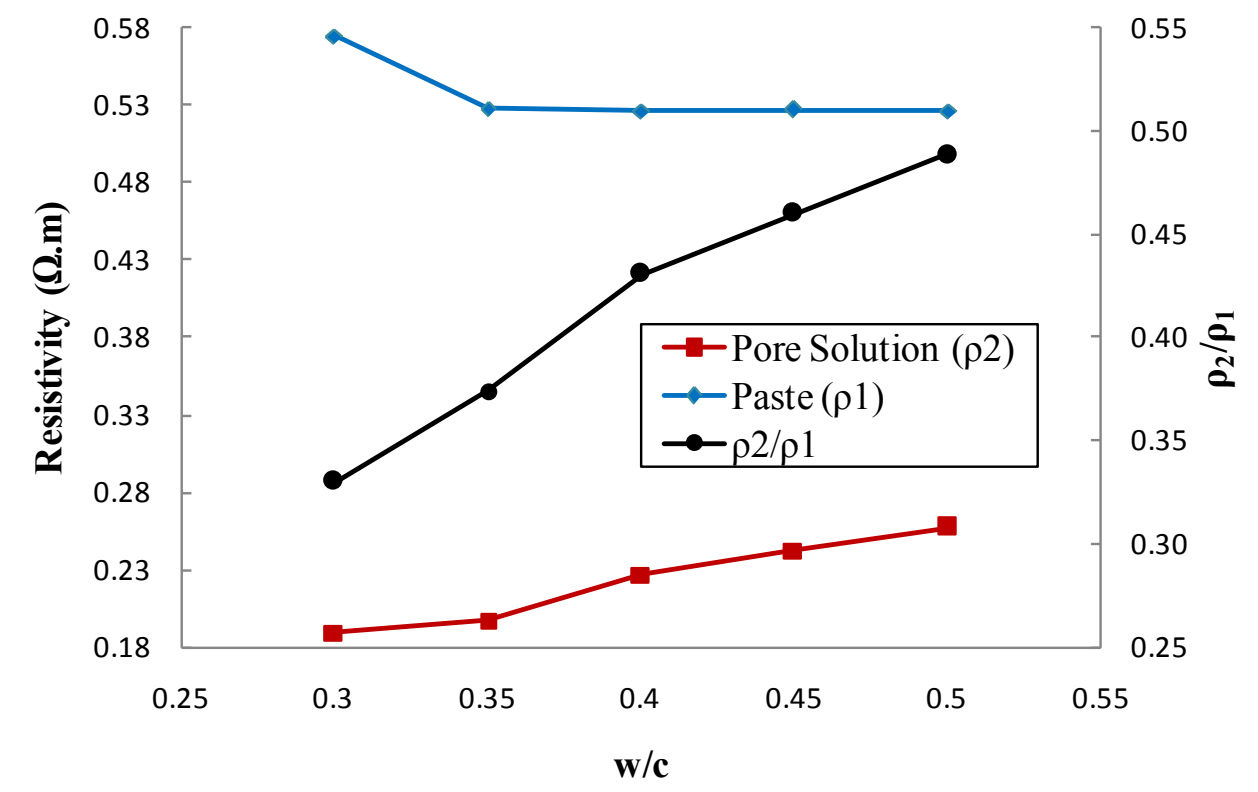

Figure 4.3. Paste and pore solution resistivity variations with $\mathrm{w} / \mathrm{c}$ and their ratio for OPC plus $30 \%$ fly ash at $30^{\text {th }}$ min.

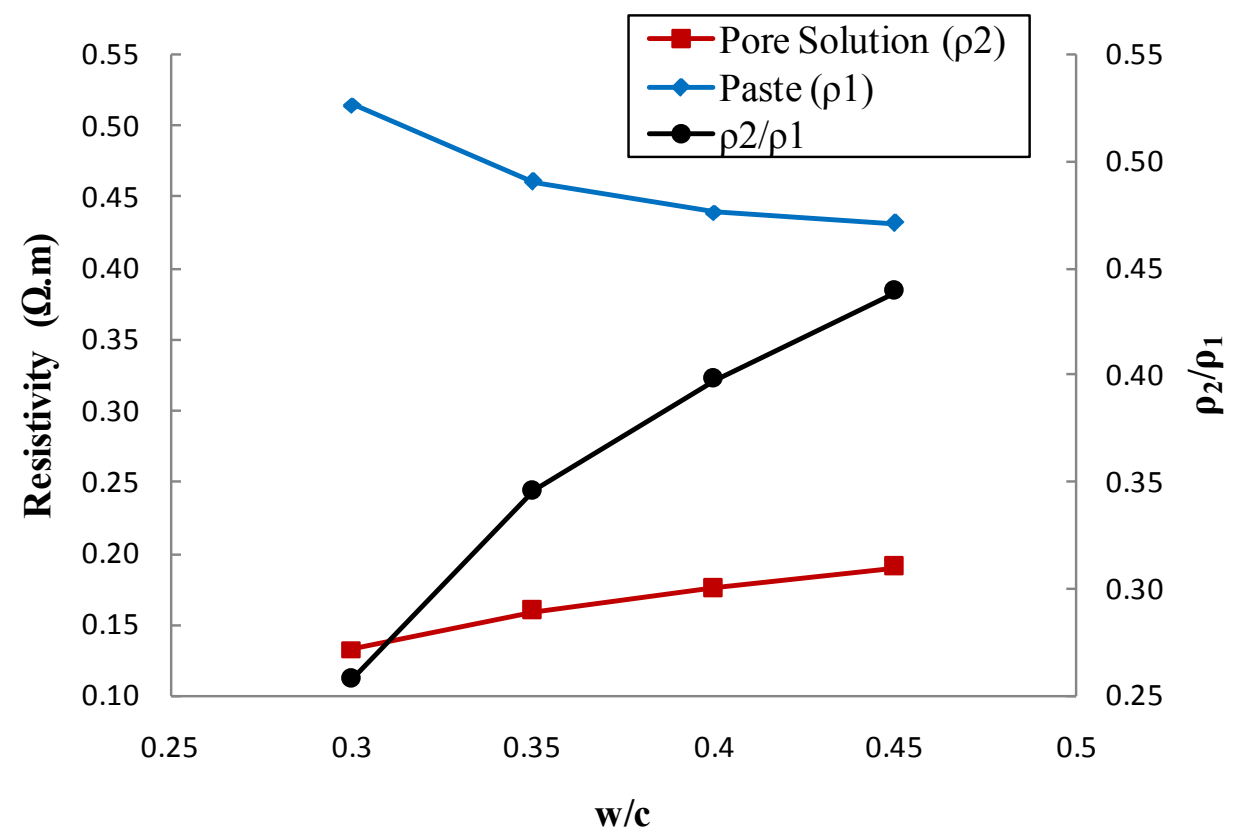

Figure 4.4. Paste and pore solution resistivity variations with $\mathrm{w} / \mathrm{c}$ and their ratio for OPC plus $0.5 \%$ superplasticizer at $30^{\text {th }} \mathrm{min}$. 
The reproducibility of our experimental data was tested for some select pastes with varying water-cement ratios. The average of the test data from reproducibility tests as well as error bars with one standard deviation from average were plotted to make sure that our results were consistent (Fig. 4.5).

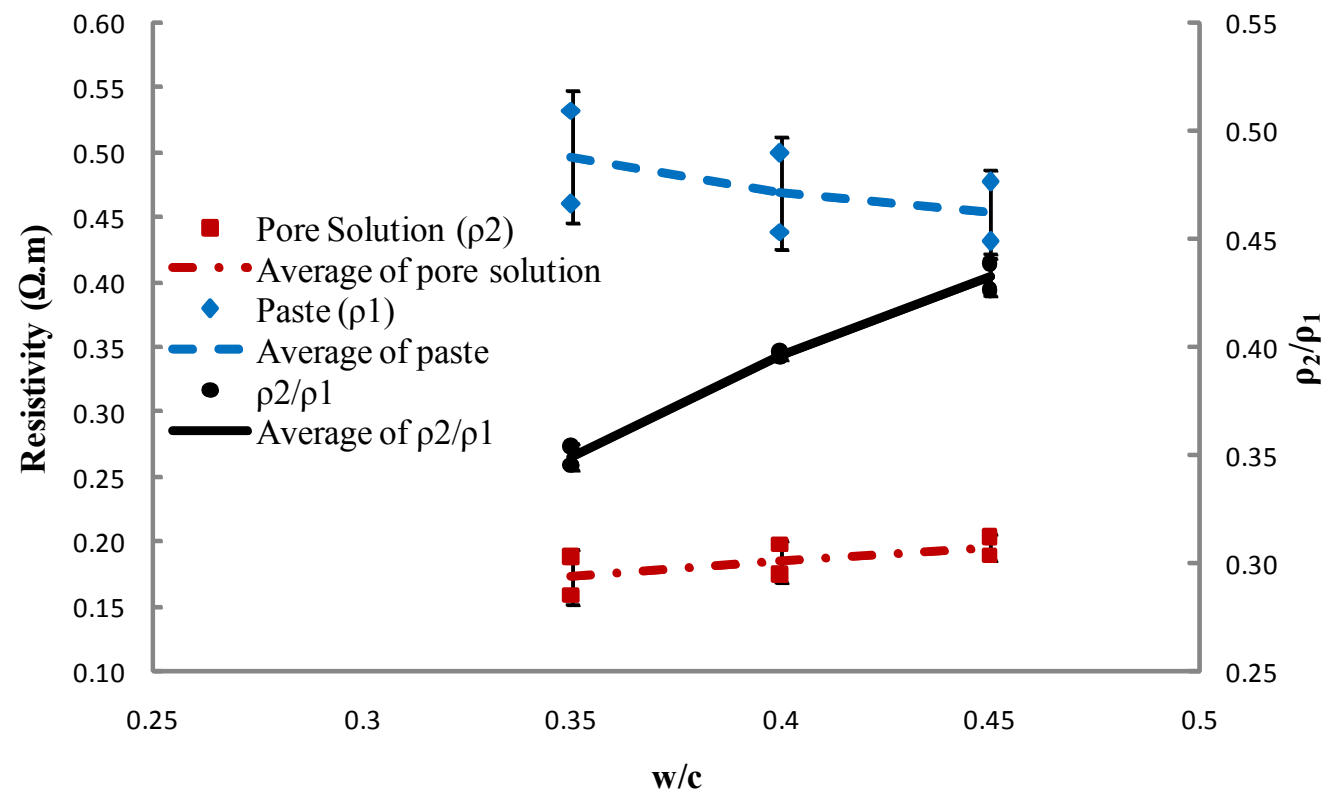

(a)

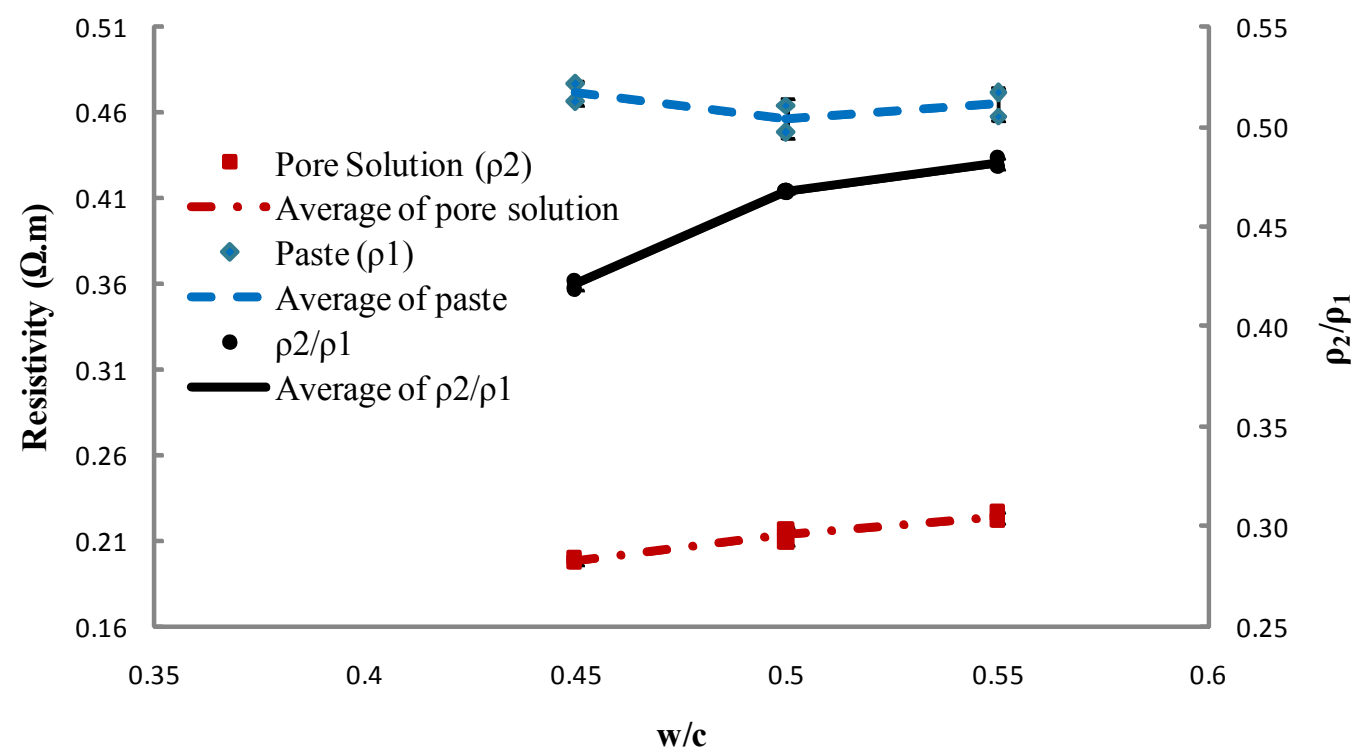

(b) 


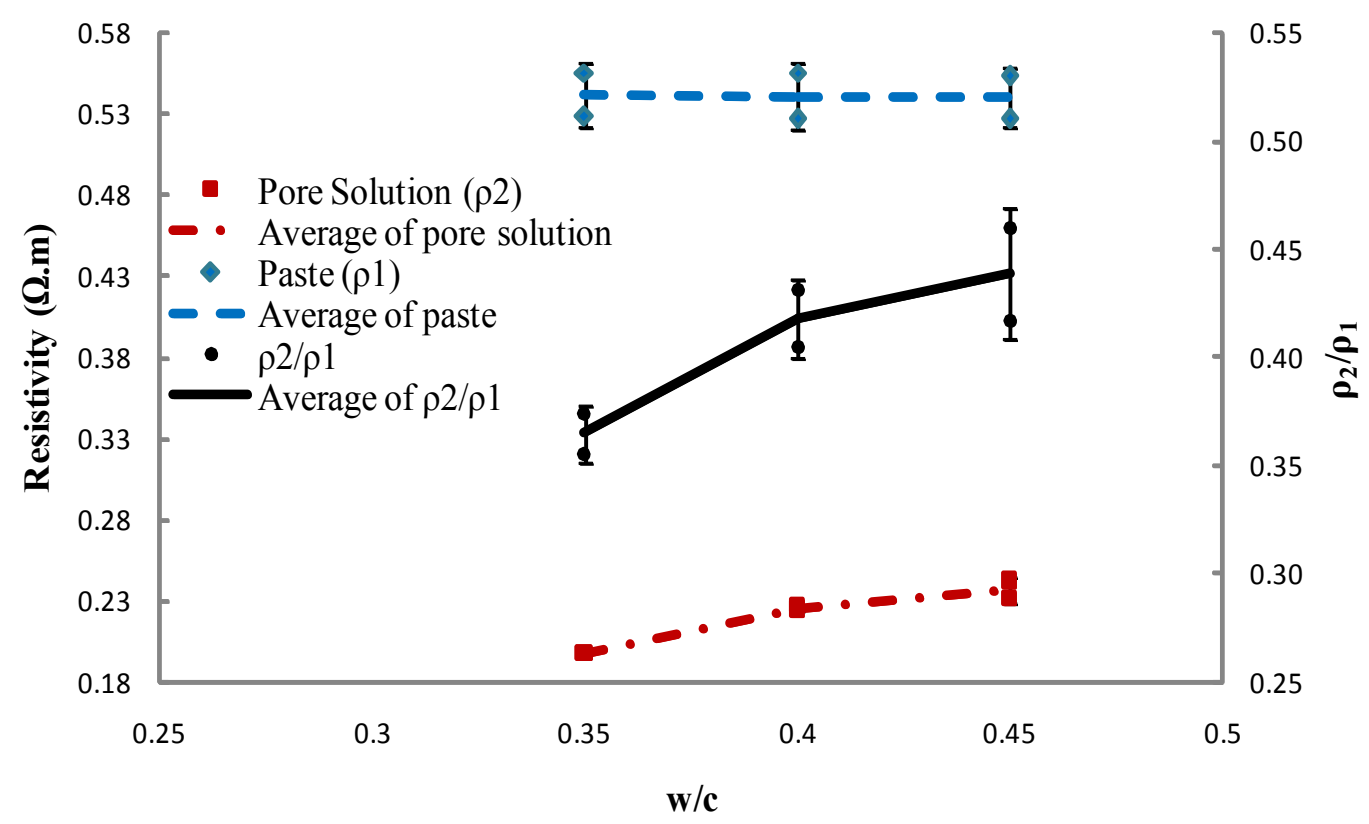

(c)

Figure 4.5. Resistivity results from reproducibility tests and their average as well as error bars with 1 standard deviation from average: a) OPC plus $0.5 \%$ superplasticizer; b) OPC plus $10 \%$ silica fume; c) OPC plus $30 \%$ fly ash.

The plots in Fig. 4.5 reveal that the resistivity of pore solution, dash dot line, increases with increasing the $\mathrm{w} / \mathrm{c}$ of paste which corresponds to decrease in pore solution conductivity. The descending trend in conductivity or ascending in the resistivity of pore solution by increasing the w/c was observed in all of the extracted samples (91 samples including reproducibility tests). The conductivity of pore solution is related to ions transport which depends on the concentration of ions in the pore solution. In other words, the more the w/c, the less the concentration of ions per unit volume of pore solution, which corresponds to a lower conductivity and higher resistivity. Hence, since pore 
solution acts like an electrolytic solution, its conductivity only depends on chemical dissolution reactions resulting in ions release in pore solution.

On the other hand, the resistivity of the paste which is the resistivity of a two-component matrix with pore solution as a liquid phase and cementitious materials as a solid phase, depends on pore solution conductivity (chemical effect) and pores volume in the matrix (physical effect) as well as solid microstructure (physical effect). Increasing the w/c of the paste increases the pore solution resistivity; hence, from the chemical perspective, resistivity of the paste should increase. However, higher amount of liquid in the paste results in lower amount of solid particles in the matrix; therefore, the paste resistivity should decrease from the physical point of view. Accordingly, these two contradicting effects determine whether the resistivity of paste would increase or decrease with increasing the w/c. This depends on which of these two effects is the governing factor. As it can be seen in Fig. 4.5, in most of the cases the physical effect was dominant; i.e., the higher $\mathrm{w} / \mathrm{c}$ resulted in less paste resistivity. But, in some cases for high w/c ratios the chemical effect was governed and increase in pore solution resistivity masked the effect of pores volume (physical effect). This contradictory effect in paste resistivity trend suggested that we considered the resistivity ratio (i.e., the resistivity of the paste to the resistivity of pore solution) to evaluate the effect of w/c ratio. Fig 4.5 shows that by increasing the $\mathrm{w} / \mathrm{c}$ in pastes, ratio of the pore solution resistivity to that of the paste, $\rho_{2} / \rho_{1}$, increases significantly. This ratio and its relationship with other influential parameters will be discussed in details later in Section 4.3.4. In this section only the selected test results representing the typical test data were presented. The supplementary test data are provided in Appendix B. 


\subsection{Discussion}

\subsubsection{Effect of time on conductivity/resistivity development of fresh paste}

To investigate the effect of time after mixing the materials, during the first two hours before initiation of setting, five cement paste mixtures were selected all with the same $\mathrm{w} / \mathrm{c}$ ratio of 0.45 . This $\mathrm{w} / \mathrm{c}$ is very common in construction industry and was in the middle of the w/c range (i.e., 0.3 to 0.55 ) that we used in this study. The time intervals at which the extraction of pore solution was preformed were $30^{\text {th }}, 60^{\text {th }}$ and $90^{\text {th }}$ minutes after mixing the paste. For all of five paste mixtures (i.e., P0.45, P0.45-SP0.5, P0.45-FA30, P0.45-SF10, P0.45-SL30) the conductivity and $\mathrm{pH}$ tests on both paste and its pore solution were conducted and repeated to make sure that the data obtained from experiments were reliable and reproducible.

The results of resistivity/conductivity measurements versus time are presented in Table 4.5 and associated graphs were plotted in Fig. 4.6. Error bars with one standard deviation from average of replicate tests at each measurement time are shown in Fig. 4.6 in order to consider the error of the measured data in the analysis and discussions. 
Table 4.5. Average resistivity of fresh paste and pore solution at $30^{\text {th }}, 60^{\text {th }}$ and $90^{\text {th }}$ minute after mixing for paste mixtures with w/c of 0.45 .

\begin{tabular}{|lccc|}
\hline \multirow{2}{*}{ Paste ID } & $\begin{array}{c}\text { Time } \\
(\mathrm{min})\end{array}$ & $\begin{array}{c}\boldsymbol{\rho}_{25} \text { pore solution } \\
(\Omega . \mathrm{m})\end{array}$ & $\begin{array}{c}\boldsymbol{\rho}_{25} \text { paste } \\
(\Omega . \mathrm{m})\end{array}$ \\
\hline \multirow{3}{*}{ P0.45 } & 30 & 0.1895 & 0.4355 \\
& 60 & 0.1826 & 0.4228 \\
& 90 & 0.1786 & 0.4114 \\
\hline \multirow{3}{*}{ P0.45-SP0.5 } & 30 & 0.1946 & 0.4581 \\
& 60 & 0.1897 & 0.4534 \\
P0.45-SF10 & 90 & 0.1863 & 0.4439 \\
& 30 & 0.1984 & 0.4714 \\
& 60 & 0.1887 & 0.4572 \\
P0.45-FA30 & 90 & 0.1792 & 0.4471 \\
& 30 & 0.2376 & 0.5369 \\
& 60 & 0.2286 & 0.5260 \\
P0.45-SL30 & 90 & 0.2213 & 0.5149 \\
\hline \multirow{3}{*}{} & 30 & 0.2651 & 0.5886 \\
& 60 & 0.2592 & 0.5764 \\
& 90 & 0.2480 & 0.5620 \\
\hline
\end{tabular}

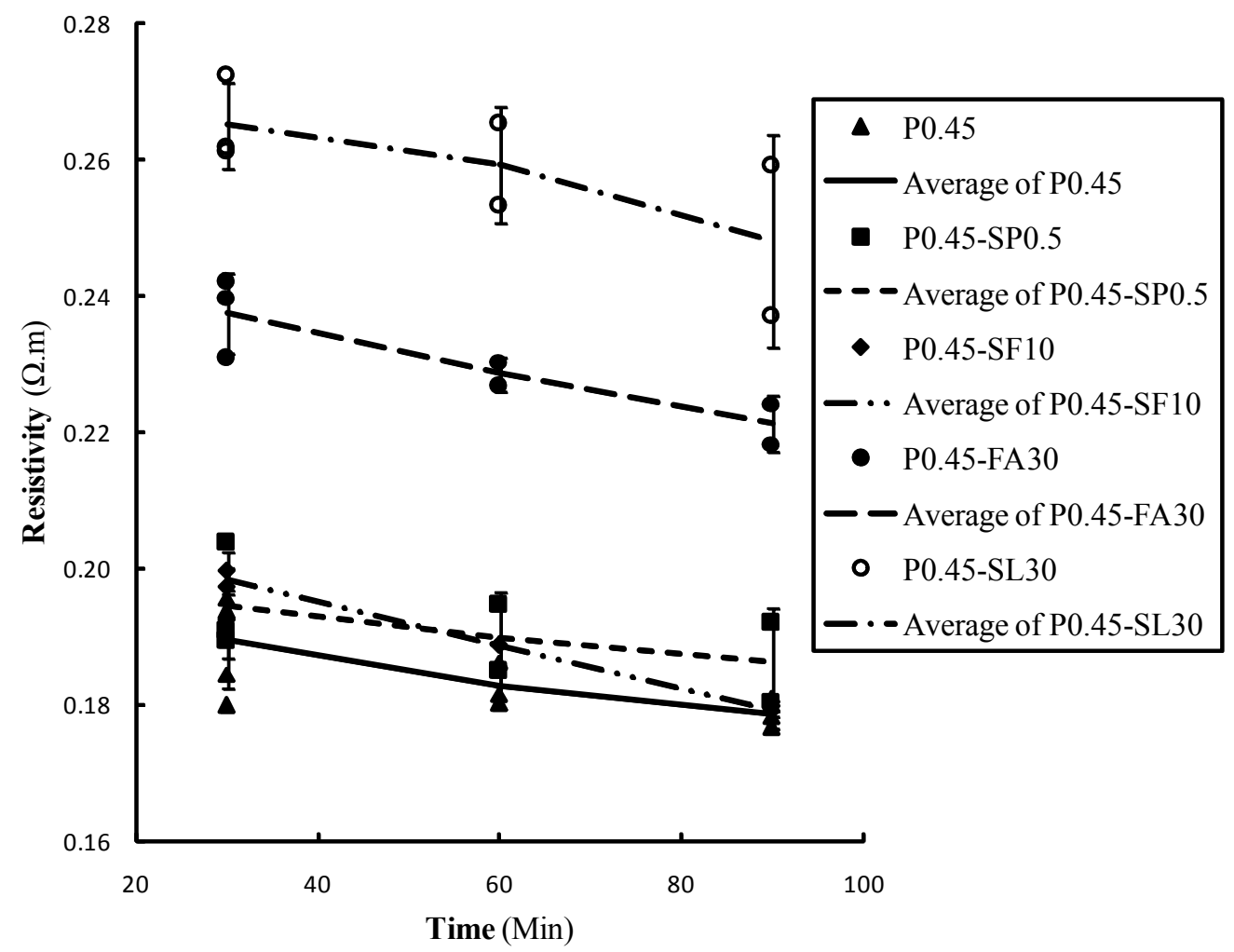

(a) 


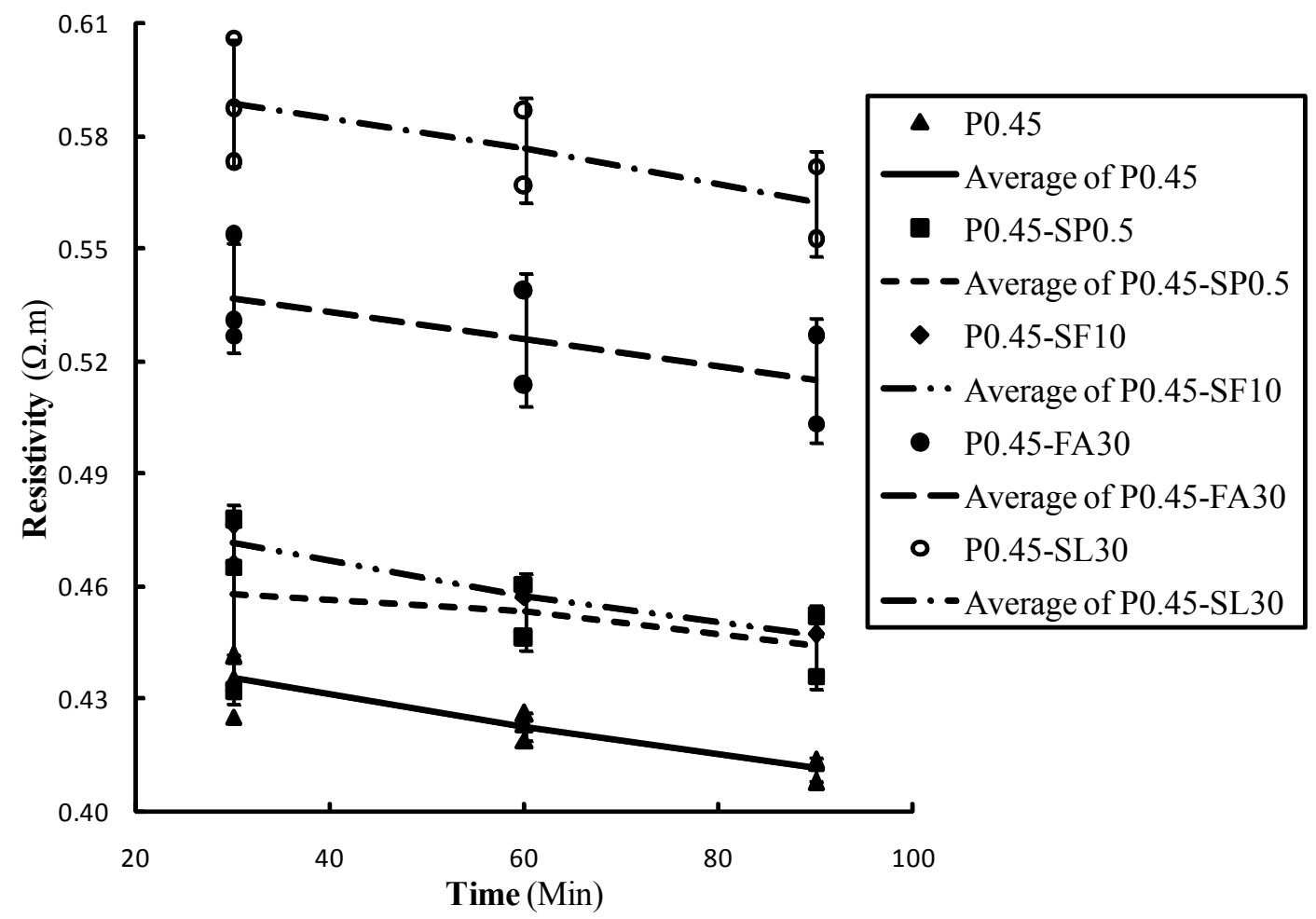

(b)

Figure 4.6. Electrical resistivity development with time during fresh state and error bars with one standard deviation from average for the paste mixtures with w/c of $0.45:$ a) pore solution; b) paste.

The graphs in Fig. 4.6 exhibit a constant descending trend in the pore solution resistivity which was valid for all the paste mixtures. The decrease in resistivity corresponds to an increase in the electrical conductivity. During the first 2 hours after mixing, the electrical conductivity of the liquid component (i.e., pore solution) increases which is attributed to the chemical reactions between cementitious materials and the water present in the mixture. As distilled water, with its close-to-zero conductivity, is added to the cementitious materials, the oxides and alkalies in the cementitious materials react with water molecules. As a result, ions are released in the pore solution and the concentration 
of ions increases. Therefore, the electrical conductivity of the pore solution increases over time. The increase of the electrical conductivity in the pore solution follows an exponential function in which during the first few minutes right after mixing, it shows a sharp increase, while the rate of growth gradually decreases [14] due to the saturation of the pore solution with alkali and hydroxide ions

On the other hand, the paste resistivity also decreased during this stage before setting as shown in Fig. 4.6. The decrease in resistivity during this period indicated that the ratio of volume of pore solution to that of solid particles is almost constant within two hours after mixing the cement paste before the time of setting. Whereas, during and after the setting time, because of the formation of hydration products the ratio (porosity) is not constant and decreases $[11,15]$. Therefore, the concentration of released ions in the pore solution has a dominant effect in reducing the resistivity of pastes in the fresh state.

The pore solution conductivity gradually increases even after setting time and for the hardening state which corresponds to a constant descending order in pore solution resistivity. The results of conductivity of pore solution squeezed out from selected hardened paste samples at around 5 months age, are shown in Fig. 4.7. It reveals that pore solution conductivity for all types of paste mixtures significantly increases at hardened stage compared to that of fresh stage. Concentration of ions released into pore solution depends on quantity of them (moles) released as a result of reaction between cementitious material particles and water, the portion which is taken up by hydration products (i.e., CRPs as discussed in section 4.2), and the pore solution volume which decreases after setting because of bound water in hydration products structures [37]. Therefore, the 
increase in ions concentration at hardened state indicates that ions taken up which reduces ions concentration is not governing effect, while the decrease in pore solution volume as well as increase in the amount of ions because of hydration progress are dominant factors in increasing the corresponding ions concentration. However, Fig. 4.7 shows that in samples with $10 \%$ of silica fume, the pore solution conductivity does not increase significantly. This can be most likely attributed to much higher potential of taking up the ions by $\mathrm{C}-\mathrm{S}-\mathrm{H}$ in samples with silica fume in which the ratio of $\mathrm{Ca} / \mathrm{Si}$ drops significantly because of quite high percentage of $\mathrm{SiO}_{2}$ in chemical composition of silica fume particles [37]. Thus, lower ratio of $\mathrm{Ca} / \mathrm{Si}$ in $\mathrm{C}-\mathrm{S}-\mathrm{H}$ products of silica fume incorporated pastes, increases ability to take up the released ions which results in lower increase in pore solution conductivity compared to other types of paste mixtures. In contrast, the paste resistivity initially drops to a minimum value during the fresh state and gradually increases during setting period and significantly in hardening state [14]. This is related to the solidification process resulting from the formation of the hydration products as well as the reduction of pore solution volume in the paste system. 


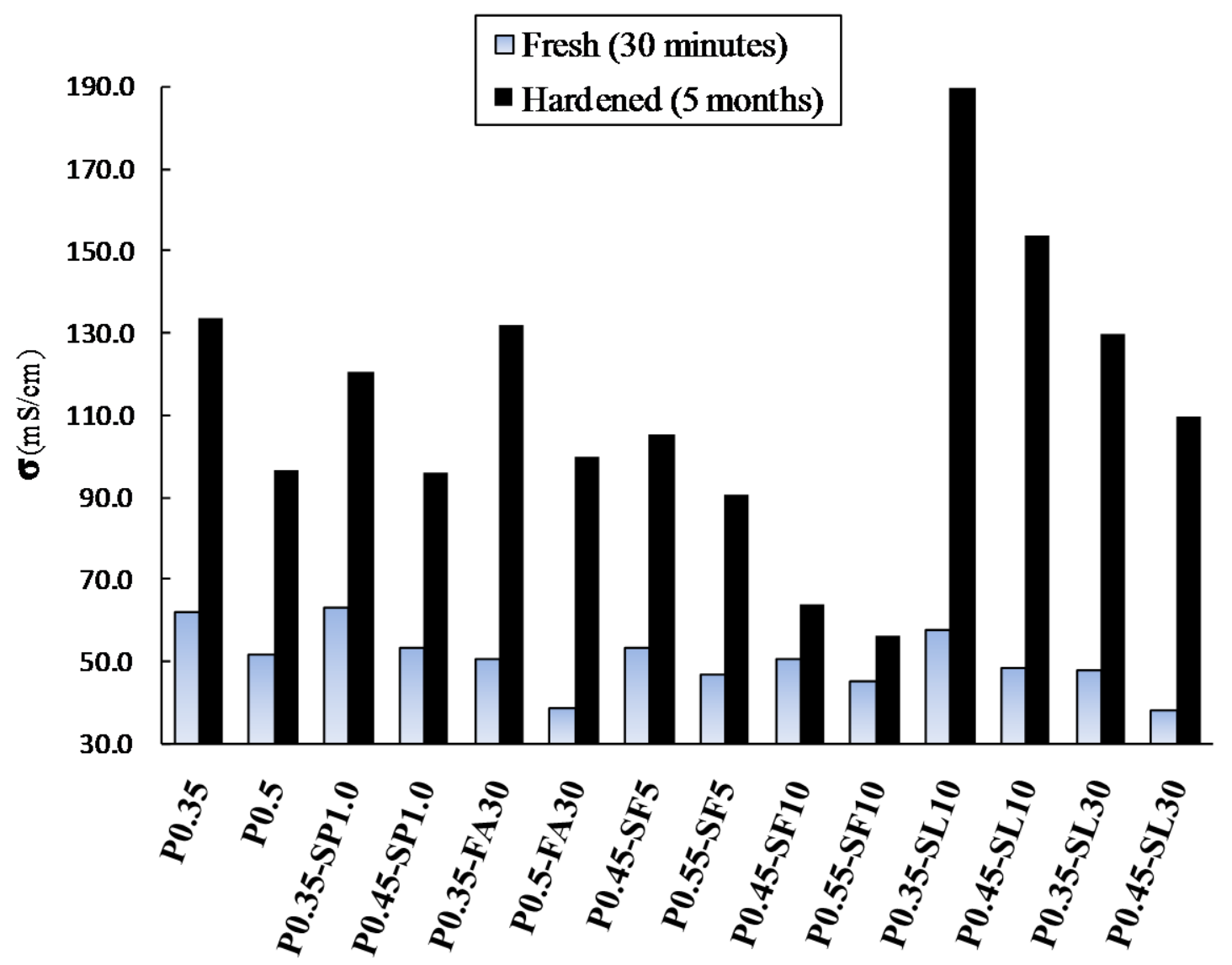

Fig. 4.7. Pore solution conductivity of selected paste mixtures at fresh state compared to that of hardened state at around 5 months old.

\subsubsection{Effect of superplasticizer and SCMs on pore solution conductivity}

To study the effect of chemical admixtures and SCMs on the conductivity of fresh paste system, the most commonly used SCMs (such as silica fume, slag and flay ash) as well as a polycarboxylate-based superplasticizer were used. The SCMs affect the characteristics of the solid phase in the cement paste system and the superplasticizer changes the characteristics of both the solid phase and the liquid phase compared to the control paste with only the ordinary portland cement and distilled water. The size of the SCMs 
particles and their distribution in the water are different from those of the OPC. The superplasticizer changes the distribution of the cement particles and also the conductivity of the pore solution. In this research various paste mixtures with a constant w/c of 0.45 was used to study the effect of these auxiliary materials on the resistivity of fresh paste system. All the measurements were conducted at $30^{\text {th }}, 60^{\text {th }}$ and $90^{\text {th }}$ minute after mixing the materials. Besides, medium dosages of additives/admixtures that are commonly used in concrete industry were used as follows: $\mathrm{SP}=0.5 \mathrm{wt} \%$ of cementitious materials, $\mathrm{FA}=30 \mathrm{wt} \%$ of cementitious materials, $\mathrm{SF}=10 \mathrm{wt} \%$ of cementitious materials and $\mathrm{SL}=30 \%$ of cementitious materials. The tests were conducted on three repeating mixtures and the average of results was calculated and reported. The results of pore solution conductivity tests were presented in Table 4.6 and the graphs were plotted in Fig. 4.8.

Table 4.6. Pore solution conductivity of 5 main types of paste mixtures for $30^{\text {th }}, 60^{\text {th }}$ and $90^{\text {th }}$ minute of hydration age at $\mathrm{w} / \mathrm{c} 0.45$.

\begin{tabular}{|c|c|c|c|c|c|}
\hline \multirow{2}{*}{ Time } & \multicolumn{5}{|c|}{$\sigma_{25}$ Pore Solution $(\mathrm{mS} / \mathrm{cm})$} \\
\cline { 2 - 6 }$(\mathrm{min})$ & P0.45 & P0.45-SP0.5 & P0.45-FA30 & P0.45-SF10 & P0.45-SL30 \\
\hline 30 & 52.78 & 51.38 & 42.08 & 50.40 & 37.72 \\
60 & 54.76 & 52.72 & 43.75 & 53.00 & 38.58 \\
90 & 55.99 & 53.68 & 45.19 & 55.80 & 40.32 \\
\hline
\end{tabular}




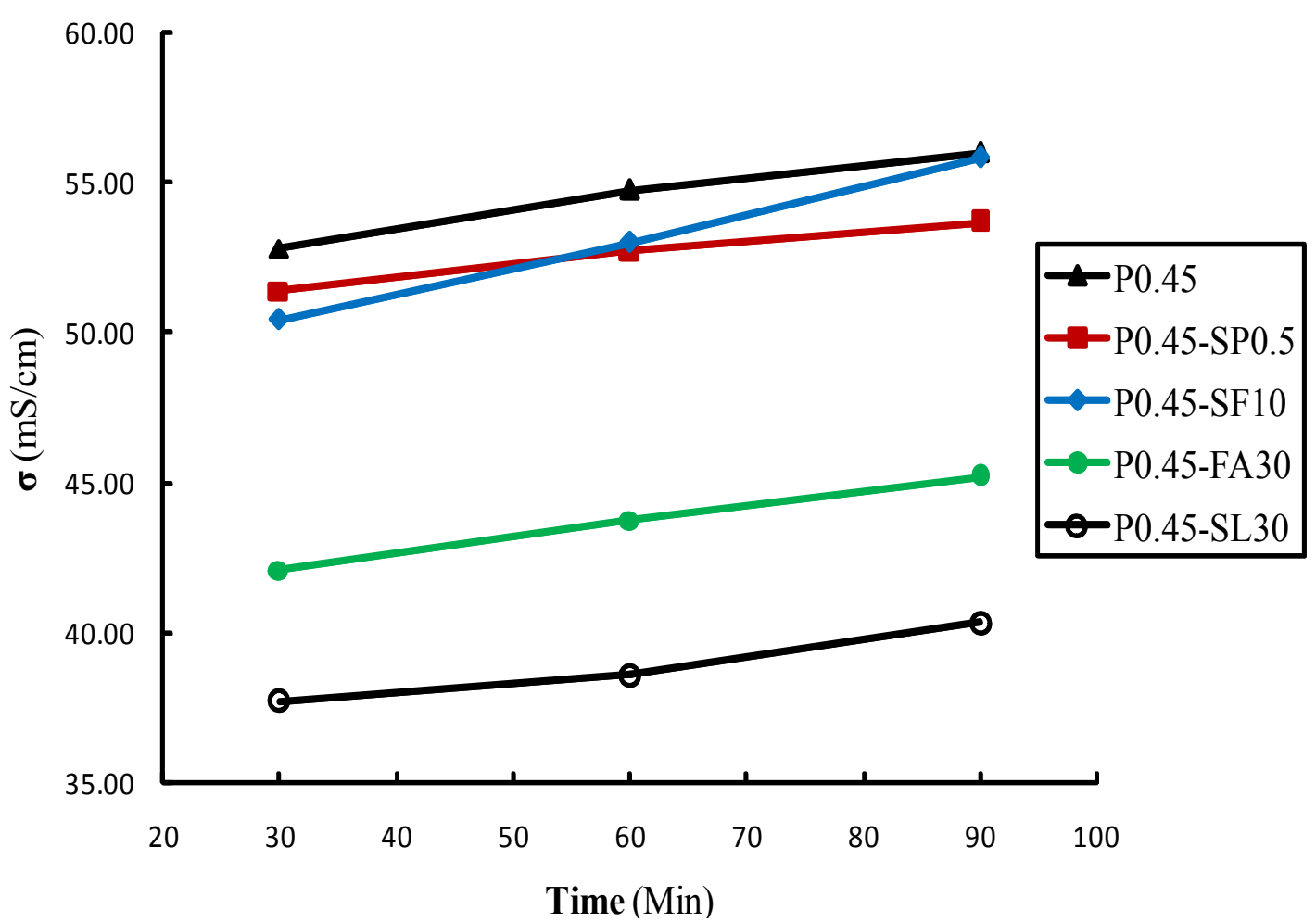

Figure 4.8. Pore solution conductivity ( $\sigma$ pore solution $)$ development with time for five general types of paste mixtures including OPC, OPC plus $0.5 \%$ superplasticizer, OPC plus $30 \%$ fly ash, OPC plus $10 \%$ silica fume and OPC plus $30 \%$ slag at w/c 0.45 .

Fig. 4.8 shows that at the fresh state, the control mixture (OPC) has the highest pore solution conductivity compared to that of pastes containing SCMs; i.e., adding the SCMs such as fly ash, silica fume or slag reduces the electrical conductivity of pore solution. This can be attributed to the concentration of released contributing ions such as $\mathrm{OH}^{-}, \mathrm{K}^{+}$ and $\mathrm{Na}^{+}$to the electrical conductivity of the pore solution[13, 18]. When the SCM is added to the control mixture, the concentration of the released ions decreases because, before the setting, the only parameter that affects the pore solution conductivity is the concentration of ions in the pore solution. 
The superplasticizer used in the experiments was a poly-carboxylate based admixture which increases the flowability and workability of paste and reduces the water required in mixture proportion as well as delaying the setting to give construction staff more time for pumping and compaction. The graph in Fig. 4.8 exhibits a small drop (around 4\%) in pore solution conductivity values when the superplasticizer is used in the mixture. The amount of ions released from the chemical reactions between cementitious materials and water, are almost constant compared to the control mixture because of the same chemical composition of cementitious materials in solid component. Therefore, the concentration of ions in the pore solution does not change in the presence of super plasticizer. However, the dynamic viscosity $(\mu)$ of pore solution increases because of higher viscosity of superplasticizer compared to that of the distilled water. Increase in pore solution viscosity reduces ions transport [60]. Therefore, the equivalent conductivity of ions species decreases. In other words, having the same ionic concentration in a more viscous pore solution containing superplasticizer results in less pore solution conductivity.

The silica fume incorporated pastes also have less pore solution conductivity than that of the OPC mixtures at earlier ages due to the lower concentration of released ions in the pore solution which delayed hydration reactions $[9,13]$. However, as shown in Fig. 4.8, the rate of increase in pore solution conductivity due to the progress of chemical reactions (i.e., ion dissolutions) from silica fume particles in water is more than the OPC paste. This is most likely related to the fineness $\left(\mathrm{cm}^{2} / \mathrm{g}\right)$ of silica fume particles; the fineness of the silica fume particles is significantly higher compared to that of other cementitious materials such as OPC, fly ash and slag. The silica fume fineness or surface area is around $200,000 \mathrm{~cm}^{2} / \mathrm{g}$, while the finesse of the OPC, fly ash and slag is about 4,000 
$\mathrm{cm}^{2} / \mathrm{g}$. The surface area of silica fume particles that are in contact with water is higher and therefore, the rate of ion dissolutions after mixing is higher compared to the OPC paste.

Pastes with fly ash or slag have more significant drop in pore solution conductivity compared to OPC paste as shown in Fig. 4.8. The conductivity drop in slag incorporated pastes is the highest among all the paste mixtures. Because, the delayed reactions of these pozzolans with water results in less released ions in pore solution to that of OPC particles $[10,13]$. Additionally, the lower conductivity of slag blended pastes compared to fly ash incorporated ones is most likely attributed to more delayed hydration in its particles as well as less alkalis $\left(\mathrm{K}_{2} \mathrm{O}\right.$ and $\left.\mathrm{Na}_{2} \mathrm{O}\right)$ contents in its chemical composition, which leads to less amount of ions released in pore solution. Thus, the concentration of alkali cations $\mathrm{K}^{+}$ and $\mathrm{Na}^{+}$in pore solution decreases in both pastes containing fly ash and slag compared to that of OPC which is more significant in latter; i.e., huge drop in pore solution conductivity of slag incorporated pastes occurs.

The slopes of lines in Fig. 4.8 also show that the rates of conductivity increase with time in all types of paste mixtures are in the same order except silica fume blended paste. The slope of the line for the silica fume paste is around twice of others. The rate of ion release during the hydration of solid particles is higher in silica fume which is most likely attributed to a major difference in its surface area which is around 50 times higher than that of other cementitious materials such as OPC, fly ash and slag. This quite high surface area results in faster rate of reactions with water. 


\subsubsection{Effect of $\mathrm{w} / \mathrm{c}$ on pore solution conductivity}

Water to cementitious materials ratio, $\mathrm{w} / \mathrm{c}$, in the paste mixtures is determined as the ratio between the mass of liquid phase/s to that of solid phase/s (i.e., cementitious binders). To observe and investigate the effect of w/c on the pore solution conductivity/resistivity of a particular type of paste, a certain hydration age was selected in order to eliminate the time effect in our experiments. Considering the conductivity increase with time as discussed in Section $4.3 .1,30^{\text {th }}$ minute after mixing the materials was chosen as the most appropriate time for data comparison and analysis. The selected time during fresh state (i.e., around 2 hours) should be closer to that of mixing of materials to make sure that setting has not initiated; however, during the very first few minutes the conductivity gain is too sharp and it is not practical to do the measurement accurately. Besides, the most common time of concrete pouring in ready mix concrete industry is around half an hour. Therefore, the age of 30-minutes after mixing was selected as the time of pore solution extraction and conductivity measurement. The results obtained at $30^{\text {th }}$ minute for various paste mixtures are shown in Fig. 4.9.

The range of $w / c$ for each paste mixture was appropriately selected to achieve an adequate homogeneity and cohesiveness and avoid segregation in fresh paste. These factors limited the maximum and minimum w/c value used in this study. For instance, since silica fume considerably decreases the flowability of the paste whereas the superplasticizer improves its flowability. Therefore, the range of w/c for silica fume pastes was from 0.4 to 0.55 while the w/c ratio for superplasticizer incorporated pastes ranged from 0.3 to 0.45 . 


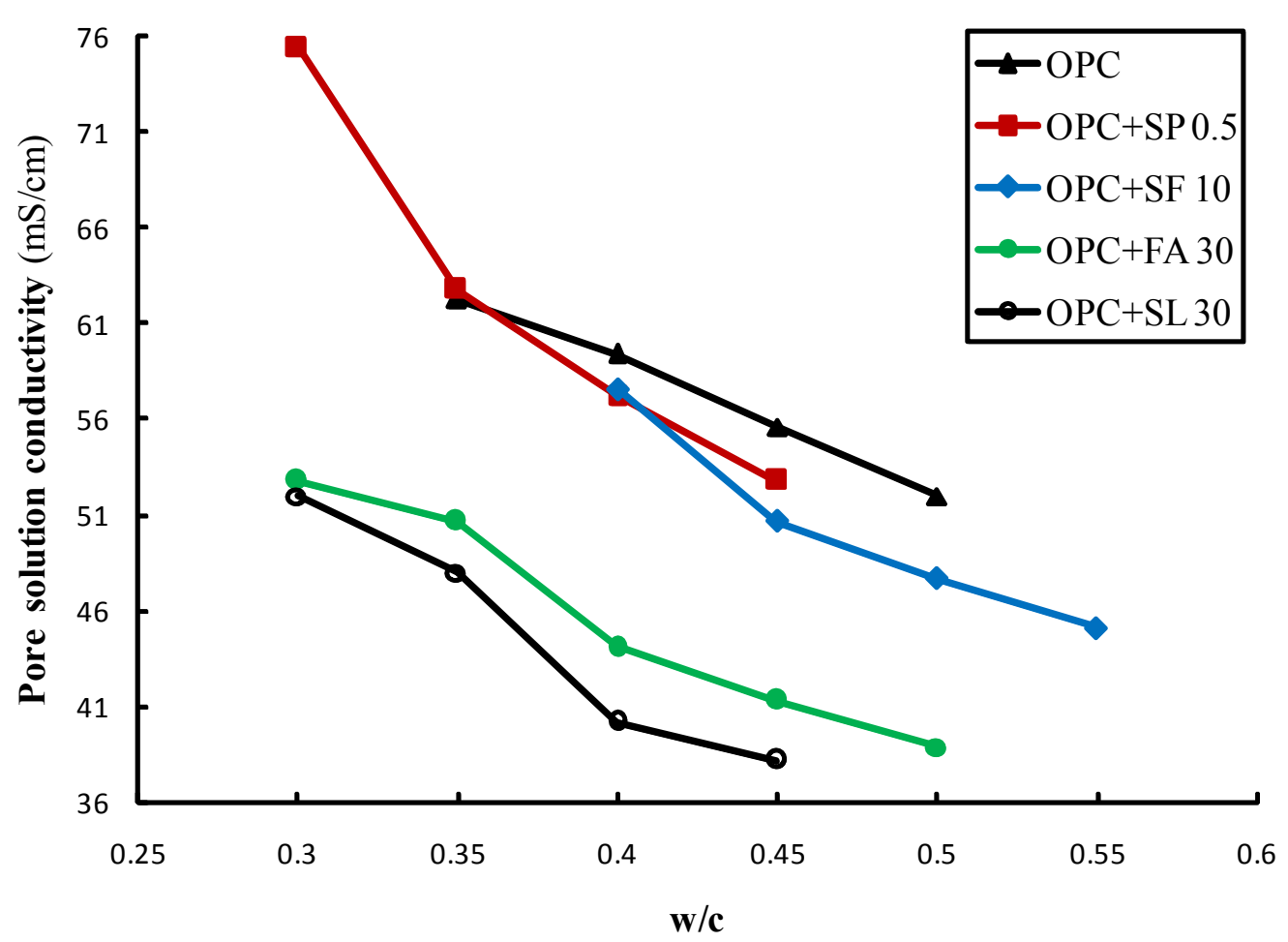

Figure 4.9. Pore solution conductivity versus $\mathrm{w} / \mathrm{c}$ ratio for paste samples at $30^{\text {th }}$ minute of hydration age.

The graphs in Fig. 4.9 confirm descending trend for pore solution conductivity with increase in $w / c$ for all paste mixtures; i.e. the higher the $\mathrm{w} / \mathrm{c}$ of a paste is, the lower the corresponding pore solution electrical conductivity becomes. The reduced conductivity as a result of increasing the liquid content of the paste is attributed to the lower concentration of dissolved ions in the pore solution.

The concentration of significant ions (i.e., $\mathrm{K}^{+}$and $\mathrm{Na}^{+}$) in the pore solution is defined as the quantity of ions released into pore solution per unit volume $\left(\mathrm{cm}^{3}\right)$ of the pore solution. For a certain type of paste mixture such as OPC or OPC plus silica fume, the liquid content increases at higher $\mathrm{w} / \mathrm{c}$ ratios. As a result, at any hydration age (e.g., $30^{\text {th }}$ minute), 
the pore solution volume ncreases, while the amount of released $\mathrm{K}^{+}$and $\mathrm{Na}^{+}$ions into pore solution does not change since it is a function of reacted fraction of cementitious material as well as its total alkali contents $\left(\mathrm{K}_{2} \mathrm{O}\right.$ and $\left.\mathrm{Na}_{2} \mathrm{O}\right)$ which are both independent from w/c. Therefore, since by definition ionic concentration is inversely proportional to pore solution volume, when pore solution volume decreases with increasing w/c ratio, this corresponds to reduction in pore solution conductivity.

\subsubsection{Relationship between the electrical resistivity of paste and pore solution using Archie's law}

The Archie's law was presented in 1942 for 100\% saturated sandstones [19] and later further developed by Atkins and Smith [57] in the form of Eq. 2.27. Using the same approach for the fresh cement paste after mixing in which the saturation degree is close to $100 \%$ and no water is bounded as well as no solid hydration product such as $\mathrm{C}-\mathrm{S}-\mathrm{H}$ is formed, the volume of liquid conductive phase equals that of pores in the paste media and therefore, Eq. 2.27 can be expressed in the following form:

$F=\frac{\rho_{1}}{\rho_{2}}=A \varphi^{-m}$

where $\rho_{1}$ and $\rho_{2}$ are the paste and pore solution resistivity, respectively; $A$ is the constant tortuosity of the paste, $\mathrm{m}$ is the shape factor and $\varphi$ is porosity defined as the fractional volume of the liquid conductive phase (w) in the paste matrix. Through this equation the electrical resistivity of fresh paste is related to the physical arrangement of highly resistive solid particles and the conductivity of the pore solution $[16,58]$. 
Table 4.7. Particle density of the materials used in the paste mixtures at $25^{\circ} \mathrm{C}$.

\begin{tabular}{|c|c|c|c|c|c|}
\hline \multicolumn{6}{|c|}{ Particle Density $\left(\mathbf{g} / \mathbf{c m}^{3}\right)$} \\
\hline Water & Superplasticizer & OPC & Fly ash & Silica fume & Slag \\
\hline 1.00 & 1.00 & 3.14 & 2.70 & 2.30 & 2.90 \\
\hline
\end{tabular}

Having the weighted water to cementitious materials ratio, w/c, and the density of the solid and liquid substances, D, in Table 4.7 as well as the replacement ratio of supplementary cementitious materials, the porosity $\varphi$ is calculated as follows:

$\varphi=\frac{V_{w}}{V_{w}+V_{c}}=\frac{V_{w}}{V_{w}+V_{O P C}+V_{S C M}}$

$\varphi=\frac{\frac{w}{D_{w}}}{\frac{w}{D_{w}}+\frac{m_{O P C}}{D_{O P C}}+\frac{m_{S C M}}{D_{S C M}}}$

where $\mathrm{m}_{\mathrm{OPC}}$ and $\mathrm{m}_{\mathrm{SCM}}$ are the weight (mass) of OPC and supplementary cementitious materials, respectively; and $\mathrm{D}_{\mathrm{w}}$ is the liquid content density. Substituting the value of liquid content density from Table 4.7 (i.e., $1.00 \mathrm{~g} / \mathrm{cm}^{3}$ ) into Eq. 4.3, it can be rewritten as follows:

$\varphi=\frac{w}{w+\frac{m_{O P C}}{D_{O P C}}+\frac{m_{S C M}}{D_{S C M}}}$ 
Given the total weight of the cementitious materials, $\mathrm{c}$, and dividing the numerator and denominator by c and substituting into the Eq. 4.4 results in:

$$
\varphi=\frac{w / c}{w / c+\frac{(1-R)}{D_{O P C}}+\frac{R}{D_{S C M}}}
$$

where $\mathrm{D}_{\mathrm{OPC}}$ and $\mathrm{D}_{\mathrm{SCM}}$ are the particle density of the ordinary portland cement and supplementary cementitious material, respectively; and R is the SCM replacement ratio to the total cementitious materials; e.g. $\mathrm{R}$ is 0.1 for $10 \%$ silica fume incorporated paste mixture.

Eq. 4.5 exhibits that porosity and w/c are directly proportional when $\mathrm{D}_{\mathrm{SCM}}$ and $\mathrm{R}$ are constants; i.e., the greater the designated w/c, the higher the paste porosity for a particular paste mixture. The following two sections describe the results of studying variation of the formation factor $(\mathrm{F})$ in pastes with w/c and porosity.

\subsubsection{Linear approach $(\mathrm{m}=1)$}

The linear approach to the Archie's law (Eq. 4.1) regards the particular condition in which exponent $\mathrm{m}$ is equal to 1. Accordingly, Eq. 4.1 can be simplified to Eq. 4.6 such that

$$
\frac{1}{F}=\frac{1}{A} \times \varphi
$$

Defining inverse of Tortuosity as pore connectivity $[11,61]$, then:

$$
\frac{1}{F}=\frac{\rho_{2}}{\rho_{1}}=\beta \varphi
$$


where $\rho_{1}$ and $\rho_{2}$ are the paste and pore solution resistivity, respectively; $\varphi$ is the porosity of paste; and $\beta$ is the pore connectivity. Connectivity factor $\beta$ represents how close the paste behaves to parallel model resistors and it ranges from 0 to 1 ; i.e., the greater the $\beta$, the closer the paste behavior to that of parallel resistors (solid phase and pore solution) in an electrical circuit.

To investigate the effect of $w / c$ on the paste resistivity and its relationship with that of pore solution, resistivity measurements were conducted on different paste mixtures 30 minutes after mixing. The typical results for paste mixtures with mid-dosage of SCMs or superplasticizer are shown in Fig. 4.10 and other results for low and high dosages can be found in Fig. B.1 in Appendix B.

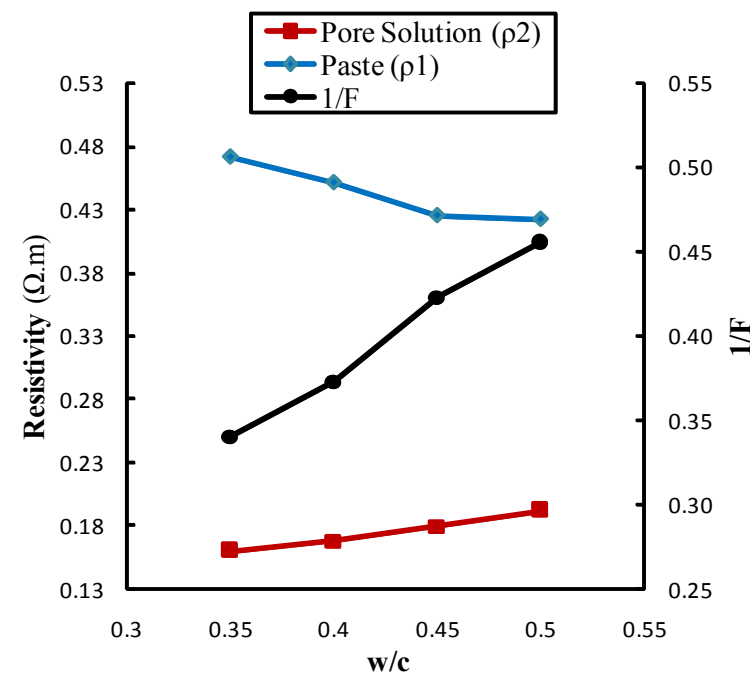

(a)

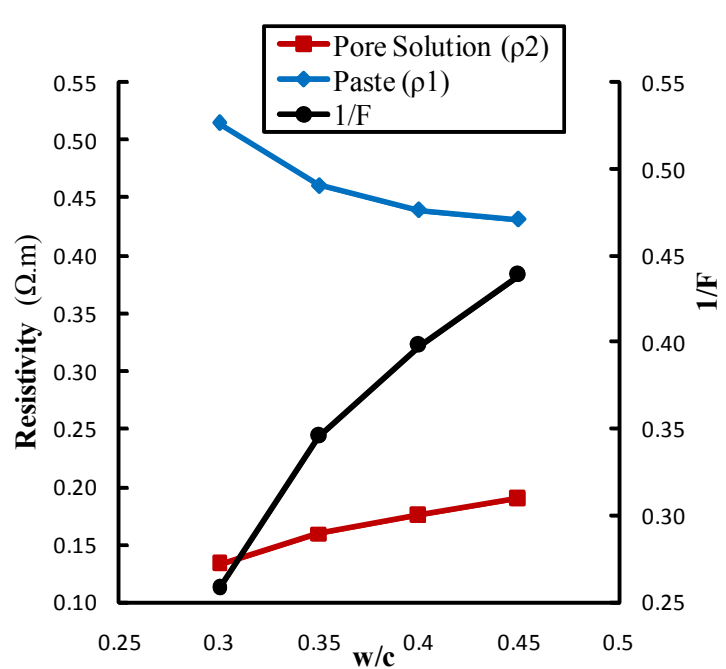

(b)

Figure 4.10. Paste and pore solution resistivity as well as inverse of formation factor versus w/c at $30^{\text {th }}$ minute of paste age: a) Ordinary Portland Cement; b) OPC plus $0.5 \%$ superplasticizer; c) OPC plus 30\% fly ash; d) OPC plus $10 \%$ silica fume; and e) OPC plus $30 \%$ slag. 


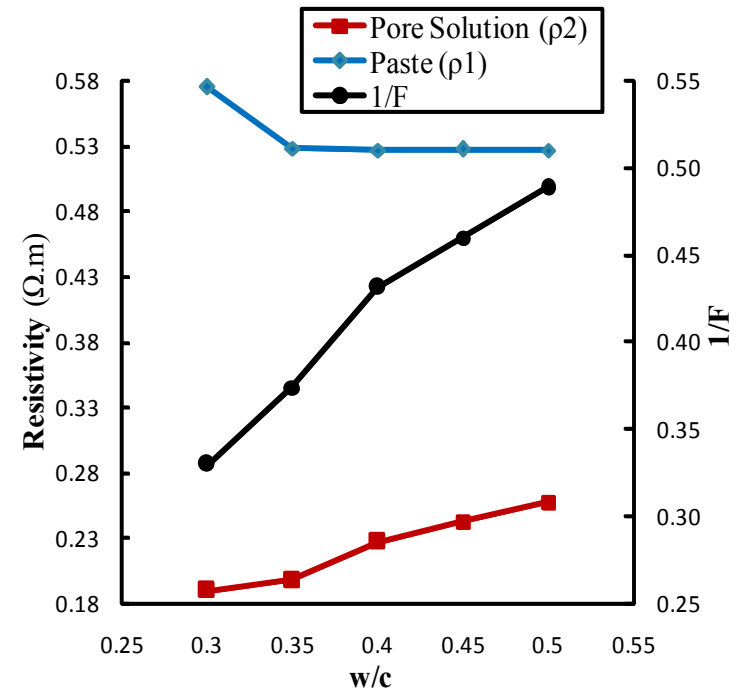

(c)

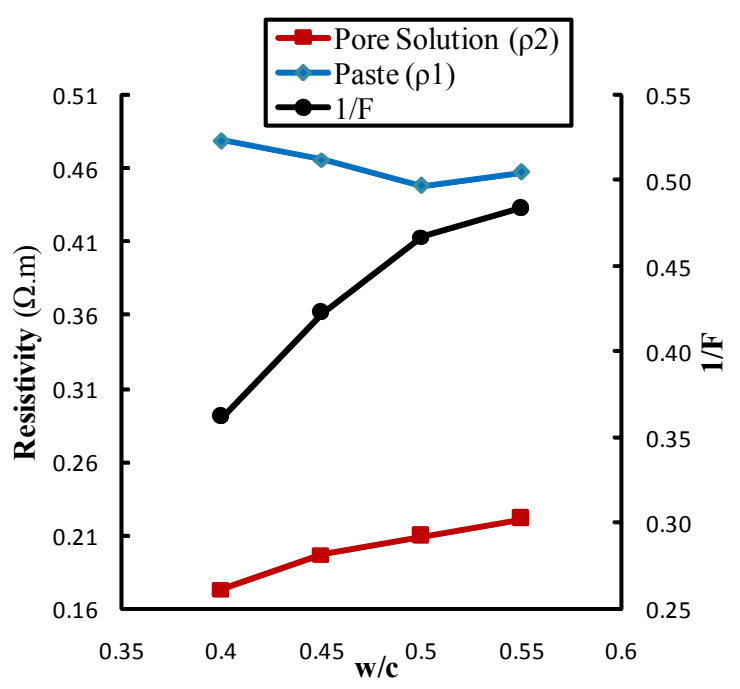

(d)

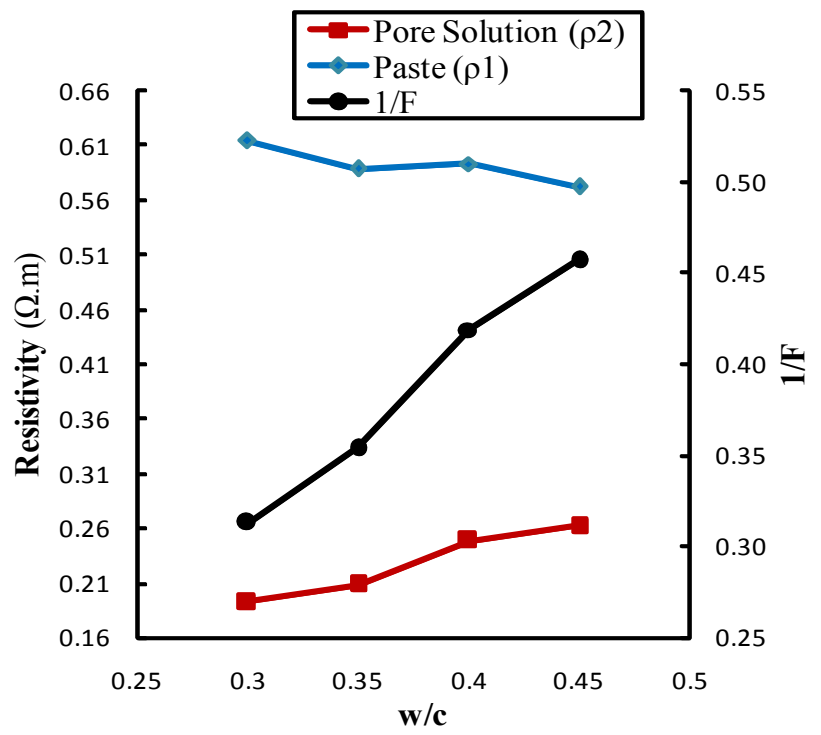

(e)

Figure 4.10 (continued). Paste and pore solution resistivity as well as inverse of formation factor versus w/c at 30th minute of paste age: a) Ordinary Portland Cement; b) OPC plus $0.5 \%$ superplasticizer; c) OPC plus $30 \%$ fly ash; d) OPC plus $10 \%$ silica fume; and e) OPC plus 30\% slag. 
Fig. 4.10 shows that if the liquid content of paste and thus its porosity increases, paste resistivity exhibits a small drop for the low and medium w/cs ( 0.3 to 0.4$)$, while this drop reduces or becomes negligible for higher w/cs ( 0.4 to 0.55$)$. This is attributed to the opposing factors affecting the paste resistivity with the change in w/c. From a chemical point of view, increase in w/c or porosity results in lower conductivity which corresponds to higher pore solution resistivity (as discussed in Section 4.3.3). However, from physical perspective, in higher w/c pastes, the portion of resistive phase (solid particles) decreases and so the connectivity; accordingly decrease in the paste matrix resistivity is anticipated. In other words, whenever the chemical effect is dominant (e.g. in a very high $w / c$ in silica fume incorporated paste) the resultant paste resistivity increases; otherwise, the physical effect (i.e., $\varphi \beta$ ) governs the decrease in the paste resistivity. This conclusion is quite different from that of Mancio et al. [8] in fresh concrete in which they reported that the electrical resistivity of fresh concrete always increases with increasing the w/c. However, in their concrete mixture proportions, they did not consider the volumetric fraction of the aggregates which was most likely the governing factor in increasing the electrical resistivity at higher w/cs. As shown in Fig. 4.10, in contrary to the paste resistivity, the inverse of formation factor $(1 / F)$, shows a promising ascending trend with w/c.

The connectivity value ( $\beta$ ) calculated from Eq. 4.7 is presented in Table 4.8 for the OPC pastes. Similar to the OPC pastes, the connectivity value of all paste mixtures increased with the increase of w/c. Therefore, the ratio of pore solution resistivity to that of the paste (1/F) was only affected by the physical effect and arrangement of solid particles in the pore solution; i.e. higher w/c resulted in higher porosity as well as higher connectivity and therefore, their product $\varphi \beta$ (i.e., 1/F) increased. 


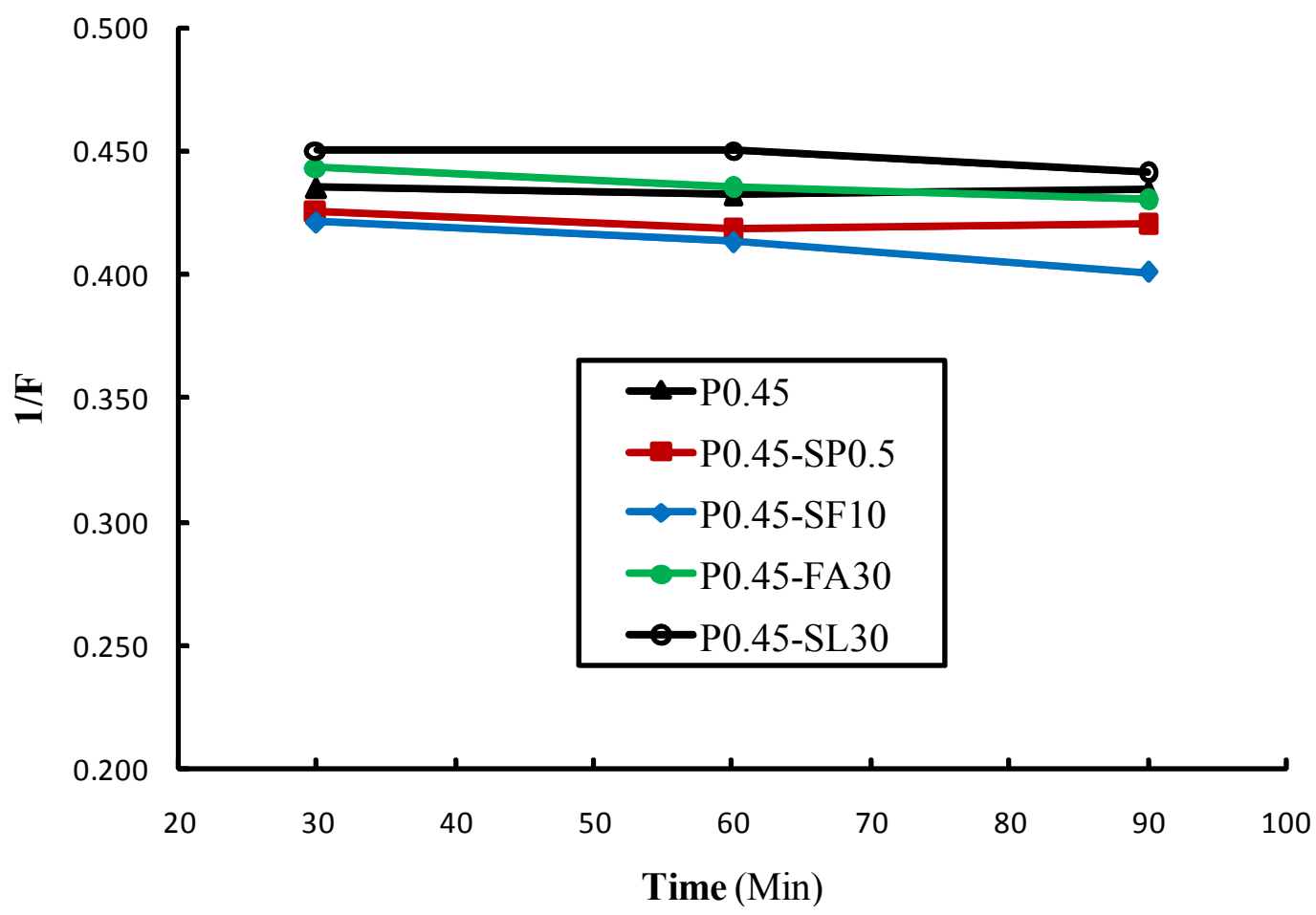

Figure 4.11. Inverse of formation factor, $1 / \mathrm{F}$, versus time for pastes with the w/c of 0.45 during the first 2 hours.

Table 4.8. Variation of porosity $\varphi$ and connectivity $\beta$ with $w / c$ for ordinary portland paste (OPC) at $30^{\text {th }}$ minute of paste age.

\begin{tabular}{|cccc|}
\hline $\mathbf{w} / \mathbf{c}$ & $\boldsymbol{\varphi}$ & $\mathbf{1 / F}$ & $\boldsymbol{\beta}$ \\
\hline 0.35 & 0.5236 & 0.3205 & 0.6121 \\
0.4 & 0.5567 & 0.3731 & 0.6702 \\
0.45 & 0.5856 & 0.4230 & 0.7223 \\
0.5 & 0.6109 & 0.4554 & 0.7454 \\
\hline
\end{tabular}


Table 4.9. Inverse of formation factor, $1 / \mathrm{F}$, versus time for pastes with the $\mathrm{w} / \mathrm{c}$ of 0.45 during the first 2 hours.

\begin{tabular}{|c|c|c|c|c|c|}
\hline \multirow{2}{*}{ Time } & \multicolumn{5}{|c|}{$\mathbf{1 / F = \varphi \boldsymbol { \beta }}$} \\
\cline { 2 - 6 }$(\mathrm{min})$ & $\mathbf{P 0 . 4 5}$ & $\mathbf{P 0 . 4 5 - S P 0 . 5}$ & $\mathbf{P 0 . 4 5 - F A 3 0}$ & $\mathbf{P 0 . 4 5 - S F 1 0}$ & P0.45-SL30 \\
\hline 30 & 0.435 & 0.425 & 0.443 & 0.421 & 0.450 \\
60 & 0.432 & 0.418 & 0.435 & 0.413 & 0.450 \\
90 & 0.434 & 0.420 & 0.430 & 0.401 & 0.441 \\
\hline
\end{tabular}

The time effect on the paste and pore solution resistivity at the w/c of 0.45 was discussed in Section 4.3.1. It was found that both the paste and pore solution resistivity decrease with time during fresh state which was in agreement with reported data by Sant et al. in the literature for the w/c of 0.3 [11]. However, they did not study the formation factor variation during the first 2 hours and their focus was on the resistivity variation during the setting and hardening stage up until 48 hours. Therefore, further investigation was required to explore the fresh state variation of formation factor.

The inverse of formation factor $(1 / F)$ was investigated in our study during first 2 hours for the w/c of 0.45 . Tthe average results are presented in Table 4.9 and corresponding graphs are shown in Fig. 4.11. Although it was reported $[11,61]$ that after initial setting the porosity $(\varphi)$ and connectivity $(\beta)$ decrease significantly due to hydration products formation, Fig. 4.11 exhibits that during fresh state 1/F is plateau which indicates that $\varphi$ and $\beta$ are almost constant for the first 2 hour period and most likely till setting initiates. Hence, up until initial setting, the solid microstructure of paste (i.e., $\varphi$ or $\beta$ ) does not change and chemical effect determines the paste resistivity. Therefore, resistivity of a 
paste with certain w/c (e.g., 0.45), only depends on corresponding pore solution resistivity and because of decrease in pore solution resistivity with time due to higher ions concentration, paste resistivity also decreases (Fig. 4.6).

\subsubsection{2. $\quad$ Power approach}

The power approach considers the higher rate of decrease in formation factor $\mathrm{F}$ with respect to porosity $(\varphi)$ for lower porosity values and it decreases gradually as the porosity of paste (i.e., the liquid content portion) increases. In other words, the concavity of F- $\varphi$ graph would be upward which is consistent with the results of the experimental data. The typical results for pastes with low and high dosages of SCMs or superplasticizer can be found in Fig. B.2 in Appendix B. In these figures, the formation factor of all paste mixtures decreased with increase in the porosity $\varphi$ of the paste.

The graphs and trend lines in Fig. 4.12 show that regardless of the paste mixture, whether it is incorporated with SCMs such as fly ash, silica fume and slag or chemical admixture such as superplasticizer, Archie's law can be applied to correlate the paste resistivity $\left(\rho_{1}\right)$ to that of the pore solution $\left(\rho_{2}\right)$ through Eq. 4.8. The $R^{2}$ value for all the paste mixtures was higher than 0.92 which indicated a promising correlation between the formation factor $(F)$ and porosity $(\varphi)$ :

$\rho_{1}=\rho_{2} A \varphi^{-m}$

where A is the tortuosity constant of paste obtained experimentally. 


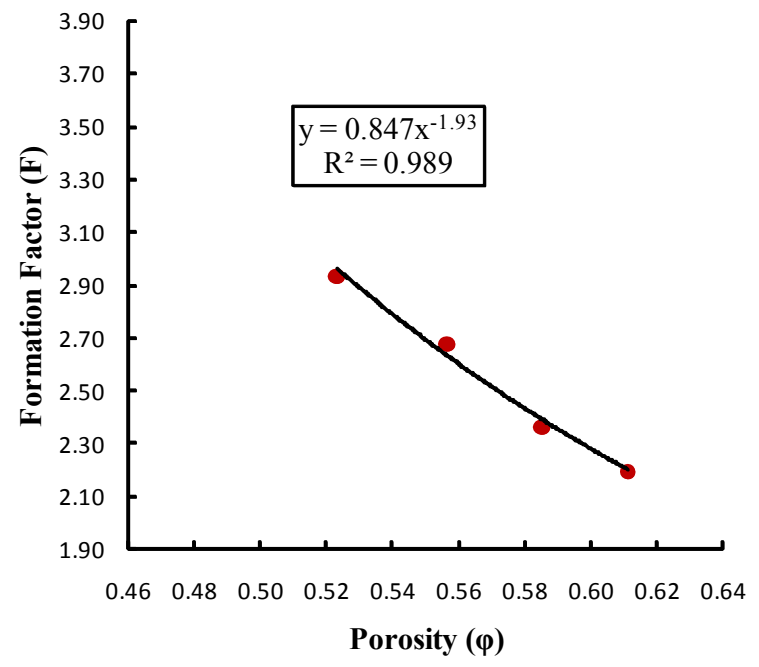

(a)

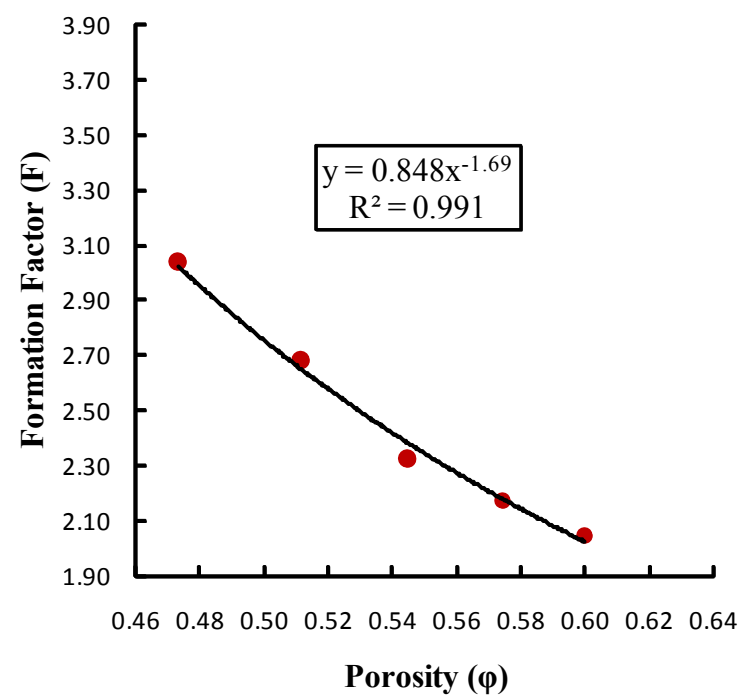

(c)

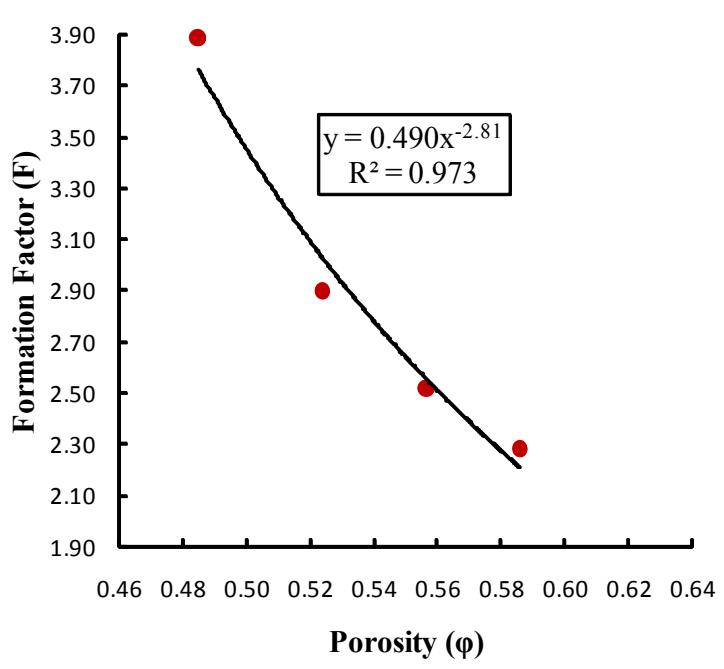

(b)

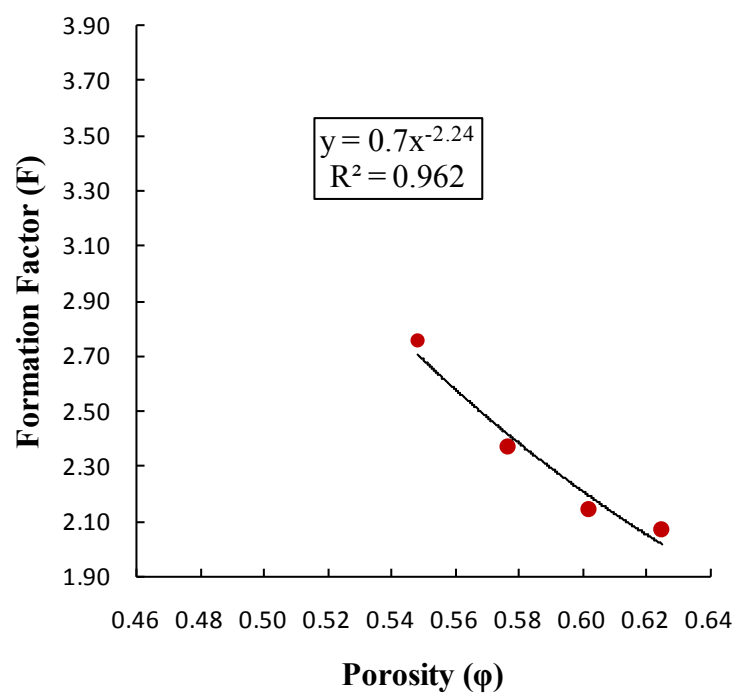

(d)

Figure 4.12. Formation factor versus porosity of fresh cement pastes at $30^{\text {th }}$ minute of paste age: a) Ordinary Portland Cement (OPC); b) OPC plus 0.5\% superplasticizer; c) OPC plus 30\% fly ash; d) OPC plus 10\% silica fume; and e) OPC plus 30\% slag. 


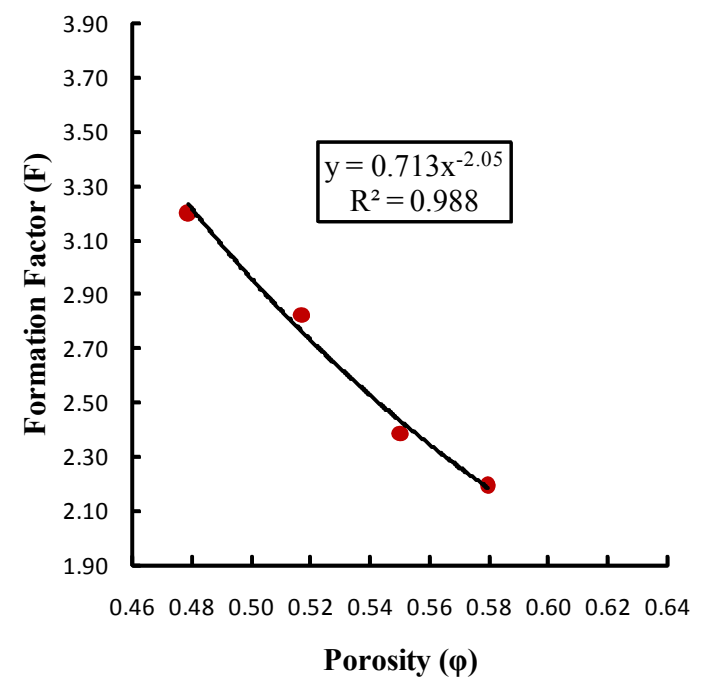

(e)

Figure 4.12 (Continued). Formation factor versus porosity of fresh cement pastes at $30^{\text {th }}$ minute of paste age: a) Ordinary Portland Cement (OPC); b) OPC plus $0.5 \%$ superplasticizer; c) OPC plus 30\% fly ash; d) OPC plus 10\% silica fume; and e) OPC plus $30 \%$ slag.

Eq. 4.8 clearly illustrates the opposing effects of the rise in pore solution resistivity (directly proportional) and porosity (inversely proportional) resulted from increase in the w/c of the paste on the paste resistivity; i.e., for a particular mixture proportion of paste in which tortuosity A is constant, if $\mathrm{w} / \mathrm{c}$ of paste increases, the paste resistivity decreases when physical effect (effect of $\varphi$ ) governs, whereas it increases when chemical effect (effect of $\rho_{2}$ ) is dominant. The latter mostly occurs in high w/c ratios.

From the established fitted curve for the OPC mixture, the values of 0.85 and 1.93 were obtained for the parameters A and $\mathrm{m}$ in Archie's law, respectively. They were close to Li. et al's values; i.e., 0.68 and 1.8 for A and m, respectively [5]. It is noted that the pore 
solution extraction in that study was extracted at the time of minimum critical point, while our measurements were all conducted at $30^{\text {th }}$ minute of hydration age which resulted in more reliable data. Furthermore, the $\mathrm{m}$ range in our results were in good agreement with the predicted values (1.3-2.2) by Pirson for cemented rocks [56].

We also tried to fit one curve to all different paste mixtures to see how accurate the correlation still is for a simplified unified equation in terms of $\mathrm{R}^{2}$ value. Fig. 4.13 shows that the $\mathrm{R}^{2}$ value for all the samples would be 0.85 which is relatively high. If only one simplified equation is desirable in practice, the tortuosity A and exponent m of Archie's law should be taken as 0.79 and 1.99 , respectively.

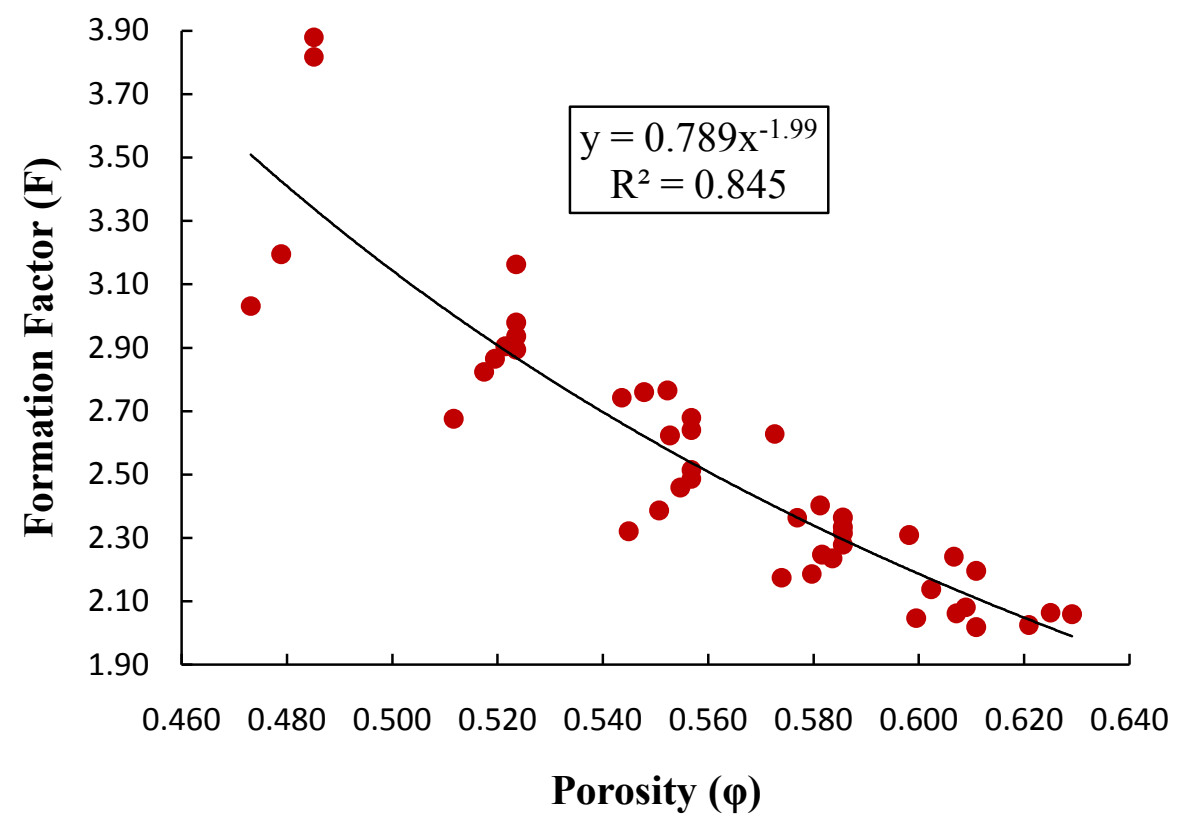

Figure 4.13. Formation factor versus porosity of fresh cement paste at $30^{\text {th }}$ minute of paste age considering all different paste mixtures. 


\subsubsection{Tortuosity:}

Tortuosity is a parameter quantifying the extent of geometric complexity of a porous media. It characterizes the extent of convoluted pathways of electrical conduction (ions transport) through a porous media such as cement paste. In this study a reference value of 1.99 for parameter $\mathrm{m}$ was selected in order to calculate and compare the tortuosity of various paste mixtures. By replacing $\varphi^{-1.99}$ with $\varphi^{\prime}$ in Eq. 4.1, the tortuosity can be calculated from the linear regression analysis as follows:

$F=A \varphi^{-1.99}=A \varphi^{\prime}$

The regression analysis conducted on the OPC paste mixture data is shown in Fig. 4.14 as a typical example. Using this method the tortuosity values of all the paste mixtures were obtained and demonstrated in Fig. 4.15.

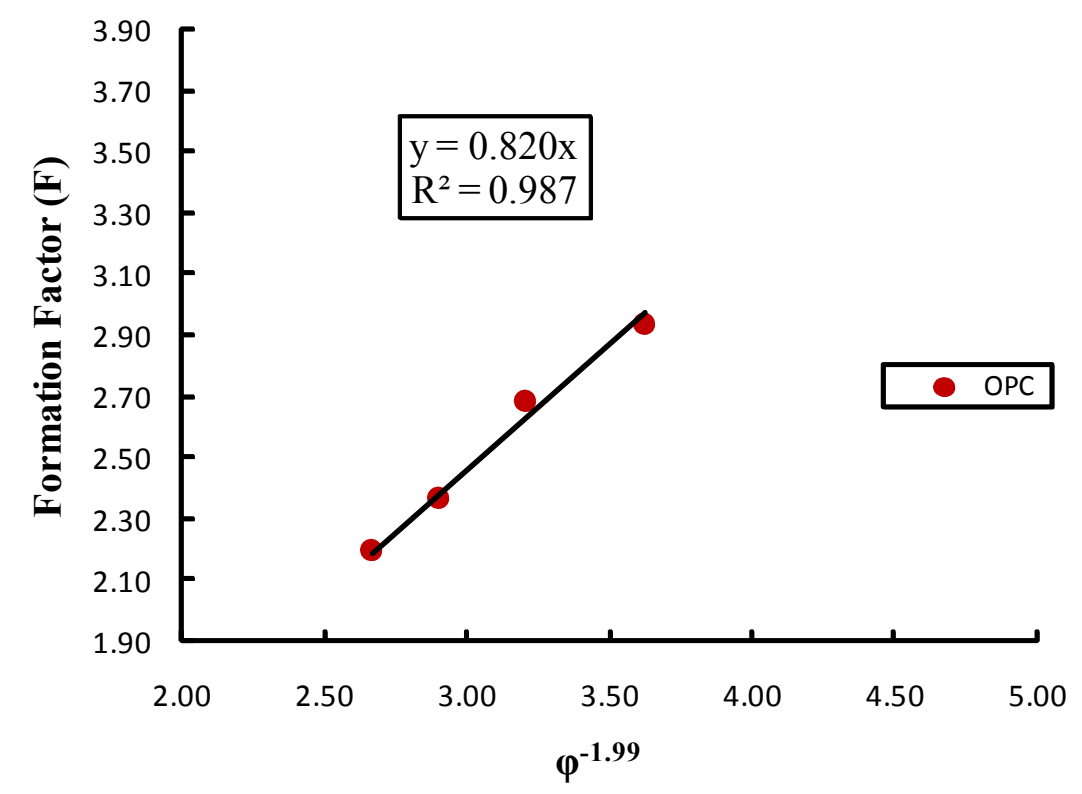

Figure 4.14. Regression analysis to calculate the tortuosity of ordinary portland cement paste when exponent $\mathrm{m}$ is equal to 1.99 . 


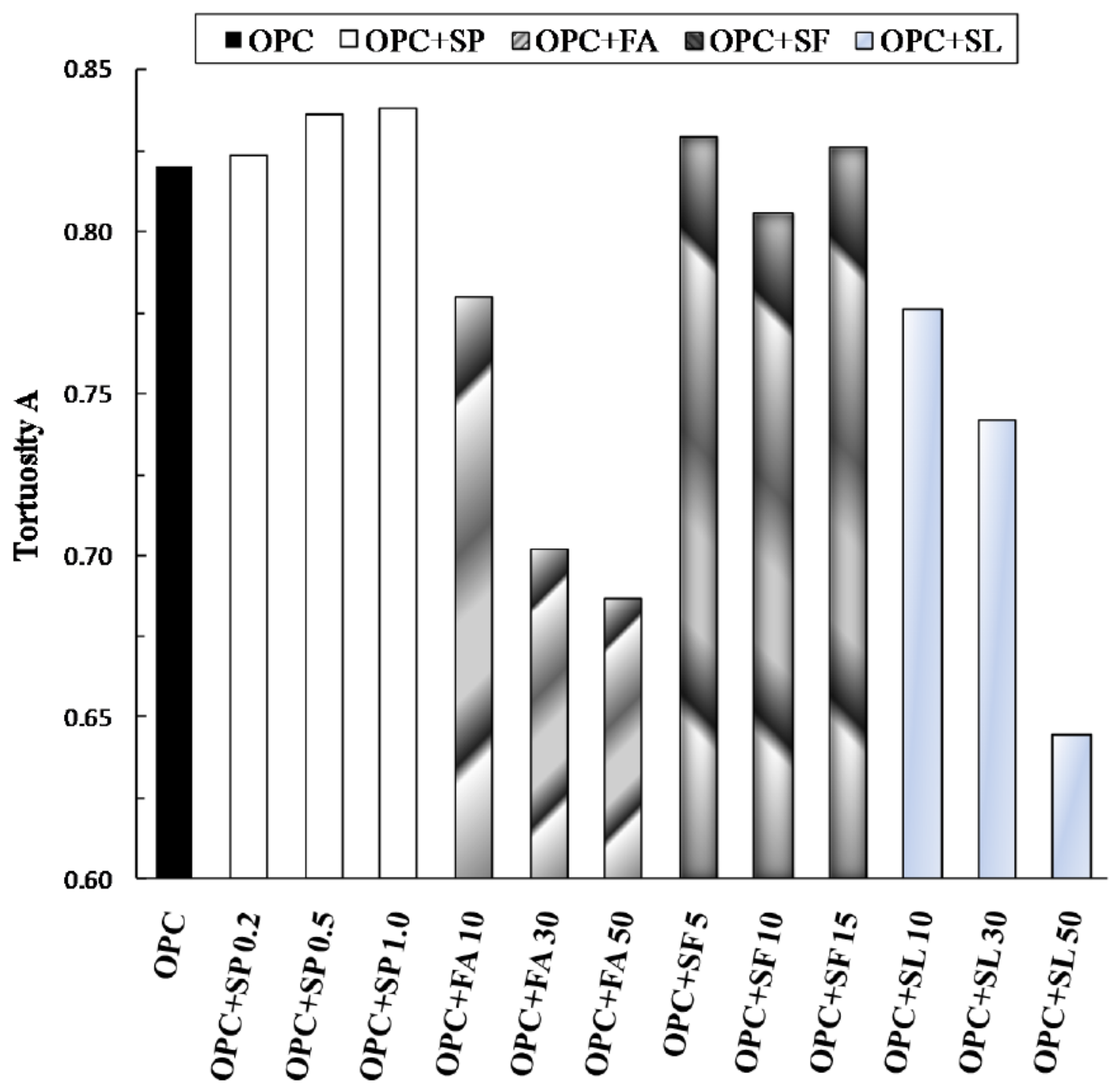

Figure 4.15. Tortuosity values of paste mixtures at fresh state.

Fig. 4.15 depicts variation of tortuosity values for different paste mixtures which shows the complexity of charge transfer in pore solution in the presence of various cementitious materials particles (solid phase). In other words, the greater the tortuosity value is, the more complex pathway for electrical charge to pass among the solid particles would be, which depends on the size, roundness and distribution of particles in pore solution. 
The OPC particles have the average size around $10 \mu \mathrm{m}$ and they are relatively spherical. Although the amount of superplasticizer dosage is relatively small (around $1 / 1000^{\text {th }}$ ) of the cement mass, it increases the tortuosity value of the paste (Fig. 4.15). The increase in tortuosity is attributed to the distribution effect of superplasticizer molecules on cement particles. The cement particles naturally have tendency to aggregate while mixing with water and thus always a small portion of them remains together. However, the superplasticizer physically separates them by yielding electrostatic repulsion when absorbed to the cement particles. Consequently, a more complex pathway for charge transfer is provided due to the better distribution of cement particles in water which results in higher tortuosity.

Fig. 4.15 illustrates that fly ash considerably reduced the tortuosity of the paste at fresh state. The average size of fly ash particles are about $15 \mu \mathrm{m}$ which are a bit larger than cement particles which result in less tortuosity, because less number of fly ash particles resist against the movement of ions compared to the cement particles. On the other hand, the considerable reduction of tortuosity in fly ash-incorporated pastes can be also related to the spherical shape of its particles (roundness); i.e., for two particles with the same size, the one which has more angular/broken shape yields higher tortuosity.

Silica fume particles average size is about $0.1 \mu \mathrm{m}$ which are in the order of $1 / 100^{\text {th }}$ of the cement particles. They naturally have tendency to aggregate and are more rounded than cement particles. The former effect tends to increase the tortuosity, while the latter effect decreases it. Fig. 4.15 shows that these two opposing effects compensate each other in a way that resultant tortuosity from addition of silica fume does not change significantly 
compared to that of cement particles and has no trend with increase in silica fume dosage. Therefore, although it is expected that because of very small size of silica fume particles, tortuosity increases, the roundness and aggregation effect decrease the tortuosity and none of them is dominant.

Fig. 4.15 demonstrates that the addition of the slag to the paste resulted in a large reduction in the tortuosity values. The average particle size of slag is around $45 \mu \mathrm{m}$ which is approximately 5 times larger than cement particles. Therefore, the number of slag particles per unit volume of the paste is about $1 / 25$ times less than that of the cement particles. As a result, the length of ion movement pathways decreases and consequently, the tortuosity significantly decreases in the slag incorporated pastes at the fresh state. Although the roundness of slag is less than the cement particles because of sudden cooling procedure in their production, this effect is not dominant and the tortuosity value is governed by the size of slag particles. The more the slag particles present in the paste mixture, the less the corresponding tortuosity would be.

Since porosity $\varphi$ directly depends on w/c through Eq. 4.5 and it is a variable independent from the ratio of supplementary cementitous materials $(R)$ and their density $\left(D_{S C M}\right)$, a simplified equation can be proposed from regression analysis on all the paste mixtures to correlate the formation factor $\mathrm{F}$ to the $\mathrm{w} / \mathrm{c}$ in the Archie's through Eq. 4.10. The results of this analysis are shown in Fig. 4.16. The separate set of results for each type of paste mixture are shown in Fig. B.3 in Appendix B. 


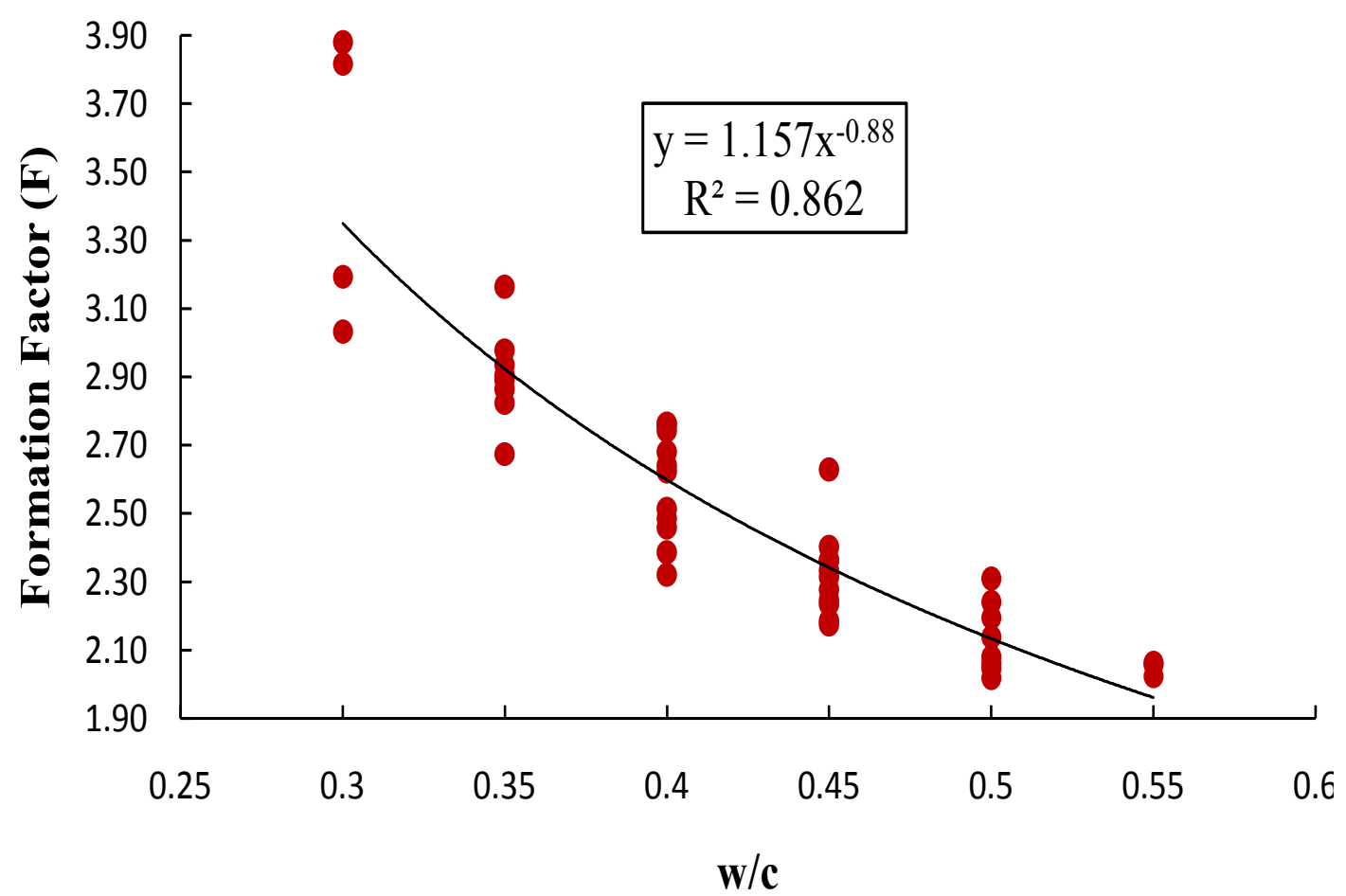

Figure 4.16. Formation factor versus $w / c$ ratio of fresh cement paste at $30^{\text {th }}$ minute of paste age considering all different paste mixtures

$$
F=A(w / c)^{-m}
$$

Fig. 4.16 illustrates $\mathrm{R}^{2}$ value of 0.86 for the F-w/c correlation and thus, even small improvement can be observed compared to that of F- $\varphi$ correlation; i.e., 0.84 is improved to 0.86 . Therefore, this simplified equation for all types of the paste provide a direct relationship between formation factor and w/c. Using this approach, there is no need to know the density of supplementary cementitious materials and their replacement ratio in the mixture to calculate $\mathrm{w} / \mathrm{c}$ from formation factor. 


\subsection{5. $\mathrm{pH}$ and pore solution conductivity correlation}

In order to study the variation of $\mathrm{pH}$ with pore solution conductivity, $\mathrm{pH}$ measurement was conducted on paste and its pore solution and the recorded data were then normalized to the reference temperature of $25^{\circ} \mathrm{C}$. To investigate the effect of $\mathrm{w} / \mathrm{c}$ on the $\mathrm{pH}$ of the pore solution, the data recorded at 30 minutes after mixing was considered. Besides, for the w/c of 0.45 the $\mathrm{pH}$ of the pore solution over time during the first two hours after mixing was monitored. In addition, to examine the variation in the recorded $\mathrm{pH}$ values, some repeating tests were performed. The average results for the w/c and time effect on the $\mathrm{pH}$ of the pore solution for the pastes with mid-dosage of SCMs and superplasticizer are shown in Fig. 4.17 and 4.18, respectively.

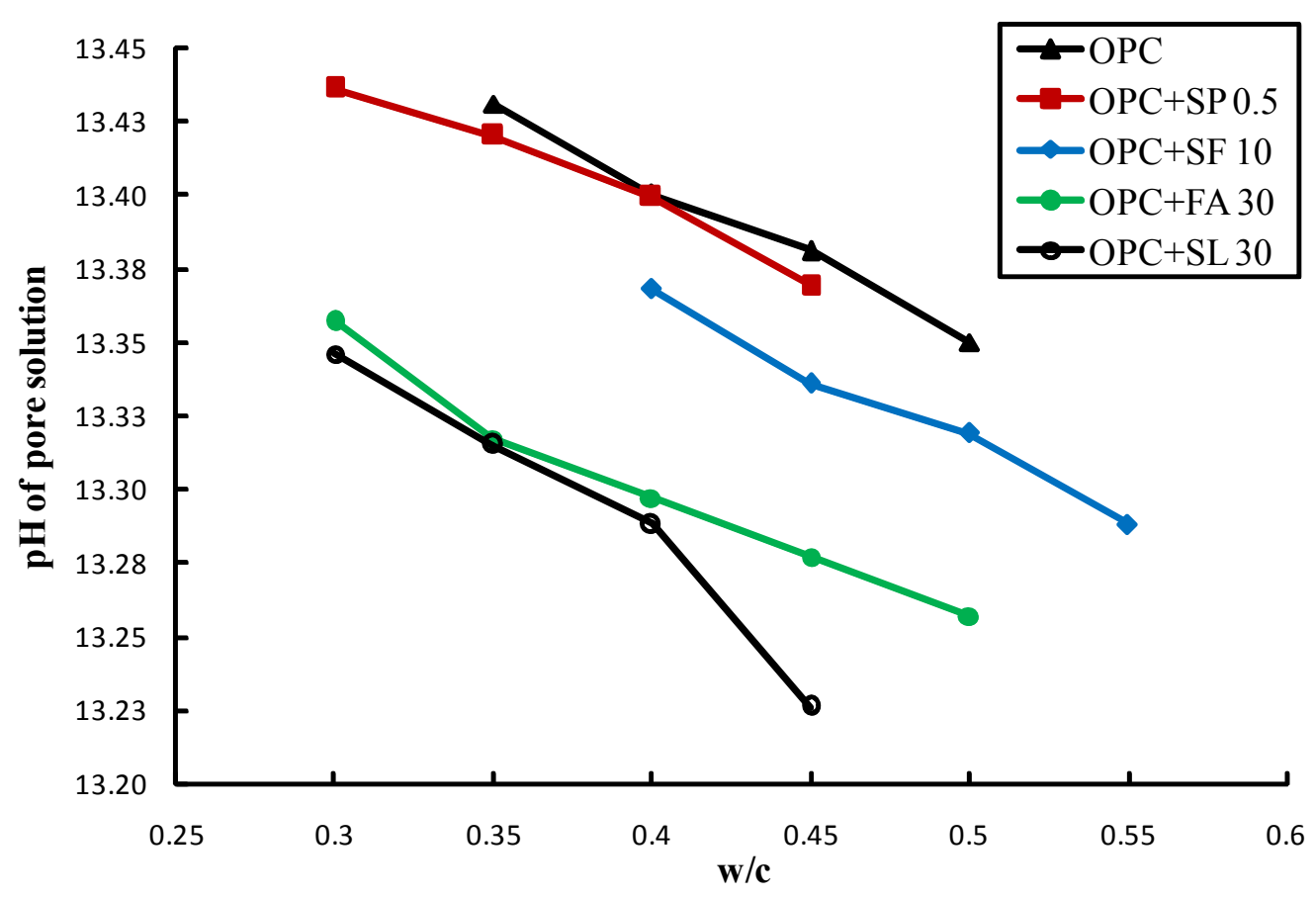

Figure 4.17. $\mathrm{pH}$ of the pore solution versus $\mathrm{w} / \mathrm{c}$ of the pastes at $30^{\text {th }}$ minute after mixing. 


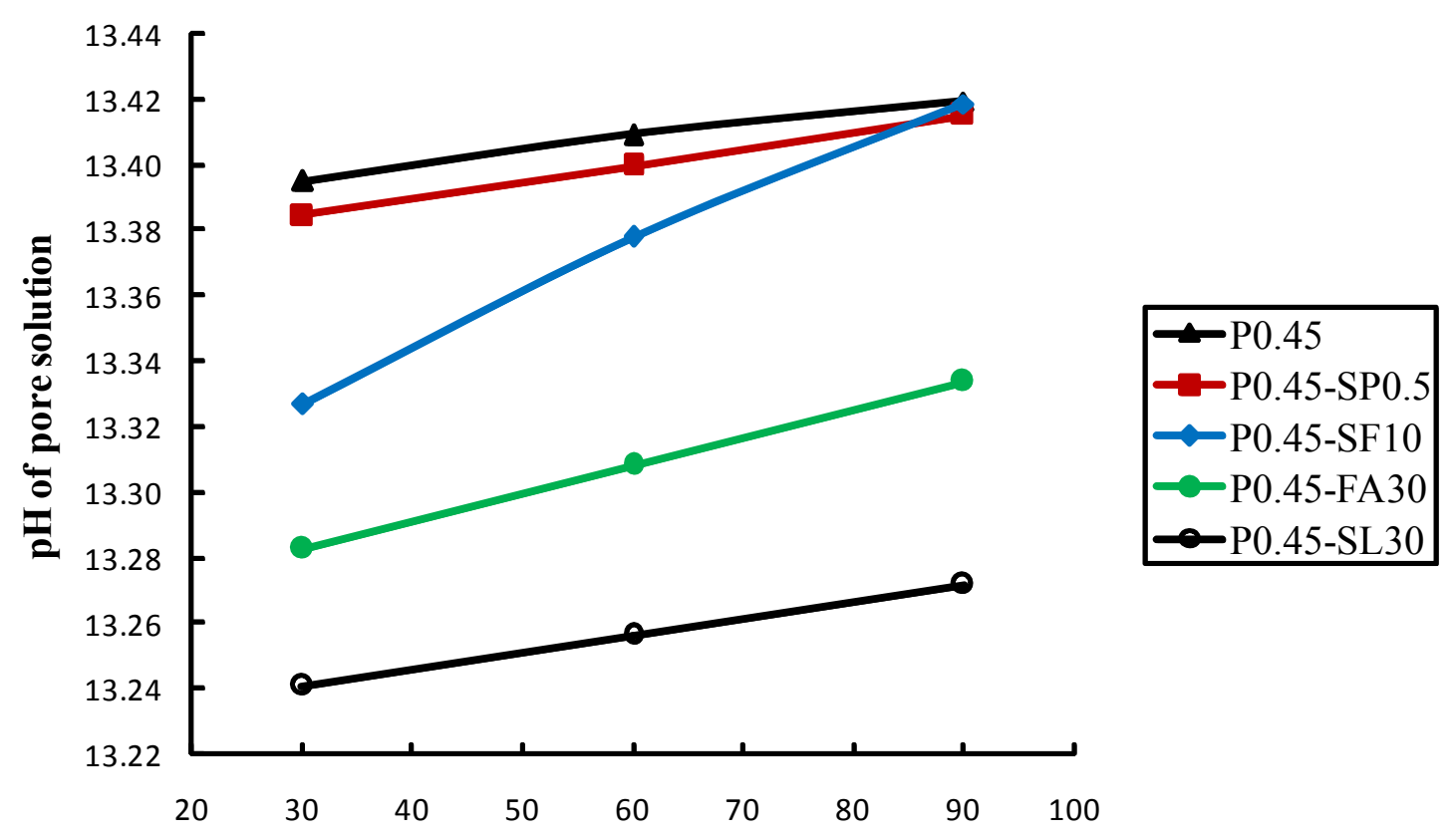

Time (Min)

Figure 4.18. $\mathrm{pH}$ development in pore solution with time for the pastes having w/c of 0.45 .

Fig. 4.17 shows that regardless of the type of the paste mixture, whether it contains admixture or not, increasing the $\mathrm{w} / \mathrm{c}$ of the paste reduces the $\mathrm{pH}$ value. The $\mathrm{pH}$ is the indication of the concentration of hydroxide ion $\left(\mathrm{OH}^{-}\right)$in the pore solution; i.e. the more the $\mathrm{OH}^{-}$concentration, the higher the corresponding $\mathrm{pH}$ of the pore solution. The reduction in the $\mathrm{pH}$ of the pore solution with increasing the $\mathrm{w} / \mathrm{c}$ is attributed to the concentration of hydroxide ion released in the pore solution. The amount of hydroxide ion per unit volume of pore solution, ion concentration (mol/l), decreases in the pastes with increasing the $\mathrm{w} / \mathrm{c}$. This results in the lower $\mathrm{pH}$ of associated pore solution. It is noted that the variation of $\mathrm{pH}$ versus $\mathrm{w} / \mathrm{c}$ follows the same trend as the pore solution conductivity $(\sigma)$ versus w/c as discussed earlier in Section 4.3.3. 
Fig. 4.18 reveals that the $\mathrm{pH}$ of pore solution over time for every paste mixture increases at the fresh state before the setting. Increase in the $\mathrm{pH}$ value indicates decrease in the $\mathrm{OH}^{-}$ concentration. It is most likely attributed to the further dissolution of alkali phases of cementitious materials in the pore solution.

Figures 4.17 and 4.18 also show that superplasticizer has no significant effect on the $\mathrm{pH}$, whereas supplementary cementitious materials such as silica fume, fly ash and slag reduce the $\mathrm{pH}$ of the pore solution. The magnitude of $\mathrm{pH}$ reduction is the highest for slag and the lowest for silica fume and fly ash $\mathrm{pH}$ reduction is in the middle. It was also observed that the $\mathrm{pH}$ and conductivity of the pore solution followed the same trend; i.e., when $\mathrm{pH}$ increased the pore solution conductivity increased and vice versa. The correlation between the pore solution conductivity and the associated $\mathrm{pH}$ were investigated in Fig. 4.19.

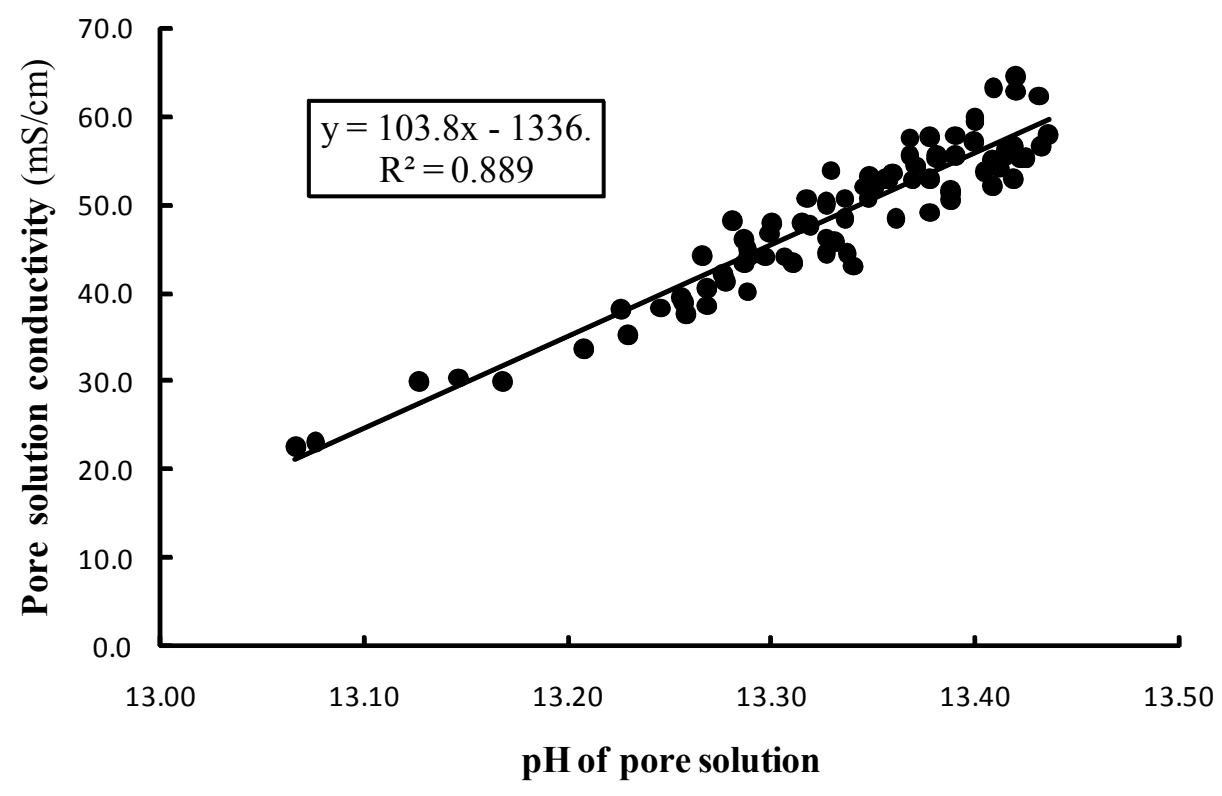

Figure 4.19. Pore solution conductivity and $\mathrm{pH}$ correlation at fresh cement paste. 


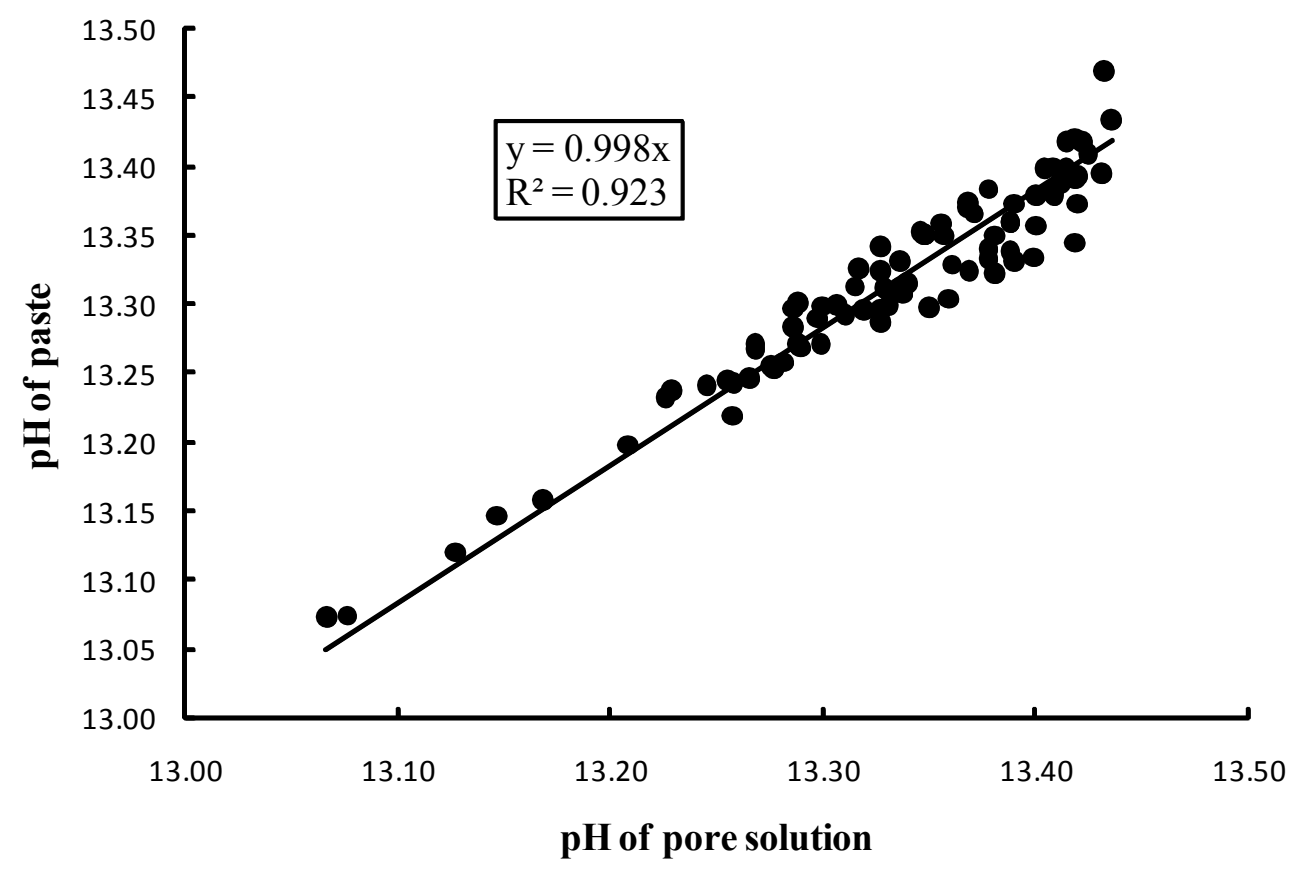

Figure 4.20. Paste and pore solution $\mathrm{pH}$ relationship in cement paste.

The trend line and $\mathrm{R}^{2}$ value of 0.89 in Fig. 4.19 indicates a strong correlation between the conductivity and $\mathrm{pH}$ of the pore solution in cement paste mixtures at fresh state. As a result, the pore solution conductivity can be estimated from $\mathrm{pH}$ via:

$\sigma=103.8(p H)-1336$

where $\sigma$ is the pore solution conductivity in $\mathrm{mS} / \mathrm{cm}$. This strong correlation between conductivity and $\mathrm{pH}$ in pore solution is attributed to the significant role of hydroxyl in the characteristics of pore solution [12]. The conductivity of the pore solution is directly related to the concentration of $\mathrm{OH}^{-}[12]$ and the $\mathrm{pH}$ of the pore solution is also directly proportional to the $\mathrm{OH}^{-}$concentration by definition as:

$$
p H=14+\log \left(C_{\mathrm{OH}^{-}}\right)
$$


where $\mathrm{C}_{\mathrm{OH}^{-}}$is hydroxide ion concentration in $\mathrm{mol} / \mathrm{l}$.

Although $\mathrm{pH}$ of the paste and that of the pore solution were not exactly the same values in the experiments, as seen in Fig. 4.20 their difference is negligible. Therefore, in practice, since the pore solution extraction is tiresome and time consuming, one can measure the $\mathrm{pH}$ of the paste with minimum amount of effort in a couple of seconds to determine the conductivity of the pore solution through Eq. 4.11.

\subsection{Summary}

The electrical charge transfer in liquid phase of cement paste (pore solution) directly depends on the ions concentration resulted from the dissolution of cementitious materials when reacted with water. At any time of hydration age during fresh state before initial setting time, the pore solution conductivity in all types of cement paste mixtures showed descending trend with w/c; i.e., the higher the w/c of the paste, the lower the corresponding pore solution conductivity. Because with w/c increase the concentration of ions in pore solution decreases, which corresponds to drop in the pore solution conductivity.

If the mass ratio of the liquid phase to that of the solid phase (i.e., w/c) is constant in a paste mixture, time has increasing effect on the pore solution conductivity. The rise in pore solution conductivity is attributed to the further progress of chemical reaction and dissolution of cementitious materials in water which results in higher concentration of ions in pore solution. Therefore, higher pore solution conductivity is resulted. 
The chemical and mineral admixtures can affect the pore solution conductivity. The results of this study pointed out that all types of additives reduce the pore solution conductivity. However, the reduction is more significant in slag, fly ash, silica fume and superplasticizer incorporated pastes, respectively.

On the other hand, paste electrical resistivity is a function of pore solution resistivity (chemical effect), water to cementitious material ratio as well as the size and distribution of the cementitious materials (physical effect); i.e., the higher pore solution resistivity and lower w/c in a paste results in higher paste resistivity. For each paste mixture as the pore solution resistivity decreases over time, the paste electrical resistivity drops in a same manner.

By increasing the $w / c$ in all paste mixtures initially a small drop in paste resistivity was observed; but it raised with further increase in w/c. This is most likely attributed to the opposing factors such as porosity, tortuosity and pore solution resistivity that all change when the $w / c$ increases.

Furthermore, the formation factor $\mathrm{F}$ which is the ratio of paste resistivity to that of pore solution exhibits descending trend with volumetric fraction of pore solution in the paste matrix (i.e., porosity $\varphi$ ). Therefore, the relationship between the formation factor and porosity can be defined through Archie's law (Eq. 4.1). The magnitude of formation factor is also affected by an experimental constant called tortuosity (A) which is related to the size and distribution of solid particles in the pore solution. The tortuosity increases in pastes with superplasticizer, whereas slag and fly ash incorporated pastes have reducing effect. Silica fume has almost the same tortuosity as the OPC pastes. 
The $\mathrm{pH}$ index shows a strong correlation with the pore solution conductivity in paste mixtures at fresh state; i.e., as the $\mathrm{pH}$ of pore solution increases the pore solution conductivity increases and vice versa. Therefore, the $\mathrm{pH}$ measurement of the paste, which is much faster and easier than that of the pore solution, can be considered as an alternative method to estimate the conductivity of the pore solution. 


\section{Numerical Modeling to estimate the conductivity of pore solution}

\subsection{Introduction}

Within a cementitious paste, the pore solution can be regarded as the conductive component while the solid cementitious materials, in comparison, might be assumed to have no electrical conductivity. Therefore, if the electrical conductivity of the pore solution is known, the electrical resistivity of the paste can be determined through the formation factor. Although the direct measurement of pore solution conductivity is possible through pore solution extraction processes, this is not a practical tool for aged pastes, particularly for those specimens with low w/c. Modeling pore solution conductivity, on the other hand, can be used to mitigate this issue. This chapter provides the modeling approaches that are used in this thesis.

\subsection{Concentration of ionic species in pore solution}

A typical pore solution from paste or mortar (or concrete) contains a wide range of ions including $\mathrm{Na}^{+}, \mathrm{K}^{+}, \mathrm{OH}^{-}, \mathrm{Ca}^{2+}$, and $\mathrm{SO}_{4}{ }^{2-}[62]$. Pore solution extraction studies show that typically the concentration of $\mathrm{Na}^{+}, \mathrm{K}^{+}$and $\mathrm{OH}^{-}$are orders of magnitude larger than other ionic species such as $\mathrm{Ca}^{2+}$, and $\mathrm{SO}_{4}{ }^{2-}[12]$, and in most cases these minor species can be ignored for the purpose of electrical conductivity predictions. In some cases, however, it has been shown that $\mathrm{SO}_{4}{ }^{2-}$ concentration can be influential [63]; therefore, in this study its effect on the total pore solution conductivity was also taken into account and was not ignored during modeling. Struble [63], based on an experimental study on series of mortars ranging in age from 1 to 28 weeks, proposed the following relationship to 
estimate $\mathrm{SO}_{4}{ }^{2-}$ concentration in cement pore solution from the known concentrations of $\mathrm{Na}^{+}$and $\mathrm{K}^{+}$:

$C_{\mathrm{SO}_{4}^{2-}}=0.06\left[C_{\mathrm{Na}^{+}}+C_{\mathrm{K}^{+}}\right]^{2}$

where $\mathrm{C}_{\mathrm{SO}_{4}}{ }^{2-}, \mathrm{C}_{\mathrm{Na}^{+}}$, and $\mathrm{C}_{\mathrm{K}^{+}}$, are concentrations of $\mathrm{SO}_{4}{ }^{2-}, \mathrm{Na}^{+}$, and $\mathrm{K}^{+}$, respectively.

Following this, using the charge balance of the system, the $\mathrm{OH}^{-}$concentration, $\mathrm{C}_{\mathrm{OH}^{-}}$, can be calculated via:

$C_{\mathrm{OH}^{-}}=C_{\mathrm{Na}^{+}}+C_{\mathrm{K}^{+}}-\left(2 \times C_{\mathrm{SO}_{4}^{2-}}\right)$

Taylor [37], using the experimental data from previous works and characterizing the hydration behavior of OPC and OPC plus fly ash pastes, mortars and concretes after 1 day of mixing, proposed a model to predict $\mathrm{Na}^{+}$and $\mathrm{K}^{+}$concentration in the corresponding pore solution. The minimum data required for Taylor's model are the total alkali $\left(\mathrm{Na}_{2} \mathrm{O}\right.$ and $\left.\mathrm{K}_{2} \mathrm{O}\right)$ contents in the OPC and fly ash, the age in days, and the paste properties (i.e., water, cement and fly ash contents). The principle used in the model comes from the mass balance relationship: the amount of released alkali cation, $\mathrm{m}_{\mathrm{r}}$, at any time of hydration equals to the quantity of the portion distributed and dissolved in the pore solution, $\mathrm{m}_{\mathrm{d}}$, plus the portion that is taken up by hydration products, $\mathrm{m}_{\mathrm{p}}$ [37]:

$m_{r}=m_{d}+m_{p}$

where all units are presented in mmoles. Eq. (5.3) can be rewritten as: 
$C=\frac{m_{r}}{V+(b \times P)}$

where $\mathrm{C}$ is the concentration of an ionic species $(\mathrm{mol} / \mathrm{l}) ; \mathrm{V}$ is the volume of pore solution $\left(\mathrm{cm}^{3}\right) ; \mathrm{P}$ is dimensionless ratio of calcium quantity (mmole) in relevant hydration products (C-S-H and AFm phases) that are able to take up alkali cations at any time $\mathrm{t}$, denoted by CRP, to the calcium content at complete hydration, $\mathrm{CRP}_{\infty}$, which was experimentally shown to be 791 mmoles for $100 \mathrm{~g}$ of OPC [37]; and b $\left(\mathrm{cm}^{3}\right)$ is a constant called "binding factor" representing the potential of $\mathrm{Na}^{+}$and $\mathrm{K}^{+}$to be taken up by CRP's. The values for $\mathrm{Na}^{+}$and $\mathrm{K}^{+}$were empirically determined $31.0 \mathrm{~cm}^{3}$ and $20.0 \mathrm{~cm}^{3}$, respectively [37].

Based on the equation presented by Dalziel and Gutteridge for the hydration rates of all four major clinker phases (i.e., alite or $\mathrm{C}_{3} \mathrm{~S}$, belite or $\mathrm{C}_{2} \mathrm{~S}$, ferrite or $\mathrm{C}_{4} \mathrm{AF}$, and aluminate or $\mathrm{C}_{3} \mathrm{~A}$ ) [64], Taylor [37] proposed the following exponential function with respect to time to estimate the reacted fractions of cementitious materials (OPC and fly ash) considering different contribution of major clinker phases and hydration products to take up the alkali cations:

$F=1-\exp \left[-k_{2}\left(t-k_{3}\right)^{k_{1}}\right]$

where $\mathrm{F}$ is the fraction of each major clinker phase reacted at time $\mathrm{t}$ (in days); and $\mathrm{k}_{1}, \mathrm{k}_{2}$ and $\mathrm{k}_{3}$ are the empirical constants which gave the best agreement with Dalziel and Gutteridge's data [64]. The pore solution volume at any time per $100 \mathrm{~g}$ of cementitious materials, $\mathrm{V}$, can be predicted as: 
$V=(w / c \times 100)-V_{b}$

where $\mathrm{w} / \mathrm{c}$ is the water-to-cementitious materials ratio; and $\mathrm{V}_{\mathrm{b}}$ is the volume $\left(\mathrm{cm}^{3}\right)$ of bound water in the hydration products structure. $\mathrm{V}_{\mathrm{b}}$ at complete hydration was approximated to be $31.6 \mathrm{~g}$ per $100 \mathrm{~g}$ of OPC [65].

Table 5.1 provides the total contents of $\mathrm{Na}_{2} \mathrm{O}$ and $\mathrm{K}_{2} \mathrm{O}$ in each clinker phase, determined by scanning electron microscopy [66]; Table 5.2 provides the values of $\mathrm{k}_{1}, \mathrm{k}_{2}$ and $\mathrm{k}_{3}$ as reported by Taylor [37].

Table 5.1. Total contents of $\mathrm{Na}_{2} \mathrm{O}$ and $\mathrm{K}_{2} \mathrm{O}$ in four major clinker phases of OPC.

\begin{tabular}{|c|cccc|}
\cline { 2 - 5 } \multicolumn{1}{c|}{} & $\mathbf{C}_{\mathbf{3}} \mathbf{S}$ & $\mathbf{C}_{2} \mathbf{S}$ & $\mathbf{C}_{\mathbf{3}} \mathbf{A}$ & $\mathbf{C}_{4} \mathbf{A F}$ \\
\hline $\mathbf{N a}_{\mathbf{2}} \mathbf{O}$ content (\% of mass) & 0.17 & 0.27 & 1.05 & 0.06 \\
$\mathbf{K}_{\mathbf{2}} \mathbf{O}$ content (\% of mass) & 0.14 & 0.79 & 0.97 & 0.07 \\
\hline
\end{tabular}

Table 5.2. Empirical values of constants for OPC hydration at any age in days to be used in Eq. 5.5 [37].

\begin{tabular}{|l|llllll|}
\cline { 2 - 7 } \multicolumn{1}{c|}{} & $\mathbf{C}_{\mathbf{3}} \mathbf{S}$ & $\mathbf{C}_{\mathbf{2}} \mathbf{S}$ & $\mathbf{C}_{\mathbf{3}} \mathbf{A}$ & $\mathbf{C}_{\mathbf{4}} \mathbf{A F}$ & $\mathbf{V}_{\mathbf{b}}{ }^{*}$ & $\mathbf{P}^{* *}$ \\
\hline $\mathbf{k}_{\mathbf{1}}$ & 0.25 & 0.46 & 0.28 & 0.26 & 0.25 & 0.25 \\
$\mathbf{k}_{\mathbf{2}}$ & 0.70 & 0.12 & 0.77 & 0.55 & 0.69 & 0.56 \\
$\mathbf{k}_{\mathbf{3}}$ & 0.90 & 0.00 & 0.90 & 0.90 & 0.90 & 0.90 \\
\hline
\end{tabular}

(*) Fraction of $31.6 \mathrm{~g} / 100 \mathrm{~g}$ OPC

(**) CRP / CRP 
Table 5.3. Modified empirical values of constants for OPC plus fly ash pastes in Eq.

\begin{tabular}{|c|ccccc|}
\cline { 2 - 6 } \multicolumn{1}{c|}{} & $\mathbf{C}_{\mathbf{3}} \mathbf{S}$ & $\mathbf{\mathbf { C } _ { 2 }} \mathbf{S}$ & $\mathbf{C}_{\mathbf{3}} \mathbf{A}$ & $\mathbf{C}_{\mathbf{4}} \mathbf{A F}$ & $\mathbf{V}_{\mathbf{b} \mathbf{1}} *$ \\
\hline $\mathbf{k}_{\mathbf{1}}$ & 0.25 & $0.46-0.002 * \mathrm{R}$ & 0.28 & 0.26 & 0.25 \\
$\mathbf{k}_{\mathbf{2}}$ & $0.7+0.008 * \mathrm{R}$ & $0.12-0.0007 * \mathrm{R}$ & $0.77+0.008 * \mathrm{R}$ & $0.55+0.012 * \mathrm{R}$ & $0.69+0.008 * \mathrm{R}$ \\
$\mathbf{k}_{\mathbf{3}}$ & 0.90 & 0.00 & 0.90 & 0.90 & 0.90 \\
\hline
\end{tabular}

(*) Fraction of $31.6 \mathrm{~g} / 100 \mathrm{~g}$ OPC

When OPC is incorporated with fly ash with a replacement ratio $\mathrm{R}$, some modifications should be taken into account. Studies on OPC plus fly ash pastes showed that fly ash accelerates $\mathrm{C}_{3} \mathrm{~S}, \mathrm{C}_{3} \mathrm{~A}$ and $\mathrm{C}_{4} \mathrm{AF}$ hydration, while delaying that of $\mathrm{C}_{2} \mathrm{~S}$ [64]. Taylor [37] applied this effect on $\mathrm{k}_{1}$ and $\mathrm{k}_{2}$ values in Eq. 5.5 as shown in Table 5.3.

Furthermore, the volume of bound water $\mathrm{V}_{\mathrm{b}}$ in Eq. (5.6) has two components if paste contains fly ash: the volume of bound water due to cement hydration, $\mathrm{V}_{\mathrm{b} 1}$, which is given in Table 5.3, and the volume of bound water of pozzolanic (fly ash) reactions, $\mathrm{V}_{\mathrm{b} 2}$, which is defined as a fraction of fly ash reacted in $100 \mathrm{~g}$ of cementitious materials via:

$V_{b 2}=0.17 \mathrm{~A}$

where $\mathrm{A}$ is the amount of fly ash $(\mathrm{g})$ reacted in $100 \mathrm{~g}$ of cementitious materials.

Additionally, the dimensionless ratio $\mathrm{P}$, which is the ratio of the calcium in hydration products at any hydration age to that of complete hydration, is modified as follows:

$$
P=\left(1-\exp \left[-k_{2}(T-0.9)^{0.25}\right]\right) \times R^{0.09}
$$


where $\mathrm{k}_{2}$ is given via:

$k_{2}=0.56-(0.0043 \times R)$

In addition, the specific abilities of C-S-H and AFm phases to absorb the alkali ions when incorporated with fly ash is considered by introducing constant $b^{\prime}$ [37] based on Bhatty and Greening's study [67] which provided the alkali ions concentration via:

$C=\frac{m_{r}}{V+(b \times P)+\left(b^{\prime} \times A\right)}$

where $\mathrm{b}^{\prime}$ values in $\mathrm{cm}^{3} / \mathrm{g}$ were determined empirically to be 3.0 and 3.3 for $\mathrm{Na}^{+}$and $\mathrm{K}^{+}$, respectively. Assuming that only the glass fraction of the fly ash, G, is reactive and the crystalline constituents including mostly quartz and iron oxides are inert, Taylor [37] proposed Eq. 5.11 which was in agreement with the experimental data for fly ash reacted quantity from previous works $[35,64,68]$.

$A=\left(1-\exp \left[-k_{2} T^{k_{1}}\right]\right) \times G \times R$

where $\mathrm{k}_{1}$ and $\mathrm{k}_{2}$ are given via:

$k_{1}=0.585-(0.0005 \times G \times R)$

$k_{2}=0.043-(0.0005 \times G \times R)$

The calculations are defined per $100 \mathrm{~g}$ of binder (OPC plus fly ash). Thus, the fraction F which is obtained from Eq. 4.5 for OPC hydration (Table 4.2), should be multiplied by 
(1-R/100) to take into account the effect of the reduced proportion of the OPC in the paste.

The $\mathrm{m}_{\mathrm{r}}$ value used in Eq. 5.10 should be expressed in mmole for $\mathrm{Na}^{+}$and $\mathrm{K}^{+}$. Accordingly, when the total weight of reacted $\mathrm{Na}_{2} \mathrm{O}$ and $\mathrm{K}_{2} \mathrm{O}$ are calculated in $\mathrm{g}$, their amount can be converted in mmole using the associated molar mass. Then, based on the stoichiometry of their balanced chemical reaction with water molecules, as shown in Eq. 5.14 and 5.15, the final released amount of alkali cations $\left(\mathrm{Na}^{+}\right.$and $\left.\mathrm{K}^{+}\right)$in pore solution in mmole is twice of that of obtained $\mathrm{Na}_{2} \mathrm{O}$ and $\mathrm{K}_{2} \mathrm{O}$.

$\mathrm{Na}_{2} \mathrm{O}+\mathrm{H}_{2} \mathrm{O} \rightarrow 2 \mathrm{Na}^{+}+2 \mathrm{OH}^{-}$

$\mathrm{K}_{2} \mathrm{O}+\mathrm{H}_{2} \mathrm{O} \rightarrow 2 \mathrm{~K}^{+}+2 \mathrm{OH}^{-}$

\subsection{Calculation of pore solution conductivity}

The pore solution in a cement paste acts as an electrolyte whose electrical conductivity depends on its ionic concentration (i.e., the greater the concentration of the ions, the higher the corresponding total conductivity of pore solution). However, the relationship between the two was shown to be non-linear [39]. Although a number of accurate equations exist for predicting equivalent conductivity, $\lambda_{\mathrm{i}}$, which is defined as conductivity per unit concentration for each ionic species, these relationships are rather complex with multiple parameters and coefficients that are difficult to predict [39]. However, Eq. 2.14 developed for $25^{\circ} \mathrm{C}[39]$ is rather practical. 
Table 5.4. Equivalent conductivity at infinite dilution $\lambda^{\circ}$ and conductivity coefficients $G$ at $25^{\circ} \mathrm{C}$

\begin{tabular}{|ccc|}
\hline Ionic species & $\mathbf{z} \lambda^{\circ}\left({\left.\mathrm{S} . \mathrm{cm}^{2} / \mathrm{mol}\right)}\right.$ & $\mathbf{G}(\mathrm{mol} / 1)^{-1 / 2}$ \\
\hline $\mathrm{Na}^{+}$ & 50.1 & 0.733 \\
$\mathrm{~K}^{+}$ & 73.5 & 0.548 \\
$\mathrm{SO}_{4}^{2-}$ & 79.0 & 0.877 \\
$\mathrm{OH}^{-}$ & 198.0 & 0.353 \\
$\mathrm{Ca}^{+2}$ & 59.0 & 0.771 \\
$\mathrm{Cl}^{-}$ & 76.4 & 0.548 \\
\hline
\end{tabular}

where $I_{M}$ is the ionic strength of pore solution and is defined by Eq. 2.15; the values for $\mathrm{G}_{\mathrm{i}}$ [12] and $\lambda^{\circ}$ [39] (defined in Eq. 2.14) at $25^{\circ} \mathrm{C}$ were determined from the best agreement between calculated data and experimental ones, as shown in Table 5.4.

The total electrical conductivity of pore solution, $\sigma_{\text {cal }}$, can be expressed as weighted sum of equivalent conductivity for each ionic species [49]:

$\sigma_{c a l}=\sum z_{i} C_{i} \lambda_{i}$

where $\mathrm{z}_{\mathrm{i}}$ and $\mathrm{C}_{\mathrm{i}}$ are the valence and molar concentration of ionic species, respectively.

Based on Eq. 5.16, the most significant ionic species contributing to conductivity is $\mathrm{OH}^{-}$ because of its rather large equivalent conductivity $\left(198.0 \mathrm{~S} . \mathrm{cm}^{2} / \mathrm{mol}\right)$ as well as its high concentration in the pore solution of cement-based materials. $\mathrm{K}^{+}$and $\mathrm{Na}^{+}$have rather high solubility in pore solution which results in rather high corresponding concentration even at fresh state [12]. Accordingly, they also contribute to the total pore solution 
conductivity. The $\mathrm{Ca}^{2+}$ concentration was shown [38] to be around $.001 \mathrm{~mol} / 1$ whose conductivity can be neglected. The $\mathrm{SO}_{4}{ }^{2-}$ concentration can be estimated by Eq. 5.1 which gives negligible contribution to the total conductivity: i.e., around $1 \%$ of total pore solution conductivity. Therefore, if the concentrations of ions are known (either experimentally measured or using the proposed model such as the one discussed in Section 5.2), we only need to consider the contribution of $\mathrm{OH}^{-}, \mathrm{Na}^{+}$and $\mathrm{K}^{+}$as dominant ionic species for prediction of the pore solution conductivity using Eq. 5.16. The conductivity of very-low-volume pore solutions can be indirectly calculated from the conductivity of the diluted pore solution. During the dilution process since only distilled water is added to the pore solution, the number of ions are constant; thus, the concentration ratio of pore solution after dilution to that of before dilution would be inversely proportional to the volume ratios as follows:

$\frac{C_{1}}{C_{2}}=\frac{V_{2}}{V_{1}}$

where $\mathrm{C}_{1}$ and $\mathrm{C}_{2}$ are ions concentration (mol/l) before and after dilution, respectively; and $\mathrm{V}_{1}$ and $\mathrm{V}_{2}$ are solutions volume (1) before and after dilution, respectively.

\subsection{Proposed model for conductivity of pore solution in fresh state}

\subsubsection{Fresh state vs. hardened state}

Since the pore solution of cement-based materials can be regarded as electrolyte from electrical point of view, its electrical conductivity, $\sigma_{\mathrm{p}}$, is influenced by containing ions including $\mathrm{OH}^{-}, \mathrm{Na}^{+}, \mathrm{K}^{+}, \mathrm{SO}_{4}{ }^{2-}$ and $\mathrm{Ca}^{2+}$. Therefore, the concentration of these species 
determines the total electrical conductivity of pore solution. The concentration of each ionic species, C, is defined as its quantity (mole) per unit volume (l) of cement pore solution which is mainly water and can contain small fraction of other liquid admixtures such as superplasticizer. These ionic species are produced from cementitious materials after they are mixed with water. Thus, at any hydration age, the reacted portion of cementitious materials is the source of ions released in pore solution.

As discussed in Section 5.2, three mechanisms are considered to characterize the scenario occurs in hydration of the cementitous materials resulting in ions dissolution in the pore solution: (1) release of ions which are produced from cementitious materials and depends on the total associated constituent compounds such as $\mathrm{Na}_{2} \mathrm{O}$ and $\mathrm{K}_{2} \mathrm{O}$ in the chemical composition and their fraction reacted at any particular time, $\mathbf{m}_{\mathbf{r}} ;(2)$ a portion of released ions that is dissolved in the pore solution that contributes to the electrical charge transport, and consequently the conductivity of pore solution, $\mathbf{m}_{\mathbf{d}}$; (3) a portion of released ions that is taken up by hydration products including C-S-H and Afm phase both from OPC reaction and pozzolanic reactions, $\mathbf{m}_{\mathbf{p}}$ (adsorbed by hydration products structure and no longer exist in the pore solution). Consequently, this mechanism reduces the quantity of released ions which are able to contribute to the conductivity of pore solution.

In the hardened state, after setting, at any hydration age, all three mechanisms above contribute to the quantity of ions in the pore solution because the solidification initiates after initial setting, and as a result, the hydration products are formed and increase as cement paste ages. The volume of pore solution on the other hand, decreases as hydration 
products are formed because a fraction of water is trapped in hydration products structure as bound water, $\mathrm{V}_{\mathrm{b}}$. Therefore, at any specific time after mixing the materials, the quantity of ions dissolved in pore solution, should be calculated considering the adsorption effect of hydration products. Additionally, the pore solution volume should also be modified from the initial liquid phase volume presents at the time of mixing to take into account the bound water effect.

In the fresh state and before setting (approximately first 2 hours), no solidification occurs in the mixture; thus, all three mechanisms that are described above are not applicable. The moment liquid content is mixed with cementitious materials, the soluble compounds dissolve in pore solution. Releasing of ions occurs with an accelerated rate only during the first few minutes and continues during the paste age with a decelerated rate up to the initial setting [14]. Since no hydration product is formed during this period, no adsorption of ions takes place, and thus, all the released ions from cementitious materials are present in the pore solution. Consequently, they all contribute to the conductivity. On the other hand, the pore solution volume does not decrease during the fresh state and has the same volume as indicated in the mixture proportions because no water is trapped yet as bound water in hydration products.

\subsubsection{Proposed Model for pore solution conductivity at fresh state}

The model which is proposed in this section is intended to be used for fresh state of paste within the first two hours of mixing. It predicts the conductivity of pore solution by using the concepts discussed in Sections 5.2 and 5.3. In order to cover the effect of most

commonly used supplementary cementitious materials used in industry, four different 
types of pastes were considered (i.e., cementitious materials included OPC, fly ash, silica fume, and slag); and for the liquid phase, the effect of superplasticizer was taken into account.

Since the chemical composition of cementitious materials used in the concrete varies, it should be considered in the model. Previous studies $[12,37]$ showed that the dominant ionic species in the pore solution conductivity of the cement-based materials are $\mathrm{OH}^{-}$, $\mathrm{Na}^{+}$and $\mathrm{K}^{+}$. Therefore, all data required for this model are the mixture proportions, $\mathrm{Na}_{2} \mathrm{O}$ and $\mathrm{K}_{2} \mathrm{O}$ contents of cementitious materials, the relative hydrodynamic viscosity of superplasticizer to water which is denoted by $\mu_{\mathrm{SP}} / \mu_{\mathrm{W}}$ in this thesis, and the time $t$ (minutes) at which the conductivity of pore solution is estimated.

The model has two major steps as follows: the first step that predicts the concentration of dominant ions (i.e., $\mathrm{Na}^{+}$and $\mathrm{K}^{+}, \mathrm{OH}^{-}$, and $\mathrm{SO}_{4}{ }^{2-}$ ) in total pore solution conductivity; the second step that estimates the corresponding conductivity of pore solution from ionic concentration obtained at the first step.

The mass balance principle was employed to find the amount of released alkali cations $\left(\mathrm{Na}^{+}\right.$and $\left.\mathrm{K}^{+}\right)$from $\mathrm{Na}_{2} \mathrm{O}$ and $\mathrm{K}_{2} \mathrm{O}$ contents of cementitious materials. In the fresh state, since no hydration product is formed to take up the alkali cations, the same amount of released ions would dissolve in the pore solution as follows:

$m_{r}=m_{d}$

Assuming the uniform distribution of ions in the pore solution, ionic concentration of species, C, can be obtained via: 
$C=\frac{m_{r}}{V}$

where $\mathrm{m}_{\mathrm{r}}$ is the released amount of alkali cation in the pore solution (mmole) and $\mathrm{V}$ is the volume of pore solution $\left(\mathrm{cm}^{3}\right)$. When the $\mathrm{Na}^{+}$and $\mathrm{K}^{+}$concentrations are found using Eq. 5.19, the $\mathrm{SO}_{4}{ }^{2-}$ and $\mathrm{OH}^{-}$concentrations can be calculated using Eq. 5.1 and Eq. 5.2, respectively.

The determination of $\mathrm{m}_{\mathrm{r}}$ for alkali cations from different cementitious materials is the most challenging step because it should consider the chemical reactions associated with all different cementitious materials dissolving in liquid phase and their various rates of reactions based on their initial $\mathrm{Na}_{2} \mathrm{O}$ and $\mathrm{K}_{2} \mathrm{O}$ contents. Adding supplementary cementitious materials would delay dissolution of ions in pore solution [13] and consequently reduces $\mathrm{m}_{\mathrm{r}}$ in OPC pastes containing fly ash, silica fume or slag. Besides, based on literature $[14,35,37]$, solid particles reactions with water and consequently the pore solution conductivity has a sharp increase with time within the first minutes after mixing the materials, while it shows pretty smooth increase afterward until initial setting time. This variation with time suggests an exponential function for reacted fraction of cementitious materials. Furthermore, analytical model should satisfy the condition that the $\mathrm{m}_{\mathrm{r}}$ amount at time zero equals zero, because there is no significant ion in the liquid content at the moment the mixing initiates. Accordingly, the exponential function $\mathrm{F}$ was developed with respect to time $\mathrm{t}$ as follows:

$$
F=1-\exp \left[-k_{2}^{\prime} t^{k_{1}^{\prime}}\right]
$$


where $\mathrm{F}$ is the reacted fraction of each specific cementitious material; $t$ is the time in minutes; and $\mathrm{k}_{1}^{\prime}$ and $\mathrm{k}_{2}^{\prime}$ are the empirical constants.

To perform the calculation per $100 \mathrm{~g}$ of total cementitious materials, the replacement percentage associated with each cementitious material was used. OPC, fly ash, silica fume, and slag replacement per $100 \mathrm{~g}$ of total cementitous materials were denoted by $\mathrm{R}_{\mathrm{OPC}}, \mathrm{R}_{\mathrm{FA}}, \mathrm{R}_{\mathrm{SF}}$, and $\mathrm{R}_{\mathrm{SL}}$, respectively. Therefore, the total $\mathrm{m}_{\mathrm{r}}$ of $\mathrm{Na}^{+}$or $\mathrm{K}^{+}$released into pore solution in mmole was obtained using the reacted fraction $\mathrm{F}$ of total $\mathrm{Na}_{2} \mathrm{O}$ or $\mathrm{K}_{2} \mathrm{O}$ contents from chemical composition analysis as follows:

$$
m_{r}=\frac{2 \times 10^{3}}{M}\left[F_{O P C} m p_{O P C} R_{O P C}+F_{F A} m p_{F A} R_{F A}+F_{S F} m p_{S F} R_{S F}+F_{S L} m p_{S L} R_{S L}\right]
$$

where $\mathrm{M}$ is the molar mass of alkali (i.e., $\mathrm{Na}_{2} \mathrm{O}$ or $\mathrm{K}_{2} \mathrm{O}$ ) in $\mathrm{mol} / \mathrm{g}$; $\mathrm{F}$ is the reacted fraction of alkali for each cementitious materials calculated by Eq. 5.20; and $\mathrm{mp}$ is the mass portion of alkali in chemical composition of each cementitous material (e.g. $0.08 \%$ $\mathrm{Na}_{2} \mathrm{O}$ in $\left.\mathrm{OPC}\right)$.

The pore solution volume (V) per $100 \mathrm{~g}$ of cementitious materials, depends only on initial liquid content including water or water plus superplasticizer if it is present in the mixture. As discussed in section 5.4.1, there is no bound water during the fresh state to be subtracted from initial water amount and it is assumed that no water loss occurs due to evaporation. Since the density of the liquid content was around $1.00 \mathrm{~g} / \mathrm{cm}^{3}$ at $25^{\circ} \mathrm{C}$, the volume of pore solution per $100 \mathrm{~g}$ of cementitious materials can be expressed as:

$V=(w / c \times 100)$ 
where $\mathrm{w}$ and $\mathrm{c}$ are the weight of liquid content (water plus superplasticizer) and entire cementitious materials in $\mathrm{g}$, respectively.

The concentration of $\mathrm{Na}^{+}$and $\mathrm{K}^{+}$was then calculated from Eq. 5.19 knowing $\mathrm{m}_{\mathrm{r}}$ from Eq. 5.21. Subsequently, $\mathrm{SO}_{4}{ }^{2-}$ and $\mathrm{OH}^{-}$concentrations were obtained using Eq. 5.1 and 5.2, respectively.

The total pore solution conductivity, $\sigma$, corresponding to above ions concentrations, can be obtained from Eq. 5.16 proposed for the pore solutions only filled with water with hydrodynamic viscosity, $\mu$, of $0.8999 \mathrm{mPa} . \mathrm{s}$ at $25{ }^{\circ} \mathrm{C}$ [12]. However, the viscosity enhancers in concrete such as superplasticizer increase the pore solution hydrodynamic viscosity which results in decrease in pore solution conductivity [60]. Therefore, Eq. 5.23 was used in our model to take into account the hydrodynamic viscosity of pore solution:

$\sigma_{c a l}=\left(\frac{\mu_{w}}{\mu_{p}}\right) \sum z_{i} C_{i} \lambda_{i}$

where $\mu_{\mathrm{w}}$ and $\mu_{\mathrm{p}}$ are hydrodynamic viscosities (mPa.s) of the water and pore solution (water plus superplasticizer) at $25^{\circ} \mathrm{C}$, respectively; and $\sigma_{\text {cal }}$ is the calculated pore solution conductivity based on the proposed model in this study. Therefore, if the liquid content of paste contains water plus superplasticizer, the weighted average of their hydrodynamic viscosities in the model can be calculated from

$$
\left(\frac{\mu_{p}}{\mu_{w}}\right)=\frac{S P}{w} \times \mu_{R}+\frac{W}{w}
$$


where w, W, and SP are the weights (g) of liquid content, water, and superplasticizer, respectively; and $\mu_{\mathrm{R}}$ is the relative hydrodynamic viscosity of the superplasticizer to that of water at $25^{\circ} \mathrm{C}$.

\subsubsection{Calibration of the model}

The basic Eq. 5.20, which was proposed to estimate the reacted fraction of each cementitious material, has two empirical constants $\mathrm{k}^{\prime}{ }_{1}$ and $\mathrm{k}_{2}^{\prime}$. These constants were calibrated for each cementitious material so that the predicted pore solution conductivity provides the best agreement with experimental results of the selected pastes. The five selected pastes had the closest value of w/c ratio (0.45) to practical application in construction industry. In addition, the medium dosage of each supplementary cementitious material and superplasticizer were chosen; i.e. $30 \%, 10 \%$, and $30 \%$ replacement in binder for fly ash, silica fume and slag, respectively, and $0.5 \%$ by OPC mass for superplasticizer were selected. The average conductivity data from three measurements at $25^{\circ} \mathrm{C}$ at each time bench mark (i.e., $30^{\text {th }}, 60^{\text {th }}$ and $90^{\text {th }}$ minute) were used for the calibration of the model as presented in Table 4.6. The total alkali $\left(\mathrm{Na}_{2} \mathrm{O}\right.$ and $\mathrm{K}_{2} \mathrm{O}$ ) contents as an input in the model for various cementitious materials were taken from chemical composition analysis presented in Table 5.5.

Table 5.5. Total $\mathrm{Na}_{2} \mathrm{O}$ and $\mathrm{K}_{2} \mathrm{O}$ contents in cementitious materials from chemical composition analysis.

\begin{tabular}{|c|c|c|c|c|}
\cline { 2 - 5 } \multicolumn{1}{c|}{} & $\begin{array}{c}\text { OPC } \\
(\%)\end{array}$ & $\begin{array}{c}\text { Silica Fume } \\
(\%)\end{array}$ & $\begin{array}{c}\text { Fly Ash } \\
(\%)\end{array}$ & $\begin{array}{c}\text { Slag } \\
(\%)\end{array}$ \\
\hline $\mathbf{N a}_{2} \mathbf{O}$ & 0.08 & 0.08 & 0.94 & 0.30 \\
$\mathbf{K}_{2} \mathbf{O}$ & 0.56 & 0.92 & 1.78 & 0.42 \\
\hline
\end{tabular}


The experimental results in Table 4.6 are plotted in Fig. 4.8, which was used for the calibration of the model. Various findings can be observed from this figure. It shows that after 30 minutes from mixing, the increase in conductivity is smooth and almost linear. Also, all SCMs reduced the pore solution conductivity which can be attributed to their delayed reactions with water compared to OPC. The most significant reduction occurred when the paste was incorporated with slag. On the other hand, superplasticizer addition which increases the viscosity of pore solution, resulted in less conductivity values which were in agreement with literature [60]. Additionally, the rate of increase in conductivity was the same order for all types of pastes, except the one with silica fume whose rate was around twice of others.

From the pure analytical point of view, constant $\mathrm{k}_{2}^{\prime}$ in Eq. 5.20 is more indicative of magnitude of $\mathrm{F}$, whereas constant $\mathrm{k}_{1}^{\prime}$ mainly represents the rate of change in $\mathrm{F}$. They are both directly proportional to F; i.e., the greater the constants, the higher the resultant F.

Using data demonstrated in Fig. 4.8, the proposed model was calibrated by selecting appropriate values for $\mathrm{k}_{1}^{\prime}$ and $\mathrm{k}_{2}{ }_{2}$ (Table 5.6) in a way that the best agreement between the predicted results and the experimental ones obtained as shown in Fig. 5.1. It is noted that in our experiments when OPC was incorporated with slag especially in high dosage, slag retarded the rate of OPC reaction. This effect was taken into account using a modified equation for $\mathrm{k}_{2}^{\prime}$ for OPC as presented in Table 5.6. 
Table 5.6. Empirical constants obtained from calibration for OPC, fly ash, silica fume and slag in Eq. 5.20.

\begin{tabular}{|c|cccc|}
\cline { 2 - 5 } \multicolumn{1}{c|}{} & OPC & SF & FA & SL \\
\hline $\mathbf{k}^{\prime}{ }_{1}$ & 0.170 & 0.290 & 0.160 & 0.140 \\
$\mathbf{k}^{\prime}{ }_{\mathbf{2}}$ & $0.76-\left[\exp \left(0.0002 \times R_{S L}\right)^{2.2}-1\right]$ & 0.150 & 0.026 & 0.0015 \\
\hline
\end{tabular}

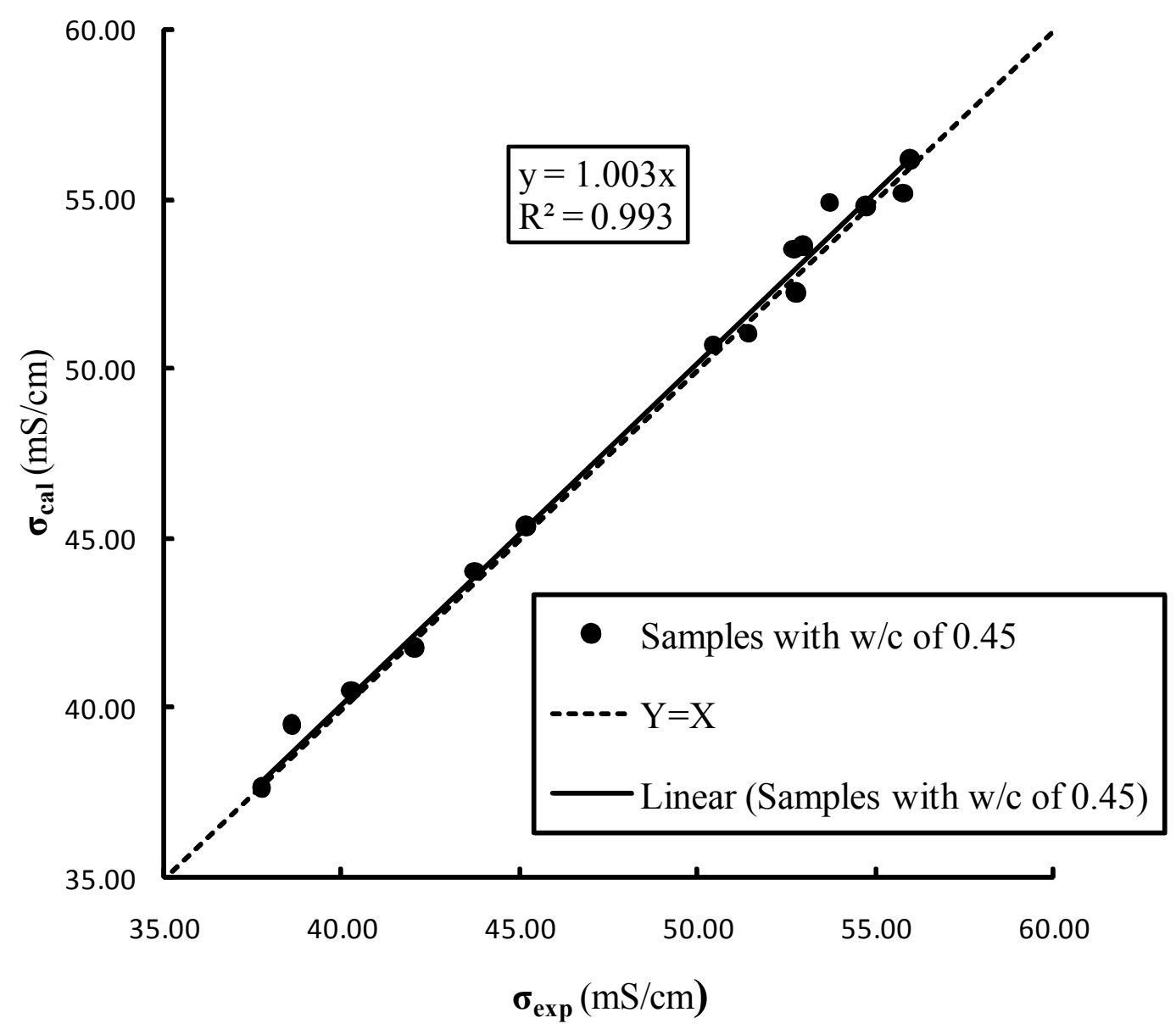

Figure 5.1. Calculated and experimental conductivity relationship after calibration of the proposed model for w/c of 0.45 . 


\subsection{Experimental Validation}

The validity of the proposed model was tested for all 91 pore solution samples used in this study. The pore solution conductivity calculated from the model, $\sigma_{\text {cal }}$, were plotted versus those from our experimental data, $\sigma_{\text {exp }}$, in Fig. 5.2 to evaluate their agreement. The results showed that the model captured the experimental results data quite well; i.e., the linear trend line was almost the same as $45^{\circ}$ line. The comparison between the estimated data and the experimental ones also indicated a strong correlation between these two data; i.e., $\mathrm{R}^{2}=0.89$. The same results marked separately for each type of the paste mixture used in this study are shown in Fig. 5.3. As seen, for very high dosage of slag (i.e., 50\%) which is not a practical usage, the difference between the estimated and the experimental data are more significant compared to the rest of data in Fig. 5.3. Most probably the dissolution and hydration mechanisms and subsequently the concentration of ions in pore solution are significantly different at very high dosages of slag. Therefore, the accuracy of the proposed model for very high dosages of slag would be compromised. 


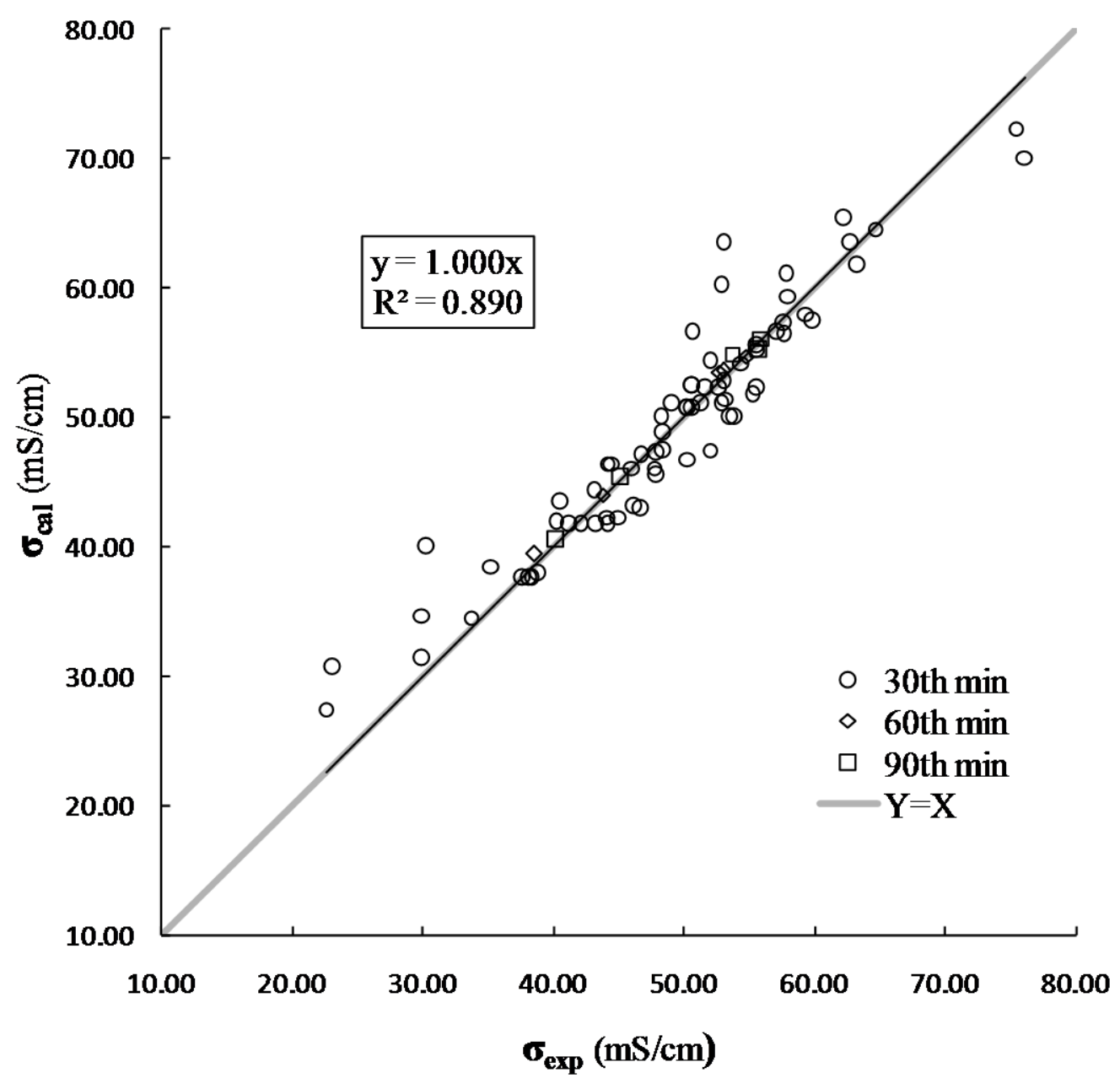

Figure 5.2. Calculated pore solution conductivity from the proposed model versus those from experimental data. 


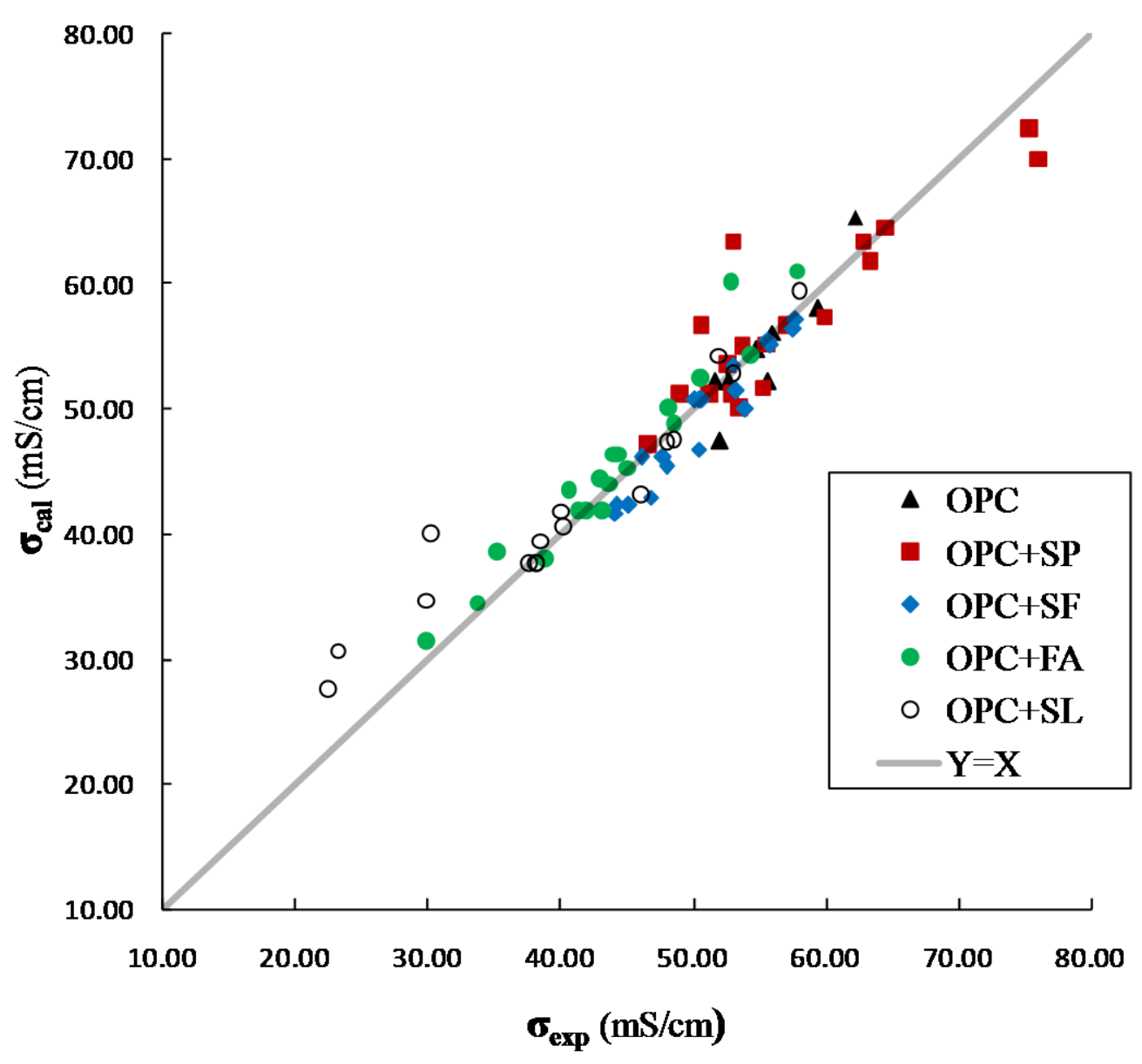

Figure 5.3. Calculated pore solution conductivity from the proposed model versus those from the experimental data.

\subsection{Discussion}

The proposed model can successfully estimate the pore solution conductivity of various mixtures used in this study. It takes into account wide range of possible scenarios that exist for a paste mixture and predicts the time dependency of pore solution conductivity up to the initial setting time which is onset of solidification due to hydration products 
formation. Further studies also need to be conducted to include the effect of other types of cementitious materials and admixtures on the accuracy of the model.

The effect of w/c ratio which can vary from 0.35 to 0.55 is considered in the model. Since the less portion of liquid content (w) compared to total cementitious materials/binder (c), corresponds to lower amount of ions released per unit volume of pore solution, the resulting concentration of ions decreases. Consequently, the conductivity of pore solution decreases. This effect was considered in the model through the volume of pore solution per $100 \mathrm{~g}$ of binders in Eq. 5.22.

Furthermore, the types of materials used in the mixtures categorized into five paste mixtures: OPC, OPC plus superplasticizer, OPC plus fly ash, OPC plus silica fume, and OPC plus slag. For each w/c ratio, at any particular time during fresh state, the control paste containing OPC and distilled water showed the highest conductivity which was attributed to faster reactions of cement particles compared to supplementary cementitious materials. Silica fume incorporated paste had the lowest drop in conductivity, while the drop in conductivity for slag and fly ash was quite significant, which exhibited more delayed pozzolanic reactions. As a result, less released ions in the pore solution that corresponds to less conductivity. For the pastes incorporated with $50 \%$ of slag in binder, the experimental values dropped significantly so that the calculated conductivities from the model were overestimated. This high usage of slag is not practical in concrete industry and could even delay the dissolution of cement particles in the pore solution which results in very low concentration of ions and thus quite small conductivity. However, this effect is formulized in constant $k_{2}$ of OPC. It is a function of $\mathrm{R}_{\mathrm{SL}}$ to take 
into account the retarding effect of slag on cement particles reactions (Table 5.6). The presence of superplasticizer in the pore solution was observed to have a small reduction effect on conductivity; e.g. from 54.76 to 52.72 at $60^{\text {th }}$ min. In the model, it was assumed that this small drop is attributed to increase in hydrodynamic viscosity of liquid part which has been shown to have reduction effect on the conductivity [60]. However, this observation may be also related to the release of water molecules trapped between cement particles by superplasticizer. The contribution of additional water molecules in pore solution can decrease the concentration of ions. It is also assumed that superplasticizer has no effect on the amount of released ions from cementitious materials. The experimental and calculated conductivity of superplasticizer incorporated pastes exhibited good agreement as shown in Fig. 5.3.

In addition, for the $w / c$ of 0.45 , the conductivity development with time during fresh state from $30^{\text {th }}$ to $90^{\text {th }}$ min suggested that rate of increase in pore solution conductivity for all types of pastes are in the same order, except silica fume whose rate is considerably higher (Fig. 4.8). This increase in conductivity is attributed to further reaction of cementitious materials particles with water. As shown in Fig. 5.4, the proposed model also exhibited that right after the mixing of materials corresponding to zero in time, pore solution conductivity has a quite sharp increase during the first few minutes, whereas the rate of the conductivity increase after the first few minutes drops significantly and thus pore solution conductivity smoothly increases over time until initial setting time. 


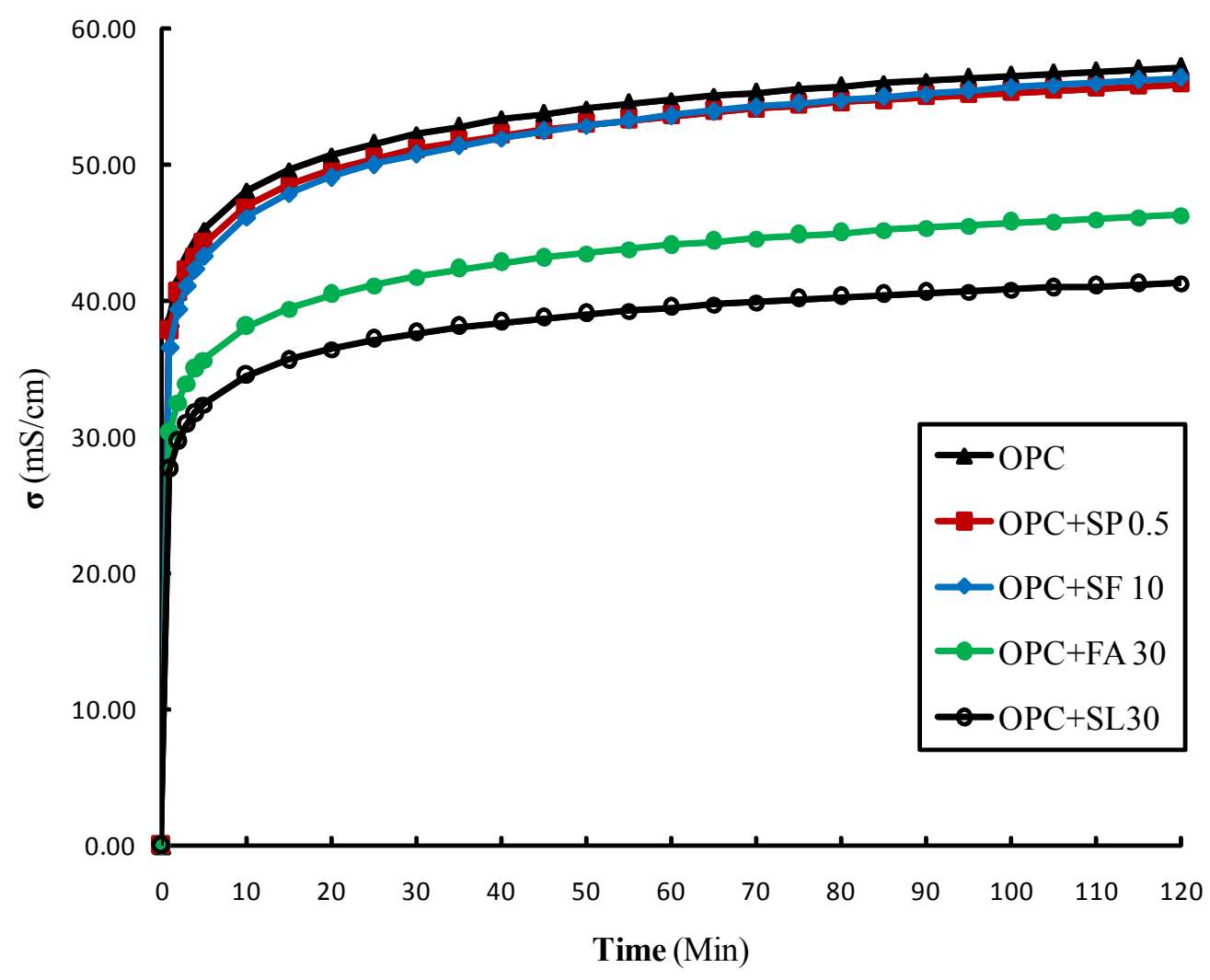

Figure 5.4. Pore solution conductivity development over time calculated by the proposed model for paste mixtures with w/c of 0.45 during first 2 hours. 


\section{Conclusion and Future works}

\subsection{Conclusions}

The main conclusions from this research are highlighted as follows:

- Pore solution conductivity increases with time which corresponds to reduction in resistivity at fresh state of cement paste. As time proceeds, progress in chemical reactions of cementitious materials with water results in further dissolution of ions in pore solution; hence, the ions concentration in pore solution increases which are the source of electrical charge transfer and consequently the increase in pore solution conductivity.

- By increasing the $\mathrm{w} / \mathrm{c}$ of the pastes the conductivity of the pore solution at fresh state (i.e., 2 hours after mixing before the setting time) decreases, which is attributed to the less ions concentration released in pore solution. The decrease of ions concentration in pore solution results in decrease in conductivity which corresponds to increase in resistivity.

- SCMs and superplasticizer reduce the corresponding pore solution conductivity at 30 minutes after mixing during fresh state when added to the OPC paste. Superplasticizer lowers the pore solution conductivity by increasing the dynamic viscosity of pore solution which results in less electrical charge transfer in pore solution. SCMs delay the dissolution of released ions in pore solution because of retarding effect on chemical reactions between solid particles and liquid phase and consequently the pore solution conductivity decreases. The magnitude of the drop in 
pore solution conductivity from the highest to the lowest is related to slag, fly ash, silica fume and superplasticizer, respectively. However, at later times during fresh state (e.g. longer than $90^{\text {th }}$ minute), the higher rate of conductivity rise in silica fume (mS/cm.min) results in pore solution conductivity values close to that of the OPC. This considerable higher rate of conductivity development in silica fume is most likely attributed to it surface area $\left(\mathrm{cm}^{2} / \mathrm{g}\right)$ which is several times higher than that of the OPC, fly ash or slag.

- Paste electrical resistivity decreases with time along with that of pore solution while their ratio, formation factor $\mathrm{F}$, is almost constant during fresh state. The resistivity of the paste as a porous media is a function of pore solution resistivity (chemical effect) as well as w/c and solid microstructure (physical effect). Higher pore solution resistivity and lower $\mathrm{w} / \mathrm{c}$ of paste correspond to higher paste resistivity. The formation factor only exhibits the physical effect and thus is affected by porosity and solid microstructure (e.g. particle size and distribution in the case of fresh cement paste). Therefore, the increase in paste electrical resistivity at later times is only attributed to increase in pore solution resistivity and the steadiness of formation factor over time at fresh state indicates the absence of solidification or microstructure change.

- At fresh state, although the paste electrical resistivity shows a small drop by increasing $\mathrm{w} / \mathrm{c}$ for low and medium range of $\mathrm{w} / \mathrm{c}(\mathrm{i} . \mathrm{e} ., \quad<0.5)$, it rises again at very high w/c values (i.e., $>0.5$ ). Therefore, there is no constant descending order like the one was observed in the hardened state. This variation is attributed to opposing effect of 
physical and chemical influential factors when the w/c increases; i.e., if w/c increases for a paste, the porosity which represents volumetric fraction of liquid content in paste matrix increases which results in drop in paste resistivity, whereas pore solution electrical resistivity increases which corresponds to a rise in the paste resistivity. As a result, when physical effect governs paste resistivity decreases; however. dominating the chemical effect (in very high $\mathrm{w} / \mathrm{c}$ ratios) results in increase in paste electrical resistivity.

- The formation factor $\mathrm{F}$ in a cement paste reveals a promising descending order with respect to $\mathrm{w} / \mathrm{c}$ or porosity; i.e., the greater the $\mathrm{w} / \mathrm{c}$ and subsequently porosity, the lower the corresponding formation factor. The F-w/c or F- $\varphi$ relationship can be strongly correlated through Archie's law. So, using the proposed numerical model for pore solution conductivity as well as measuring the paste resistivity, w/c ratio can be estimated through the experimental correlation presented in this research.

- The tortuosity which indicates the geometric complexity of electrical charge movement in the pore solution due to solid particles size and their distribution affects the magnitude of the formation factor. For a same porosity value, the larger particle size and the rounder particle shape as well as higher cement particle aggregation correspond to lower tortuosity. Accordingly, the tortuosity increases in paste mixtures with superplasticizer, whereas slag and fly ash incorporated pastes decrease the tortuosity of the cement paste system. Silica fume has almost the same tortuosity as OPC. 
- $\mathrm{pH}$ of the cement paste exhibits a strong correlation with pore solution conductivity; i.e., similar to conductivity it increases over time and decreases with increase in the $\mathrm{w} / \mathrm{c}$ of the pastes. Therefore, the $\mathrm{pH}$ measurement provides a faster, easier and accurate alternative to estimate the pore solution conductivity of concrete.

- Based on the numerical model proposed in this study, the development of pore solution conductivity with time was characterized. It follows an exponential trend; i.e., within very first few minutes after mixing the materials, the pore solution conductivity increases with a sharp slope followed by a smooth increase (almost linear) until setting initiates .

\subsection{Recommendations for Future Work}

Following is the list of some research areas related to the course of this study that requires further investigation:

- The experimental tests of this study were conducted on the most significant constituent of concrete, cement paste. However, the presence of aggregates in concrete and mortar affects their tortuosity and hence their electrical resistivity. Aggregates can also affect pore solution conductivity if they are contaminated. Thus, the effect of aggregates needs further systematic studies.

- The cement used in this study was Ordinary Portland Cement (i.e., Type I or II) which is generally used in all pertinent construction projects. But, the electrical resistivity characteristics of other types of cements (e.g. Type III or Type V) also need to be investigated. 
- In this research, it was assumed that volume of the pores between cementitous materials is fully filled with water. However, the presence of air voids (i.e., air content) can also affect the resistivity of cement paste which requires further studies.

- Only the effect of superplasticizer as a chemical admixture was included in this study. This can be further extended to other types of admixtures such as accelerators, retarders, corrosion inhibitors or air-entraining admixtures.

- The numerical model proposed in this study can estimate the pore solution conductivity only in the fresh state and before setting and hardening. It can be further developed for hardened state to take into account the effect of bound water and taken up of alkali cations by hydration products. 


\section{References}

1. Monfore, G.E., The electrical resistivity of concrete. Journal of the PCA Research and Development Laboratories, 1968. 10(2): p. 35-48.

2. Wei, X., Early age compressive strength of pastes by electrical resistivity method and maturity method. Journal of Wuhan University of Technology-Mater. Sci. Ed., 2011. 26(5): p. 983-989.

3. Hammond, E. and T. Robson, Comparison of electrical properties of various cements and concretes. The Engineer, 1955. 199(5156): p. 114.

4. Li, Z. and W. Li, No-Contacting Method for Resistivity Measurement of Concrete Specimen, in U.S. and China Patent. 2001.

5. Li, Z., X. Wei, and W. Li, Preliminary interpretation of Portland cement hydration process using resistivity measurements. ACI Materials Journal, 2003. 100(3).

6. Li, Z., L. Xiao, and X. Wei, Determination of concrete setting time using electrical resistivity measurement. Journal of materials in civil engineering, 2007. 19(5): p. 423-427.

7. Liu, Y. and F.J. Presuel-Moreno, Normalization of Temperature Effect on Concrete Resistivity by Method Using Arrhenius Law (with Appendix). ACI Materials Journal, 2014. 111(1-6).

8. Mancio, M., et al., Instantaneous Determination of Water-Cement Ratio of Fresh Concrete. ACI Materials Journal, 2010. 107(6).

9. Salem, T.M., Electrical conductivity and rheological properties of ordinary Portland cement-silica fume and calcium hydroxide-silica fume pastes. Cement and concrete research, 2002. 32(9): p. 1473-1481. 
10. Salem, T.M. and S.M. Ragai, Electrical conductivity of granulated slag-cement kiln dust-silica fume pastes at different porosities. Cement and concrete research, 2001. 31(5): p. 781-787.

11. Sant, G., et al. Electrical conductivity measurements in cement paste at early ages: a discussion of the contribution of pore solution conductivity, volume, and connectivity to the overall electrical response. in -Int. RILEM Workshop on Advanced Testing of Fresh Cementitious Materials. 2006.

12. Snyder, K., et al., Estimating the electrical conductivity of cement paste pore solutions from $\mathrm{OH}^{-}, \mathrm{K}+$ and $\mathrm{Na}+$ concentrations. Cement and Concrete Research, 2003. 33(6): p. 793-798.

13. Topçu, İ.B., T. Uygunoğlu, and İ. Hocaoğlu, Electrical conductivity of setting cement paste with different mineral admixtures. Construction and Building Materials, 2012. 28(1): p. 414-420.

14. Wei, X. and Z. Li, Early hydration process of Portland cement paste by electrical measurement. Journal of materials in civil engineering, 2006. 18(1): p. 99-105.

15. Xiao, L. and Z. Li, Early-age hydration of fresh concrete monitored by noncontact electrical resistivity measurement. Cement and Concrete Research, 2008. 38(3): p. 312-319.

16. Whittington, H., J. McCarter, and M. Forde, The conduction of electricity through concrete. Magazine of concrete research, 1981. 33(114): p. 48-60.

17. McCarter, W. and S. Garvin, Dependence of electrical impedance of cementbased materials on their moisture condition. Journal of Physics D: Applied Physics, 1989. 22(11): p. 1773.

18. Saad, M., et al., Effect of silica fume on the phase composition and microstructure of thermally treated concrete. Cement and concrete research, 1996. 26(10): p. 1479-1484. 
19. Archie, G.E., The electrical resistivity log as an aid in determining some reservoir characteristics. Transactions of the American Institute of Mining and Metallurgical Engineers 1942. 146: p. 54-62.

20. ASTM, Standard test method for time of setting of concrete mixtures by penetration resistance, in C. 2005, American Society of Testing and Materials.

21. Michelsen, S., Beitrag zur bindezeit bsestimmung. Zement, 1933. 22(3): p. 457461.

22. Gu, P., et al., Investigation of the retarding effect of superplasticizers on cement hydration by impedance spectroscopy and other methods. Cement and concrete research, 1994. 24(3): p. 433-442.

23. Torrents, J., J. Roncero, and R. Gettu, Utilization of impedance spectroscopy for studying the retarding effect of a superplasticizer on the setting of cement. Cement and Concrete Research, 1998. 28(9): p. 1325-1333.

24. McCarter, W., et al., Characterization and monitoring of cement-based systems using intrinsic electrical property measurements. Cement and Concrete Research, 2003. 33(2): p. 197-206.

25. Xiao, L.-Z., Z.-j. Li, and X.-s. Wei, Selection of superplasticizer in concrete mix design by measuring the early electrical resistivities of pastes. Cement and Concrete Composites, 2007. 29(5): p. 350-356.

26. Zong-jin, L. and W. Xiao-sheng, The electrical resistivity of cement paste incorporated with retarder. Journal of Wuhan University of Technology-Mater. Sci. Ed., 2003. 18(3): p. 76-78.

27. Wei, X. and L. Xiao, Influence of the aggregate volume on the electrical resistivity and properties of portland cement concretes. Journal of Wuhan University of Technology-Mater. Sci. Ed., 2011. 26(5): p. 965-971. 
28. Hansson, L.H. and C.M. Hansson, Electrical Resistivity Measurements of Portland Cement-Based Materials. Cement and Concrete Research, 1983. 13(5): p. 675-683.

29. Maguire, D. and M. Olen, Report on an investigation into the electrical properties of concrete. Transactions of the South African Institute of Electrical Engineers, 1940. 31: p. 301.

30. Nikkannen, P., On electrical properties of concrete and their application. Valtion Teknillinen Tutkimuslaitos. Tiedotus. Saraj 1962. III(60): p. 175.

31. Hughes, B., A. Soleit, and R. Brierley, New technique for determining the electrical resistivity of concrete. Magazine of concrete research, 1985. 37(133): p. 243-248.

32. Terry, E.M., Advanced Laboratory Practice in electricity and magnetism. Second ed. 1929, N.Y.: McGraw-Hill. 197.

33. Powers, T.C., Studies of the physical properties of hardened Portland cement paste. 1948, Chicago: [Portland Cement Association, Research Laboratories].

34. Taylor, H.F.W., Cement chemistry. 2003, London: Telford Publ.

35. Taylor, H.F., K. Mohan, and G. Moir, Analytical Study of Pure and Extended Portland Cement Pastes: II, Fly Ash-and Slag-Cement Pastes. Journal of the American Ceramic Society, 1985. 68(12): p. 685-690.

36. Longuet, P., L. Burglen, and A. Zelwer, The liquid phase of hydrated cement. des Materiaux de Construction et de Travaux Pubics, 1973. 676: p. 35-41.

37. Taylor, H.F., A method for predicting alkazi ion concentrations in cement pore solutions. Advances in Cement Research, 1987. 1(1): p. 5-17.

38. Reardon, E.J., Problems and approaches to the prediction of the chemical composition in cement/water systems. Waste management, 1992. 12(2): p. 221239. 
39. Horvath, A.L., Handbook of aqueous electrolyte solutions : physical properties, estimation, and correlation methods. 1985, Chichester; New York: Ellis Horwood ; Halsted Press.

40. Bertolini, L., R. Polder, and T.N.O. Building, Concrete resistivity and reinforcement corrosion rate as a function of temperature and humidity of the environment. 1997, Delft, The Netherlands: Netherlands Organisation for Applied Scientific Research.

41. Villagrán Zaccardi, Y., et al., Influence of temperature and humidity on Portland cement mortar resistivity monitored with inner sensors. Materials and corrosion, 2009. 60(4): p. 294-299.

42. Chrisp, T., et al., Temperature-conductivity relationships for concrete: An activation energy approach. Journal of materials science letters, 2001. 20(12): p. 1085-1087.

43. McCarter, W., et al., Field monitoring of electrical conductivity of cover-zone concrete. Cement and Concrete Composites, 2005. 27(7): p. 809-817.

44. Watanabe, H., MEASUREMENTS OF ELECTRICAL CONDUCTIVITY OF BASALT AT TEMPERATURES UP TO $1500^{\circ} \mathrm{C}$ AND PRESSURES TO ABOUT 20 KILOBARS. Special Contributions of the Geophysical Institute, Kyoto University, 1970. 10: p. 159-170.

45. Arrhenius, S., On the theory of chemical reaction velocity. Zeitschrift für Physikalische Chemie, 1899. 28: p. 317.

46. Castellote, M., C. Andrade, and M.C. Alonso, Standardization, to a Reference of 25? C, of Electrical Resistivity for Mortars and Concretes in Saturated or Isolated Conditions. ACI Materials Journal, 2002. 99(2): p. 119-128.

47. McCarter, W., G. Starrs, and T. Chrisp, Electrical conductivity, diffusion, and permeability of Portland cement-based mortars. Cement and Concrete Research, 2000. 30(9): p. 1395-1400. 
48. Pour-Ghaz, M., O.B. Isgor, and P. Ghods, The effect of temperature on the corrosion of steel in concrete. Part 1: Simulated polarization resistance tests and model development. Corrosion Science, 2009. 51(2): p. 415-425.

49. Bockris, J.O.M., A.K.N. Reddy, and M.E. Gamboa-Aldeco, Modern electrochemistry. 1998, New York: Plenum Press.

50. Elkey, W. and E.J. Sellevold, Electrical resistivity of concrete. Norwegian Public Roads Administration Publication, 1995: p. 33.

51. Morsy, M.S., Effect of temperature on electrical conductivity of blended cement pastes. Cement and concrete research, 1999. 29(4): p. 603-606.

52. McCARTER, W. and A. Afshar. A METHOD FOR QUANTIFYING THE EFFECT OF ADMIXTURES ON THE SETTING OF CEMENT. TECHNICAL NOTE. in ICE Proceedings. 1987. Thomas Telford.

53. Maxwell, J.C., Treatise on electricity and magnetism. 1, 1. 1998, Oxford: Oxford University Press.

54. Slawinski, A., Conductivity of an Electrolyte Containing Dielectric Bodies. Jour. Chem. Phys, 1926. 23: p. 710-727.

55. Fricke, H., The electric conductivity and capacity of disperse systems. Journal of Applied Physics, 1931. 1(2): p. 106-115.

56. Pirson, S.J., Electric logging factors which affect true formation resistivities Oil and Gas Journal 1947. 46(26): p. 76-81.

57. Atkins Jr, E. and G. Smith, The significance of particle shape in formation resistivity factor-porosity relationships. Journal of Petroleum Technology, 1961. 13(03): p. 285-291.

58. Buenfeld, N. and J. Newman, Examination of three methods for studying ion diffusion in cement pastes, mortars and concrete. Materials and Structures, 1987. 20(1): p. 3-10. 
59. http://www.giatecscientific.com/product/giatec-rcon/english-rcon2/. [cited 2015 July 21].

60. Bentz, D.P., et al., VERDiCT: viscosity enhancers reducing diffusion in concrete technology. Concrete International, 2009. 31(1): p. 31-36.

61. Bentz, D.P., K.A. Snyder, and A. Ahmed, Anticipating the Setting Time of HighVolume Fly Ash Concretes Using Electrical Measurements: Feasibility Studies Using Pastes. Journal of Materials in Civil Engineering, 2014.

62. Glasser, F.P. and J. Marr, Alkali binding potential of OPC and blended cements. Cemento, 1985. 82(2): p. 85-94.

63. Struble, L.J., The influence of cement pore solution on alkali-silica reaction. 1987, Purdue University.

64. Dalziel, J. and W. Gutteridge, The influence of pulverized-fuel ash upon the hydration characteristics and certain physical properties of a Portland cement paste. 1986.

65. Taylor, H.F. Bound water in cement pastes and its significance for pore solution compositions. in MRS Proceedings. 1986. Cambridge Univ Press.

66. Harrisson, A., H.F. Taylor, and N. Winter, Electron-optical analyses of the phases in a Portland cement clinker, with some observations on the calculation of quantitative phase composition. Cement and Concrete Research, 1985. 15(5): p. 775-780.

67. Bhatty, M. and N. Greening. Interaction of alkalies with hydrating and hydrated calcium silicates. in Proceedings. 1978.

68. Coole, M. Calorimetric studies of the hydration behaviour of extended cements. in Proc. Br. Ceram. Soc. 1984. 


\section{Appendix A: Basic Definitions}

\section{A.1. Pore solution}

The cement paste matrix consists of two components; (1) solid particles or cementitious materials; (2) liquid component which mainly includes water and can contain minor amount of liquid chemical admixture such as superplasticizer. Since all the pores between solid particles are filled with liquid component, latter is referred as pore solution in this thesis.

\section{A.2. Porosity in the paste}

Porosity in a porous material such as rocks or soils is defined as the volumetric ratio of air voids to that of total material (i.e., solid, air and liquid). In a fresh cement paste, all the air voids (pores) are almost filled with water and saturation degree is $100 \%$. Therefore, the volume of water is equal to that of air voids and porosity $(\varphi)$ in a cement paste is defined as the volumetric ratio of water content to that of water plus cemetitious materials. In Fig. A.1, the porosity is the ratio of the white area to shaded area plus white area.

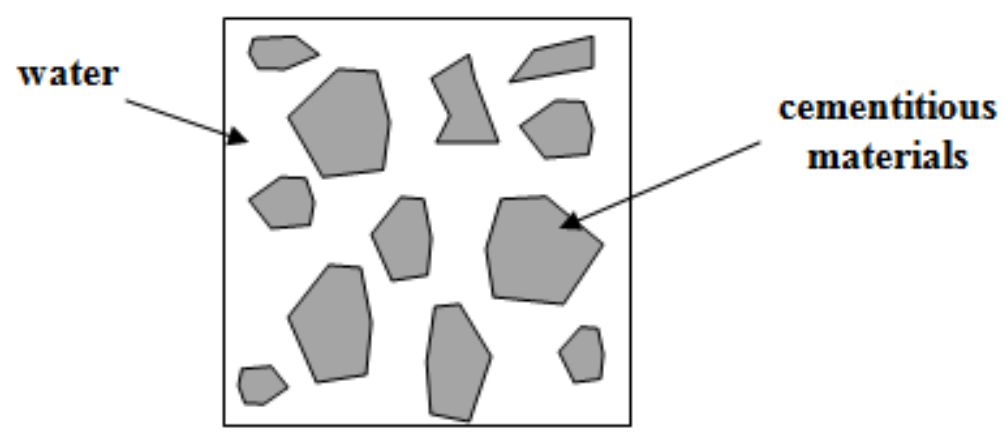

Figure A.1. Schematic representation of cement paste structure in fresh state. 


\section{A.3. Chemical and physical effect on paste resistivity}

The paste electrical resistivity is affected by pore solution electrical resistivity, porosity, and solid particles size and distribution in the liquid (water). Pore solution electrical resistivity/conductivity is resulted from ions dissolved into water as a result of chemical reaction of cementitious materials and water whereas porosity and solid particles size and distribution in water only represents the physical effect of cementitious materials in the paste. Accordingly, the former and latter are called chemical and physical effect, respectively.

\section{A.4. Formation factor $F$}

The formation factor was first proposed by Archie [19] for rocks saturated $100 \%$ with water. It was defined as the ratio of rocks electrical resistivity to water electrical resistivity contained in them. However, the formation factor of cement paste in this research is defined as the ratio of paste electrical resistivity to corresponding pore solution electrical resistivity. The details are discussed in Chapter 2 of this thesis.

\section{A.5. Tortuosity}

The electrical resistivity is a material property which quantifies the ease of electrical charge transfer through the material; i.e., the easier movement of ions through the material results in the lower corresponding electrical resistivity. In a cement paste as a two-component system (i.e., solid particles and pore solution), the pore solution is the conductive component. The electrical transport occurs by the movement of ions in the cement pore solution among the solid particles. The solid particles act as barrier against 
the charge transport and increase the resistivity. However, for a given porosity or solid particles content, electrical resistivity is also affected by the size, shape, and distribution of the solid particles. This factor is quantified as an experimental constant called tortuosity. Therefore, tortuosity in a cement paste indicates geometric complexity or the extent of convoluted paths for the movement of ions through solid particles. Fig. A.2 shows how the roundness, the size (i.e., higher number of particles for certain porosity) as well as distribution of solid particles affects the tortuosity. 


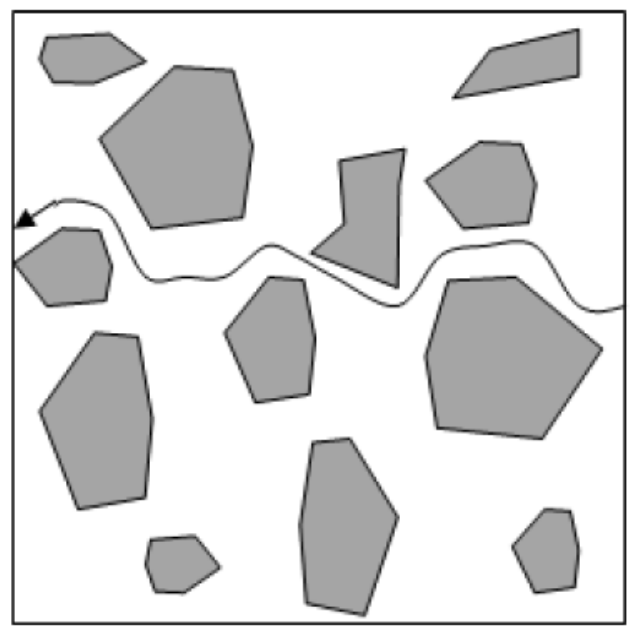

(a)

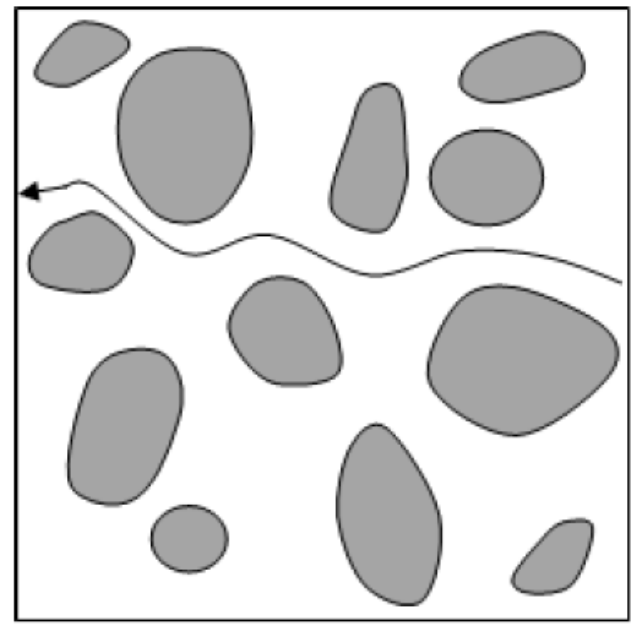

(c)

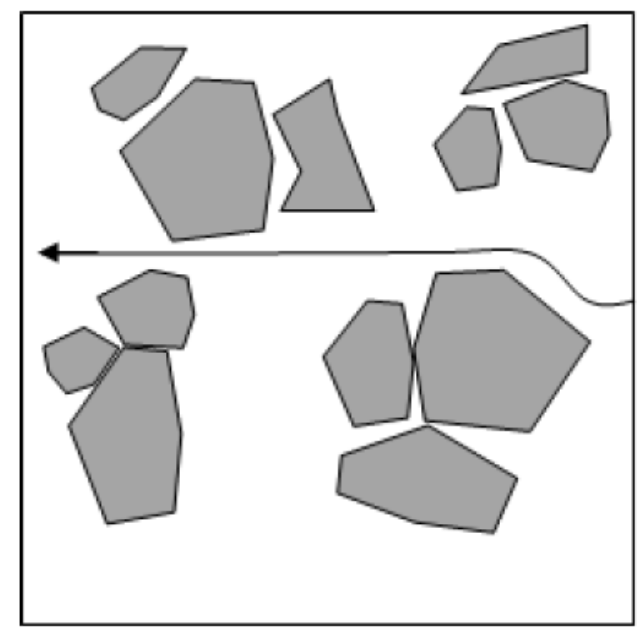

(b)

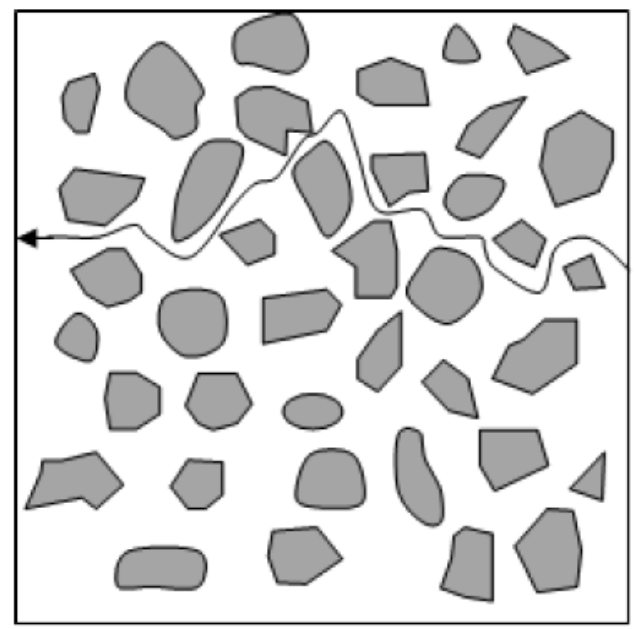

(d)

Figure A.2. Schematic of the ions transport in the pore solution among the solid particles with the same porosity: a) normal distribution of particles; b) aggregated particles; c) round shape particles; and d) small size particles. 


\section{Appendix B: Supplementary Figures for Chapter 4}

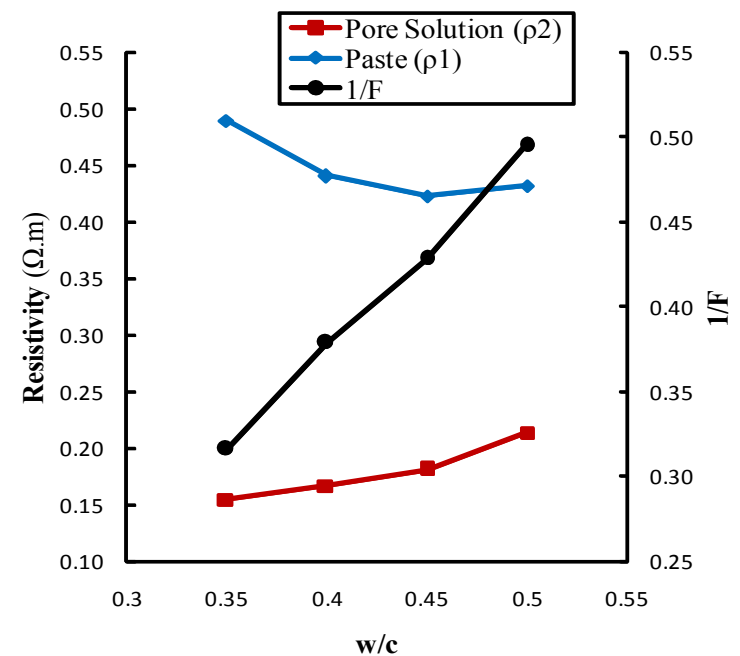

(a)

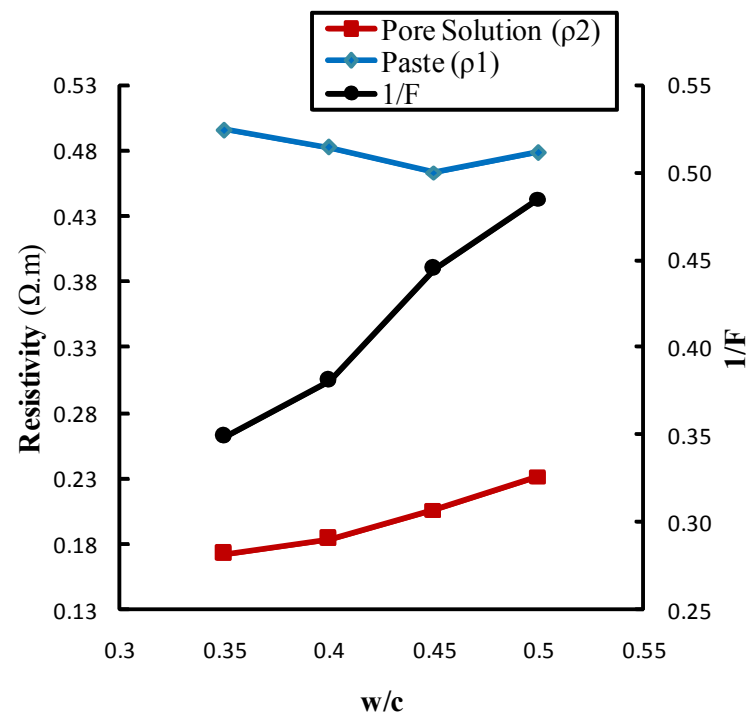

(c)

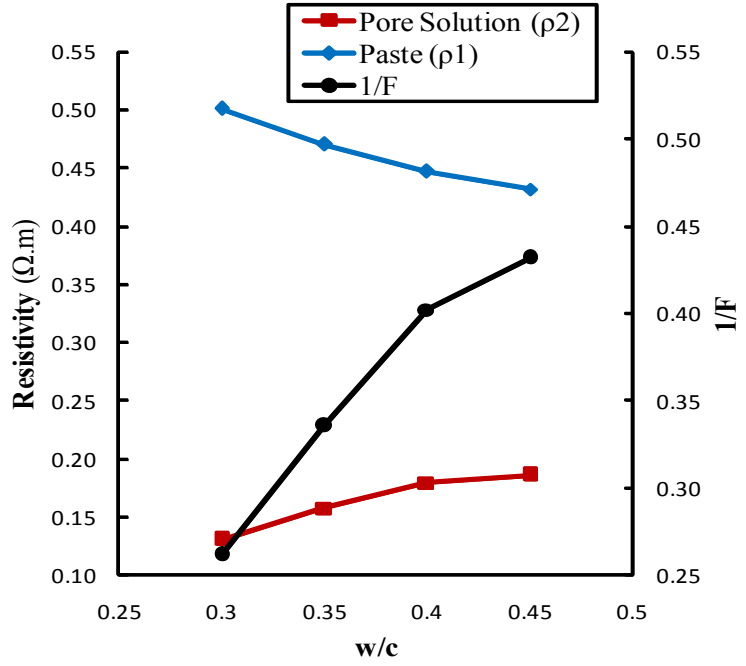

(b)

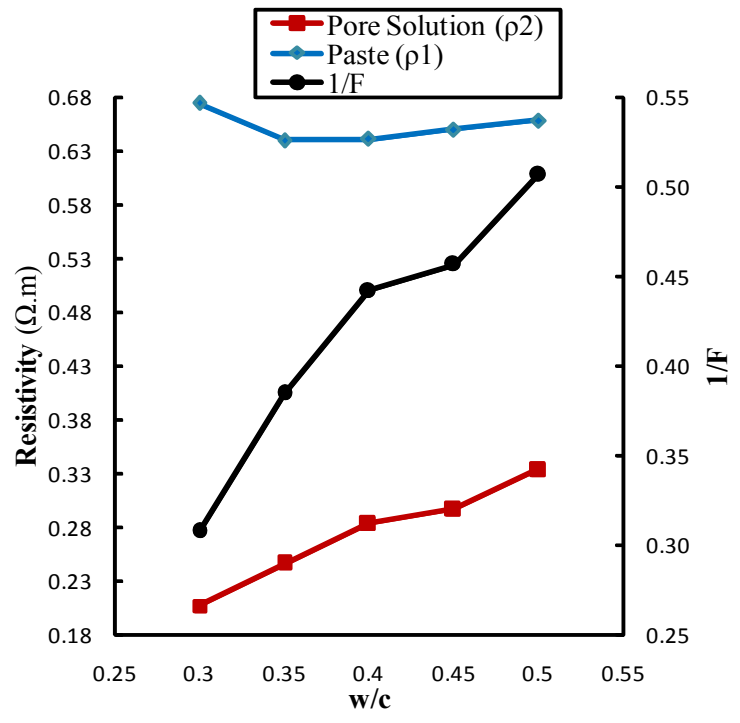

(d)

Figure B.1. Paste and pore solution resistivity as well as inverse of formation factor versus $\mathrm{w} / \mathrm{c}$ at $30^{\text {th }}$ minute of paste age: a) OPC plus $0.2 \%$ superplasticizer; b) OPC plus 1.0\% superplasticizer; c) OPC plus 10\% fly ash; d) OPC plus 50\% fly ash; e) OPC plus $5 \%$ silica fume; f) OPC plus $15 \%$ silica fume; g) OPC plus $10 \%$ slag; h) OPC plus 50\% slag 


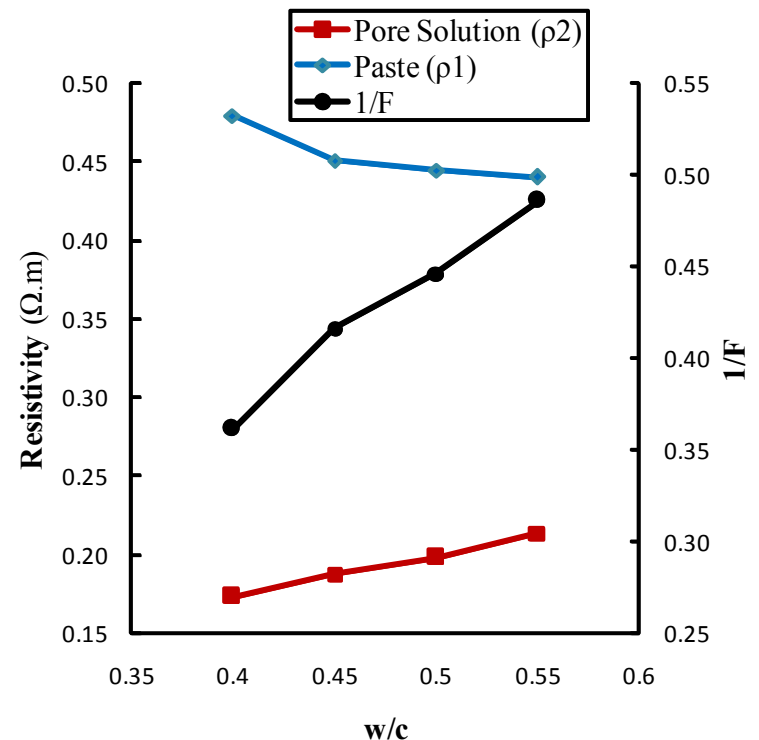

(e)

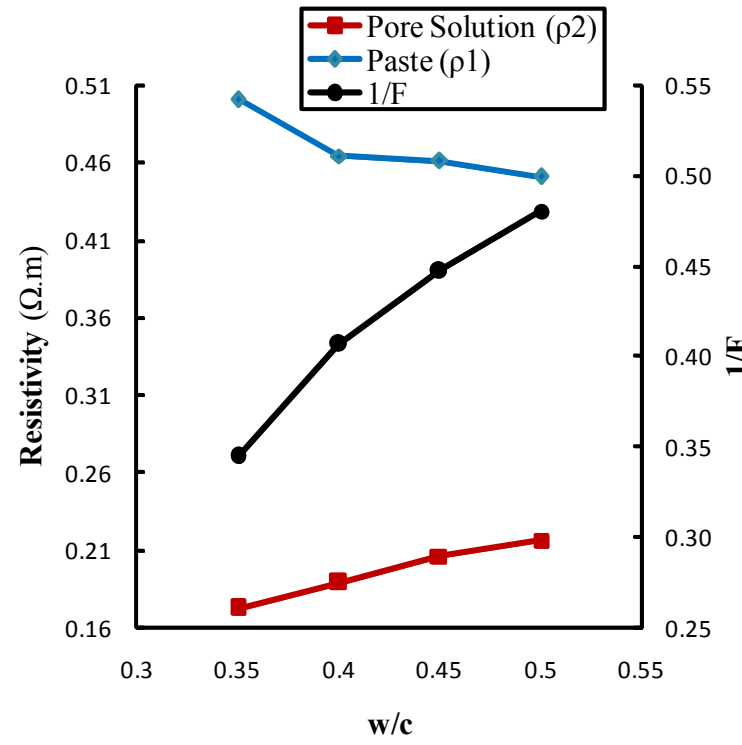

(g)

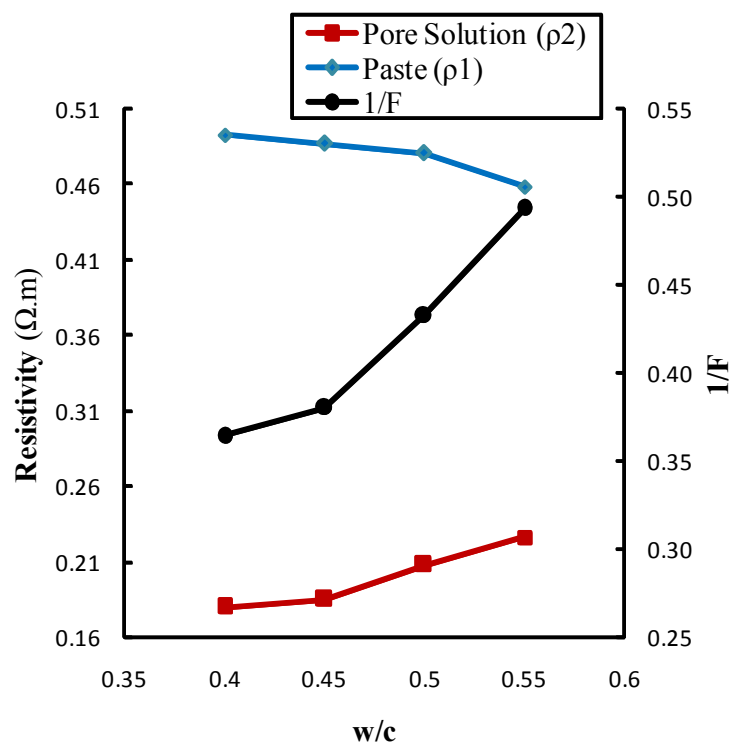

(f)

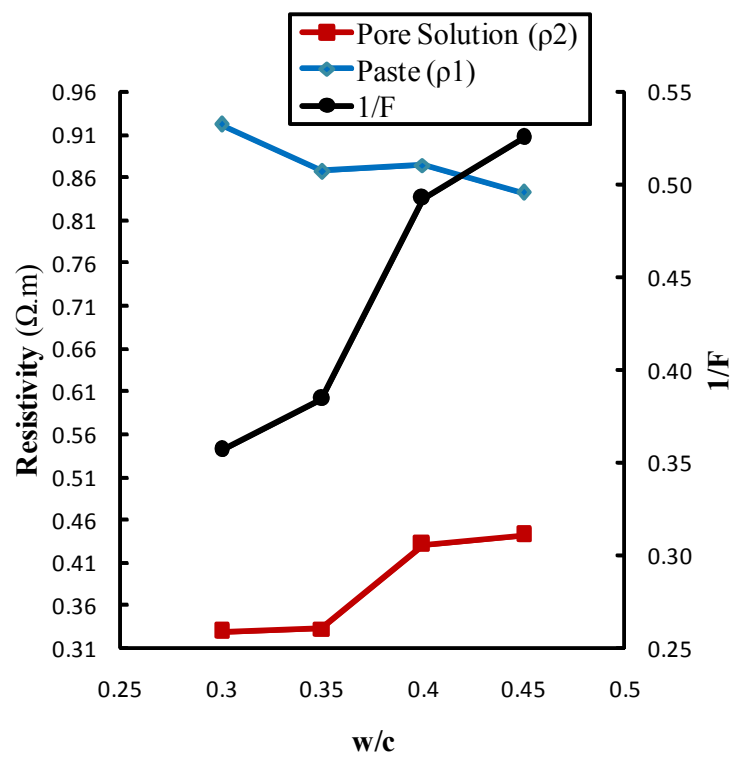

(h)

Figure B.1 (Continued). Paste and pore solution resistivity as well as inverse of formation factor versus $w / c$ at $30^{\text {th }}$ minute of paste age: a) OPC plus $0.2 \%$ superplasticizer; b) OPC plus $1.0 \%$ superplasticizer; c) OPC plus $10 \%$ fly ash; d) OPC plus $50 \%$ fly ash; e) OPC plus $5 \%$ silica fume; f) OPC plus 15\% silica fume; g) OPC plus 10\% slag; h) OPC plus $50 \%$ slag 


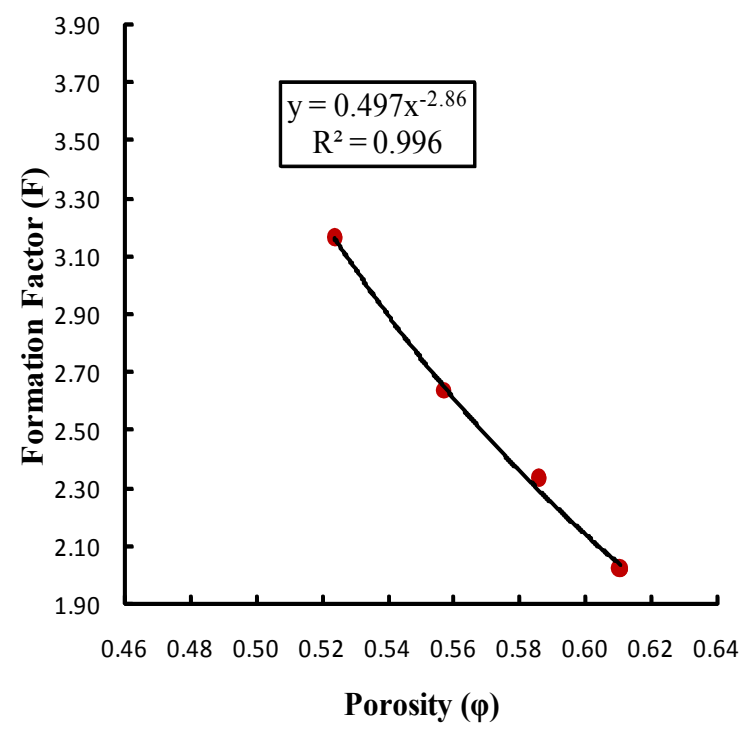

(a)

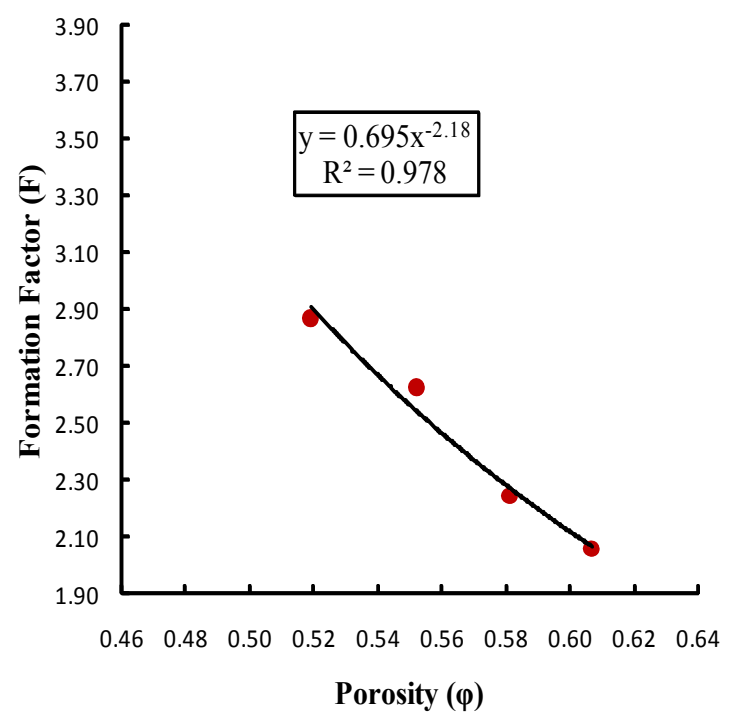

(c)

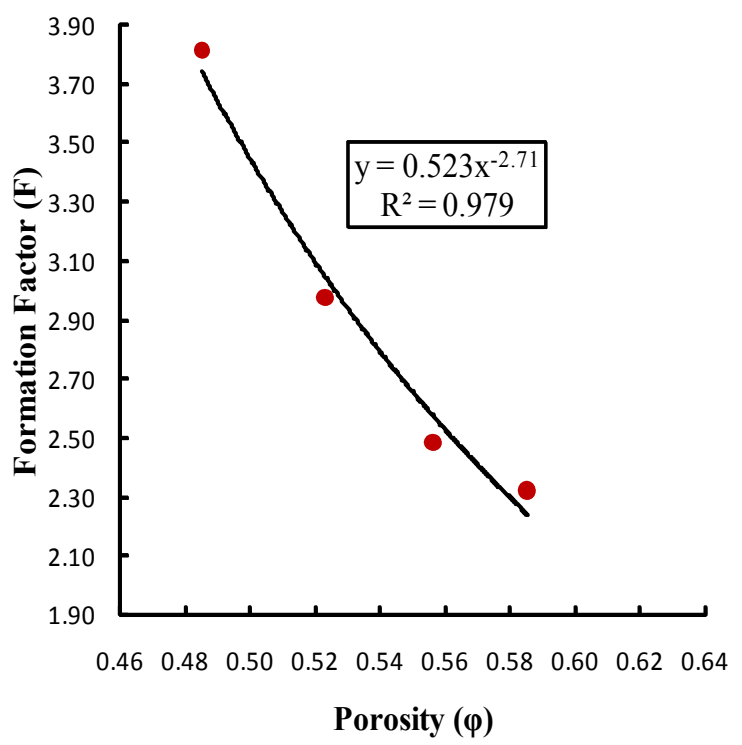

(b)

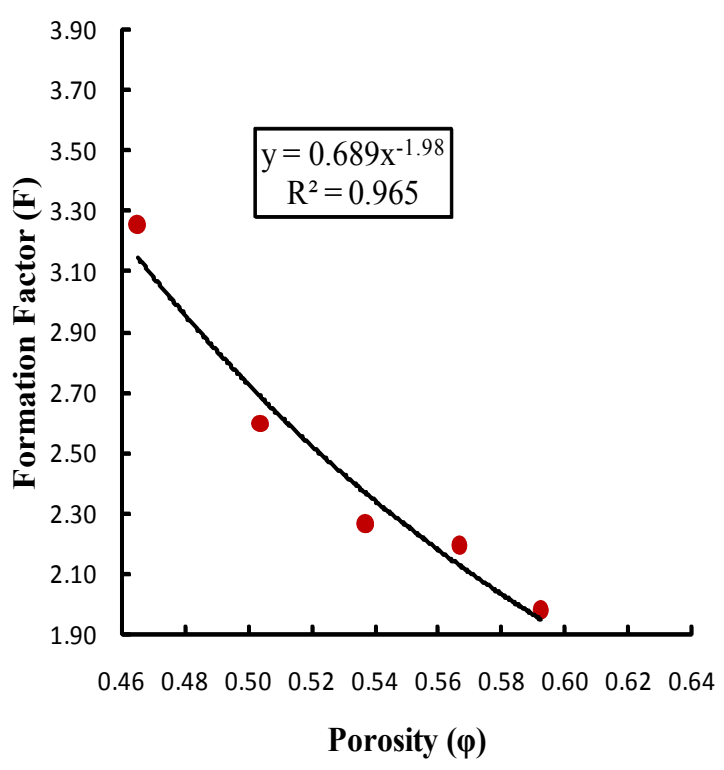

(d)

Figure B.2. Formation factor versus porosity of fresh cement pastes at $30^{\text {th }}$ minute of paste age: a) OPC plus $0.2 \%$ superplasticizer; b) OPC plus $1.0 \%$ superplasticizer; c) OPC plus $10 \%$ fly ash; d) OPC plus 50\% fly ash; e) OPC plus 5\% silica fume; f) OPC plus $15 \%$ silica fume; g) OPC plus 10\% slag; h) OPC plus 50\% slag 


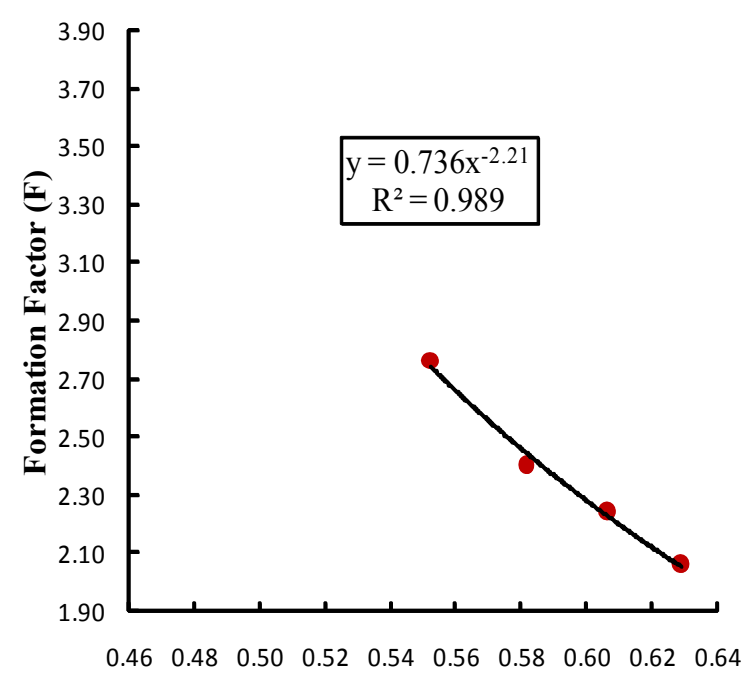

Porosity $(\varphi)$

(e)

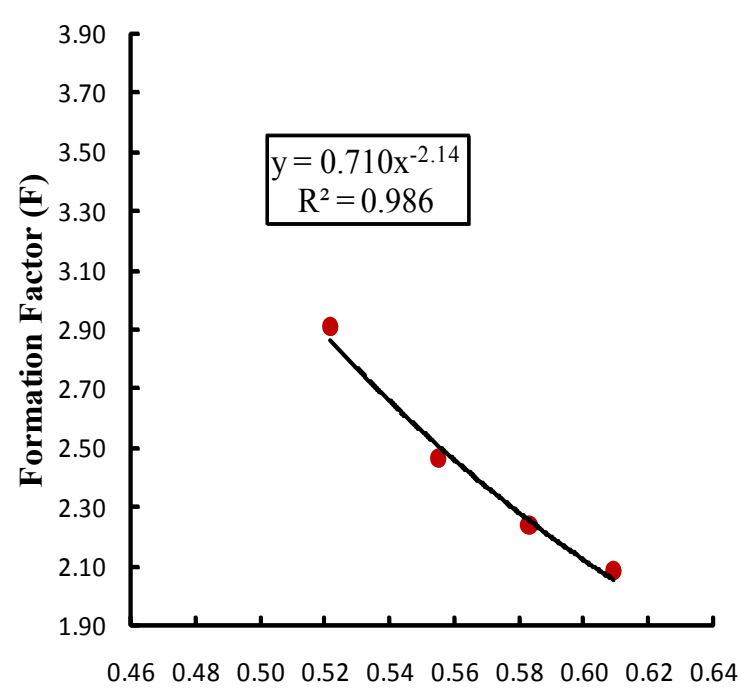

Porosity $(\varphi)$

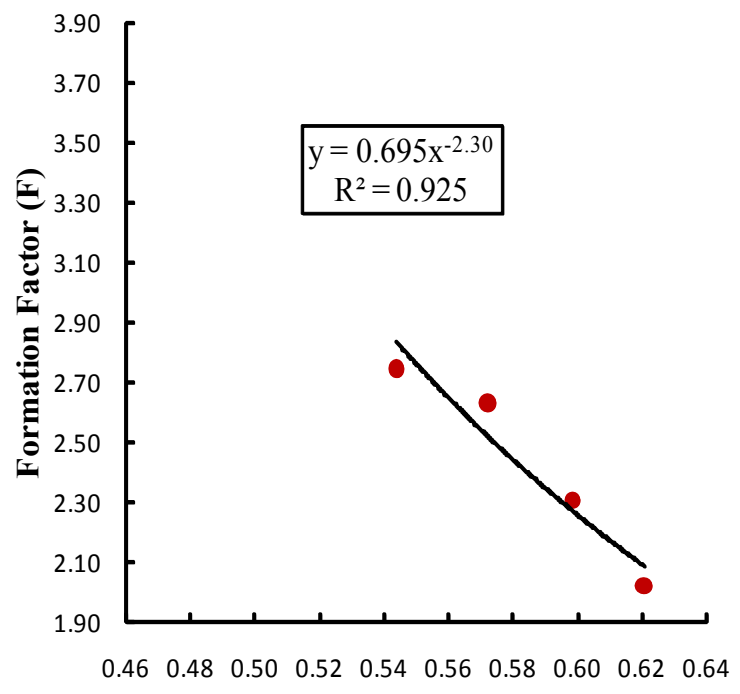

Porosity $(\varphi)$

(f)

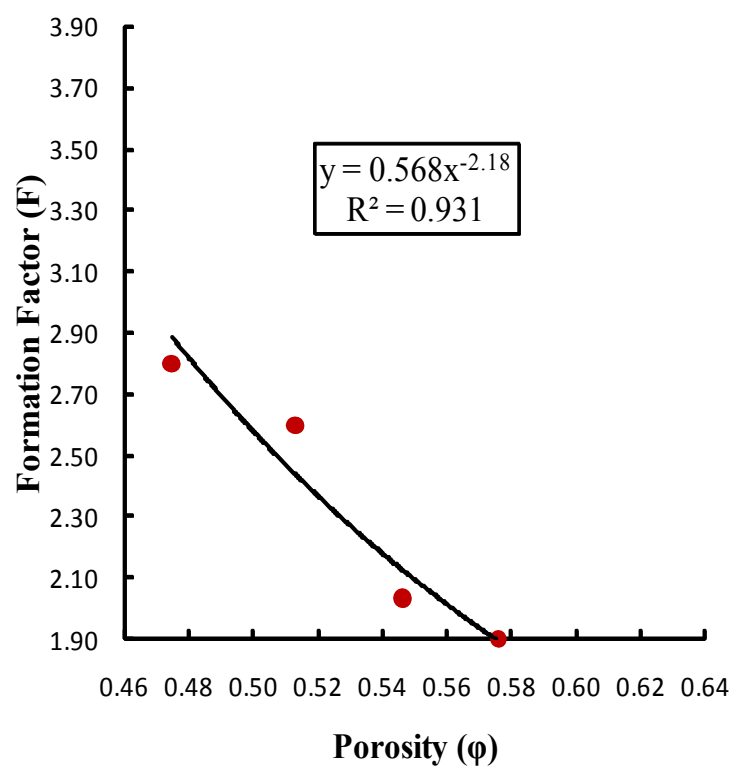

(h)

Figure B.2 (Continued). Formation factor versus porosity of fresh cement pastes at $30^{\text {th }}$ minute of paste age: a) OPC plus $0.2 \%$ superplasticizer; b) OPC plus $1.0 \%$ superplasticizer; c) OPC plus 10\% fly ash; d) OPC plus 50\% fly ash; e) OPC plus 5\% silica fume; f) OPC plus 15\% silica fume; g) OPC plus 10\% slag; h) OPC plus 50\% slag 


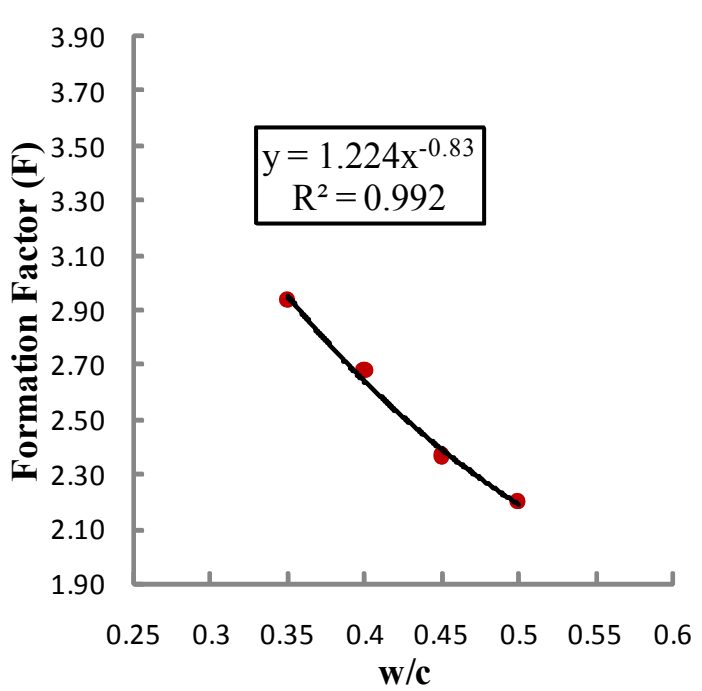

(a)

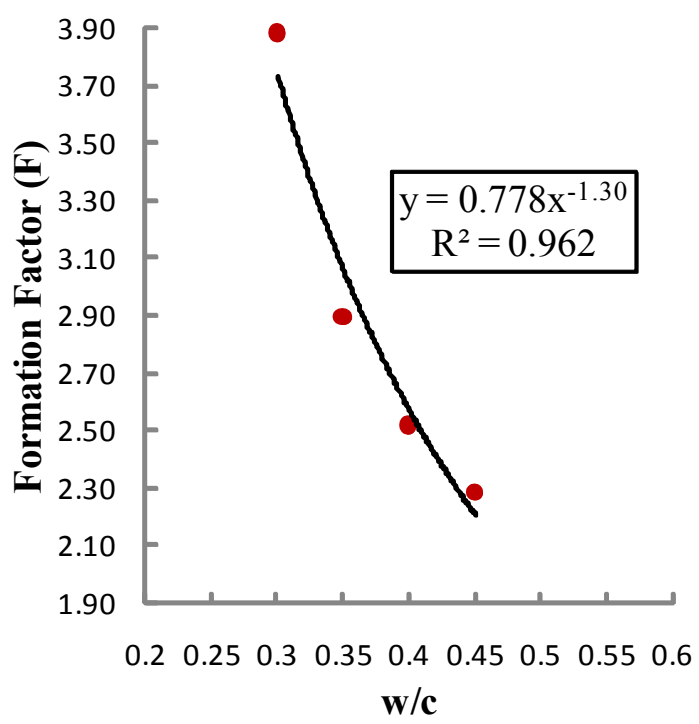

(c)

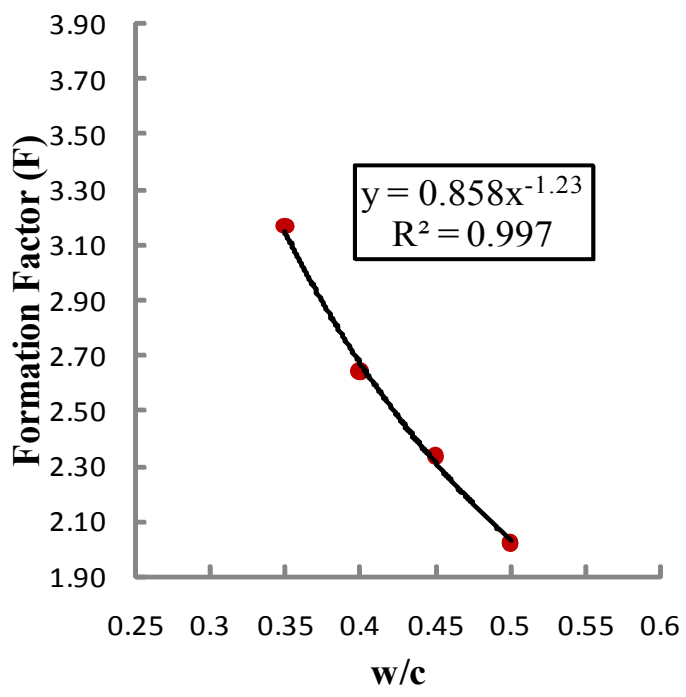

(b)

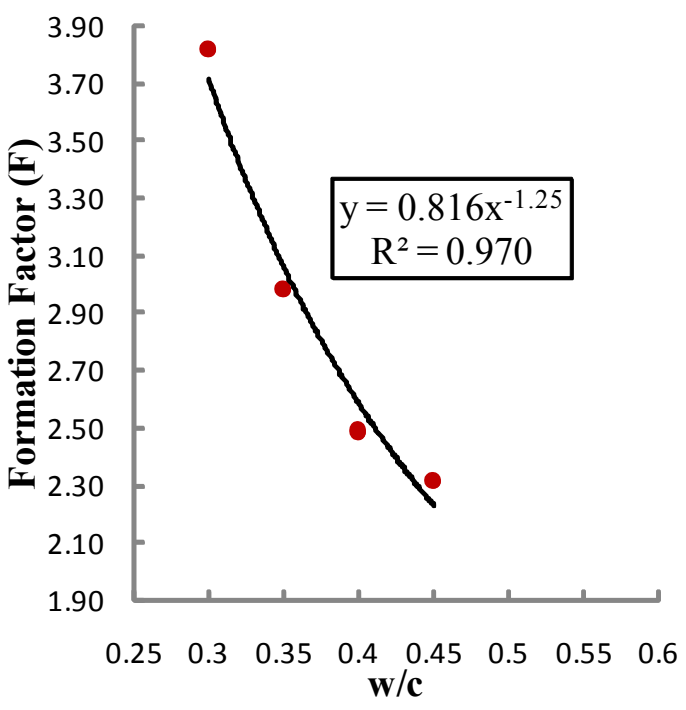

(d)

Figure B.3. Formation factor versus $\mathrm{w} / \mathrm{c}$ ratio of fresh cement pastes at 30th minute of paste age: a) OPC; b) OPC plus $0.2 \%$ superplasticizer; c) OPC plus $0.5 \%$ superplasticizer; d) OPC plus $1.0 \%$ superplasticizer ; e) OPC plus $10 \%$ fly ash; f) OPC plus $30 \%$ fly ash ; g) OPC plus 50\% fly ash; h) OPC plus 5\% silica fume; i) OPC plus $10 \%$ silica fume ; j) OPC plus 15\% silica fume; k) OPC plus $10 \%$ slag; 1) OPC plus $30 \%$ slag; m) OPC plus 50\% slag 


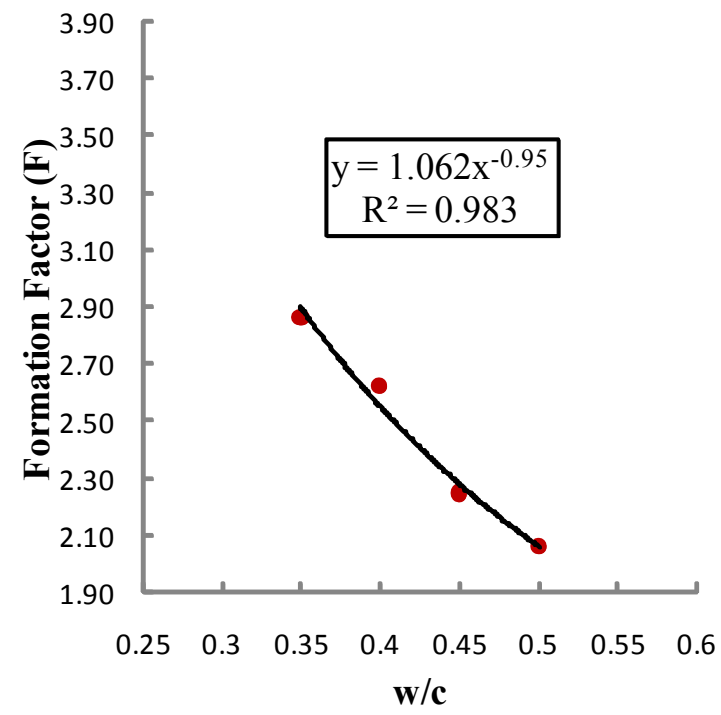

(e)

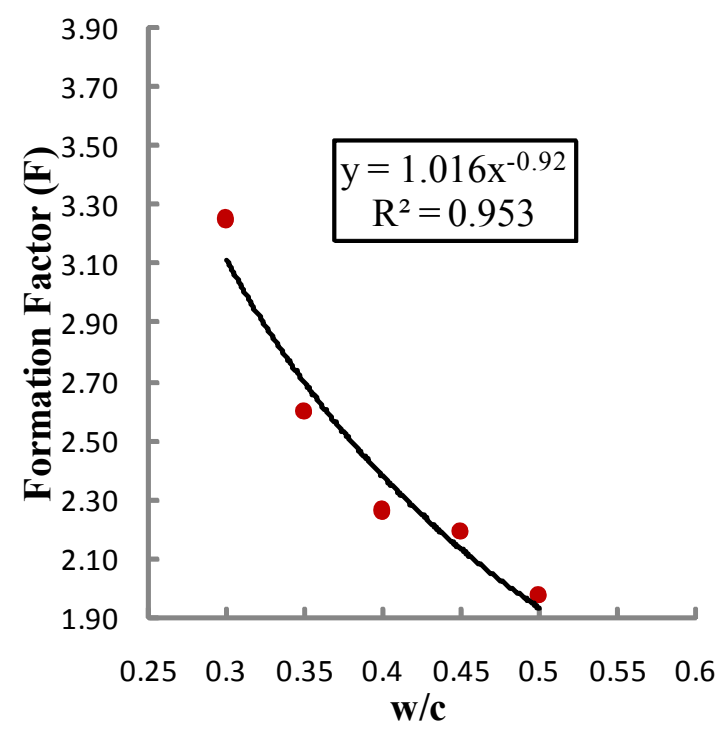

(g)

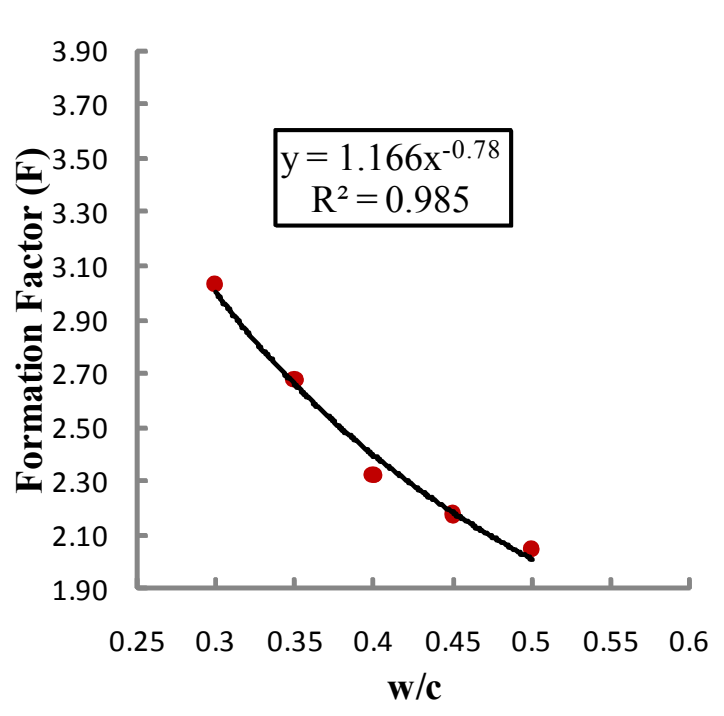

(f)

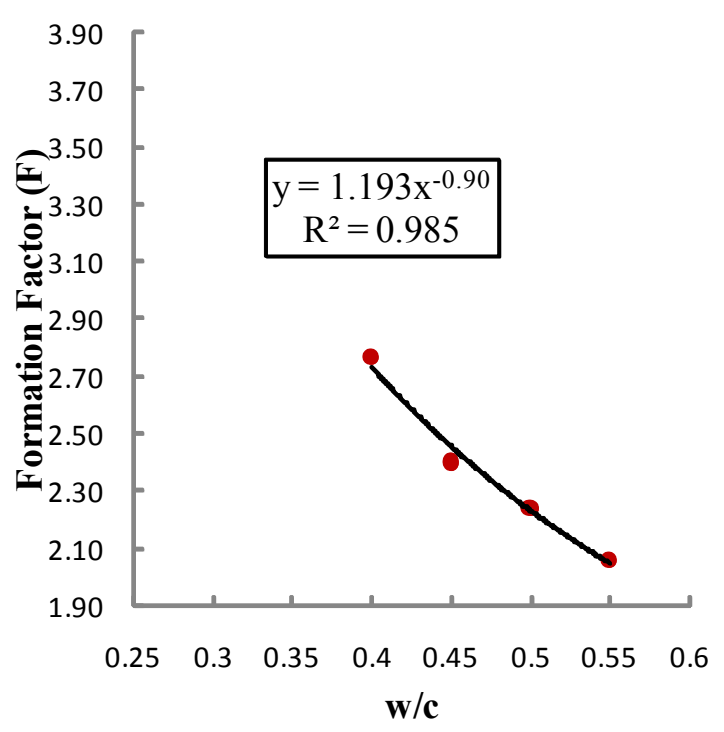

(h)

Figure B.3 (Continued). Formation factor versus w/c ratio of fresh cement pastes at $30^{\text {th }}$ minute of paste age: a) OPC; b) OPC plus $0.2 \%$ superplasticizer; c) OPC plus $0.5 \%$ superplasticizer; d) OPC plus 1.0\% superplasticizer ; e) OPC plus $10 \%$ fly ash; f) OPC plus $30 \%$ fly ash ; g) OPC plus 50\% fly ash; h) OPC plus 5\% silica fume; i) OPC plus $10 \%$ silica fume ; j) OPC plus $15 \%$ silica fume; k) OPC plus $10 \%$ slag; 1) OPC plus $30 \%$ slag; m) OPC plus 50\% slag 


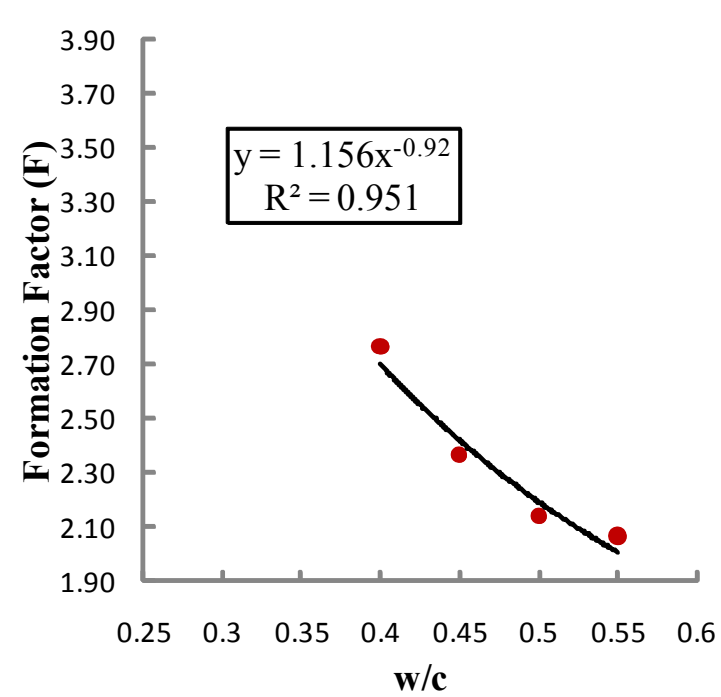

(i)

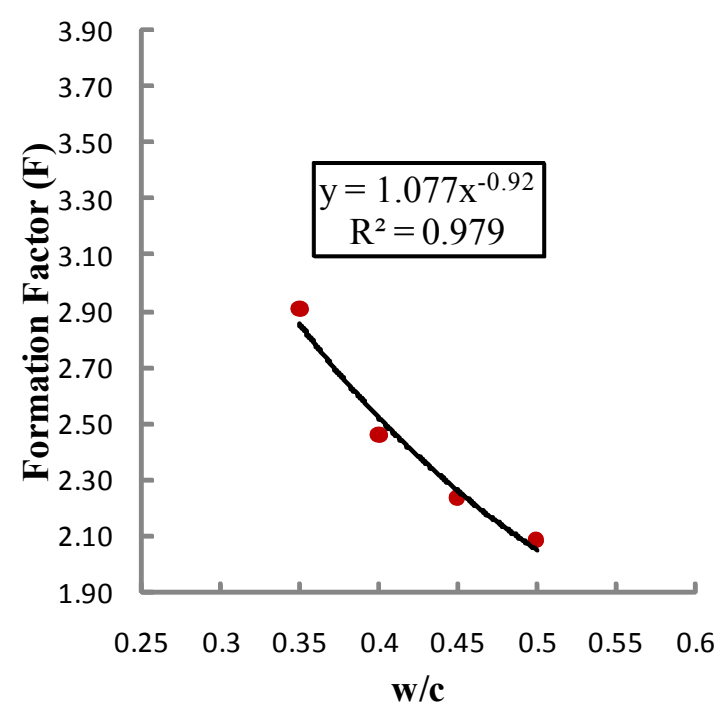

(k)

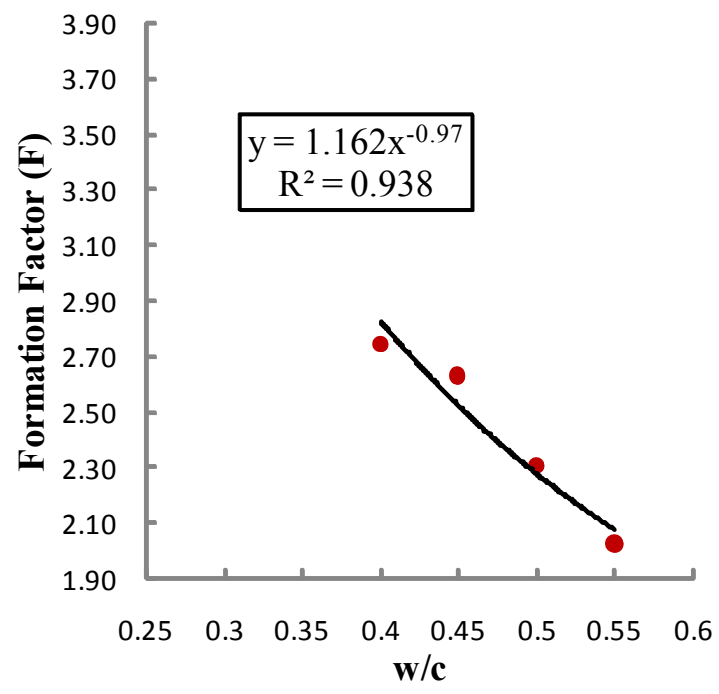

(j)

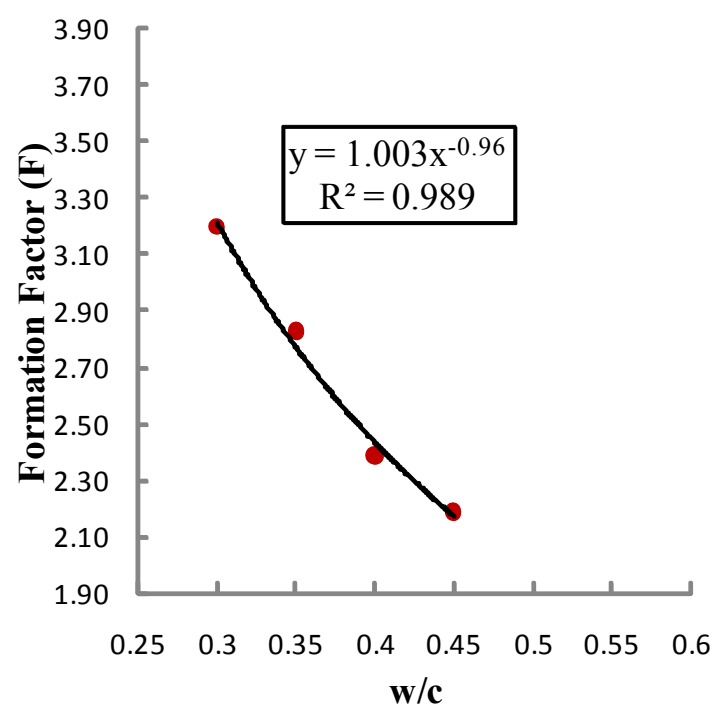

(l)

Figure B.3 (Continued). Formation factor versus w/c ratio of fresh cement pastes at $30^{\text {th }}$ minute of paste age: a) OPC; b) OPC plus $0.2 \%$ superplasticizer; c) OPC plus $0.5 \%$ superplasticizer; d) OPC plus 1.0\% superplasticizer ; e) OPC plus $10 \%$ fly ash; f) OPC plus $30 \%$ fly ash ; g) OPC plus $50 \%$ fly ash; h) OPC plus $5 \%$ silica fume; i) OPC plus $10 \%$ silica fume ; j) OPC plus $15 \%$ silica fume; $\mathrm{k}$ ) OPC plus $10 \%$ slag; 1) OPC plus $30 \%$ slag; m) OPC plus $50 \%$ slag 


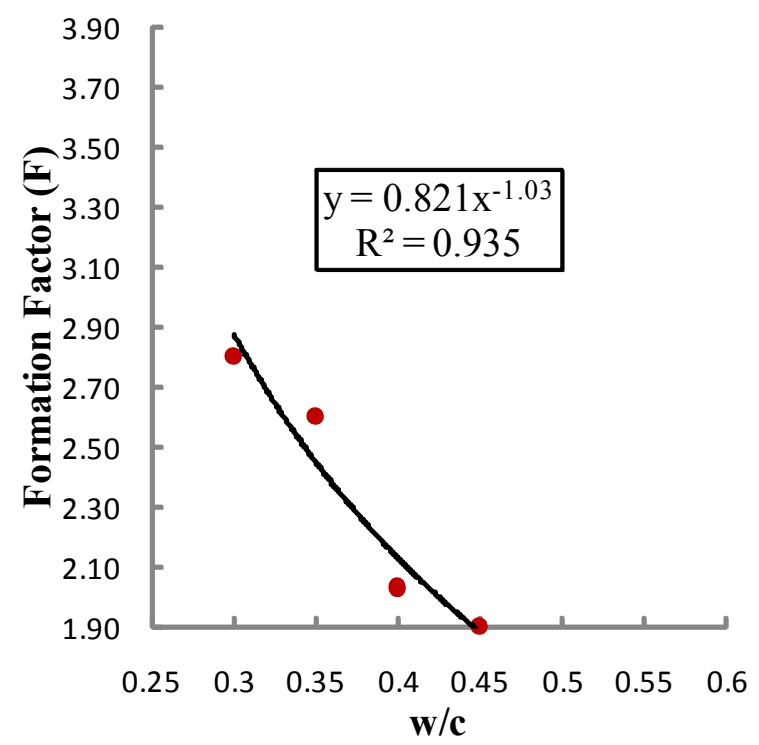

(m)

Figure B.3 (Continued). Formation factor versus $w / c$ ratio of fresh cement pastes at $30^{\text {th }}$ minute of paste age: a) OPC; b) OPC plus $0.2 \%$ superplasticizer; c) OPC plus $0.5 \%$ superplasticizer; d) OPC plus 1.0\% superplasticizer ; e) OPC plus $10 \%$ fly ash; f) OPC plus $30 \%$ fly ash ; g) OPC plus 50\% fly ash; h) OPC plus 5\% silica fume; i) OPC plus $10 \%$ silica fume ; j) OPC plus 15\% silica fume; k) OPC plus $10 \%$ slag; 1) OPC plus $30 \%$ slag; m) OPC plus 50\% slag 